\title{
Dinickel Complexes of the "Two-In-One" Pincer Scaffold.
}

\author{
Dissertation \\ Zur Erlangung des mathematisch-naturwissenschaftlichen \\ Doktorgrades \\ “Georg-August-Universität Göttingen” \\ Im Promotionsprogramm der Georg-August University School of \\ Science (GAUSS)
}

\author{
Vorgelegt von \\ Pierre Goursot \\ aus Paris
}

Göttingen 2019 


\section{Betreuungsausschuss}

Prof. Dr. Franc Meyer, Institut für Anorganische Chemie, Universität Göttingen

Prof. Dr. Inke Siewert, Institut für Anorganische Chemie, Universität Göttingen

\section{Referent}

Prof. Dr. Franc Meyer, Institut für Anorganische Chemie, Universität Göttingen

\section{Korreferent:}

Prof. Dr. Inke Siewert, Institut für Anorganische Chemie, Universität Göttingen

\section{Weitere Mitglieder der Prüfungskommission:}

Prof. Dr. Sven Schneider, Institut für Anorganische Chemie, Universität Göttingen

Prof. Dr. Ricardo Mata, Institut für Physikalische Chemie, Universität Göttingen

Dr. Michael John, Institut für Organische und Biomolekulare Chemie, Universität Göttingen

Dr. Hansmann Institut für Organische und Biomolekulare Chemie, Universität Göttingen

Tag der Mündliche Prüfung: 29.05.2019 


\section{Table of Content}

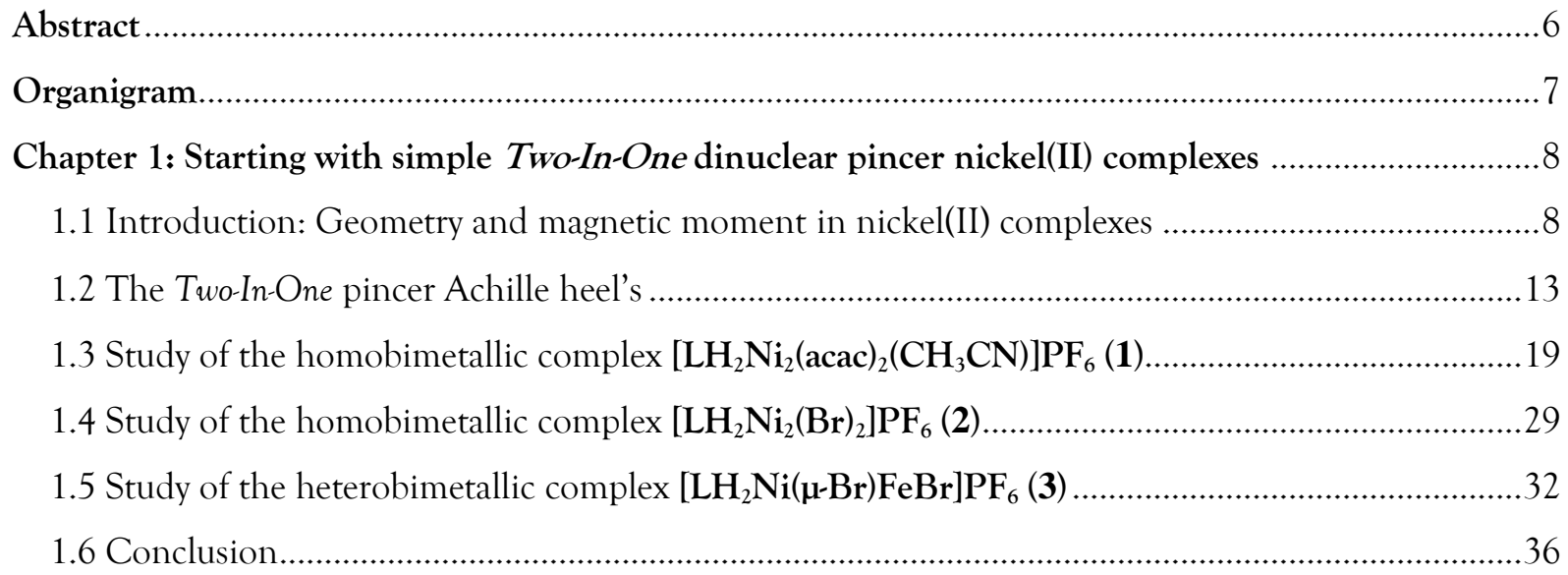

Chapter 2: Deprotonation and dearomatization of the ligand scaffold ...............................................37

2.1 Introduction: (De)aromatization: state of the art …........................................................................37

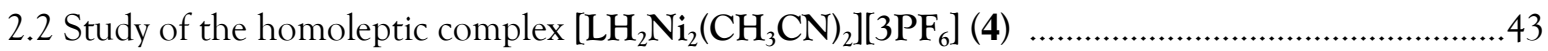

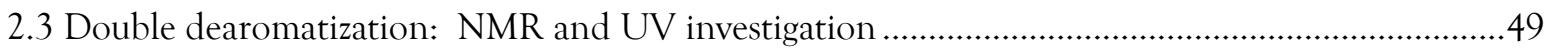

2.4 Observation of other dearomatized nickel(II) Two-In-One pincer complexes ...................................52

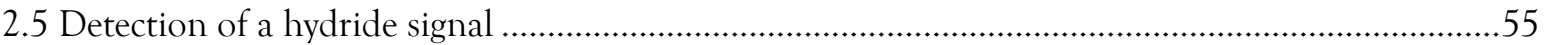

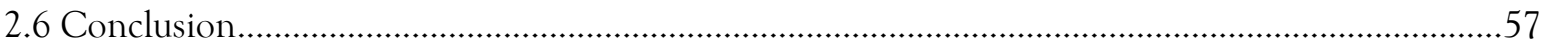

Chapter 3: A doubly dearomatized dihydride dinickel complex and the elusive cooperation of the

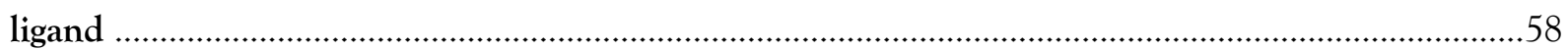

3.1 Introduction: Biorelevance of the nickel hydride motif for the anthropogenic and non-

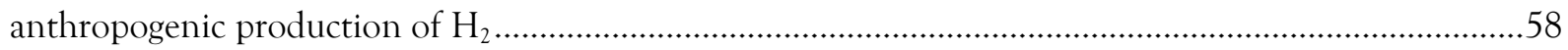

3.2 Isolation of a doubly dearomatized dihydride dinickel complex $[\mathrm{K}(2,2,2)]\left[\mathrm{LNi}_{2}(\mathrm{H})_{2}\right](7) \ldots \ldots \ldots \ldots 6$

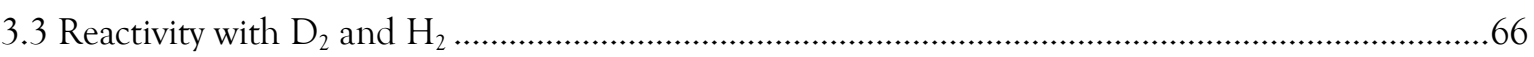

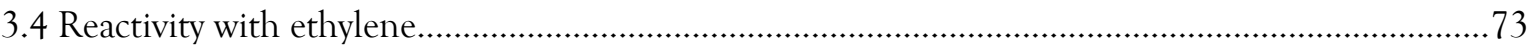

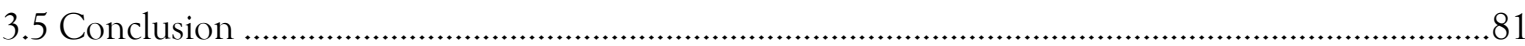

Chapter 4: Ligand cooperation and sequestration of an intramolecular dihydrogen bond ..................82

4.1 Introduction: The dihydrogen bond (DHB) in organometallic chemistry......................................82

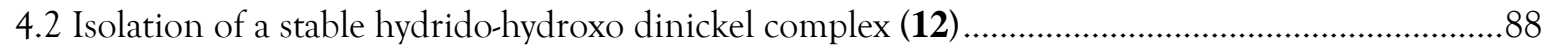

4.3 Characterization of an intramolecular dihydrogen bond (DHB) ……..........................................92

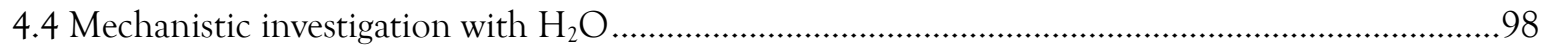

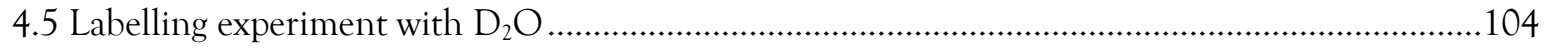




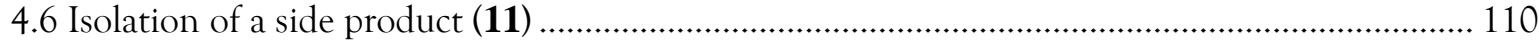

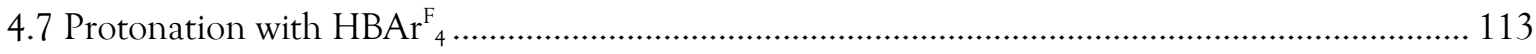

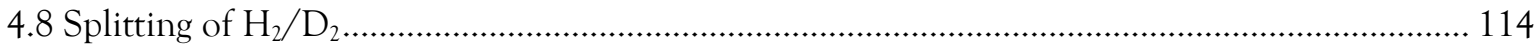

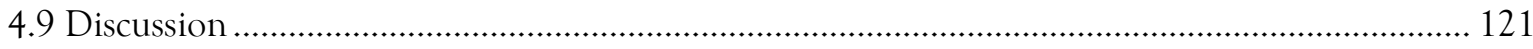

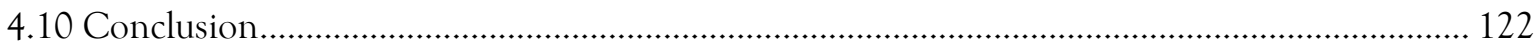

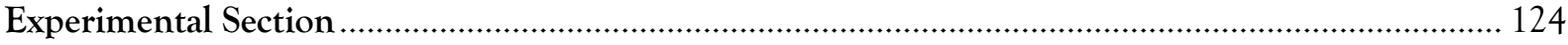

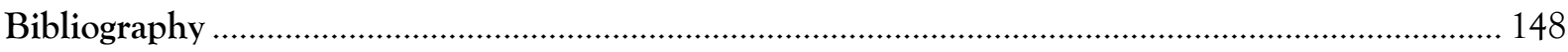

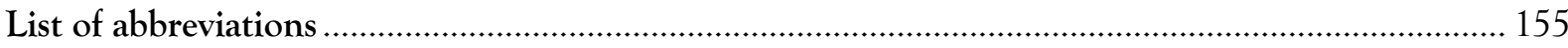

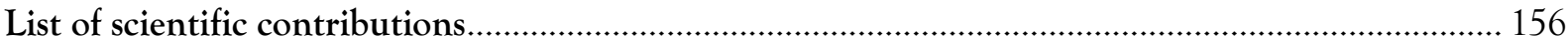




\begin{abstract}
:
Bringing two metal ions in close proximity offers interesting perspectives for cooperative activation and catalytic transformation of substrates. Ditopic pyrazolate-based bridging ligands are known to represent valuable ligand scaffolds in this regard. ${ }^{1}$ These studies on bimetallic systems featuring metal-metal cooperativity (MMC) are often inspired by multimetallic sites found in the active centers of metalloenzymes. ${ }^{2}$
\end{abstract}

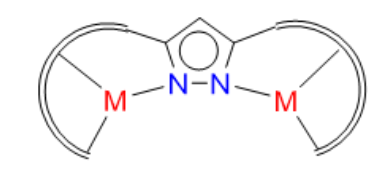

Metal-metal cooperativity (MMC)

Metal-ligand cooperation (MLC) is a ubiquitous concept applied for the activation and transformation of organic substrates in catalysis. ${ }^{3}$ The (de)aromatization of organometallic systems exemplified by Milstein's Ru catalyst, is a convenient method to perform polar bond dissociation during a catalytic event. ${ }^{97}$

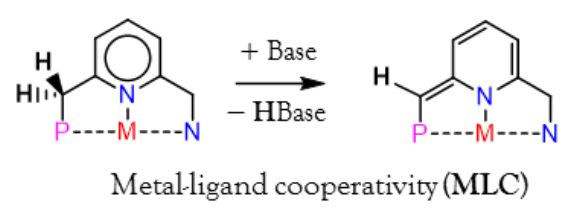

The implementation of such a ligand motif in a bimetallic scaffold is an approach to combine both intramolecular MMC and MLC. The following work continued the investigation made by S. Samanta in 2015, in which a "Two-In-One" di-iron complex was synthesized. ${ }^{114}$ This work extended the development of similar organometallic complexes by exploring the chemistry of nickel.

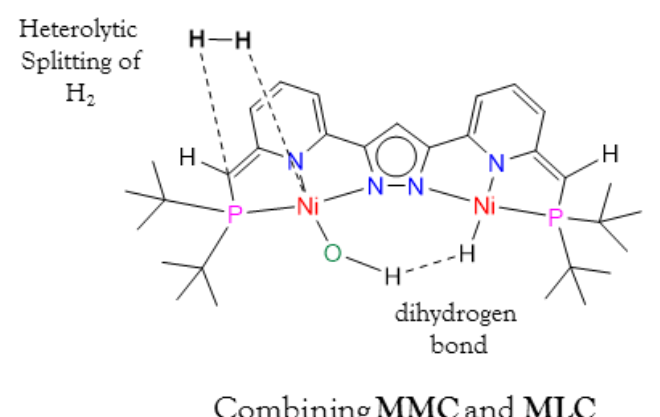

A major achievement of this work involves the introduction of a series of new dinickel complexes which were only different from each other by subtle structural modifications. Increased reactivities were observed by stepwise chemical modifications of the system. A highly reactive dinickel complex could be isolated. It displayed attractive reactivities toward the reversible cleavage of $\mathrm{H}_{2}$ by the possible cooperation of the ligand. This reactivity operated via a transverse intramolecular interaction, namely the dihydrogen bond. This subtype of hydrogen bond was stabilized by MMC. This work was an attempt to demonstrate the implication of both MMC and MLC during the chemical reaction of this new dinickel system. 


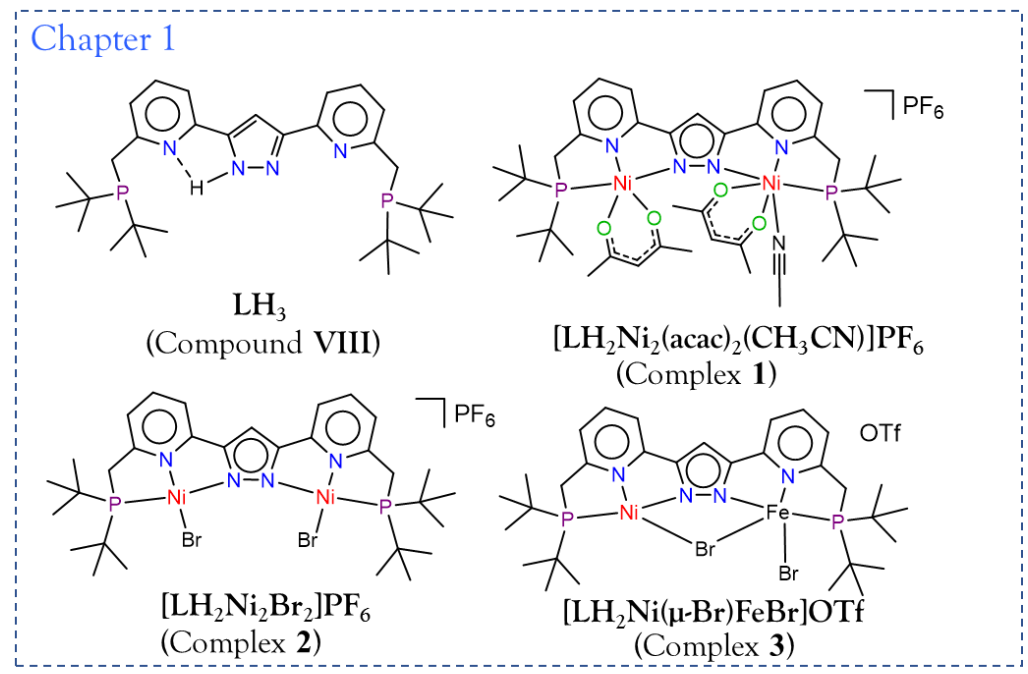

\section{Chapter 2}

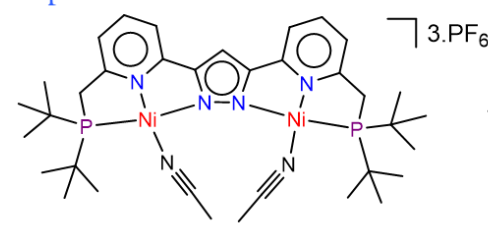

$\left[\mathrm{LH}_{2} \mathrm{Ni}_{2}\left(\mathrm{CH}_{3} \mathrm{CN}\right)_{2}\right]\left[\mathrm{PF}_{6}\right]$

(Complex 4)

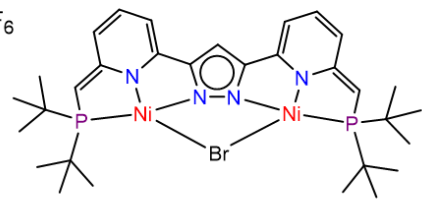

$\left[\mathrm{LNi}_{2}(\mu-\mathrm{Br})\right]$

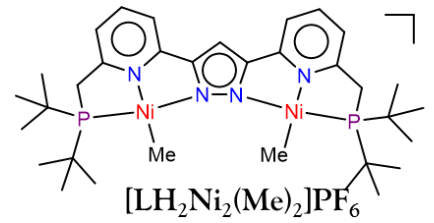

$\left[\mathrm{LH}_{2} \mathrm{Ni}_{2}\left(\mathrm{Me}_{2}\right]\right.$
(Complex $\mathbf{6})$

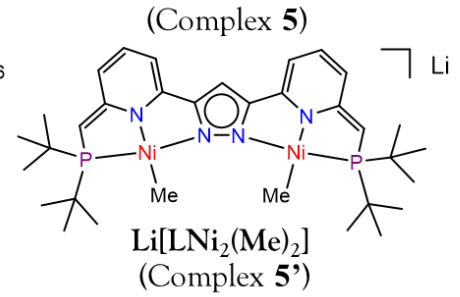

Chapter 3
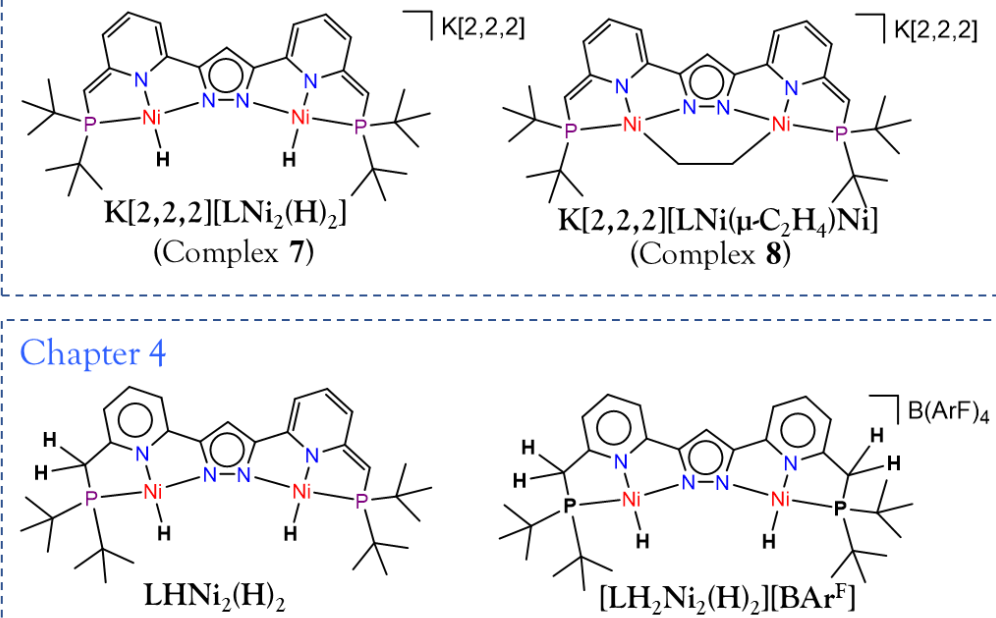

(Complex 9)
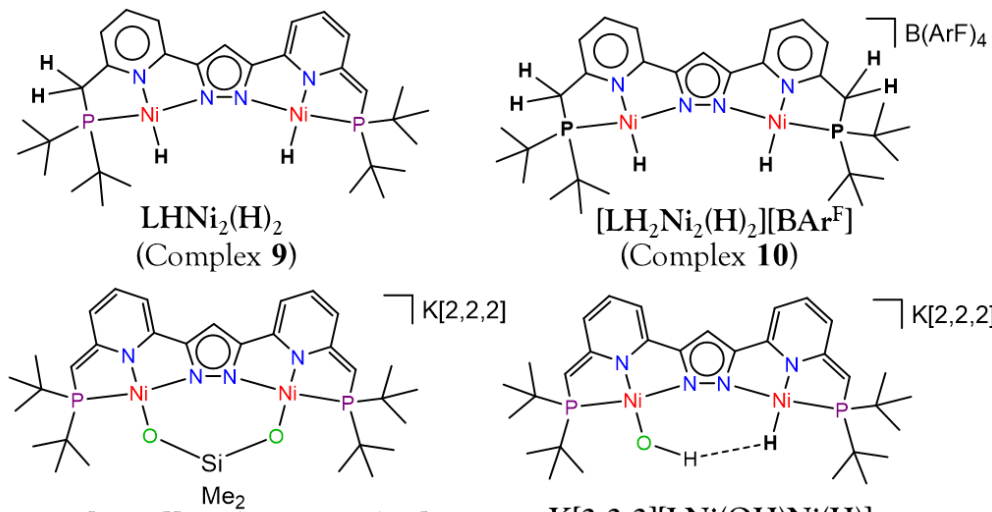

$\mathrm{K}[2,2,2]\left[\mathrm{LNi}\left(\mu-\mathrm{O}_{2} \mathrm{SiMe}_{2}\right) \mathrm{Ni}\right]$ (Complex 11)

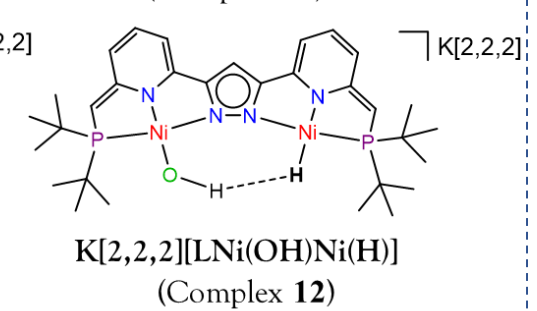

\section{Organigram}




\section{Chapter 1: Starting with simple Two-In-One dinuclear pincer Nickel(II) complexes.}

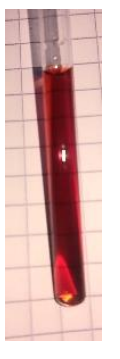

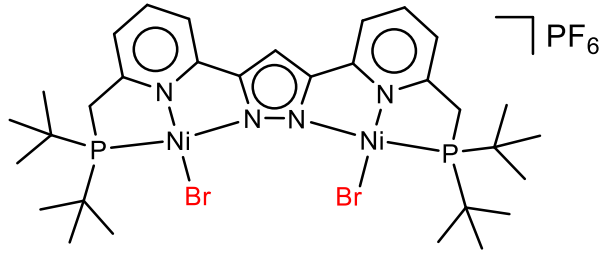

Diamagnetic red $\mathrm{S}=0$

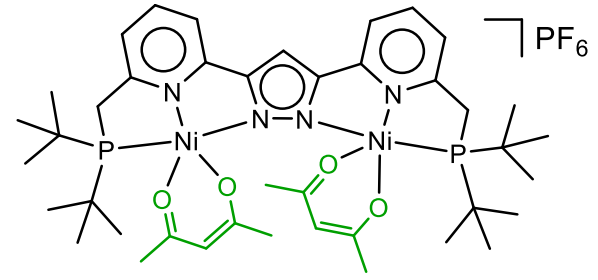

Paramagnetic green $\mathrm{S}=2$

High-spin or low-spin for two-in-one $\mathrm{Ni}^{2+}$ complexes

In organometallic chemistry, nickel ions displayed reactivities which were not always easy to tame. ${ }^{4}$ Nickel was a metal that already found numerous practical applications in catalysis since the beginning of the $20^{\text {th }}$ century and was extensively used by P. Sabatier and J. B. Senderens for the catalytic hydrogenation of organic substrates. ${ }^{5}$ The catalyst was called "nickel de Sabatier" but its exact molecular identity was not defined. Sabatier became famous for the so called "SabatierSenderens reduction" and won the Nobel prize in $1912 .{ }^{6}$ At the time, nickel was compared to a "spirited horse" and this qualification has been explained recently. ${ }^{7}$ Later, a special effect due to the presence of nickel ("nickel effect") was found during the polymerization of olefins. ${ }^{8}$ The extension of catalytic reactions involving $\mathrm{Ni}$ in homogenous catalysis led to their classification into different categories (C-C coupling, C-H activation, etc...), which were recently reviewed. ${ }^{9}$

A particular attention regarding the reactivity, the geometry, the spin state and their interconnectivity is required for the mechanistic understanding of chemical processes. For example, $\mathrm{Ni}$ ions in the oxidation state of $+\mathrm{II}$ are possibly high-spin (paramagnetic) or low-spin (diamagnetic). The coordination of a substrate or solvent molecule could trigger a change in the magnetic properties. The following introduction will explore the changes of spin states of $\mathrm{Ni}^{2+}$ complexes. The understanding of their magnetic properties in solution is a prerequisite for the next chapters.

\subsection{Geometry and magnetic moment in nickel(II) complexes.}

Since the beginning of pincer chemistry in the 70's by the introduction of tridentate chelating systems, ${ }^{10}$ nickel became even more attractive for the synthesis of new complexes, as it was cheap, very reactive and abundant on earth. ${ }^{11}$ A first approach to get reactive complexes consisted of making unsaturated coordination platforms, so that the substrate could easily bind to the reactive site. In this regard, $\mathrm{d}^{8}$ square planar complexes were appreciated for their reactivity and diamagnetism. The latter property allowed utilization of NMR methods as a tool for a deeper investigation on intermediates involved in a catalytic cycle. For $\mathrm{d}^{8}$ metals, the geometry was often 
correlated to the spin state, however this relationship was not always unequivocal. ${ }^{12}$ For example, the complete characterization of $\mathrm{Ni}(\mathrm{acac})_{2}$ has been disputed in the scientific community before it came to a consensus with the publication of the X-ray structure. ${ }^{13}$ The molecular structure unravelled octahedral geometries around nickel atoms in paramagnetic trimers $\mathrm{Ni}_{3}(\mathrm{acac})_{6} \cdot{ }^{13}$ The ambiguous spin state of $\mathrm{Ni}^{2+}$ in solution was rationalized by the existence of an oligomerization equilibrium in solution between a square planar (diamagnetic $S=0$ ) and an oligomeric (paramagnetic $S=1$ ) form. ${ }^{14,15,16}$ This oligomerization process was influenced by the steric hindrance induced by the substituents on the ligand (Figure 1.1). ${ }^{17}$ Therefore, the coordination mode of the ligand in correlation with the spin state of the complex demanded attention. Today, the geometric changes in $\mathrm{M}(\mathrm{acac})_{\mathrm{n}}$ is still an interesting topic. ${ }^{18}$ Generally, the molecular geometries of $\mathrm{Ni}^{2+}$ complexes can be predicted based on their colours in the solid state: square planar $\mathrm{Ni}^{2+}$ complexes mostly have a colour ranging from violet to red and brown. Octahedral $\mathrm{Ni}^{2+}$ complexes generally fall between shade of green to blue.
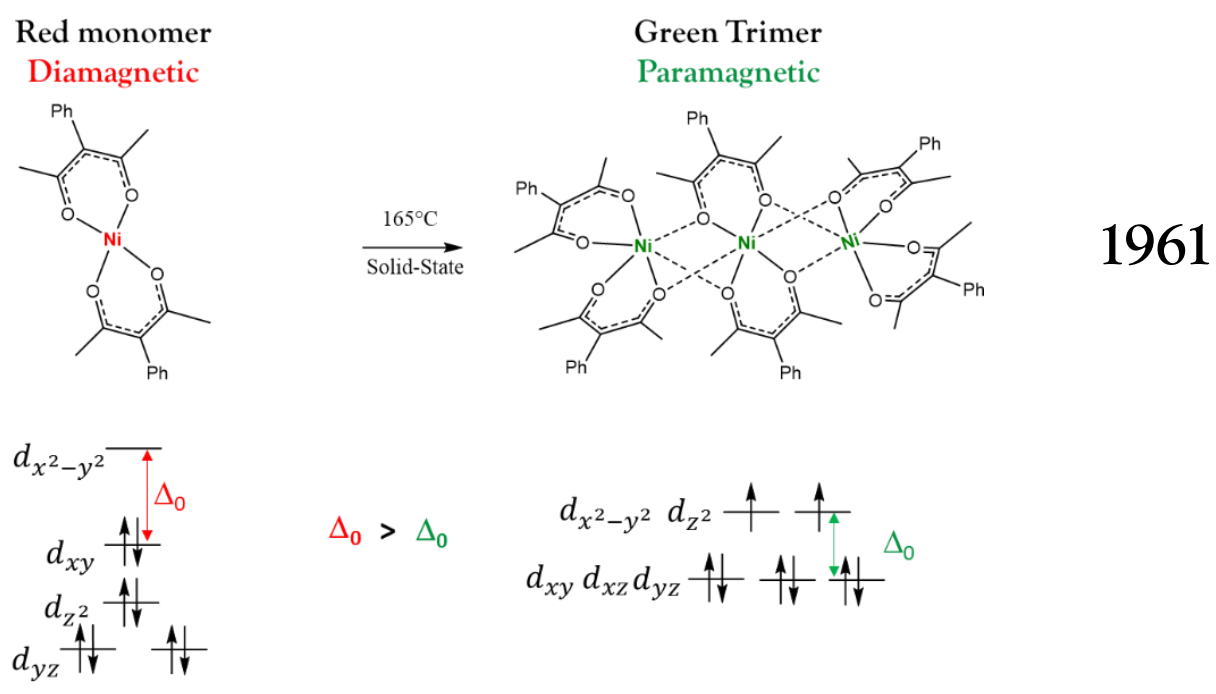

Figure 1.1. The thermal oligomerization of square planar to octahedral $\mathrm{Ni}^{2+}$ complexes in the solid state. ${ }^{14,15,16}$

The geometry adopted by $\mathrm{Ni}^{2+}$ complexes was early discussed in terms of steric and electronic factors induced by the ligands. ${ }^{19}$ For example, strongly donating phosphine ligands were having high ligand field strength and rather favoured tetracoordination $(n=4)$. Two geometries were possible for a complex which had a coordination number $n=4$ : square planar or tetrahedral. In some phosphine based $\mathrm{Ni}^{2+}$ complexes with $\mathrm{n}=4$, these two possible geometries were in equilibrium. A "type of geometrical isomerism" implicating the thermal distortion between these two forms was accompanied by a singlet-triplet spin state equilibrium and aroused great interest. ${ }^{20,21,22}$ Inspired by this kind of equilibrium, the development of photomagnetic $\mathrm{Ni}^{2+}$ switches has recently been explored. ${ }^{23}$ 
The use of more acidic phosphine ligands led to the formation of pentacoordinated $\mathrm{Ni}^{2+}$ complexes $(n=5) \cdot{ }^{24,25}$ Multidentate ligands with strongly donating P atoms, later called "pincer" systems, were also involved in pentacoordinated $\mathrm{Ni}^{2+}$ complexes. Some examples of low-spin pentacoordinated $\mathrm{Ni}^{2+}$ complexes ${ }^{26}$ were represented in Figure 1.2. An interesting feature was the N-H moiety of complexes $\mathbf{A}^{37} \mathbf{B}^{27}$ and $\mathbf{C}^{28}$, which possibly interacted with the apical bromide by hydrogen bonding. It could have affected the flexibility of the $\mathrm{Ni}-\mathrm{Br}$ bond and the spin state of the complex according to processes which will be discussed below.

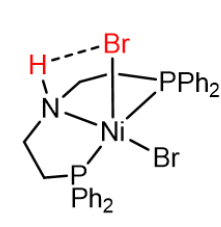

1970

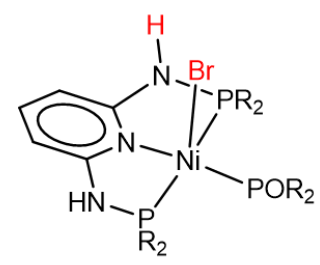

2006

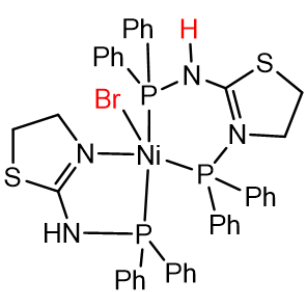

2010<smiles></smiles>

2016

A

B

C

Figure 1.2. Diamagnetic pentacoordinated $\mathrm{Ni}^{2+}$ complexes. ${ }^{26,27,28,37}$

Pentacoordinated $\mathrm{Ni}^{2+}$ complexes were reviewed and could be high-spin or low-spin. ${ }^{29}$ Low spin pentacoordinated $\mathrm{Ni}^{2+}$ complexes were particularly interesting, as they were supposed to be close from a magnetic crossover point. ${ }^{30}$ More precisely, square pyramidal complexes $(n=5)$ could be regarded as square planar complexes $(n=4)$ with an additional ligand in the apical position of the basal plane. In such a complex, the plasticity of the apical ligand was critical for the determination of the spin state of the complex. ${ }^{31} \mathrm{~A}$ shorter bond length between the metal and the apical ligand likely favoured a triplet state, while a longer bond length favoured the singlet state. ${ }^{32,33}$ Examples of spin transition in pentacoordinated $\mathrm{Ni}^{2+}$ complexes were represented in Figure $1.3^{34,35}$ and Figure 1.4. ${ }^{36}$ In both cases, a temperature change likely triggered the lengthening between the metal and the apical atom (the $\mathrm{Cl}^{-}$in one case, the nitrogen of a pyrazole in the other), so that the paramagnetic complex became diamagnetic. The coordination number of the metal $(n=5)$ was preserved during the spin transition. In Figure 1.4, the high-spin complex had a "strong" square pyramidal character. At high temperature, an axial bond elongation and an equatorial bond contraction were observed. The resulting low-spin complex was closer from the square planar geometry and was qualified as weak square pyramidal. 


\section{Apical Elongation}

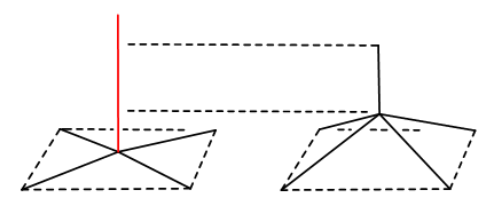

Tetracoordination Pentacoordination

Diamagnetic Paramagnetic

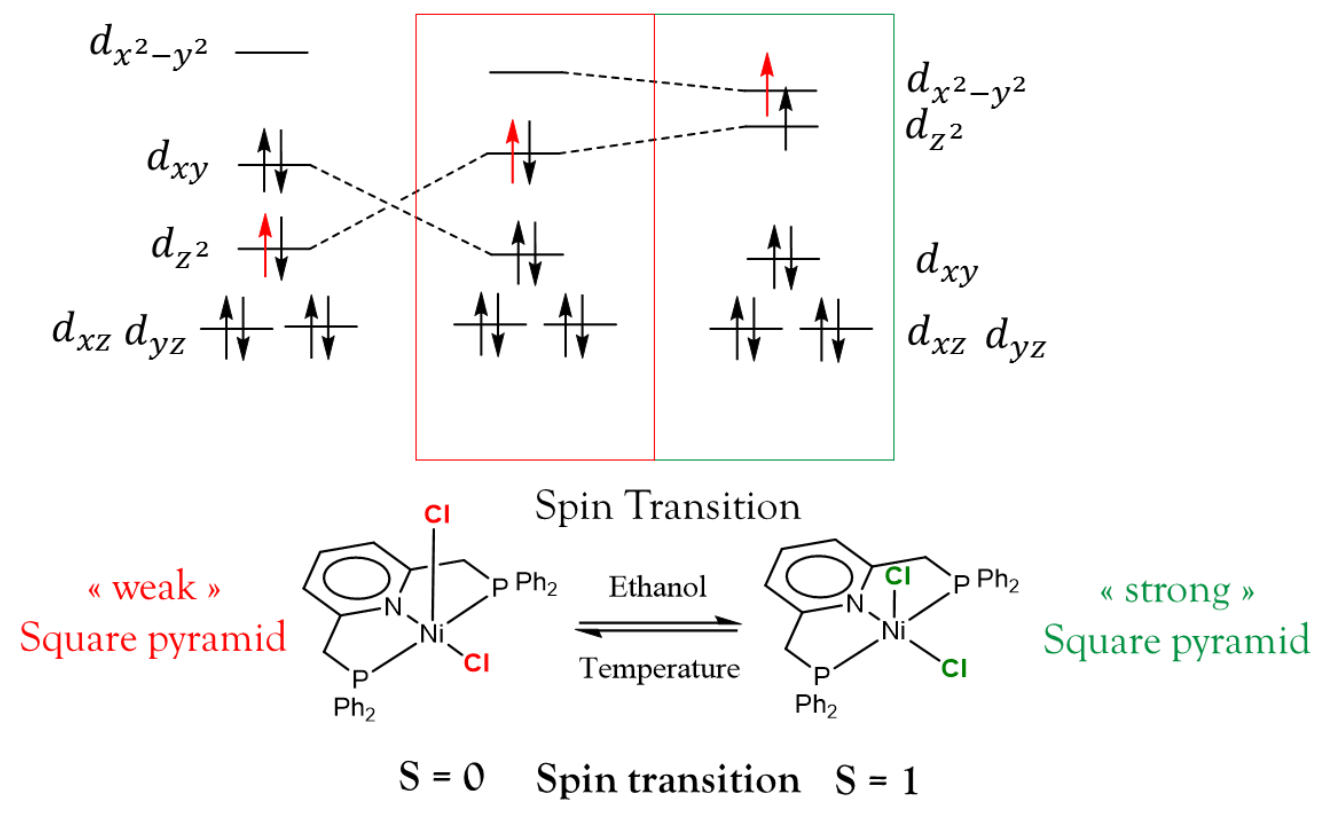

Figure 1.3. Orbital splitting diagram for the spin transition in a pentacoordinated $\mathrm{Ni}^{2+}$ complex. ${ }^{33,34,35}$

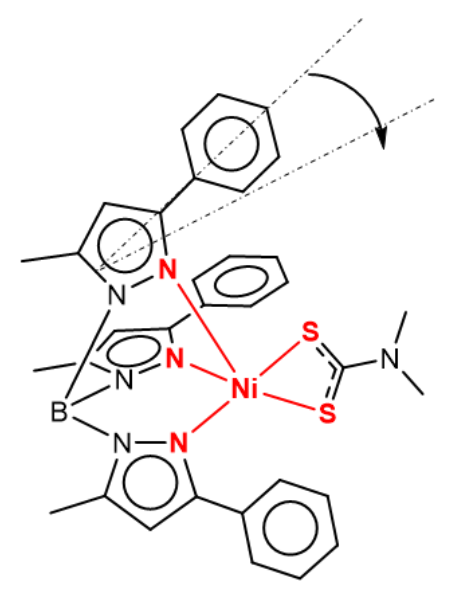

"weak "

Square pyramid

Diamagnetic

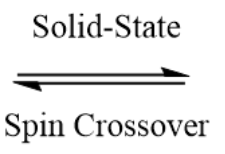

Spin Crossover

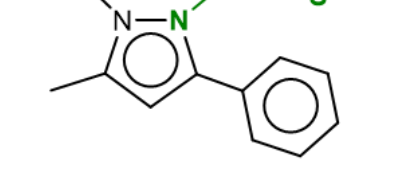

"strong"

Square pyramid

Paramagnetic

Figure 1.4. Spin crossover in a pentacoordinated $\mathrm{Ni}^{2+}$ complex. ${ }^{36}$ 
In contrast to the previous cases where the coordination numbers were constant during the spin transition, some $\mathrm{Ni}^{2+}$ complexes were able to undergo a spin transition by the coordination of an external ligand. The coordination of a ligand to a low-spin square planar complex $(n=4)$ gave rise to a high-spin pentacoordinated complex $(n=5){ }^{37,77}$ If the ligand was in excess, it possibly led to high-spin octahedral complex. ${ }^{38}$ This phenomenon had recently gained more attention and was named CISSS (coordination induced spin-state switching). ${ }^{39}$ Square planar $\mathrm{Ni}^{2+}$ complexes were valuable scaffolds for the study of CISSS as they often displayed low-spin state. Indeed, paramagnetic square planar $\mathrm{Ni}^{2+}$ complexes are extremely rare. ${ }^{40}$ In this regard, porphyrins were appreciable chelating macrocycles that allowed the sequestration of the $\mathrm{Ni}^{2+}$ ion in the square planar geometry for the study of CISSS. . $^{41,42,43,44}$

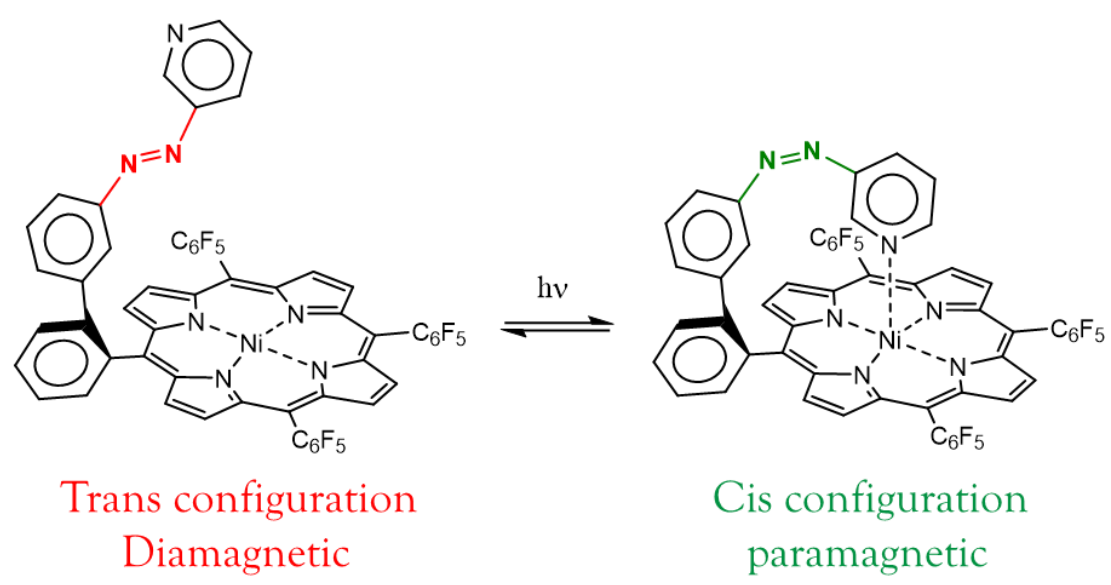

Figure 1.5. Light induced spin transition in $\mathrm{Ni}^{2+}$ complexes with a net geometrical change of the metal center. ${ }^{43}$

To sum up the occurrence of spin transitions in $\mathrm{Ni}^{2+}$ complexes, three cases can be mentioned: a) when the coordination number $\mathrm{n}=4$ within the complex is constant during the spin transition: a distortion between square planar to tetrahedral geometry is involved. $\mathrm{b}$ ) when the coordination number $\mathrm{n}=5$ is constant during the spin transition: the apical ligand of a square pyramidal complex undergoes an elongation, which changes the spin state of the complex. c) when the coordination number of the complex varies from $n=4$ (mostly low spin) to $n=5,6$ (mostly highspin). This phenomenon is called CISSS (coordination induced spin state switching). 


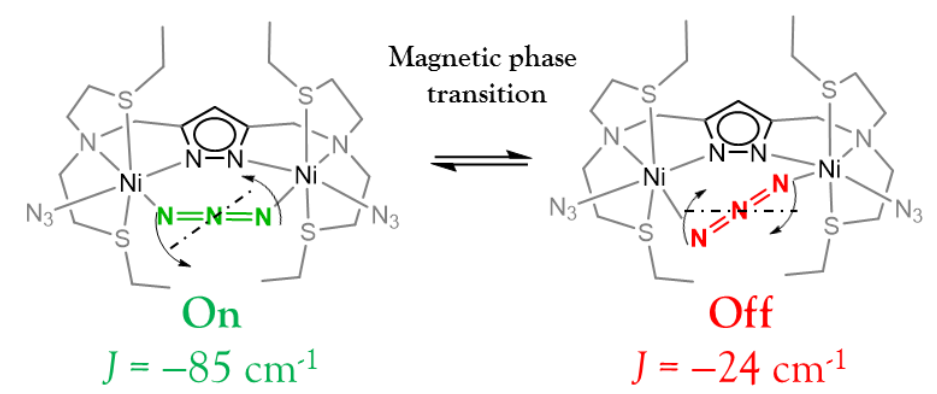

Figure 1.6. Expanding the complexity of magnetic properties in dinuclear $\mathrm{Ni}$ complexes involving pyrazolate scaffold. Spin transition was not occurring, but a temperature dependence of the magnetic exchange coupling value $J$ was responsible for the unexpected magnetic bistability. ${ }^{45}$

Since this doctoral contribution involved pyrazolate based dinickel complexes, an interesting case of magnetic bistability was considered. In 2005, a complex involving two octahedral $\mathrm{Ni}^{2+}$ ions bridged by a $\mathrm{N}_{3}{ }^{-}$azido unit was shown to undergo a structural modification in the solid state as depicted in Figure 1.6..$^{45}$ Varying the temperature led to a tilting of the azido bridge, which induced changes in $J$, the coupling constant. Strong antiferromagnetic coupling was observed at low temperature. Increasing the temperature led to smaller $|\mathrm{J}|$ values. Other multidentate pyrazolate ligands suitable for the synthesis of dinickel complexes had been synthesized in order to explore the potential of cooperative effects between the two metals. Their magnetic properties $^{46,47}$ and their reactivity for olefin polymerization ${ }^{48,49}$ was studied.

In this chapter, dinickel complexes involving the ligand VIII will be developed. A green paramagnetic complex $\left[\mathrm{LH}_{2} \mathrm{Ni}_{2}(\mathrm{acac})_{2}\left(\mathrm{CH}_{3} \mathrm{CN}\right)\right] \mathrm{PF}_{6}(1)$ and a red coloured diamagnetic complex $\left[\mathrm{LH}_{2} \mathrm{Ni}_{2}\left(\mathrm{Br}_{2}\right] \mathrm{PF}_{6}(2)\right.$ will be compared. The synthesis of a heterobimetallic complex $\left[\mathrm{LH}_{2} \mathrm{Ni}(\mu-\right.$ $\mathrm{Br}) \mathrm{FeBr}] \mathrm{PF}_{6}(3)$ will be realized.

\subsection{The Two-In-One pincer Achille heel's.}

Compound VIII, the so called "Two-In-One pincer" (Figure 1.9), has been synthesized by S. Samanta in 2014. ${ }^{114}$ As described in the introduction, this ligand can be considered as two typical PNN pincer ligands of "Milstein's Catalyst" that are fused together by a pyrazole linker unit. It is synthesized in seven steps in a total yield of $4 \%$. Technical aspects of the synthesis such as improving the yields or exploring the scope of substituents that can be attached on the phosphorus atom have been revisited recently by A. Gers-Barlag in his dissertation (2016) and are still under investigation today. Even if the synthetic route is established, the present contribution will highlight some curiosities that have not been discussed previously. 


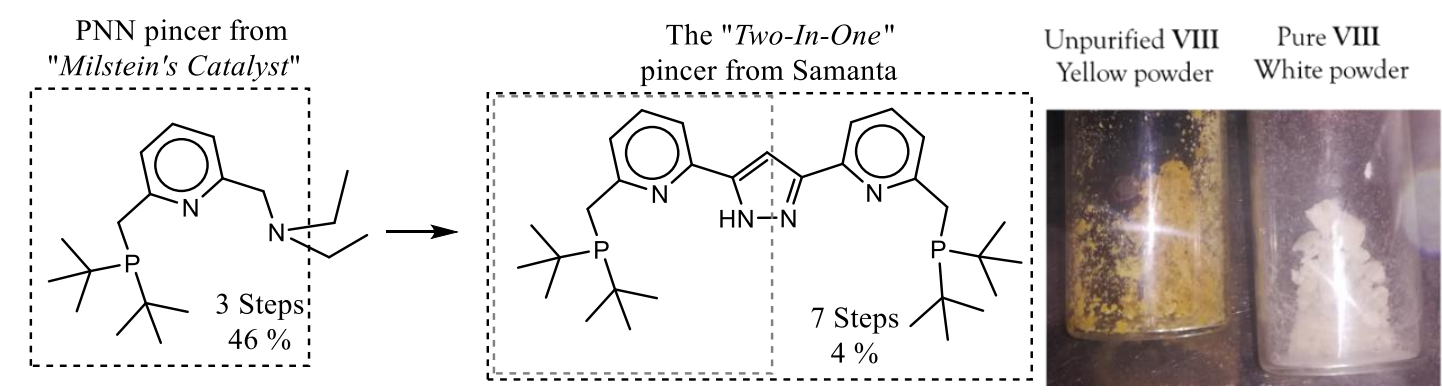

Figure 1.7. Representation and photography of the proligand VIII synthesised by Samanta, ${ }^{114}$ inspired by Milstein's system. ${ }^{78}$

The first reaction of the ligand synthesis consisted of a six electron chemical oxidation of 2,6Lutidine (I) to the corresponding carboxylic acid II by $\mathrm{KMnO}_{4}{ }^{214}$ It is a process that is mainly used today even if it has already been reported in $1949 .{ }^{214}$ Still after 70 years, one wouldn't draw mechanistic arrows of the addition of $\mathrm{MnO}_{4}^{-}$on a $-\mathrm{CH}_{3}$ group and the subsequent elimination of $\mathrm{MnO}_{2}$ without uncertainty. The "complexity of this simple" reaction was that a temperaturecontrolled addition of the oxidizing agent afforded the kinetic product (II) but the formation of the dicarboxylic acid (the 12 e oxidation of I), however, was unavoidable and lowered the yield of the reaction. The convenience of this reaction laid in the simple and efficient purification method that allowed II to be isolated as pure material. ${ }^{214}$

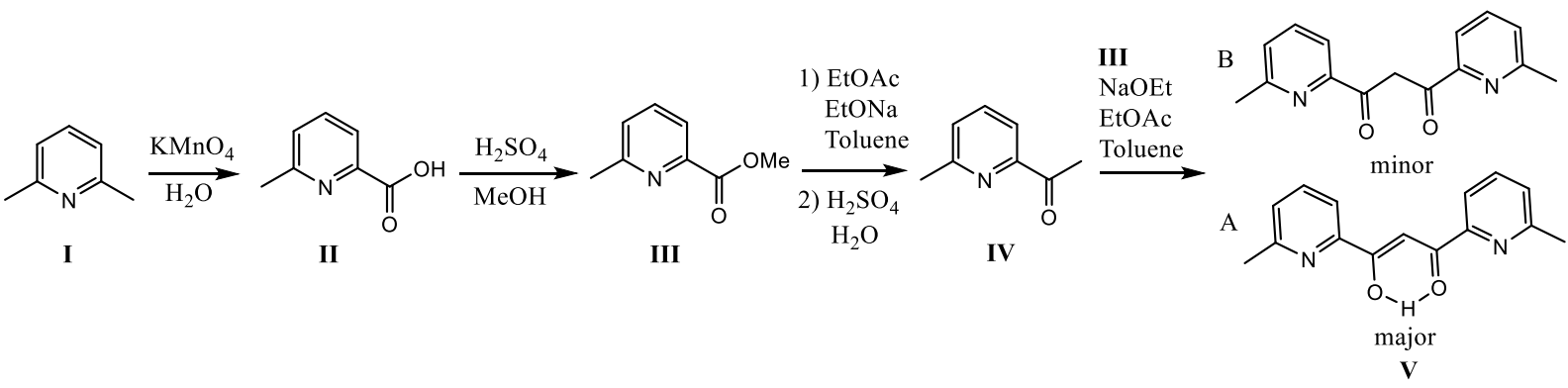

Figure 1.8. Synthetic pathway for the diketone $\mathrm{V}$

Compound II was converted to the methyl ester III, ${ }^{50}$ which was then reduced to the ketone IV by a classical Claisen condensation followed by a decarboxylation step. Another Claisen condensation of III with IV afforded the tautomer mixture $\mathrm{V} .{ }^{50}$ It was interesting to note that the generally desired diketone of the reaction was actually the minor tautomer of the mixture. Even if the NMR data reported in the literature mentioned the presence of the enol and the attention was focused on the diketone synthesis, ${ }^{50}$ it was found that the enol form of $\mathrm{V}$ was preferentially formed in a ratio of 81:19 under the reaction conditions. The proton of the alcohol function in $\mathrm{V}$, which was hydrogen bonded to the oxygen of the carbonyl, often hidden in the baseline, was detected at $16 \mathrm{ppm}$ in the ${ }^{1} \mathrm{H}$ NMR spectrum (Figure 1.10). Even if $\mathrm{V}$ was present as two tautomeric forms, compound VI was the major product obtained in the next reaction, indicating that keto-enol equilibrium in $\mathrm{V}$ likely occurred, as the reaction shifts to the formation of the product. 


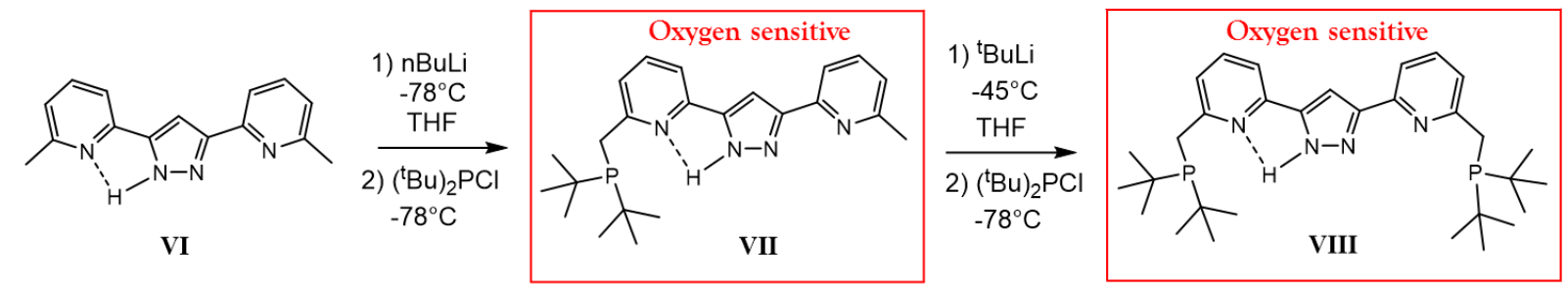

Figure 1.9. Synthetic pathway for the "Two-In-One" pincer VIII.

A Paal-Knorr reaction gave the aromatic pyrazole in compound VI by the traditional condensation of $\mathbf{V}$ with hydrazine. ${ }^{50}$ The proton bound to the nitrogen atom of the pyrazole was hydrogen bonded to the nitrogen of the pyridine and was detected as a broad signal at $9.0 \mathrm{ppm}$ in the ${ }^{1} \mathrm{H}$ NMR spectrum. Some of the protons on the pyridine moieties gave broad signals, which likely indicated a weak asymmetric character in complex VI (Figure 1.11).
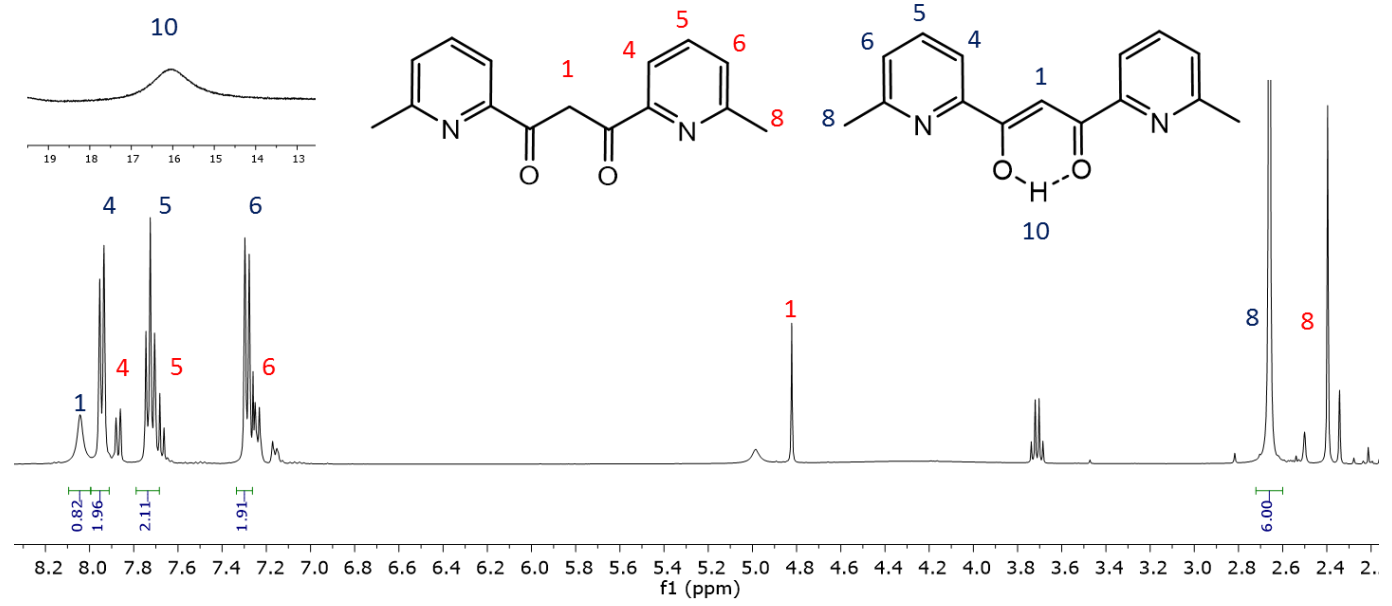

Figure 1.10. ${ }^{1} \mathrm{H}$ NMR $(400 \mathrm{MHz})$ spectrum of $\mathrm{V}$ in $\mathrm{CDCl}_{3}$. Two tautomeric forms are observed. 


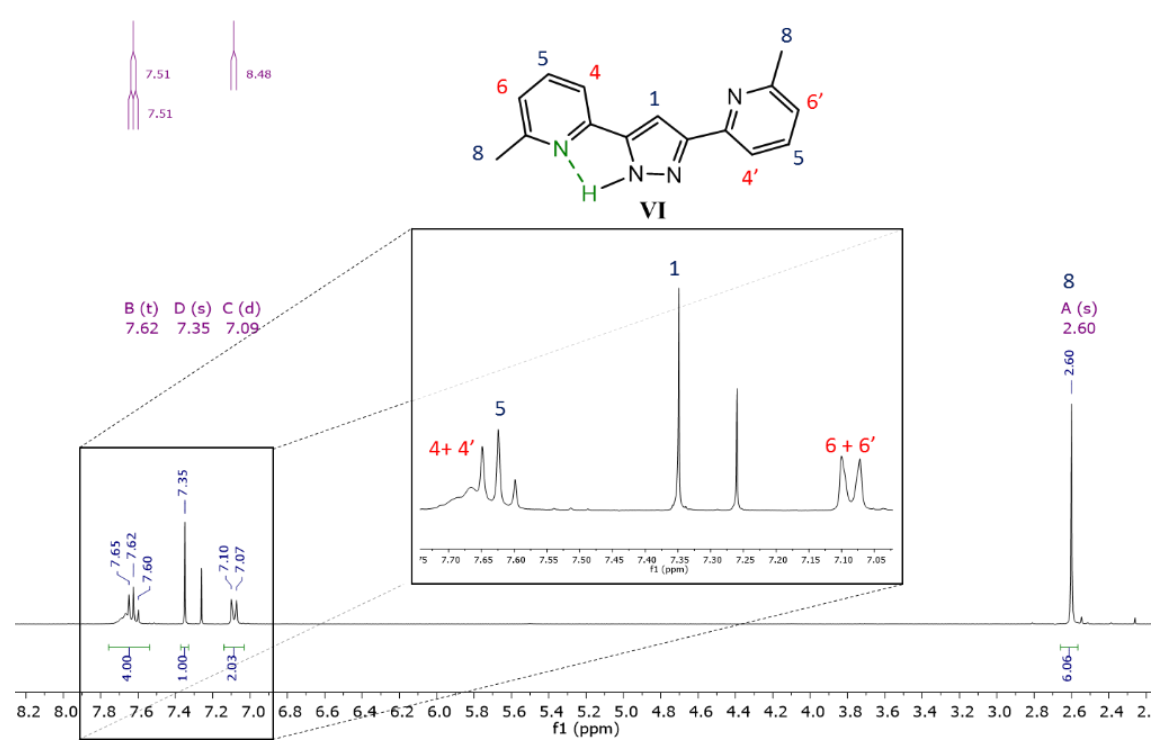

Figure 1.11. ${ }^{1} \mathrm{H}$ NMR $(300 \mathrm{MHz})$ spectrum of VI in $\mathrm{CDCl}_{3}$.

Compound VII was obtained after nucleophilic substitution on $(t \mathrm{Bu})_{2} \mathrm{PCl}$ by the dilithium salt of VI, obtained after treatment with 2.5 equivalents of $n \mathrm{BuLi}$. One equivalent of nBuLi reacted thoroughly with the proton of the pyrazole, and a second equivalent was needed for the more difficult deprotonation of the methyl group. An issue that was not solved was the low yield of the second phosphorylation step leading to VIII.

While VII was obtained in quantitative yield, VIII was only obtained with $22 \%$ yield. We observed that changing $n \mathrm{BuLi}$ to $\mathrm{tBuLi}$ slightly increased the yield but not significantly. There was no apparent reason why this reaction was not quantitative unless the potential acidity of the protons located on the phosphomethylene arm of VII was considered. Indeed, P. Braunstein has demonstrated by X-ray and NMR methods that organolithium bases deprotonated similar functional pincer systems and gave their corresponding lithium complexes (Figure 1.12), ${ }^{51} \mathrm{a}$ phenomenon that could possibly be transposed to the reaction of VII with tBuLi : Instead of deprotonating the $-\mathrm{CH}_{3}$ in compound VII, $t \mathrm{BuLi}$ possibly deprotonated the $-\mathrm{CH}_{2}$ group of the phosphomethylene arm (Figure 1.13) of which acidity was dependent on the substituent of the phosphorus atom. The acidity of the proton increased when the alkyl groups induced less electron donation: $\left\{(t \mathrm{Bu})_{2} \mathrm{P}-\mathrm{CH}_{2}<(i \mathrm{Pr})_{2} \mathrm{P}-\mathrm{CH}_{2}<(\mathrm{Ph})_{2} \mathrm{P}-\mathrm{CH}_{2}\right\}$ (Figure 1.13).
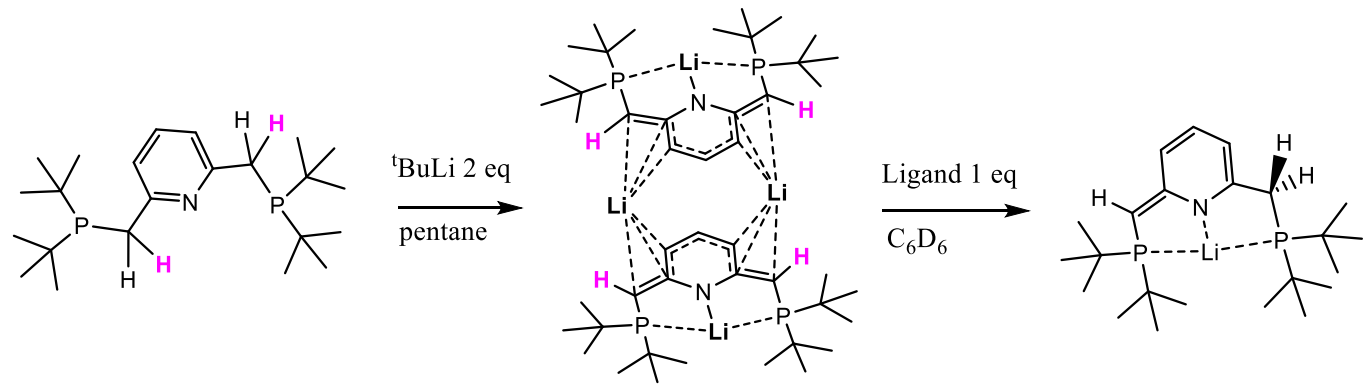

Figure 1.12. Lithium ion aggregates formed during the reaction of $t \mathrm{BuLi}$ with related PNP pincer systems. $^{51}$ 
A. Gers-Barlag reported attempts for the synthesis of a series of ligands represented in Figure 1.14. However, the synthesis of the ligand when the substituent was different from $t B u$ led to very small yields or was simply not possible. The isolation of VIII(iPr) was challenging as it was not possible to purify the product by silica column chromatography or by crystallization but only by precipitation from a concentrated pentane solution after months at low temperature. ${ }^{52}$

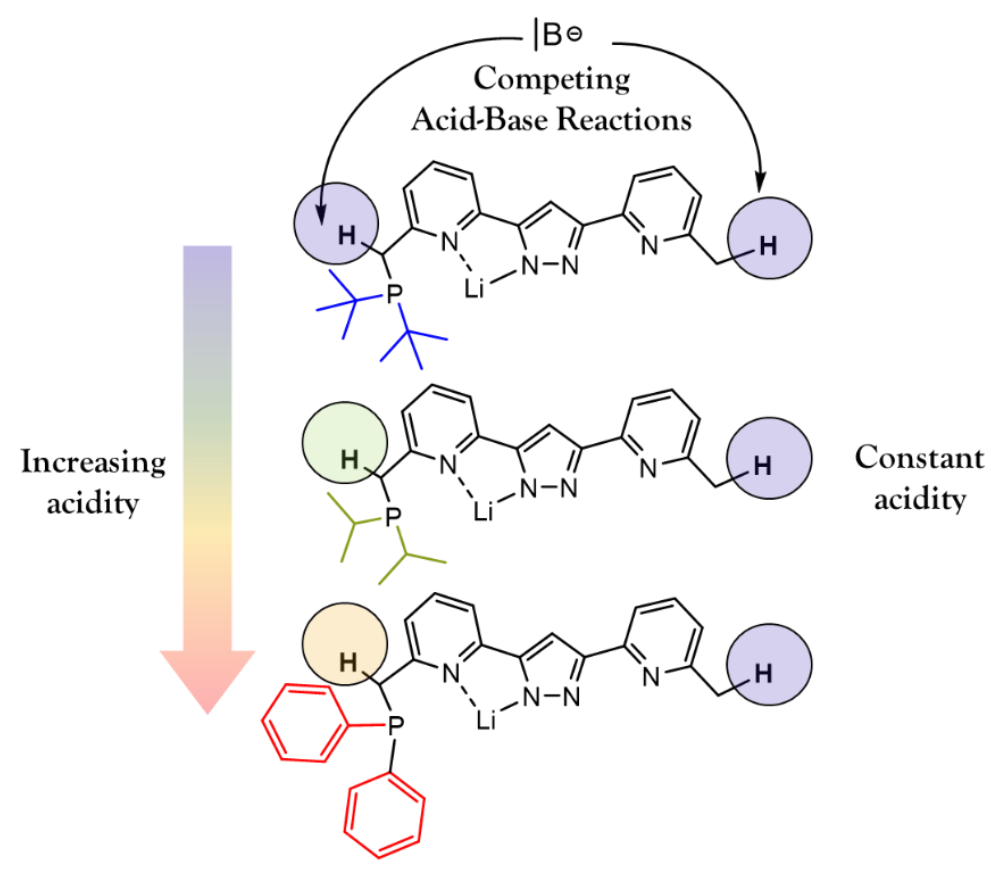

Figure 1.13. Synthetic problem of the Two-In-One pincers; The nature of substituents on the phosphorous atom possibly changes the acidity of the $-\mathrm{CH}_{2}$ and hampers the deprotonation of the $-\mathrm{CH}_{3}$ group.

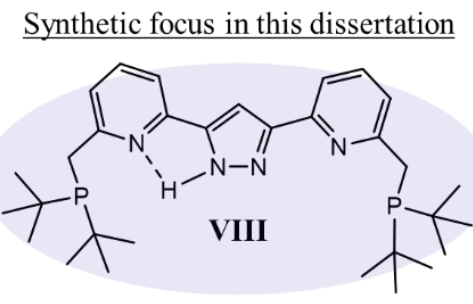

Total yield: $4 \%$

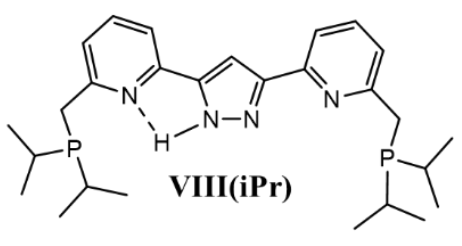

Total yield: $<1 \%$

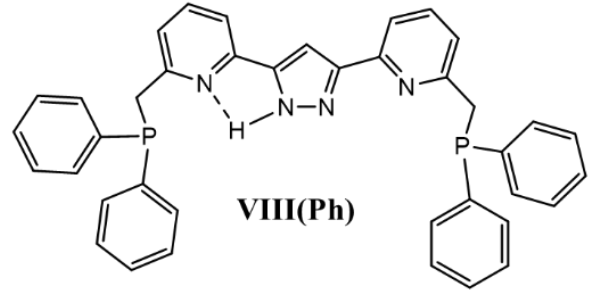

Not synthesized

Figure 1.14. Scope of different "Two-In-One" pincer ligands with different substituent on the phosphorous atom.

As the synthesis of VIII was synthetically limited by the nature of substituents on the phosphorus atom, this dissertation focused on the synthesis of complexes based on the ligand VIII, which could be obtained with a total yield of $4 \%$ (cf experimental section). 


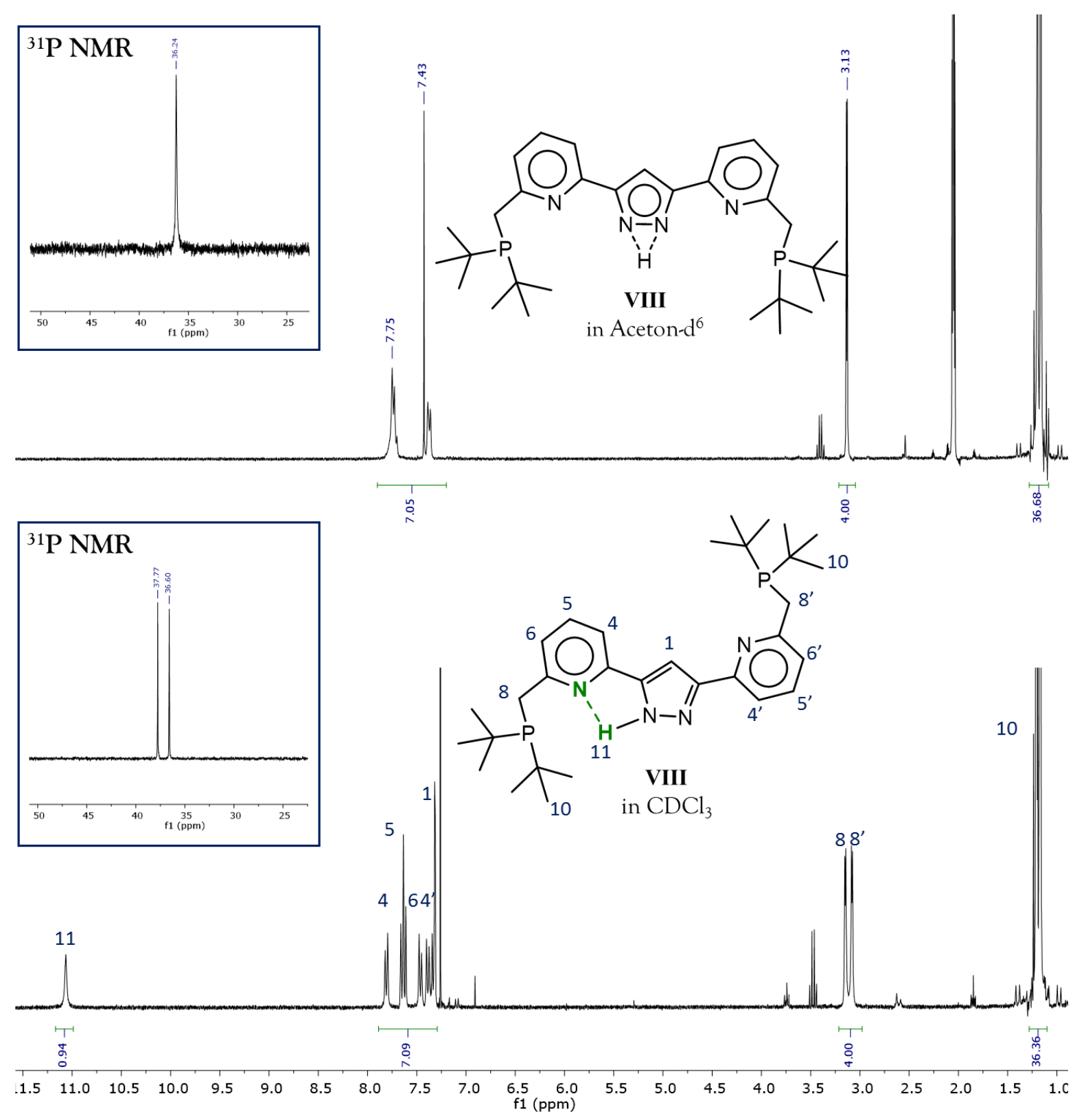

Figure 1.15. ${ }^{1} \mathrm{H}$ NMR $(300 \mathrm{MHz})$ and ${ }^{31} \mathrm{P}$ NMR $(121 \mathrm{MHz})$ spectra of VIII in $\mathrm{CDCl}_{3}$ (bottom) and in acetone- $\mathrm{d}^{6}$ (top) at $298 \mathrm{~K}$. The absence of prototropy is responsible for the inequivalent ${ }^{1} \mathrm{H}$ and ${ }^{31} \mathrm{P}$ resonances of each halves of the ligand.

VIII was analysed by ${ }^{1} \mathrm{H}$ and ${ }^{31} \mathrm{P}$ NMR spectroscopy (Figure 1.15). When VIII was measured in $\mathrm{CDCl}_{3}$, a clear segregation of the two inequivalent pyridine NMR signals was observed. However, in acetone- $\mathrm{d}^{6}$, the NMR signals of the pyridine were equivalent and broad. The proton on one nitrogen of the pyrazole could migrate to the adjacent nitrogen of the pyrazole in respect to prototropy. This phenomenon has been investigated by Samanta ${ }^{114}$ and Gers-Barlag ${ }^{52}$ in deuterated acetone- $\mathrm{d}^{6}$ where they observed coalescence of the NMR signals at room temperature. A discussion of the $\mathrm{N}-\mathrm{H}$ prototropy in pyrazole ligands has been made by K. Dalle. ${ }^{53}$ Fast prototropy was observed in polar solvent and led to broad $\mathrm{N}-\mathrm{H}$ resonances and localized intramolecular N-H...N hydrogen bond..$^{53}$ With non-polar solvent, prototropy was slower and gave rise to sharp N-H resonance as the hydrogen atom was localized on the NMR timescale. ${ }^{53} \mathrm{~A}$ 
hydrogen bond in VIII was characterized by an interatomic distance between the pyrazole and pyridine nitrogen atoms of $2.630 \AA$ according to the published crystal structure. ${ }^{114}$ The hydrogen bond likely slowed the rotation around the C-C bonds between the pyrazole and one pyridine and possibly contributed to the segregation of two resonance set for each halves of the ligand.

In conclusion, VIII was a ligand that could be obtained in seven steps as clean material, but its synthesis was limited by the possible occurrence of a side reaction which hampered the extension to other ligand systems.

\subsection{Study of the homobimetallic complex $\left[\mathrm{LH}_{2} \mathrm{Ni}_{2}(\mathrm{acac})_{2}\left(\mathrm{CH}_{3} \mathrm{CN}\right)\right] \mathrm{PF}_{6}(\mathbf{1})$}
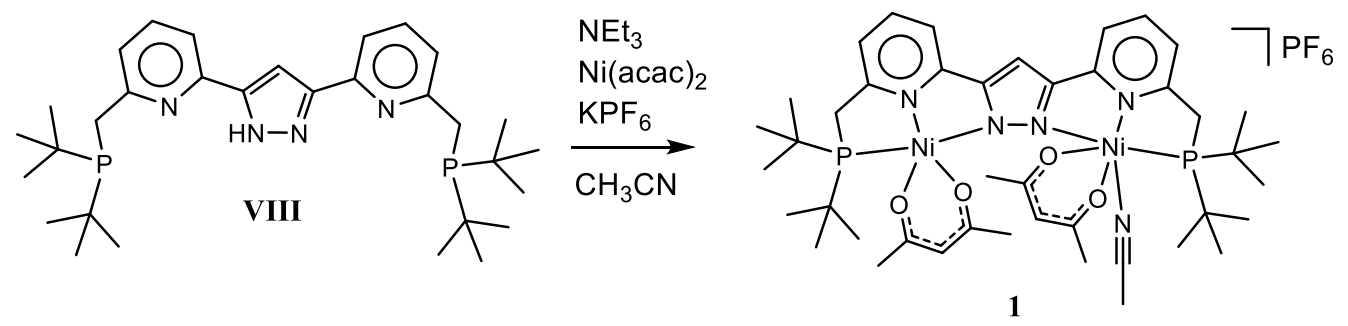

Figure 1.16. Synthesis of complex 1

VIII was suspended in dry acetonitrile, and the subsequent addition of $\mathrm{Ni}$ (acac) 2 led to complete dissolution of the ligand powder with a colour change to green, corresponding to the binding of the first $\mathrm{Ni}^{2+}$ ion. After addition of an excess of triethylamine, the colour of the solution became deeper green, which was consistent with the chelation of the second equivalent of $\mathrm{Ni}^{2+}$ giving the bimetallic complex $\left[\mathrm{LH}_{2} \mathrm{Ni}_{2}(\mathrm{acac})_{2}\left(\mathrm{CH}_{3} \mathrm{CN}\right)\right]^{+}$. $\mathrm{KPF}_{6}$ was added and the solution was stirred for several hours. It was filtered and then crystallized as deep green crystals suitable for X-ray diffraction analysis. Even if the acetylacetonate in the nickel salt could play the role of an internal base, an excess of external base (triethylamine) was needed for the quantitative deprotonation of the $\mathrm{N}-\mathrm{H}$ of the pyrazole.
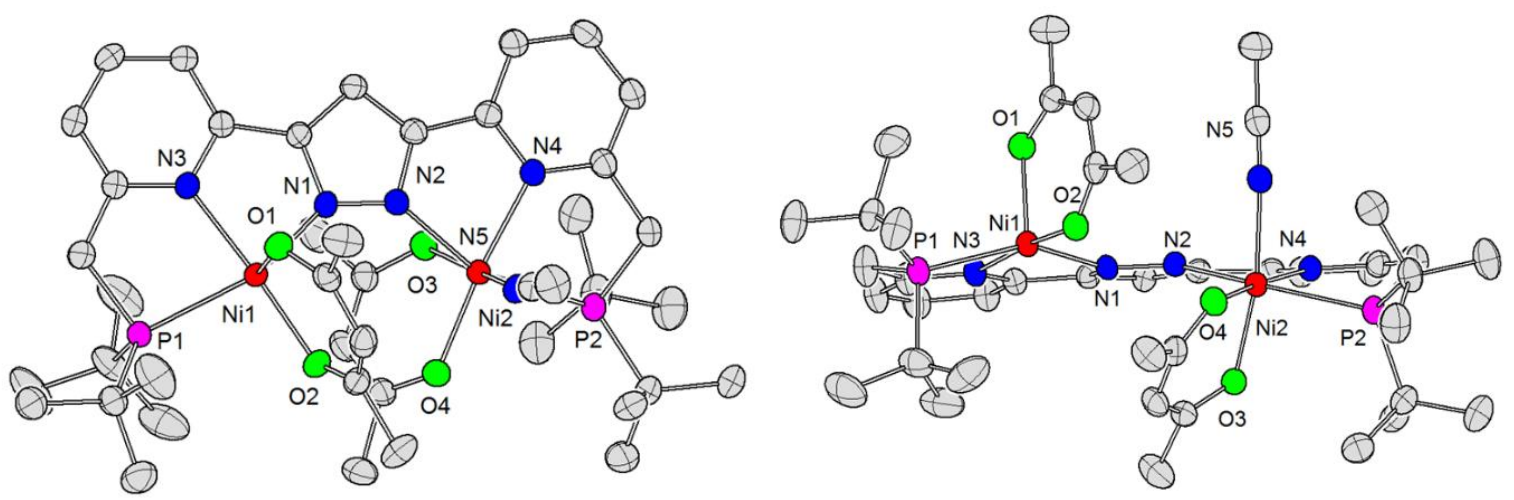

Figure 1.17. Molecular structure (thermal displacement ellipsoids shown at $50 \%$ probability) of the cation of complex 1. Hydrogen atoms and anions have been omitted for clarity. Left: top view of the molecular structure. Right: front view of the molecular structure 


\begin{tabular}{|c|c|c|c|}
\hline \multicolumn{2}{|c|}{ Bond Lengths around $\mathrm{Ni}(1) / \AA$} & \multicolumn{2}{|c|}{ Bond lengths around $\mathrm{Ni}(2) / \AA$} \\
\hline $\mathrm{Ni}(1)-\mathrm{O}(1)$ & $1.9743(16)$ & $\mathrm{Ni}(2)-\mathrm{O}(4)$ & $2.0085(16)$ \\
\hline $\mathrm{Ni}(1)-\mathrm{O}(2)$ & $1.9785(16)$ & $\mathrm{Ni}(2)-\mathrm{O}(3)$ & $2.0410(15)$ \\
\hline $\mathrm{Ni}(1)-\mathrm{N}(1)$ & $2.0358(19)$ & $\mathrm{Ni}(2)-\mathrm{N}(2)$ & $2.1405(19)$ \\
\hline $\mathrm{Ni}(1)-\mathrm{N}(3)$ & $2.0812(19)$ & $\mathrm{Ni}(2)-\mathrm{N}(4)$ & $2.0796(19)$ \\
\hline \multirow[t]{2}{*}{$\mathrm{Ni}(1)-\mathrm{P}(1)$} & $2.3972(7)$ & $\mathrm{Ni}(2)-\mathrm{P}(2)$ & $2.4780(7)$ \\
\hline & & $\mathrm{Ni}(2)-\mathrm{N}(5)$ & $2.107(2)$ \\
\hline
\end{tabular}

Table 1.1 Selected bond lengths

Interestingly, the crystal structure showed two inequivalent Ni centers; both were coordinated by an acetylacetonate moiety but one of them had an additional solvent molecule which gave rise to one pentacoordinate $\mathrm{Ni}(1)$ and one hexacoordinate $\mathrm{Ni}(2)$ metal ions. The two nitrogen atoms of the pyrazole moiety were coordinated to their corresponding nickel atoms with different bond lengths. The Ni(1)-N(1) bond was longer (2.036 $)$ than the $\mathrm{Ni}(2)-\mathrm{N}(2)$ one (2.141 $⿱$ ). The large distance difference between $\mathrm{Ni}(1)-\mathrm{P}(1)=2.397 \AA$ and $\mathrm{Ni}(2)-\mathrm{P}(2)=2.478 \AA$ particularly attested the known plasticity of the chemical bound between a phosphorus atom and a metal (Table 1.1. highlighted in red). Those metrical differences are correlated to the geometric differences (ie square pyramid and octahedral geometries) adopted by the respective $\mathrm{Ni}^{2+}$ ions.

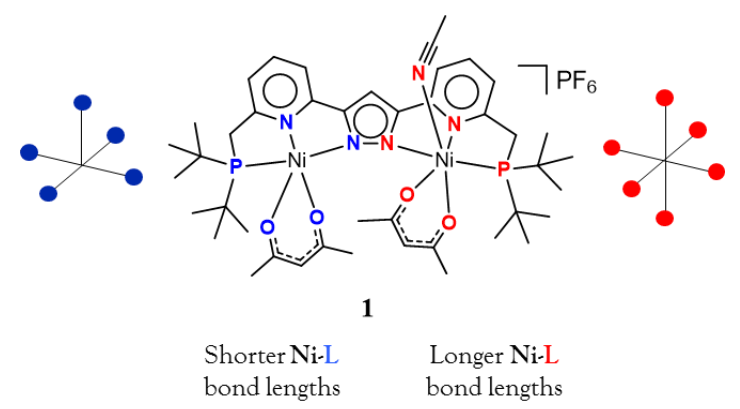

Figure 1.18. Different geometries adopted by nickel ions of complex $\mathbf{1}$ in the solid state.

The spin state of complex $\mathbf{1}$ was analysed by SQUID (Superconducting QUantum Interference Device) magnetometry. A measurement in the solid state revealed a plateau at $\chi_{\mathrm{m}} \mathrm{T}=2.15$ $\mathrm{cm}^{3} \cdot \mathrm{mol}^{-1} \cdot \mathrm{K}$ from 100 to $298 \mathrm{~K}$ (Figure 1.19 ). Decreasing the temperature to $2 \mathrm{~K}$ revealed antiferromagnetic coupling between the two nickel centers. Simulation using an isotropic Heisenberg Dirac Van Vleck Hamiltonian including a Zeeman term gave a magnetic exchange coupling parameter of $J=-3.6 \mathrm{~cm}^{-1}$ and $\mathrm{g}=2.48$ and a contribution from $35 \%$ of paramagnetic impurities.

$$
\widehat{\mathrm{H}}=-2 \int \widehat{\mathrm{S}_{1}} \cdot \widehat{\mathrm{S}_{2}}+g \mu_{\mathrm{B}}\left(\overrightarrow{\mathrm{S}_{1}}+\overrightarrow{\mathrm{S}_{2}}\right) \overrightarrow{\mathrm{B}}
$$


Since the material utilized for the measurement was crystalline and the Lande factor $g$ was unusually high, the above result was not consistent. Hence the data were simulated with another model including ZFS (zero field splitting).

$$
\widehat{\mathrm{H}}=g \mu_{\mathrm{B}}\left(\overrightarrow{\mathrm{S}_{1}}+\overrightarrow{\mathrm{S}_{2}}\right) \overrightarrow{\mathrm{B}}+\mathrm{D} \sum_{i=1}^{2}\left[\widehat{\mathrm{S}}_{\mathrm{zi}}^{2}-\frac{\mathrm{S}(\mathrm{S}+1)}{3}\right]
$$

The result was interpreted as two nickel atoms having two different ZFS: $D_{1}=50.5 \mathrm{~cm}^{-1}$ and $\mathrm{D}_{2}$ $=15.2 \mathrm{~cm}^{-1}$ with identical $\mathrm{g}$ values $=2.01$. The fact that two different ZFS was observed for the two nickel(II) ions would be consistent with their different coordination environments in the solid state, as previously mentioned.

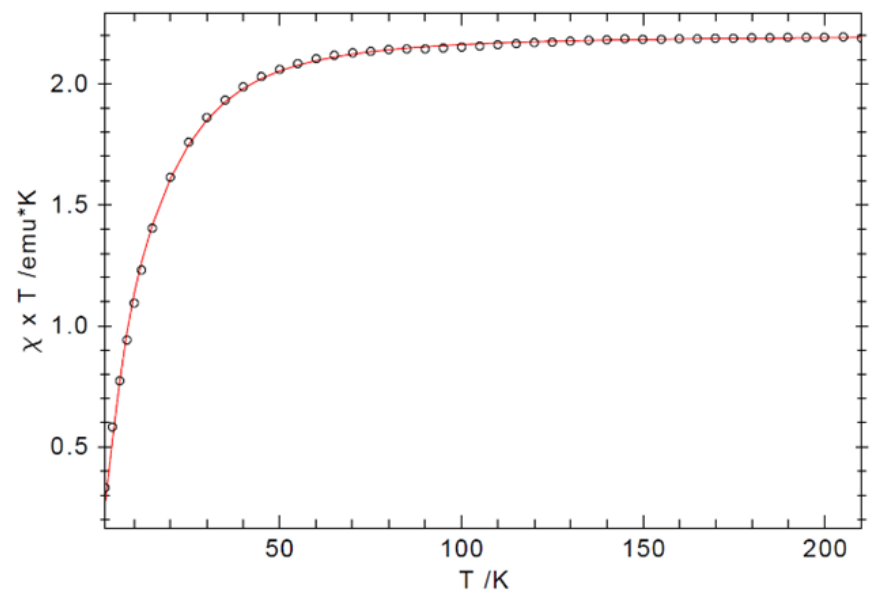

Figure 1.19. Temperature dependence of $\chi_{\mathrm{m}} \mathrm{T}$ of complex $\left[\mathrm{LH}_{2} \mathrm{Ni}_{2}(\mathrm{acac})_{2}\left(\mathrm{CH}_{3} \mathrm{CN}\right)\right] \mathrm{PF}_{6} . \mathrm{D}_{1}=50.5 \mathrm{~cm}^{-1}, \mathrm{D}_{2}=15.2 \mathrm{~cm}^{-1}, \mathrm{~g}=2.01$.

The data collected by SQUID for complex $\mathbf{1}$ in the solid state clearly indicated the absence of spin transitions. The magnetic behaviour was then studied in solution since it may be different from the solid state. The possibility of molecular reorganization of the acetylacetonato ligands in complex 1 could lead to a non-trivial coordination mode in a $S=0$ form represented in Figure 1.20. The acetylacetonato ligand was sometimes able to adopt unusual binding motifs with transition metal complexes. ${ }^{54}$ Thus, the properties of complex $\mathbf{1}$ in solution were investigated.

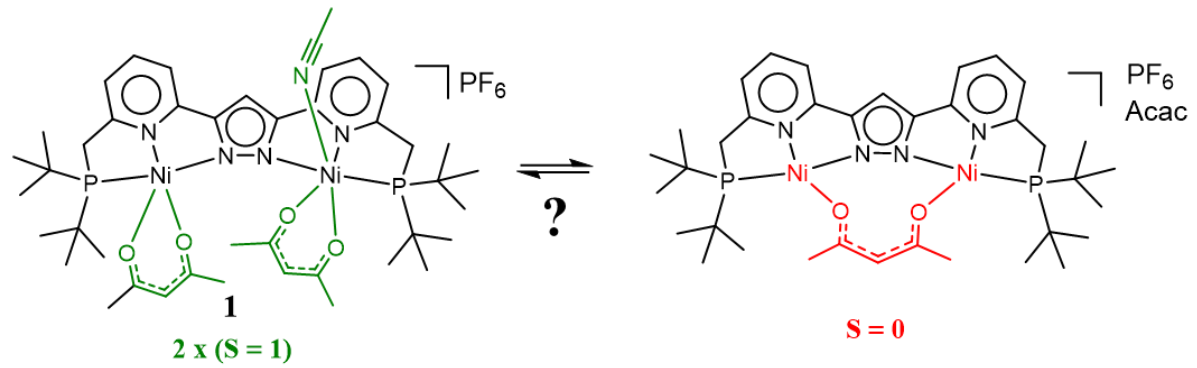

Figure 1.20. Hypothetical spin transition in complex $\mathbf{1}$ caused by ligand dissociation.

${ }^{1} \mathrm{H}$ and ${ }^{31} \mathrm{P}$ NMR spectra of complex 1 were recorded at different temperatures. The ${ }^{1} \mathrm{H}$ NMR spectrum of 1 in $\mathrm{CD}_{2} \mathrm{Cl}_{2}$ at $298 \mathrm{~K}$ is represented in Figure 1.21 and showed paramagnetic shifted 
${ }^{1} \mathrm{H}$ NMR signals spread from -29 to $58 \mathrm{ppm}$. The ${ }^{31} \mathrm{P}$ NMR spectrum at $298 \mathrm{~K}$ showed a resonance for the $\mathrm{PF}_{6}{ }^{-}$anion and a hardly detectable broad signal at $82 \mathrm{ppm}$ (Figure 1.23). Two-dimensional experiments $\left({ }^{1} \mathrm{H} /{ }^{1} \mathrm{H}\right.$ COSY $)$ did not show cross-peak correlations due to fast nuclei relaxations. However, ${ }^{13} \mathrm{C}$ NMR and ${ }^{1} \mathrm{H} /{ }^{13} \mathrm{C}$ HSQC spectra could be recorded, in which $-\mathrm{CH}_{\mathrm{n}}$ resonances range from -176 to $625 \mathrm{ppm}$ (cf experimental section). The Evans method was utilized at different temperatures affording $\mu_{\text {eff }}=4.36 \mu_{\mathrm{B}}$ with the following equation: $:^{55}$

$$
\chi_{\mathrm{g}}=\left|\frac{3 \Delta \mathrm{f}}{4 \pi \mathrm{fm}}\right|+\chi_{0}
$$

$\chi_{g}$ was the mass susceptibility, $f$ the frequency of the spectrometer, $m$ the mass of the substance, $\Delta \mathrm{f}$ the observed chemical shifts, $\chi_{0}$ the mass susceptibility of the solvent. This value was close from the theoretical spin only value for two uncoupled $\mathrm{Ni}^{2+}$ centers with a spin quantum number $\mathrm{S}=$ 1 each. (For a Landé factor $g=2.1$, theoretical $\mu_{\text {eff }}=g_{L} \sqrt{\sum S_{i}\left(S_{i}+1\right)} \mu_{B}=4.2 \mu_{B}$ ). Those magnetic values were similar to the experimental values found in the solid state $\left(4.2 \mu_{\mathrm{B}}\right)$.

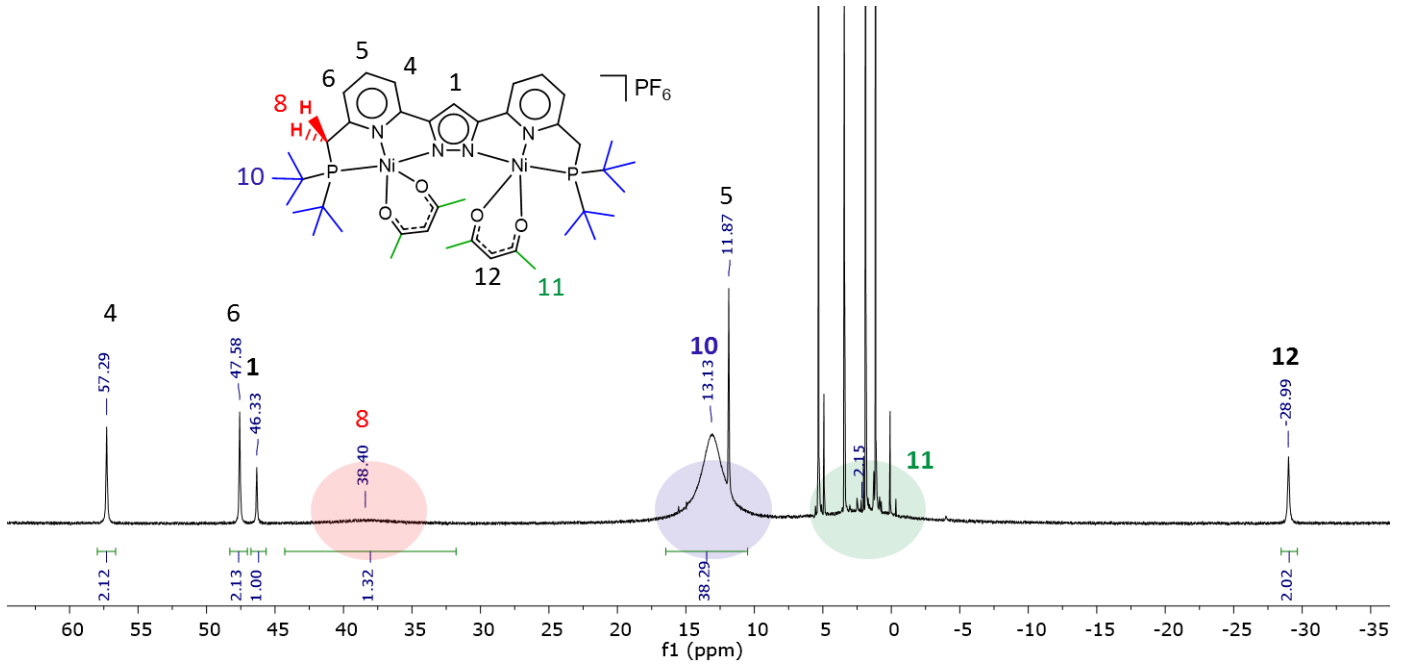

Figure 1.21. ${ }^{1} \mathrm{H}$ NMR spectrum of complex 1 in $\mathrm{CD}_{2} \mathrm{Cl}_{2}(400 \mathrm{MHz}), 298 \mathrm{~K}$. Protons that show coalescence (Figure 1.22) are marked with colours. 


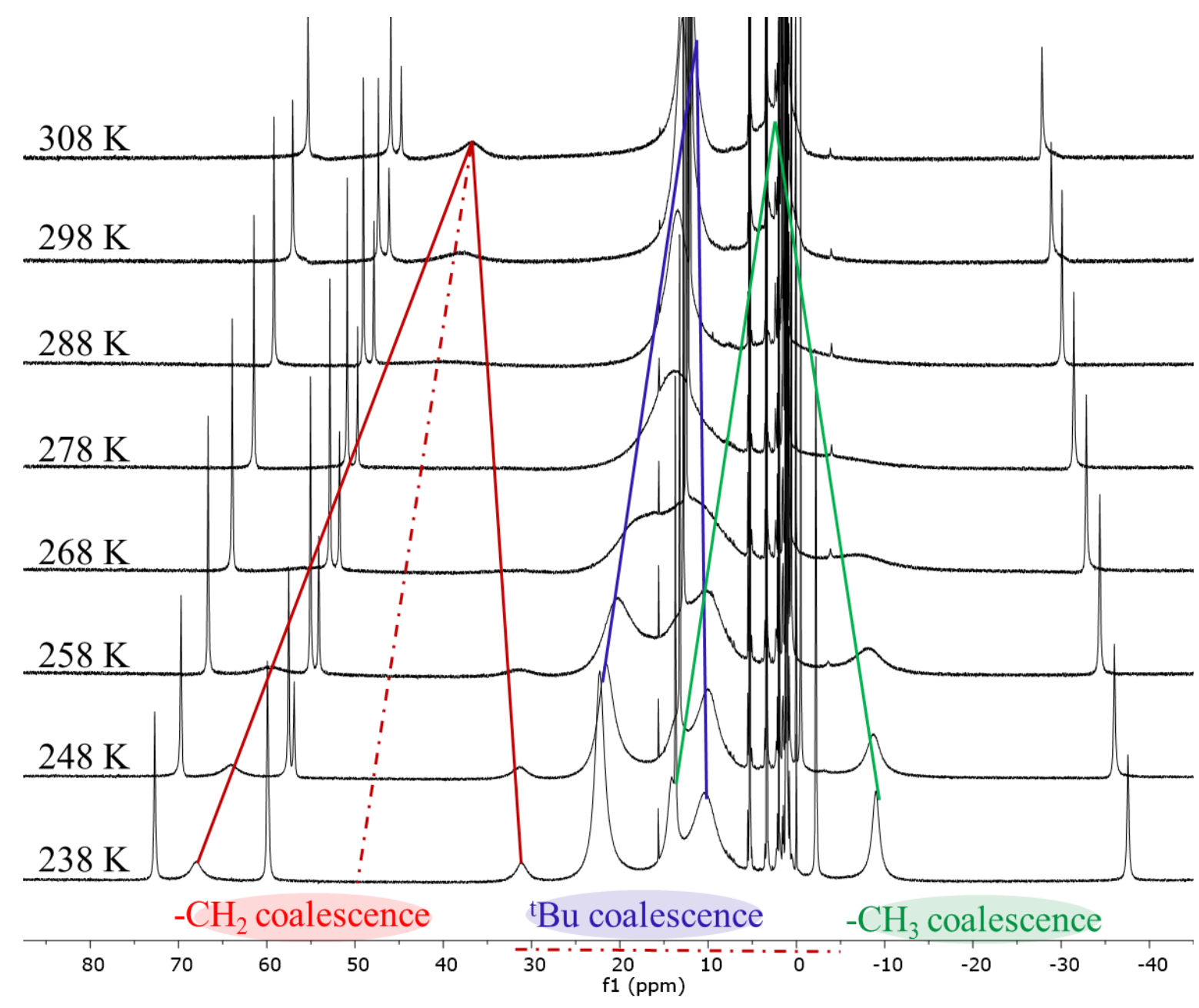

Figure 1.22. VT ${ }^{1} \mathrm{H}$ NMR spectra of complex 1 in $\mathrm{CD}_{2} \mathrm{Cl}_{2}(400 \mathrm{MHz})$.

${ }^{1} \mathrm{H}$ NMR (Figure 1.22) and ${ }^{31} \mathrm{P}$ NMR (Figure 1.23) spectra recorded at variable temperatures from $238 \mathrm{~K}$ to $308 \mathrm{~K}$ revealed a linear dependence between $1 / \mathrm{T}$ and the chemical shifts in complex $\mathbf{1}$. Hence, those chemical shifts followed the Curie law. The Curie behaviour indicated by extrapolation, that the complex did not undergo spin transitions (i.e. the Evans calculation method gave the same $\mu_{\text {eff }}$ values at different temperatures). The intensity of the ${ }^{31} \mathrm{P}$ NMR signals notably increased when the temperature decreased (Figure 1.23). Complex 1 displayed an interesting molecular dynamic: three resonances of the complex showed coalescence. The coalescence of those resonances happened at the same temperature $(278 \mathrm{~K})$, which was a hint that they originate from the same chemical process (Figure 1.22). A coalescence phenomenon for $\mathrm{T}_{\mathrm{d}}$ paramagnetic $\mathrm{Ni}^{2+}$ complexes have also been investigated in 2011 (Figure 1.24). ${ }^{56}$ 


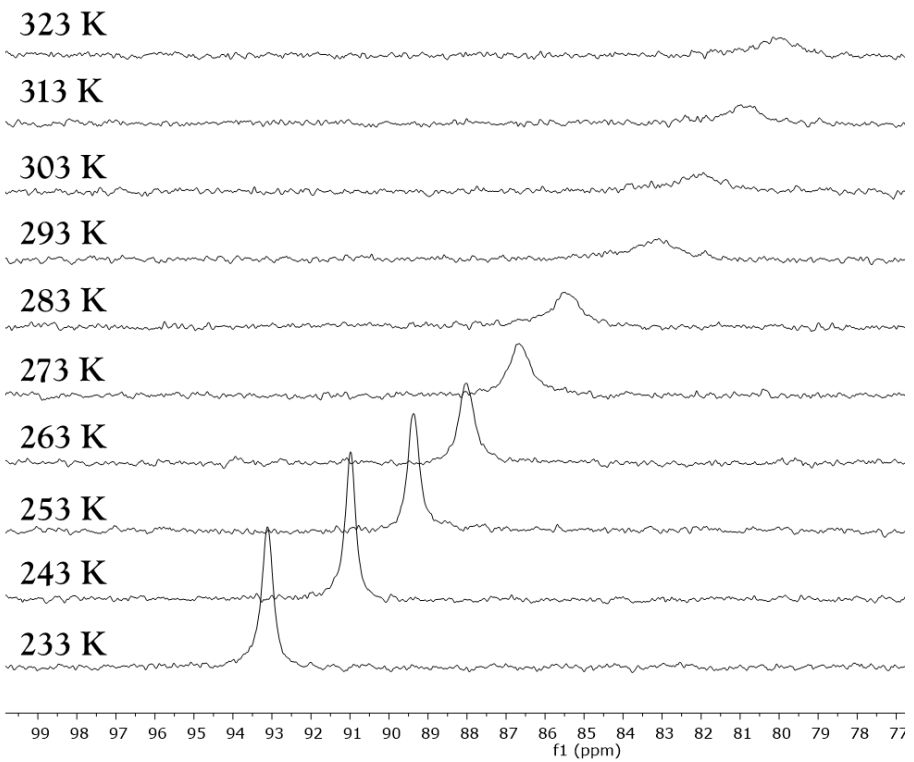

Figure 1.23 VT ${ }^{31} \mathrm{P}$ NMR of complex 1 in $\mathrm{CD}_{2} \mathrm{Cl}_{2}(162 \mathrm{MHz})$.
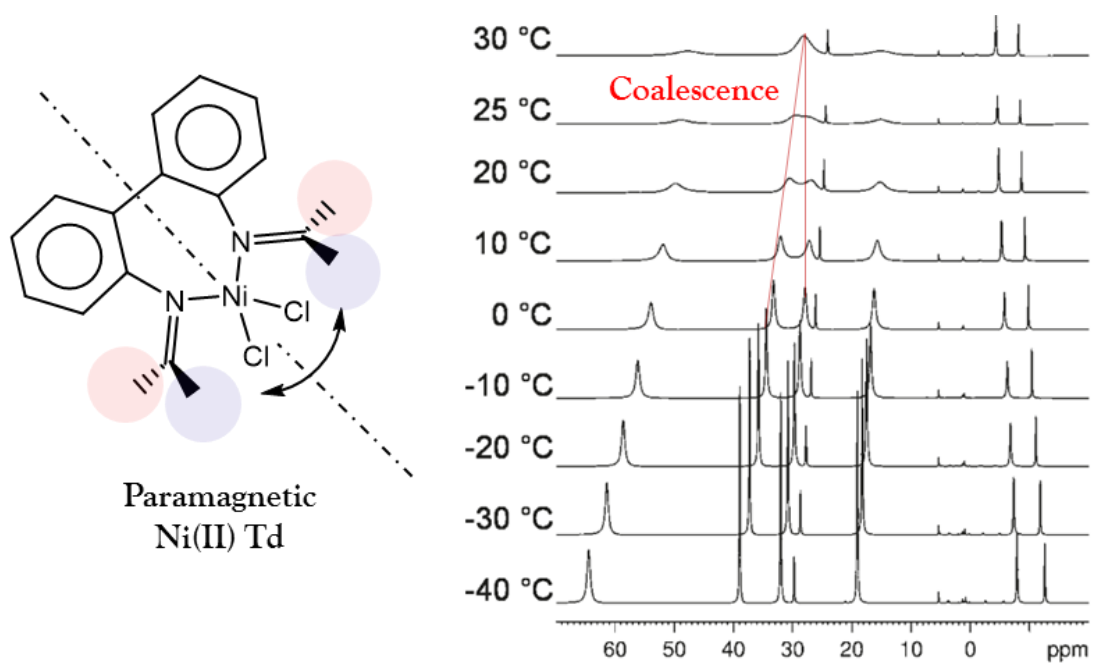

Figure 1.24. ${ }^{1} \mathrm{H}$ NMR study of a paramagnetic $\mathrm{T}_{\mathrm{d}}$ high spin $\mathrm{Ni}^{2+}$ complex. ${ }^{56}$

The coalescence in complex 1 was likely due to the slow motion of the acetylacetonate. At room temperature, the acetylacetonate flipped up and down the plane described by the pyrazolate moiety, so that its position was averaged on the NMR timescale. Lowering the temperature slowed the motion of the swivelling acetylacetonates so that the chemical environment above and under the plane described by the pyrazolate became different. Thus, at low temperature, enantiomers of complex $\mathbf{1}$ did not interconvert anymore, which led to the observation of the diastereotopic protons in the NMR spectra (Figure 1.22). Such phenomena are well established in pyrazolate based dinuclear complexes. ${ }^{57,58}$

Although all NMR signals of complex $\mathbf{1}$ followed the Curie law, the acetonitrile resonance singularly differed from this trend. It is worth mentioning that acetonitrile was present in stoichiometric amount as there was only one acetonitrile in the elementary cell (cf experimental 
section and NMR spectrum in Figure 1.22). The acetonitrile molecule was bound to nickel in the crystal structure of complex 1 but was apparently released in the solvent upon dissolution. At room temperature, the chemical shift of acetonitrile $(1.92 \mathrm{ppm})$ was close from the free acetonitrile shift in $\mathrm{CD}_{2} \mathrm{Cl}_{2}(1.97 \mathrm{ppm})^{59}$ (Figure 1.25). Upon decreasing the temperature, the signal was shifted upfield, from $-0.5 \mathrm{ppm}$ at $248 \mathrm{~K}$ to $-2.1 \mathrm{ppm}$ at $238 \mathrm{~K}$. It did not follow the Curie law (Figure 1.27). This was a hint that a dynamic phenomenon was associated with the shift of the acetonitrile molecule. The negative shift values obtained below $248 \mathrm{~K}$ suggested the contribution of a hyperfine shift, likely coming from the paramagnetic metal. At lower temperature, the acetonitrile was closer from the nickel center, as the signal was paramagnetically shifted.

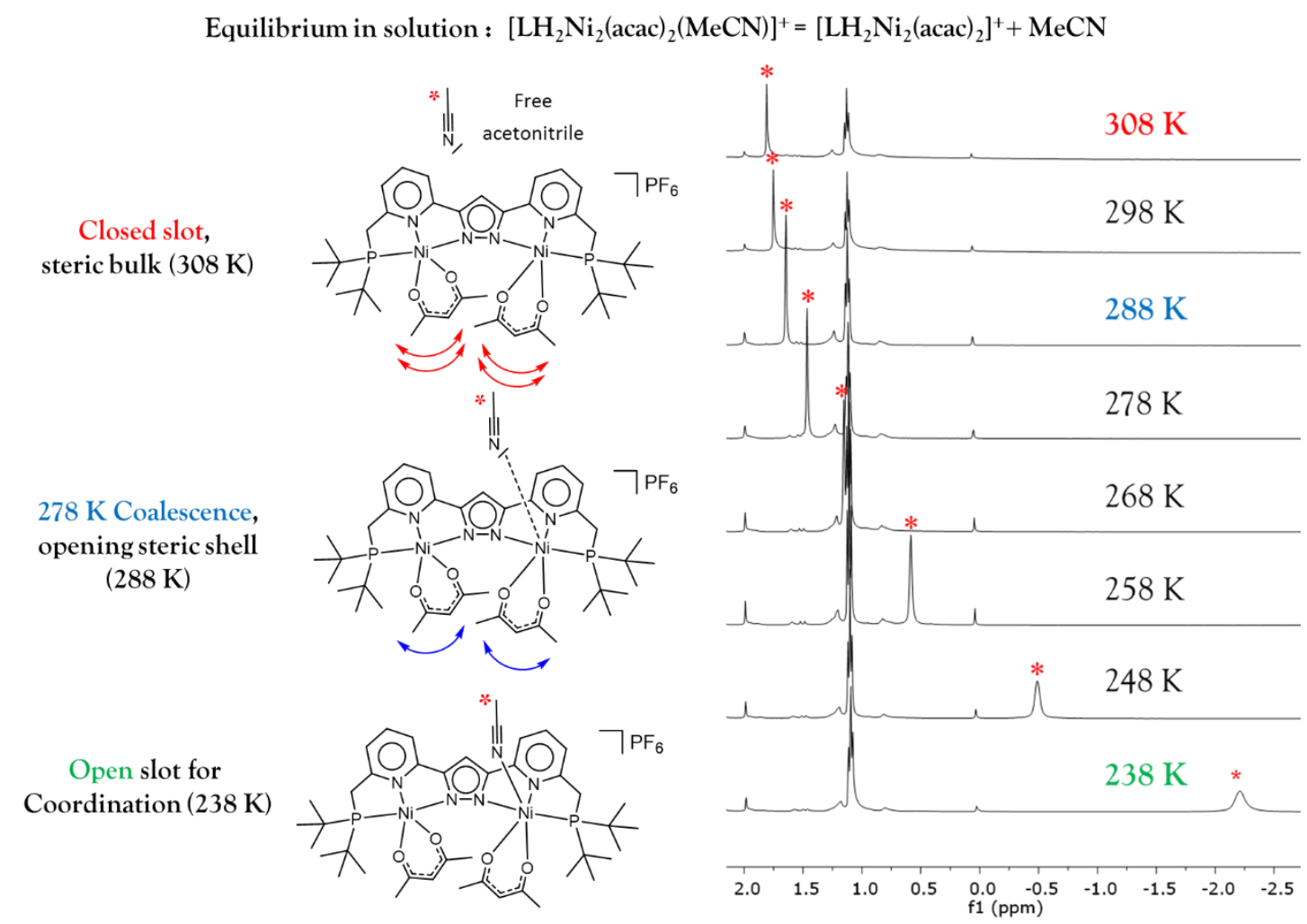

Figure 1.25. VT ${ }^{1} \mathrm{H}$ NMR of 1 in $\mathrm{CD}_{2} \mathrm{Cl}_{2}(400 \mathrm{MHz})$, from $2 \mathrm{ppm}$ to $-2.5 \mathrm{ppm}$. The acetonitrile resonance crosses the Curie asymptotic value of $1 / \mathrm{T}=0$. A dynamic process is associated to that shift.

To summarize the understanding so far of this complex in solution, an equilibrium for the reversible coordination of the acetonitrile molecule with complex $\mathbf{1}$ in solution was proposed in Figure 1.25. Once complex 1 was dissolved in dichloromethane, the acetonitrile which was bound to one nickel ion in the solid state left the coordination sphere, gave two equivalent high spin $\mathrm{Ni}^{2+}$ ions, with swivelling acetylacetonate moieties. The swivelling motion was responsible for the coalescence of ${ }^{1} \mathrm{H}$ NMR signals at $278 \mathrm{~K}$ (Figure 1.22). This motion created a steric crowd that favoured the exclusion of the acetonitrile ligand. When the temperature was decreased, the motion and the steric bulk caused by the acetylacetonate also decreased so that the free acetonitrile molecule was approaching the coordination sphere of the metal. It was inappropriate to state that the acetonitrile bound the nickel ion at low temperature since there was no evidence 
for that. A reason supporting this idea was that the coordination of acetonitrile would cause complex 1 to have different ${ }^{1} \mathrm{H}$ and ${ }^{31} \mathrm{P}$ chemical shifts for each halves of the complex, which was actually not observed. Even at $238 \mathrm{~K}$, each half of complex 1 were equivalent (Figure 1.22).

Since all resonances of complex 1 followed the Curie law, the shifts were plotted in a graph in order to extract the hyperfine data (Figure 1.24)..$^{60,61,62}$ This method is routinely used for paramagnetic complexes which now allows the characterization of single molecule magnets. ${ }^{63,64}$ Paramagnetic $\mathrm{Ni}^{2+}$ complexes ${ }^{65,66}$ are valuable benchmark systems for the improvement of NMR methods allowing the characterization of biomolecules. ${ }^{67}$ The Evan's method gives access to the magnetic susceptibility and thus, the number of unpaired electrons. However, a plot of $\delta=f(1 / T)$ deliver additional information. Indeed, the temperature dependent NMR chemical shifts can be obtained by in the sum of the orbital shift $\left(\delta^{\text {orb }}\right)$ and hyperfine shift $\left(\delta^{\mathrm{HF}}\right)$ :

$$
\delta_{\mathrm{T}}^{\mathrm{obs}}=\delta^{\mathrm{orb}}+\delta_{\mathrm{T}}^{\mathrm{HF}}
$$

The orbital shift $\left(\delta^{\text {orb }}\right)$ is a temperature independent diamagnetic contribution. This parameter can be estimated by the extrapolation of the limit $1 / \mathrm{T}=0$ with the intercept of the $\mathrm{Y}$ axis from Curie plots (Figure 1.24). The term $\delta^{\mathrm{HF}}$ is the hyperfine shift and can be divided in two subsequent terms, a Fermi contact term $\left(\delta^{\mathrm{FC}}\right)$ and a pseudocontact $\left(\delta^{\mathrm{PC}}\right)$ term.

$$
\delta^{\mathrm{HF}}=\delta^{\mathrm{FC}}+\delta^{\mathrm{PC}}
$$

The Fermi contact shift $\delta^{\mathrm{FC}}$ is caused by the spin density of the unpaired electron on the metal which is delocalized through chemical bonds on proximal ligands. The pseudocontact shift $\delta^{\mathrm{PC}}$ is caused by the dipolar through space interaction between the magnetic moment of the unpaired electron of the metal and the magnetic moment of the observed nuclei. Such interaction has a strong dependence with the distance $\left(1 / \mathrm{r}^{3}\right)$ and can be neglected for nuclei distant from the paramagnetic center. Thus, the Fermi contact shift is usually the major contribution of the hyperfine shift and corresponds to the sign and amplitude of slopes in Curie plots. The Fermi contact is proportional to the spin density and can be written as follow:

$$
\delta^{\mathrm{FC}}=\frac{\mathrm{S}(\mathrm{S}+1) \beta_{\mathrm{e}}}{3 \mathrm{k}_{\mathrm{B}} \mathrm{T} \gamma_{\mathrm{N}}} \mathrm{gA}
$$

$S$ being the spin quantum number, $\beta_{\mathrm{e}}$ the bohr magneton, $\gamma_{\mathrm{N}}$ the gyromagnetic moment of the nuclei, $\mathrm{k}_{\mathrm{B}}$ the Bolzmann constant, $\mathrm{T}$ the temperature, $\mathrm{g}$ and $\mathrm{A}$ are the $\mathrm{g}$ - and A-hyperfine tensors. Paramagnetic NMR data are systematically interpreted with the support of DFT. In the present pre-study case, NMR experimental data were collected and analysed by Curie plots. Temperature independent $\delta^{\text {orb }}$ terms obtained in Figure 1.24 were unfortunately not in the diamagnetic range $(\delta<0 \mathrm{ppm})$. Such finding suggested a non-neglectable influence of ZFS, whereby a second order polynomial fitting could be applied in the Curie plot. ${ }^{65}$ 


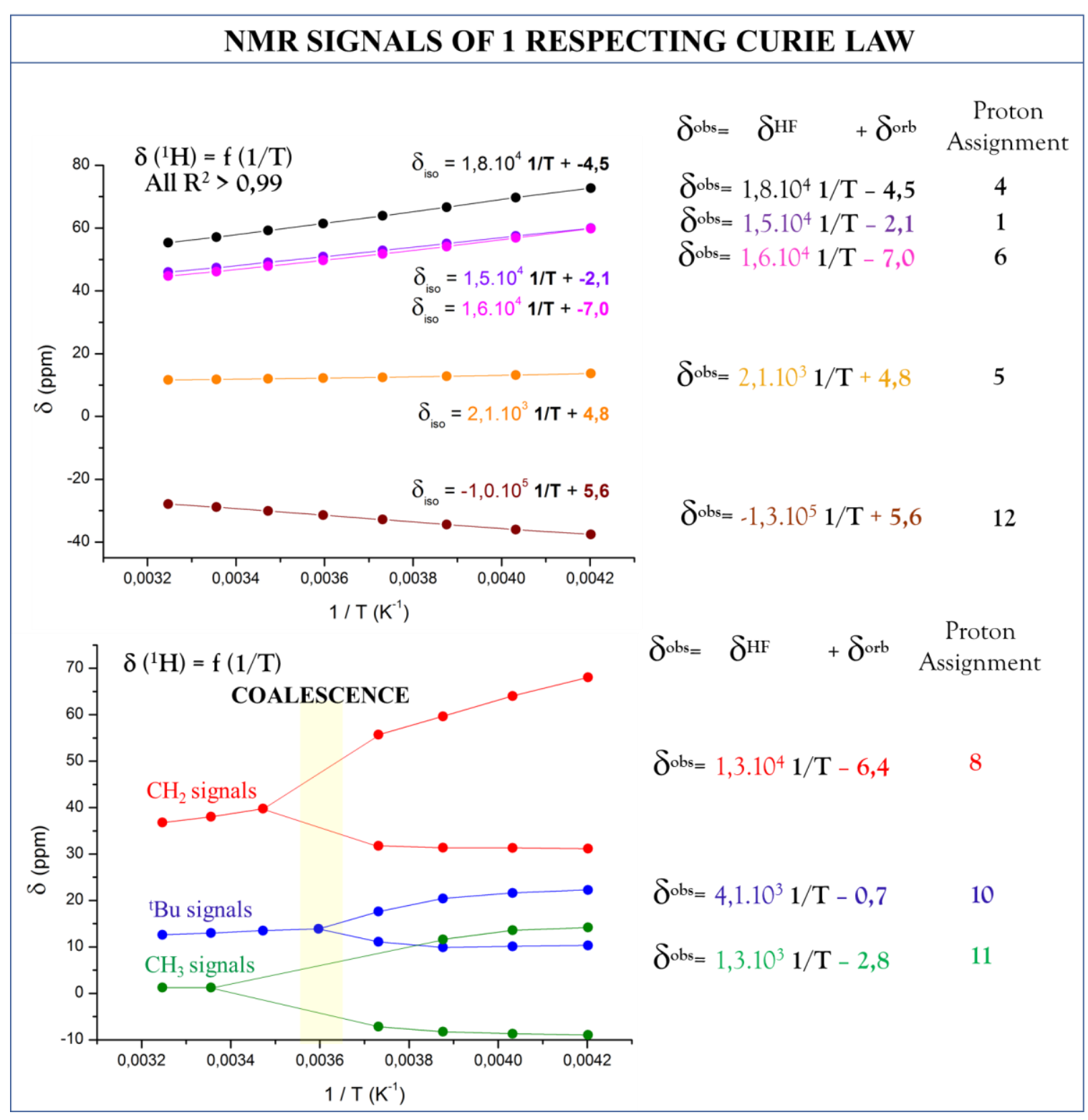

Figure 1.26. Curie plot of ${ }^{1} \mathrm{H}$ NMR resonances of complex 1 linearly fitted. Top plot involves the resonances that do not coalesce. Bottom plot involves the three resonances that coalesce at $278 \mathrm{~K}$. 


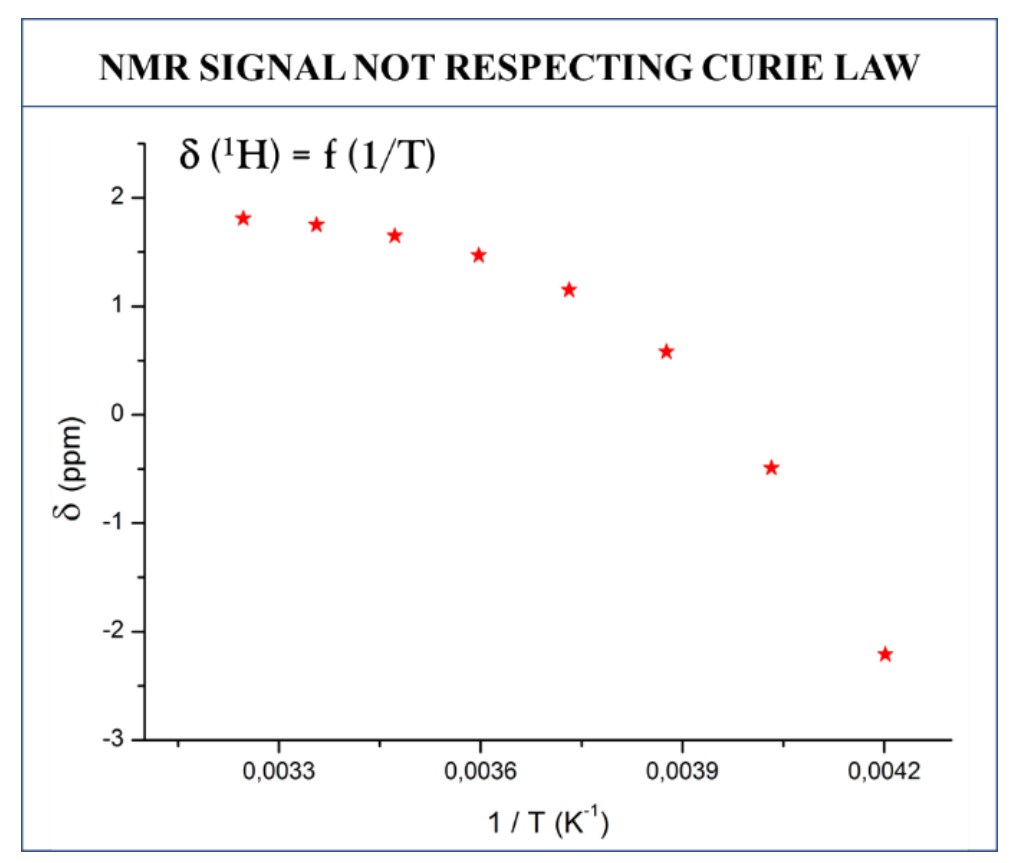

Figure 1.27. Curie plot of the ${ }^{1} \mathrm{H}$ resonance of the acetonitrile contained in complex $\mathbf{1}$.

The weak coordination strength of acetonitrile in complex 1 and its dissociation in solution was further suggested by ESI mass spectrometry. In Figure 1.28, one dominant peak located at $\mathrm{m} / z=$ $851.30 \mathrm{~m} / z$ was consistent with the molecular weight of the $\mathrm{LH}_{2} \mathrm{Ni}_{2}(\mathbf{a c a c})_{2}{ }^{+}$cation free from acetonitrile. However, it could not be excluded that acetonitrile was removed by the vacuum stage of the ionization process during the measurement.

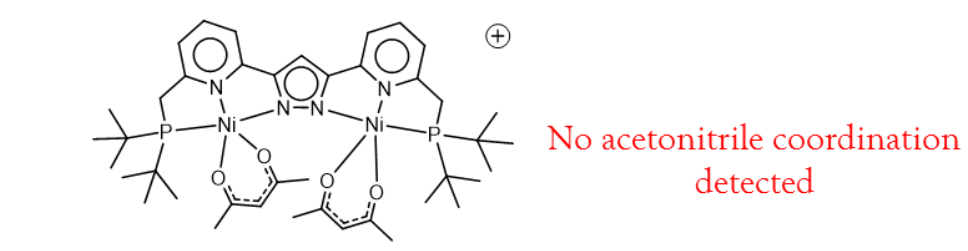

Molecular weight : $851.30 \mathrm{~g} \cdot \mathrm{mol}^{-1}$

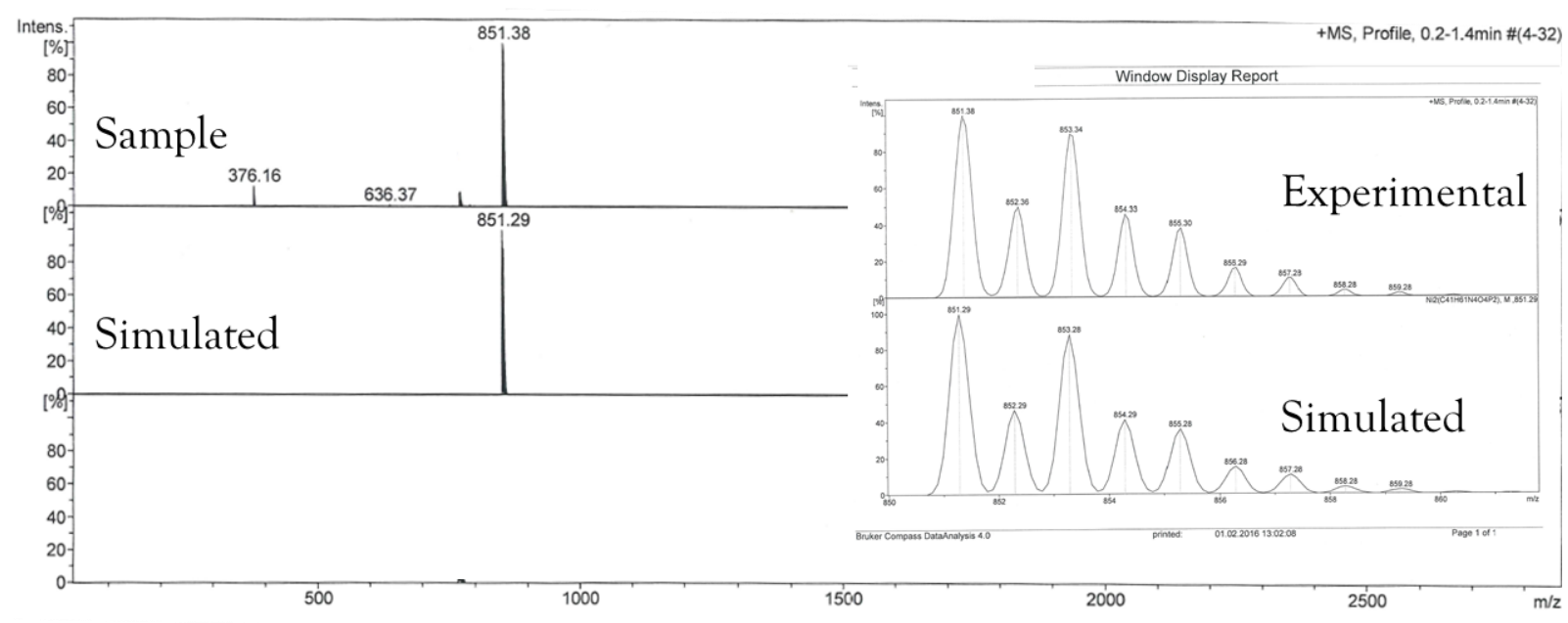

Figure 1.28. ESI mass spectrum of complex 1 in $\mathrm{CH}_{3} \mathrm{CN}$. The inset shows the experimental and simulated isotropic distribution pattern for $\mathrm{LH}_{2} \mathrm{Ni}_{2}(\text { acac })_{2}{ }^{+}$. 
I.4 Study of the homobimetallic $\left[\mathrm{LH}_{2} \mathrm{Ni}_{2}(\mathrm{Br})_{2}\right] \mathrm{PF}_{6}(2)$ complex.
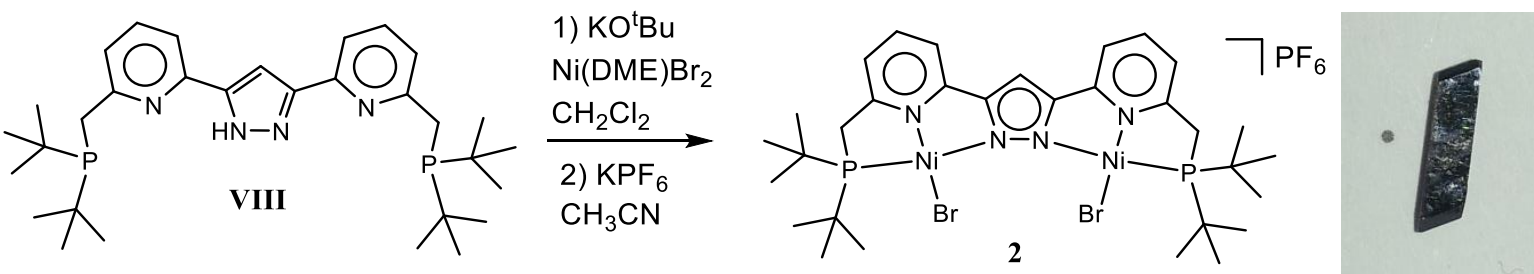

Figure 1.29. Synthesis of $\left[\mathrm{LH}_{2} \mathrm{Ni}_{2}\left(\mathrm{Br}_{2}\right] \mathrm{PF}_{6}\right.$. On the right: photography of a crystal of complex 2 $(\approx 1 \mathrm{~cm})$.

Complex 2 was first synthesized by Subhas Samanta. However, the synthesis of the complex required improvements. Thus, the following method was applied: VIII was dissolved in methylene chloride and one equivalent of $\mathrm{Ni}^{2+}$ bromide ethylene glycol dimethyl ether $\mathrm{Ni}(\mathrm{DME}) \mathrm{Br}_{2}$ was added. The suspension turned red after several minutes. Subsequently, a stoichiometric amount of $\mathrm{KOtBu}$ was added and a second equivalent of $\mathrm{Ni}(\mathrm{DME}) \mathrm{Br}_{2}$ was added. After stirring for $30 \mathrm{~min}$, the solution became intense dark red consistent with the second metal binding. The cationic dinuclear complex was then treated with $\mathrm{KPF}_{6}$ in acetonitrile to exchange anions. The red powder was redissolved in methylene chloride and set to vapor diffusion with pentane, giving suitable crystals for X-ray diffraction analysis. The cation $\left[\mathrm{LH}_{2} \mathrm{Ni}_{2}(\mathrm{Br})_{2}\right]^{+}$has an apparent non-crystallographic $\mathrm{C}_{2}$ symmetry axis with two distorted square planar geometries around nickel atoms.
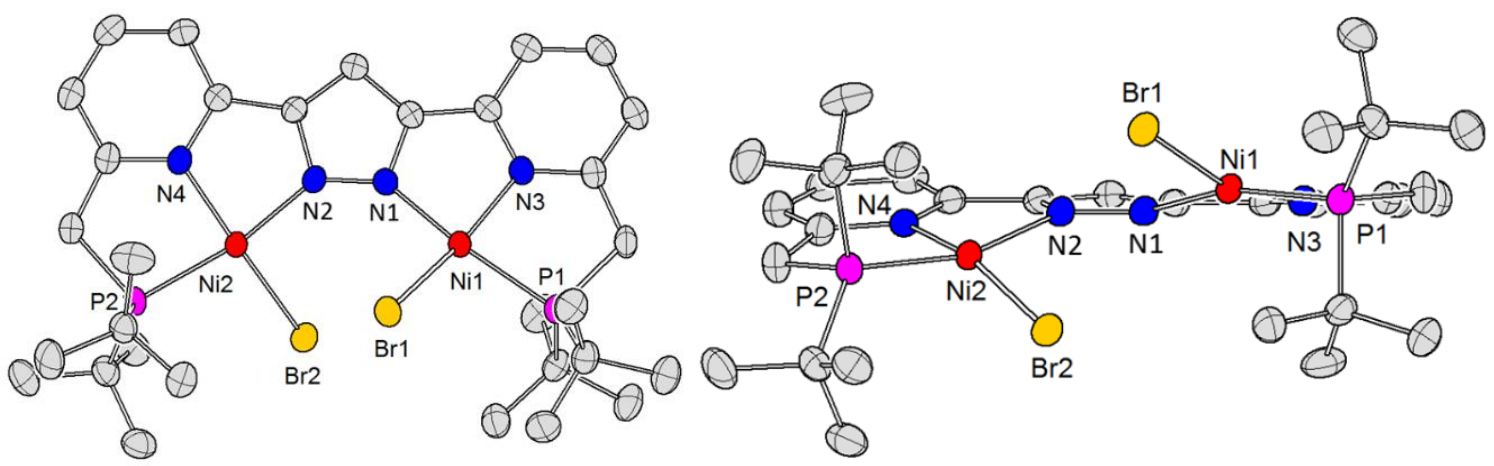

Figure 1.30. Molecular structure (thermal displacement ellipsoids shown at $50 \%$ probability) of the cation of complex $\mathbf{2}$. Hydrogen atoms and anions have been omitted for clarity. Left: top view of the molecular structure. Right: front view of the molecular structure

\begin{tabular}{cc|cc}
\hline \multicolumn{2}{l|}{ Bond Lengths around $\mathrm{Ni}(1) / \AA$} & \multicolumn{2}{l}{ Bond Lengths around $\mathrm{Ni}(2) / \AA$} \\
\hline & & & \\
\hline $\mathrm{Ni}(1)-\mathrm{Br}(1)$ & $2.2869(5)$ & $\mathrm{Ni}(2)-\mathrm{Br}(2)$ & $2.2870(5)$ \\
\hline $\mathrm{Ni}(1)-\mathrm{N}(3)$ & $1.899(3)$ & $\mathrm{Ni}(2)-\mathrm{N}(4)$ & $1.903(3)$ \\
\hline $\mathrm{Ni}(1)-\mathrm{N}(1)$ & $1.946(3)$ & $\mathrm{Ni}(2)-\mathrm{N}(2)$ & $1.939(3)$ \\
\hline $\mathrm{Ni}(1)-\mathrm{P}(1)$ & $2.1792(9)$ & $\mathrm{Ni}(2)-\mathrm{P}(2)$ & $2.1730(9)$ \\
\hline
\end{tabular}

Table 1.2. Selected bond lengths 
A comparison of the Ni-P bond lengths of the square planar $\mathrm{Ni}$ in complex $\mathbf{2}(2.1792 \AA)$ and the square pyramidal $\mathrm{Ni}$ in complex $1(2.3972 \AA)$ exemplified again the plasticity of the Ni-P bond, which was able to shorten more than $0.218 \AA$ upon lowering the coordination number. Indeed, the phosphorus atom can bind depending on the electronic properties of the metal. The dihedral angle $\mathrm{P}(2)-\mathrm{N}(4)-\mathrm{N}(2)-\mathrm{Br}(2)=24.6^{\circ}$ confirmed the deviation from a perfect square plane, which was probably due to steric hindrance between the two bromide atoms located in the bimetallic cleft. The ligand scaffold was quite flexible, allowing the two $\mathrm{Ni}$ ions to minimize their deviation from square plane by torsion of the ligand backbone. The flexibility of the ligand scaffold will be further discussed in the next chapter notably in Figure 2.12. The torsion of the ligand and thus the geometrical modification induced at the nickel ion could possibly influenced the magnetic properties of the complex. Thus, careful analysis of complex $\mathbf{2}$ was of fundamental importance. According to SQUID measurements, complex $\mathbf{2}$ was diamagnetic in solution as well as in the solid state (Figure 1.31), which allowed full and precise characterization by one- and twodimensional NMR methods.

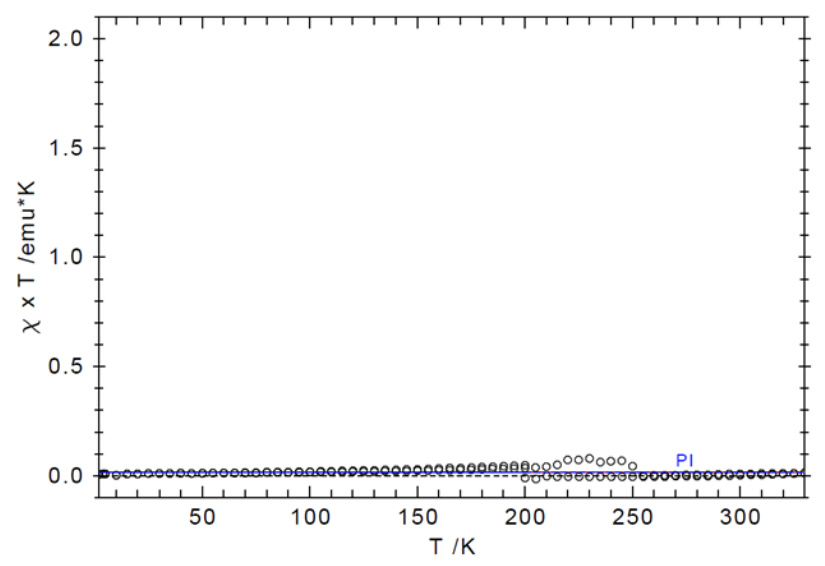

Figure 1.31. Temperature dependence of $\chi_{\mathrm{m}} \mathrm{T}$ of $\left[\mathrm{LH}_{2} \mathrm{Ni}_{2}(\mathrm{Br})_{2}\right] \mathrm{PF}_{6}$ (2) in $\mathrm{CH}_{3} \mathrm{CN}$, showing that the complex is diamagnetic from 4 to $330 \mathrm{~K}$.

Even if the characterization of this complex seemed straightforward, a deeper study in solution revealed delicate information about the dynamic of the complex. As it was seen from the ${ }^{1} \mathrm{H} \mathrm{NMR}$ spectra at different temperatures (Figure 1.32), complex 2 displayed a similar coalescence phenomenon as complex 1 (Figure 1.22). Indeed at $238 \mathrm{~K}$, the two bromides of the bimetallic cleft which were in steric clash, did not flip up and down fast enough in respect to the pyrazolate plane on the timescale of the NMR experiment. Eventually, two inequivalent steric and electronic environments were found above and under the plane. Hence, the diastereotopic protons of the $\mathrm{CH}_{2}$ and the tBu groups had different NMR chemical shifts.

The ${ }^{31} \mathrm{P}$ NMR spectra in $\mathrm{CD}_{2} \mathrm{Cl}_{2}$ revealed a line shape broadening of a single signal at $76.9 \mathrm{ppm}$ depending on the temperature (Figure 1.33). At $25^{\circ} \mathrm{C}$, the signal for the $\mathrm{PF}_{6}{ }^{-}$counter anion (septet at $-144 \mathrm{ppm}$ ) was the only signal observed. When the temperature was decreased, a broad peak at $76.2 \mathrm{ppm}$ was observed and gave a sharp singlet at $208 \mathrm{~K}$. This observation was somehow intriguing as it probably involved a dynamic equilibrium. However, it was questionable that this phenomenon was correlated to the interconversion of the different enantiomers of complex $\mathbf{2}$. It 
was not clear why the signal collapsed in the baseline at room temperature, or why any chemical shift was observed with temperature changes.

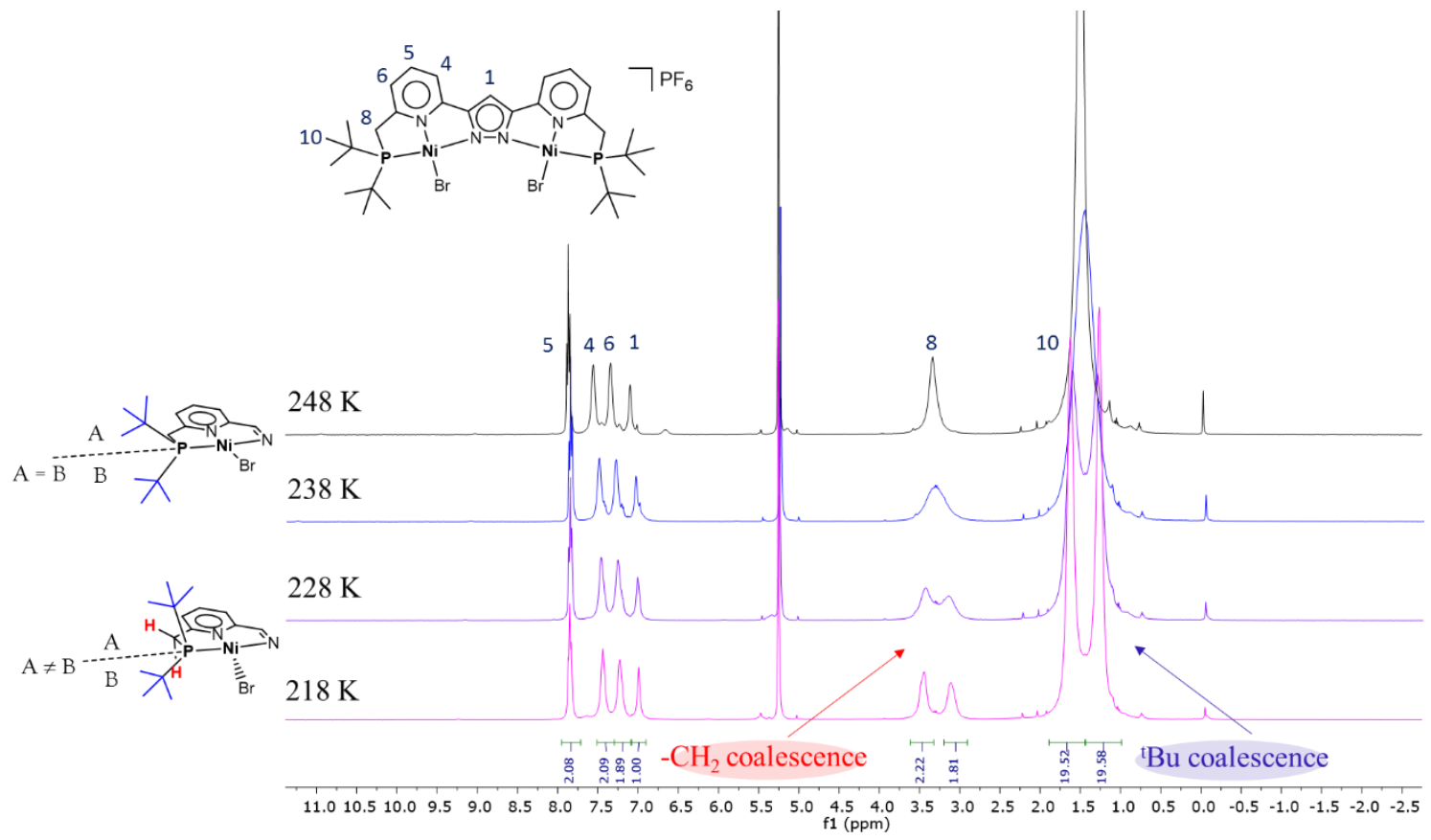

Figure 1.32. ${ }^{1} \mathrm{H}$ NMR (VT, $400 \mathrm{MHz}$ ) spectra of complex 2 in $\mathrm{CD}_{2} \mathrm{Cl}_{2}$.

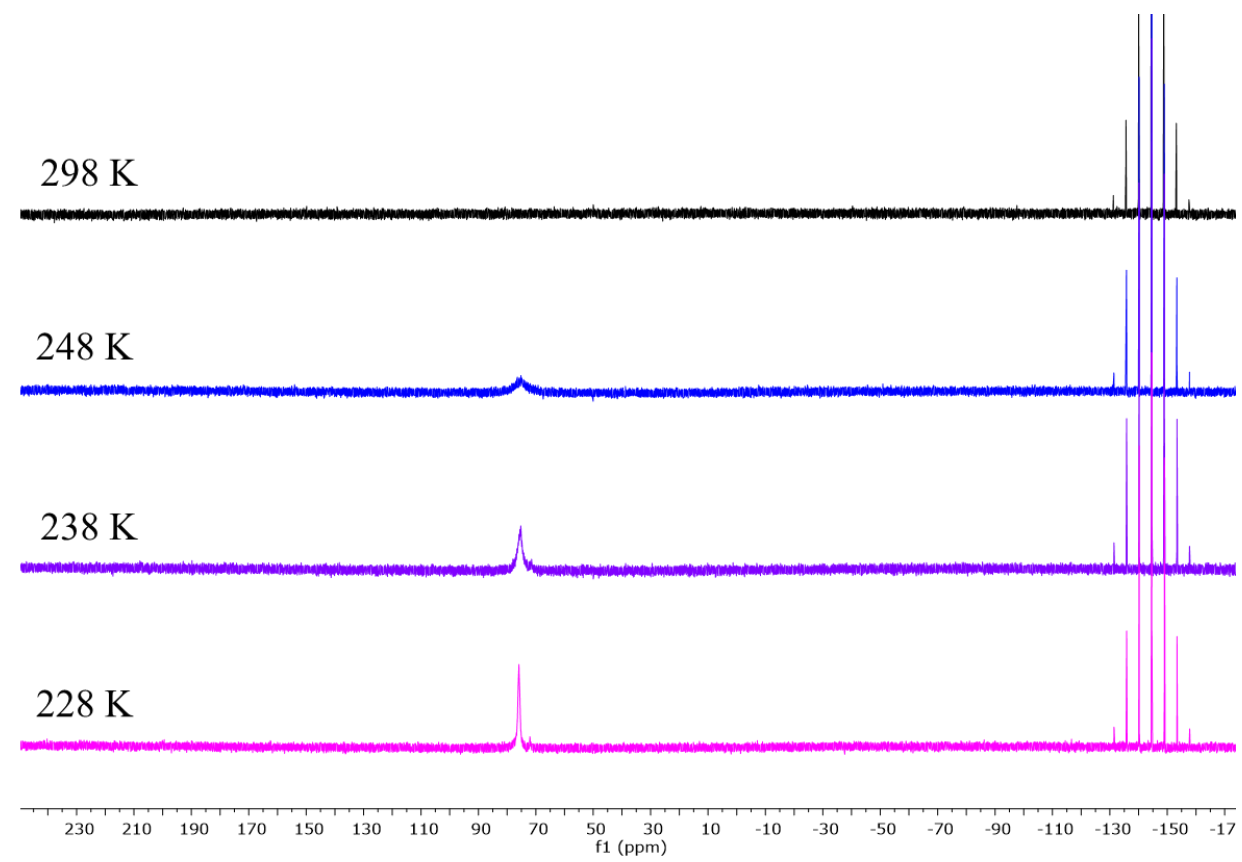

Figure 1.33. ${ }^{31} \mathrm{P}\left\{{ }^{1} \mathrm{H}\right\} \mathrm{NMR}\left(\mathrm{VT}, 162 \mathrm{MHz}\right.$ ) spectra of complex 2 in $\mathrm{CD}_{2} \mathrm{Cl}_{2}$.

UV-vis measurements in $\mathrm{CH}_{2} \mathrm{Cl}_{2}$ at variable temperatures did not reveal significant changes when the temperature of the sample was decreased. At $35^{\circ} \mathrm{C}$, complex $\mathbf{2}$ had an absorption band at $\lambda_{\max }$ $=495.0 \mathrm{~nm}\left(\varepsilon=1065 \mathrm{~mol}^{-1} \cdot \mathrm{L} \cdot \mathrm{cm}^{-1}\right)$ characteristic for $\mathrm{d}-\mathrm{d}$ transitions for $\mathrm{d}^{8}$ square planar 
complexes. Upon lowering the temperature to $-35^{\circ} \mathrm{C}$, a small hypsochromic shift of $\Delta \lambda_{\max }=5$ nm was observed, which was accompanied by an increase in the extinction coefficient: $\varepsilon=1299$ $\mathrm{mol}^{-1} \cdot \mathrm{L} \cdot \mathrm{cm}^{-1}$. While isosbestic points were observed in the visible region, the $\pi \rightarrow \pi^{*}$ transitions of the ligand backbone in the UV region did not display similar isosbestic points. A dissociation equilibrium of the $\mathrm{Ni}-\mathrm{Br}$ bond might be implicated, however experimental observations were not in line with this interpretation: dissolving complex $\mathbf{2}$ in acetonitrile, acetone, dichloromethane or chloroform led to the same red coloured solution with similar NMR chemical shifts. As it will be shown in chapter 2, when the bromide atoms were removed by a salt metathesis with $\mathrm{AgPF}_{6}$ in acetonitrile, free acetonitrile molecules coordinated to the nickel ions and gave a corresponding light orange solution (cf photography in chapter 2). Thus, the $\mathrm{Ni}-\mathrm{Br}$ bond dissociation of complex $\mathbf{2}$ by the solvent was unlikely.

The hypsochromic shift observed for the $\mathrm{d}$-d transitions could be interpreted in terms of the JahnTeller distortion. It was previously demonstrated by NMR that a molecular dynamics was taking place in complex 1 (coalescence shown in Figure 1.22). It was possible that this dynamic was accompanied by slight geometrical modifications at the nickel center, which slightly affected the ligand field splitting parameter $\Delta_{0}$. It was reflected by the changes observed in UV spectroscopy by an energy increase at low temperature of the $d-d$ transitions (Figure 1.34).

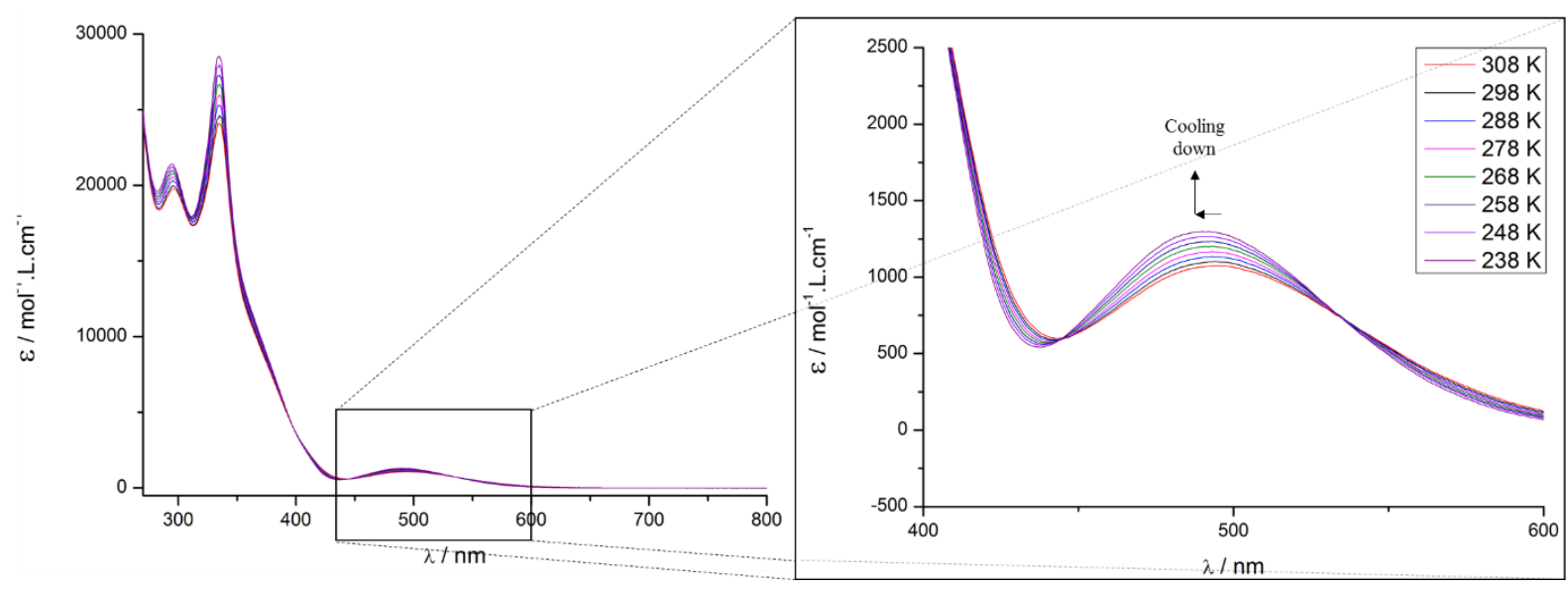

Figure 1.34. UV-vis (VT) spectra of complex 2 in $\mathrm{CH}_{2} \mathrm{Cl}_{2}$

\subsection{Study of the heterobimetallic $\left[\mathrm{LH}_{2} \mathrm{Ni}(\mu-\mathrm{Br}) \mathrm{FeBr}\right] \mathrm{OTf}$ complex}

The existence of the homoleptic Fe complex published by S. Samanta ${ }^{114}$ and the dinickel complexes discussed in sections 1.3 and 1.4 offered interesting perspectives for comparative studies of their reactivity. The challenge of synthesizing a dinuclear complex which combined two different metals is another attractive project that was realised and will be discussed in the following paragraph. 
VIII was dissolved in methylene chloride and one equivalent of $\operatorname{NiDME}(\mathrm{Br})_{2}$ was added. The suspension turned red in several minutes. After one hour of stirring, one equivalent of $\mathrm{Fe}(\mathrm{OTf})_{2}\left(\mathrm{CH}_{3} \mathrm{CN}\right)_{2}$ dissolved in $\mathrm{CH}_{3} \mathrm{CN}$ was added, affording a brown solution. Subsequently, triethylamine was added causing the formation of a yellow precipitate corresponding to $\left(\mathrm{Et}_{3} \mathrm{NH}\right)(\mathrm{OTf})$. It was filtered off, and the remaining solution was set to vapor diffusion with diethyl ether, affording single crystals suitable for X-ray diffraction after three days. Complex $\mathbf{3}$ was particularly sensitive toward oxygen as the colour of a solution of this complex turned from brown to orange under air exposure.

The synthesis of this complex was a delicate exercise which required a detailed inspection of experimental parameters: a) The solubility of VIII in $\mathrm{CH}_{2} \mathrm{Cl}_{2}$ was good while $\mathrm{NiDME}(\mathrm{Br})_{2}$ was insoluble. The amount of $\mathrm{Ni}^{2+}$ in solution being limited and diluted, it favoured the incorporation of a single $\mathrm{Ni}^{2+}$ ion rather than formation of the dinickel species. b) The proton of the pyrazole, which is hydrogen bonded to the pyridine moiety, blocks one coordination site of the ligand. The prototropy is lowered by the low polarity of $\mathrm{CH}_{2} \mathrm{Cl}_{2}$, thus the $\mathrm{N}-\mathrm{H}$ proton is localized and operates as a protecting group, so that the binding of a second $\mathrm{Ni}^{2+}$ is disfavoured prior to addition of a base. Thus, it appeared that dichloromethane was the ideal solvent for the binding of a single $\mathrm{Ni}^{2+}$ ion by the ligand VIII. Once the single binding of $\mathrm{Ni}^{2+}$ ion was achieved, another metal could be added in the presence of a base in order to obtain the heterobimetallic complex.
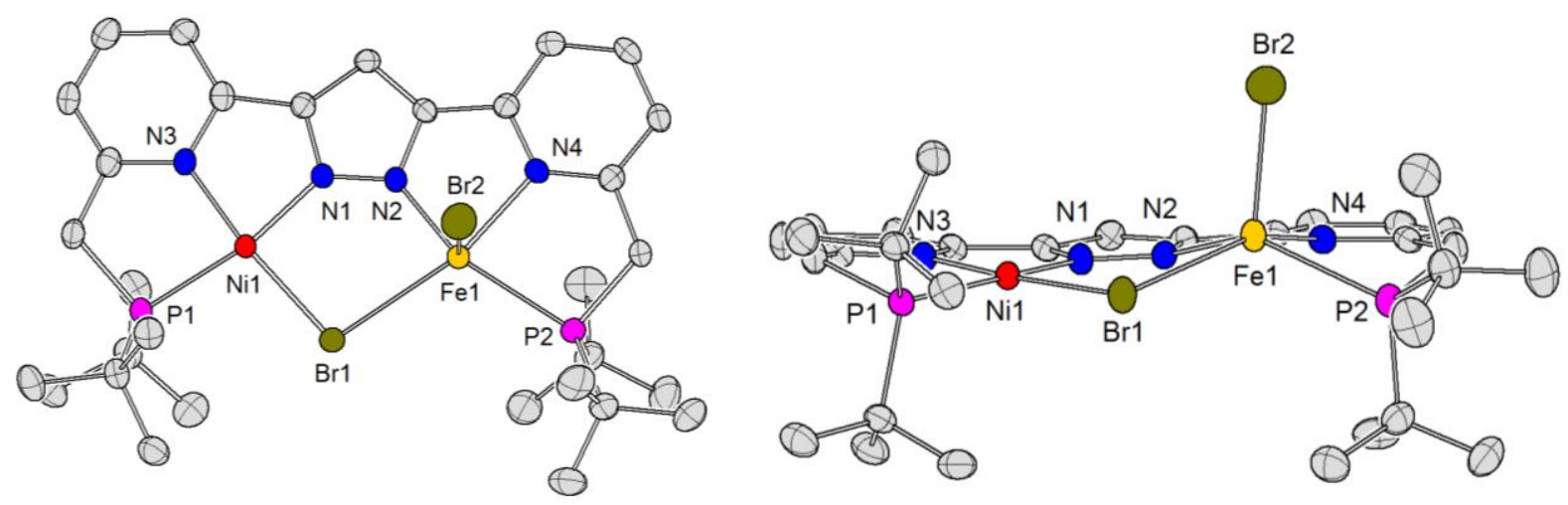

Figure 1.35 Molecular structure (thermal displacement ellipsoids shown at $50 \%$ probability) of the cation of complex $\mathbf{3}$. Hydrogen atoms and anions have been omitted for clarity. Left: top view of the molecular structure. Right: front view of the molecular structure

\begin{tabular}{|c|c|c|c|}
\hline \multicolumn{2}{|c|}{ Bond Lengths around $\mathrm{Ni}(1) / \AA$} & \multicolumn{2}{|c|}{ Bond Lengths around $\mathrm{Fe}(1) / \AA$} \\
\hline $\mathrm{Ni}(1)-\mathrm{N}(1)$ & $1.867(4)$ & $\mathrm{Fe}(1)-\mathrm{N}(2)$ & $2.040(4)$ \\
\hline $\mathrm{Ni}(1)-\mathrm{N}(3)$ & $1.896(4)$ & $\mathrm{Fe}(1)-\mathrm{N}(4)$ & $2.177(4)$ \\
\hline $\mathrm{Ni}(1)-\mathrm{Br}(1)$ & $2.3280(7)$ & $\mathrm{Fe}(1)-\mathrm{Br}(2)$ & $2.3882(9)$ \\
\hline \multirow[t]{2}{*}{$\mathrm{Ni}(1)-\mathrm{P}(1)$} & $2.1906(13)$ & $\mathrm{Fe}(1)-\mathrm{P}(2)$ & $2.4703(14)$ \\
\hline & & $\mathrm{Fe}(1)-\mathrm{Br}(1)$ & $2.7528(8)$ \\
\hline
\end{tabular}

Table 1.3. Selected bond lengths. 
The molecular structure shown in Figure 1.35 revealed a nickel ion with a square planar geometry and an iron ion with a square pyramidal geometry. Contrary to the homoleptic dinickel complex $\mathbf{2}$, the two metals in $\left[\mathrm{LH}_{2} \mathrm{Ni}(\boldsymbol{\mu}-\mathrm{Br}) \mathrm{FeBr}\right] \mathrm{OTf}$ shared a bridging bromide. The distance between the equatorial bromide and the iron was about $2.753 \AA$ which was $0.283 \AA$ longer than the apical bromide. Such distances suggested that $\mathrm{Br}(1)$ was weakly coordinated to Fe. Comparison of $\mathrm{Ni}$ Br bond lengths between complex $\mathbf{2}(2.287 \AA)$ and complex $\mathbf{3}(2.328 \AA)$ gave a hint about the flexibility of the bridging bromide which varied from $0.041 \AA$. A Mössbauer spectrum of complex 3 in the solid state was recorded at $80 \mathrm{~K}$ (Figure 1.36) and displayed a single doublet with a large isomer shift $\left(\delta \mathrm{Fe}=0.90 \mathrm{mms}^{-1}\right)$ and large quadrupole splitting $\left(\Delta \mathrm{E}_{\mathrm{Q}}=3.79 \mathrm{mms}^{-1}\right)$, which suggested the presence of a high spin $\mathrm{Fe}^{2+}(\mathrm{S}=2)$ ion. SQUID data of complex 3 in solution were complicated to interpret and decent fitting was not possible, possibly due to the presence of dynamic processes. An analysis of complex $\mathbf{3}$ in solution was carried out by NMR spectroscopy at variable temperatures.

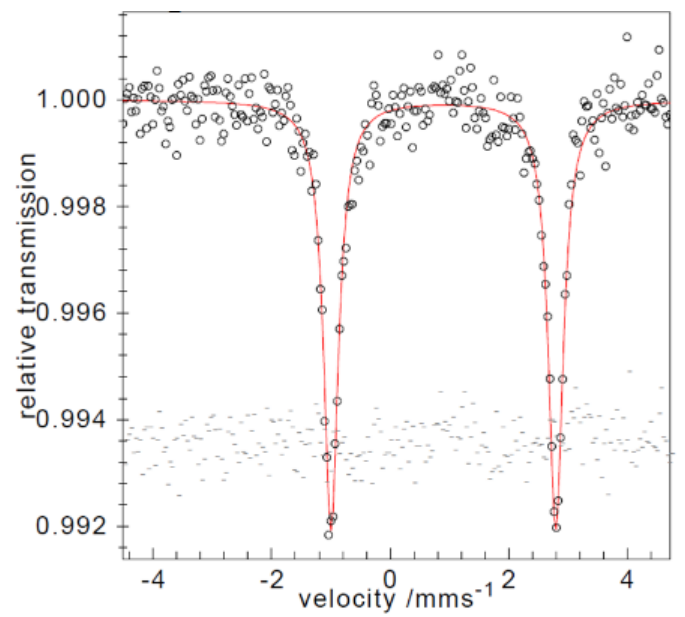

Figure 1.36. Mössbauer spectrum of complex 3 in solid state at $80 \mathrm{~K}: \delta \mathrm{Fe}=0.90 \mathrm{mms}^{-1} \Delta \mathrm{E}_{\mathrm{Q}}=$ $3.79 \mathrm{mms}^{-1}$.

The ${ }^{31} \mathrm{P}$ NMR spectra did not show any signals. The absence of ${ }^{31} \mathrm{P}$ NMR signals was rationalized by the paramagnetic nature of the metal center. However, ${ }^{1} \mathrm{H}$ NMR spectra displayed paramagnetically shifted signals which followed the Curie law (Figure 1.38). The chemical shifts of ${ }^{1} \mathrm{H}$ NMR signals were proportional to $1 / \mathrm{T}$. However, at $298 \mathrm{~K}$, the ${ }^{1} \mathrm{H}$ NMR signals became too broad for reasonable analysis. The assignment and the interpretation of ${ }^{1} \mathrm{H}$ NMR resonances were complicated as 2D NMR spectra did not show any correlation peaks. The presence of more than six NMR signals indicated chemically and magnetically inequivalent halves of the complex. Two larges ${ }^{1} \mathrm{H}$ NMR signals located at $12.7 \mathrm{ppm}$ and $3.1 \mathrm{ppm}$ probably corresponded to the resonances of the two inequivalent $\mathrm{tBu}$ groups of the complex (Figure 1.37). Complex $\mathbf{3}$ had a limited solubility as it was insoluble in dichloromethane, acetone and THF. 


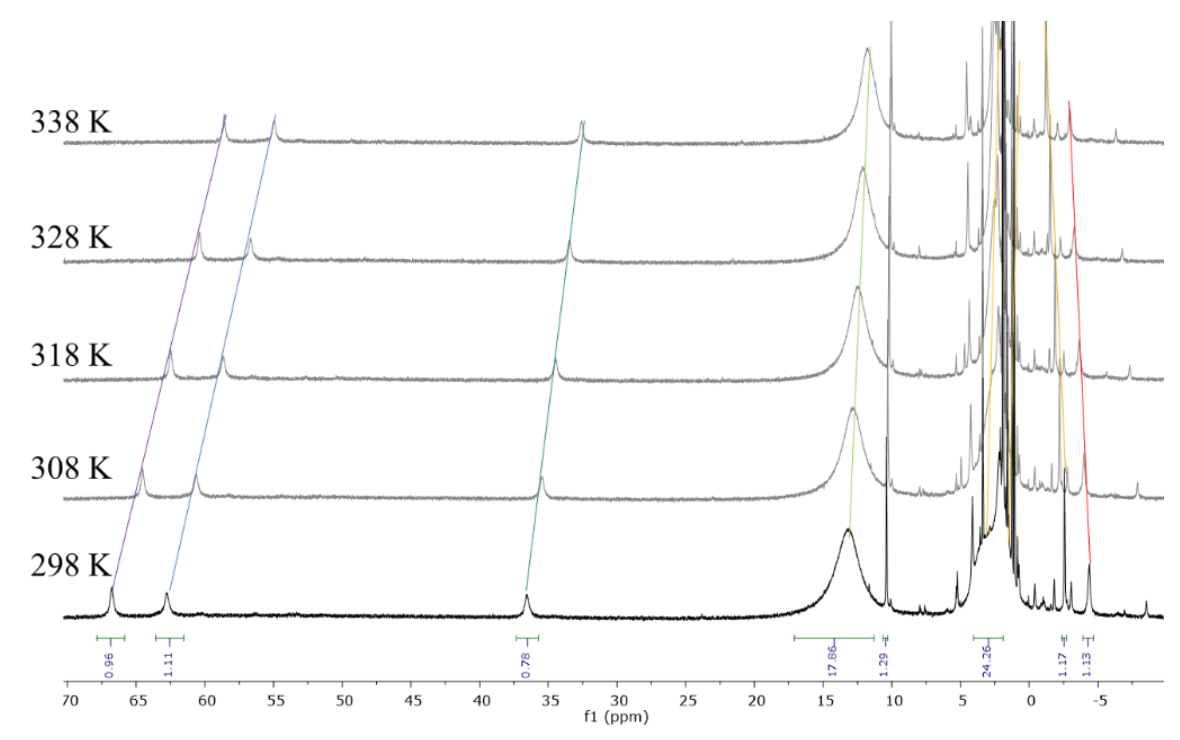

Figure 1.37. ${ }^{1} \mathrm{H}$ NMR spectrum of complex $\mathbf{3}$ in $\mathrm{CD}_{3} \mathrm{CN}$ at different temperatures $(500 \mathrm{MHz})$.

Finally, complex 3 was analysed by UV-vis at different temperatures (cf experimental section) and ESI mass spectrometry (Figure 1.38). Peaks in the region of $809.2 \mathrm{~m} / \mathrm{z}$ were consistent with the cation $\mathrm{LH}_{2} \mathrm{Ni}(\mu-\mathrm{Br}) \mathrm{FeBr}^{+}$.

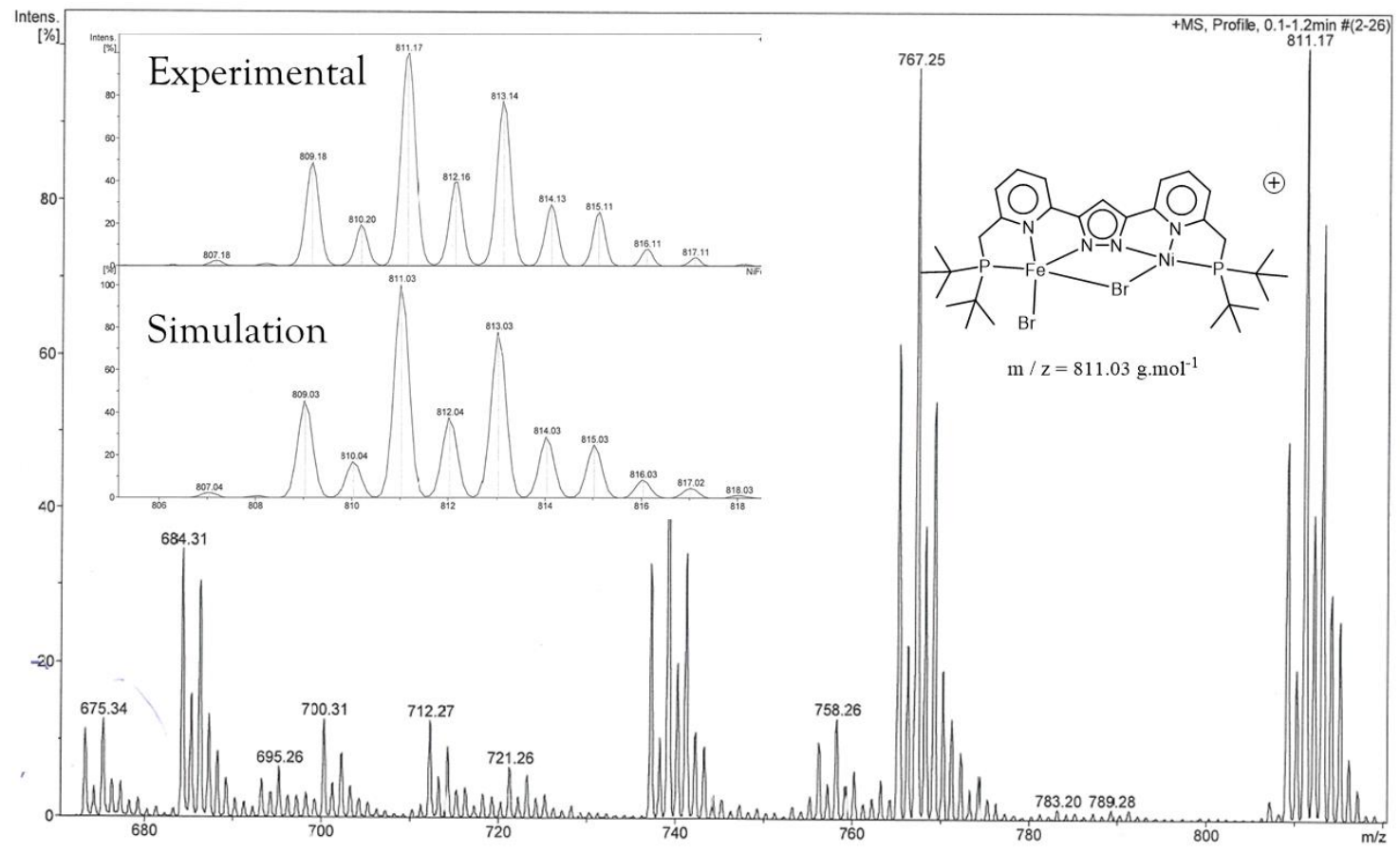

Figure 1.38. ESI mass spectrum of complex 3 in $\mathrm{CH}_{3} \mathrm{CN}$. The inset shows the experimental and simulated isotropic distribution pattern for $\mathrm{LH}_{2} \mathrm{Ni}(\boldsymbol{\mu}-\mathrm{Br}) \mathrm{FeBr}^{+}$. 


\subsection{Conclusion}

In conclusion, ligand VIII was employed for the synthesis of homobimetallic dinickel(II) complexes as well as heterobimetallic nickel(II)/iron(II) complexes in which both metal ions are hosted in pincer-type PNN compartments of the pyrazolate-based binucleating ligand scaffold. Complex 1 was green and paramagnetic while complex $\mathbf{2}$ was red and diamagnetic. As it was demonstrated, $\mathrm{Ni}^{2+}$ might adopt different geometries and spin states depending on the coligand. It was an important prerequisite for the next chapters. Both complexes showed coalescence phenomena in $\mathrm{CH}_{2} \mathrm{Cl}_{2}$ at low temperature. Finally, the synthetic challenge to make a complex of ligand VIII chelating two different metal ions has been achieved within complex $\mathbf{3}$. It represented an interesting system for the study of the synergy between a nickel and an iron ion for substrate transformations. 


\section{Chapter 2: Deprotonation and dearomatization of the ligand scaffold in dinickel(II) complexes}
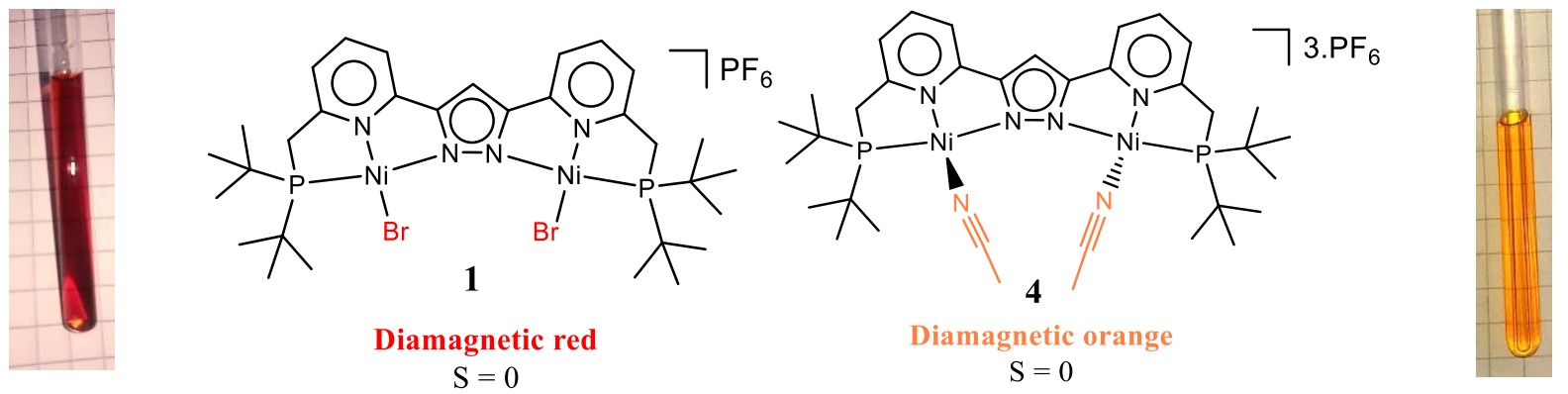

In the previous chapter, the properties of two simple dinickel complexes were described. $\left[\mathrm{LH}_{2} \mathrm{Ni}_{2}(\mathrm{Br})_{2}\right] \mathrm{PF}_{6}(\mathbf{1})$ has the advantage of being diamagnetic, which renders NMR spectroscopy helpful in order to monitor the reactivity of this complex in-situ. In this chapter, the reactivity of $\left[\mathrm{LH}_{2} \mathrm{Ni}_{2}\left(\mathrm{Br}_{2}\right] \mathrm{PF}_{6}\right.$ is investigated by simple changes within the coordination sphere of the metal, among them the deprotonation of the side arm leading to the dearomatization of the pyridine.

\subsection{Introduction: (De)aromatization, state of art}

The variety of synergistic interactions between a metal center and a ligand for the facilitation of a chemical process led to different classifications of cooperating ligands. ${ }^{68}$ The choice of metal and the variability of substituents on the ligand offered the possibility to tune the steric and electronic properties of the metal complex where the substrate transformation was taking place. ${ }^{69,70}$ Good catalysts required robust ligand scaffolds that ideally did not decompose during chemical processes. Avoiding the presence of sensitive organic functions (ketones, aldehydes, alcohols, etc ...) in the ligand was preferable because of potential side reactions that occurred under harsh catalytic conditions (temperature, pressure, $\mathrm{pH}, \ldots$..). ${ }^{27,71,72}$ The longevity of a complex was generally correlated to the non-alterability and the coordination strength of the ligands (denticity, nature of donating atoms, size of chelating rings, etc ...). However, the design of catalysts involving cooperating ligands was a strategy based on reversible chemical alteration of the ligand backbone within its interaction with the metal center. ${ }^{73}$ This was exemplified by its occurrence in metalloenzymes, for example in galactose oxidase. ${ }^{74}$ Some examples of metal-ligand cooperative effects in $\mathrm{Ni}^{2+}$ complexes were mentioned below. 
<smiles>FC(F)(F)C1=C(C(F)(F)F)S[N+]2(S1)SC(C(F)(F)F)=C(C(F)(F)F)S2</smiles>

Redox non-innocence

Relevance: reversible activation of ethylene

A

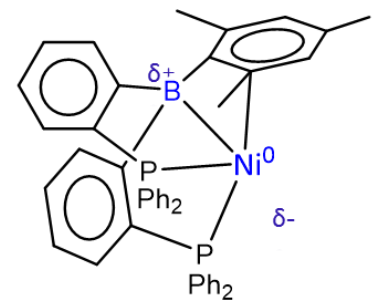

b) Bifunctionality

Relevance: reversible activation of $\mathrm{H}_{2}$

B

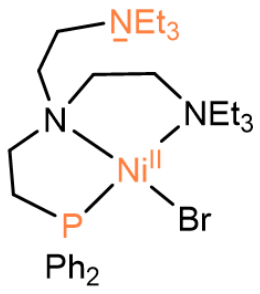

c) Hemilability

Relevance: reversible Spin transition

C

Figure 2.1. Illustration of some metal-ligand cooperating effects useful for physical or chemical transformations. Citation for $\mathbf{A},{ }^{75} \mathbf{B},{ }^{76} \mathbf{C},{ }^{77}$

A case of metal-ligand cooperation was discovered by Milstein in $2005,{ }^{78}$ in which a Ru-PNN complex consisting of a $\left\{\mathrm{R}_{2} \mathrm{P}-\mathrm{CH}_{2}\right\}$ side arm was bound to a pyridine unit. The $\left\{\mathrm{R}_{2} \mathrm{P}-\mathrm{CH}_{2}\right\}$ group was singly deprotonated, whereby the new side arm motif $\left\{\mathrm{R}_{2} \mathrm{P}-\mathrm{CH}\right\}$ was obtained. The pyridine lost its aromatic character whereby the pyridine was so-called dearomatized. Thus, an efficient interplay between an aromatized and a dearomatized form for the development of new catalytic transformations was discovered. ${ }^{78}$ Versatile ligand platforms that displayed such kind of synergy were suitable for any transition metal and rapidly covered a large scope of high yielding catalytic reactions, taking their efficiency out of variety of metal-ligand cooperative effects (Figure 2.2).

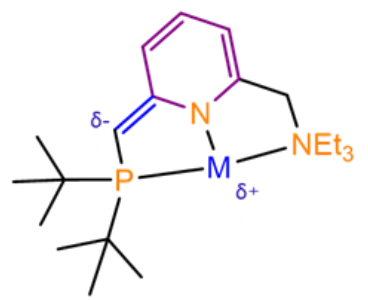
Redox non-innocence
Bifunctionality
Hemilability
$\mathrm{M}=\mathrm{Mn}, \mathrm{Fe}, \mathrm{Co}, \mathrm{Ni}$,
$\mathrm{Mo}, \mathrm{Ru}, \mathrm{Rh}, \mathrm{Pd}$,
Re, Pt

Figure 2.2. One of the pincer complex motifs that enables metal-ligand cooperative effects in homogenous catalysis. See references for the different metals: $\mathrm{Mn},{ }^{79} \mathrm{Fe},{ }^{80} \mathrm{Co},{ }^{81} \mathrm{Ni},{ }^{82} \mathrm{Mo},{ }^{83} \mathrm{Ru},{ }^{78}$ $\mathrm{Rh},{ }^{84} \mathrm{Re},{ }^{85} \mathrm{Pt} .{ }^{86}$

The loss of aromaticity in picoline-based systems was studied in detail. Indeed, the absence of the pyridine aromaticity was evidenced by observing localized $\mathrm{C}=\mathrm{C}$ and $\mathrm{C}-\mathrm{C}$ bonds in the crystallographic data, instead of the average bond length value for delocalized $\mathrm{C}=\mathrm{C}$ double bonds in the six-membered ring (Figure 2.3). Dearomatization of the pyridine was also confirmed by NMR spectroscopy, where the aromatic resonances of the pyridine are typically shifted about $1-2$ ppm upfield. Generally, the circulation of electrons in the aromatic ring induces a magnetic anisotropy which causes the neighbouring protons to be deshielded. Removing the electron circulation (i.e. deprotonation leading to a dearomatized pyridine) was responsible for the upfield shift. 

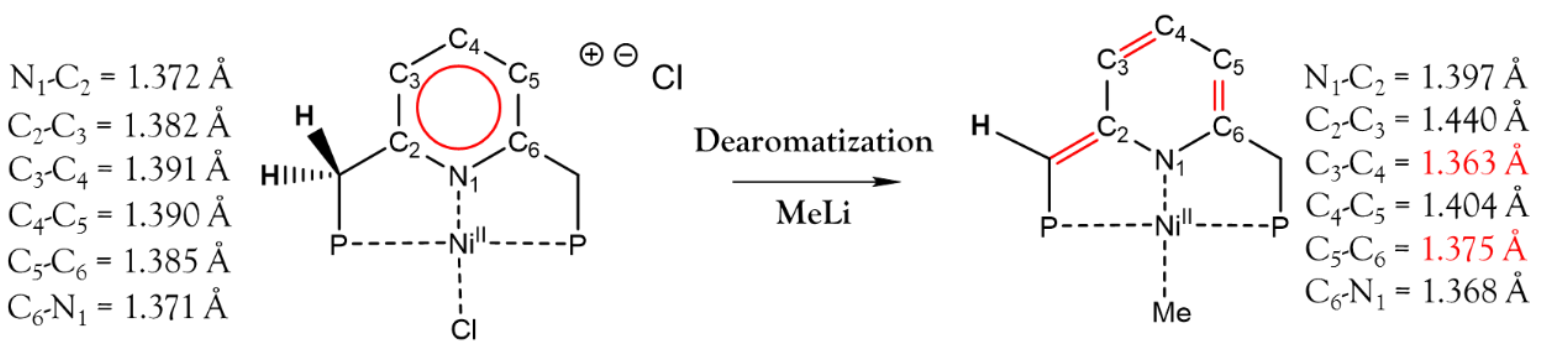

Figure 2.3 Example of dearomatization in a Ni-PNP pincer complex; X-ray data revealed an alternation between $\mathrm{C}=\mathrm{C}$ double and $\mathrm{C}-\mathrm{C}$ single bonds in the pyridine ring. ${ }^{87}$

Such deprotonated complexes were basic entities that could be reprotonated by the addition of protic X-H molecules. The driving force of the reaction was the rearomatization of the pyridine moiety. The apparent simplicity of this acid-base reaction is a suitable approach to achieve polar bond activation in catalysis. ${ }^{88}$ Indeed, the proton of the X-H substrate is transferred to the ligand backbone while the $\mathrm{X}^{-}$coordinates to the metal (Figure 2.4). Milstein's Ru-PNN catalyst demonstrated remarkable versatility toward the activation of water, ${ }^{89}$ primary alcohols, ${ }^{90}$ amines, ${ }^{91}$ dihydrogen ${ }^{92}$ and boronic acids. ${ }^{93}$

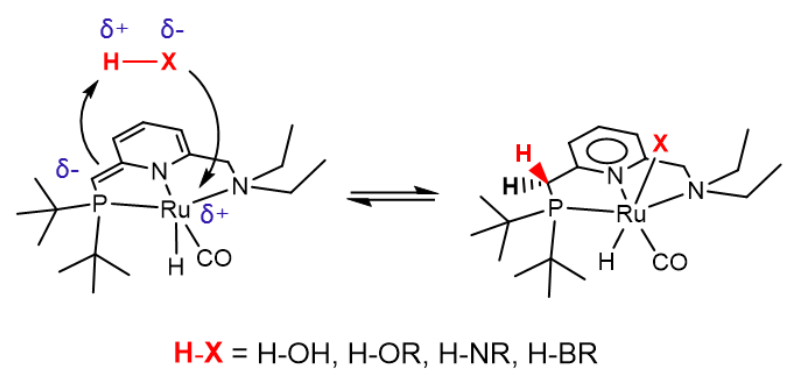

Figure 2.4. Activation of polar bond by Milstein's catalyst

These dearomatized complexes were basic entities, but their unexpected nucleophilicity made them also capable of reversible $\mathrm{C}-\mathrm{C}$ bond formation. Indeed, unsaturated substrates bound reversibly to the complex through $\mathrm{C}-\mathrm{C}$ bond formation on the side arm during a catalytic cycle (Figure 2.5). Milstein's catalyst again remarkably demonstrated its versatility in diverse catalytic reactions involving the hydrogenation of nitriles, ${ }^{94}$ formates, carbonates, carbamates, ${ }^{95}$ and $\mathrm{CO}_{2} \cdot{ }^{96}$ The vast possibilities of reactions unlocked by such performances opened a "new paradigm in bond activation and green catalysis", ${ }^{97}$ and new perspectives for hydrogenation reactions.

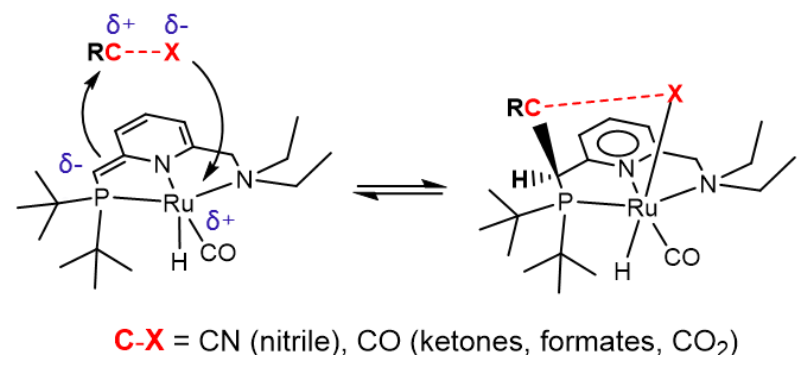

Figure 2.5. Reversible formation of C-C bonds on the side arm of Milstein's catalyst 
Multiple ligands based on protic hydrogen atoms in the $\alpha$-position of the pyridine that trigger the same aromatization-dearomatization feature were developed in the last decade. Interestingly, the concept of dearomatization was extended to other aromatic systems (Figure 2.6).

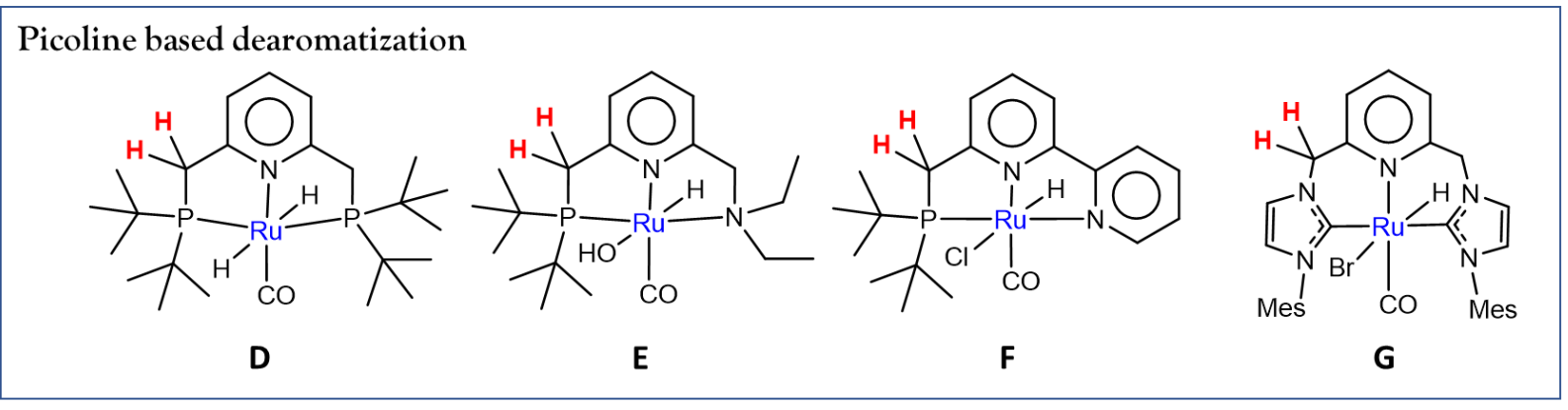

Extended systems suitable for the dearomatization

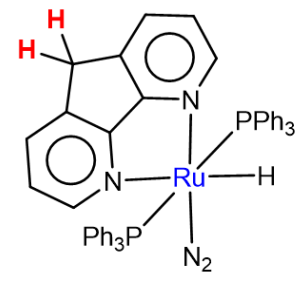

H

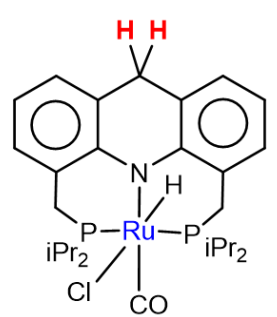

I

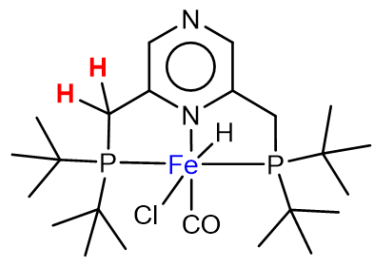

J

Figure 2.6. Selected Ru complexes based on picoline which have acidic $\alpha$-protons (red) that can be deprotonated, with a subsequent dearomatization of the aromatic ring in $\mathbf{D},{ }^{98} \mathbf{E},{ }^{78} \mathbf{F},{ }^{99} \mathbf{G} .{ }^{100}$ Extended ligand systems displaying the same dearomatization phenomenon have been observed in $\mathbf{H},{ }^{101} \mathbf{I},{ }^{102} \mathbf{J} .{ }^{103}$

While the case of the dearomatization in $\mathrm{Ru}$ based complexes was well documented, fewer examples were reported for $\mathrm{Ni}$ complexes. This was due to the scarcity of catalytic applications that derived from the synergetic (de)aromatization cooperative effect in pincer complexes of $\mathrm{Ni}^{2+}$. For example, the dearomatization of the Ni-PNP complex $\mathbf{K}$ (Figure 2.7) was studied in detail by van der Vlugt in 2009. ${ }^{104}$ In the same study, the complex $\mathbf{U}$ represented in Figure 2.10 was able to activate thiols by MLC. Alcohols and water however were not reacting with complex $\mathbf{U}$. The synthesis of a hydride complex by addition of $\mathrm{LiAlH}_{4}$ on complex $\mathbf{N}$ (Figure 2.7) was attempted. An upfield ${ }^{1} \mathrm{H}$ NMR signal at $-18.4 \mathrm{ppm}$ was observed. Milstein later reported in 2013 that the same Ni-PNP complex $\mathbf{K}$ underwent an unusual double deprotonation by addition of three equivalents of MeLi giving complex $\mathbf{L} .{ }^{87}$ The complex $\mathbf{L}$ then reacted with $\mathrm{CO}_{2}$ to give $\mathbf{O}$. The nucleophilic side arm irreversibly formed a $\mathrm{C}-\mathrm{C}$ bound with $\mathrm{CO}_{2}$, which excluded the potential catalytic conversion of $\mathrm{CO}_{2}$ into methanol. A hydrido complex was isolated and structurally characterized by X-ray diffraction in 2018, ${ }^{105}$ which was the one possibly detected in 2009 by van der Vlugt. An alternative way to synthetically access the complex was proposed: an oxidative addition of the $\left\{\mathrm{R}_{2} \mathrm{P}-\mathrm{CH}_{2}\right\}$ side arm upon the reaction of the free ligand with $\mathrm{Ni}^{0}(\mathrm{COD})_{2}$ afforded the hydrido complex $\mathbf{P}$ in quantitative yields. The ligand backbone of this complex involved a deprotonated side arm. The migration of the proton of the side arm $\left\{\mathrm{R}_{2} \mathrm{P}-\mathrm{CH}_{2}\right\}$ to the nickel gave 
a hint about the prospective redox and hydride transfer abilities of this system. The slightly modified complex $\mathbf{P}$ in comparison to $\mathbf{L}$ allowed to reinvestigate the reactivity towards $\mathrm{CO}_{2}$. Nickel complexes displayed attractive reactivities for the reduction of $\mathrm{CO}_{2},{ }^{106,107,108,109}$ and the elaboration of new catalysed reactions. ${ }^{110,111,112}$ The hydrido Ni-PNP complex $\mathbf{P}$ reacted with $\mathrm{CO}_{2}$ and gave an unusual $\mathrm{Ni}-\mathrm{CO}_{2}$ binding motif (complex $\mathbf{R}$ ) reminiscent of the one observed in a $\mathrm{Ni}-\eta^{1}-\mathrm{CO}_{2}-\kappa \mathrm{C}$ complex. ${ }^{113}$ Even though this adduct was the kinetic product, it decomposed into well-defined side products after several hours (complexes $\mathbf{Q}$ and $\mathbf{S}$ ). The promise of such (de)aromatization synergy in this dearomatized $\mathrm{Ni}^{2+}$ complex failed again to find application in catalysis.

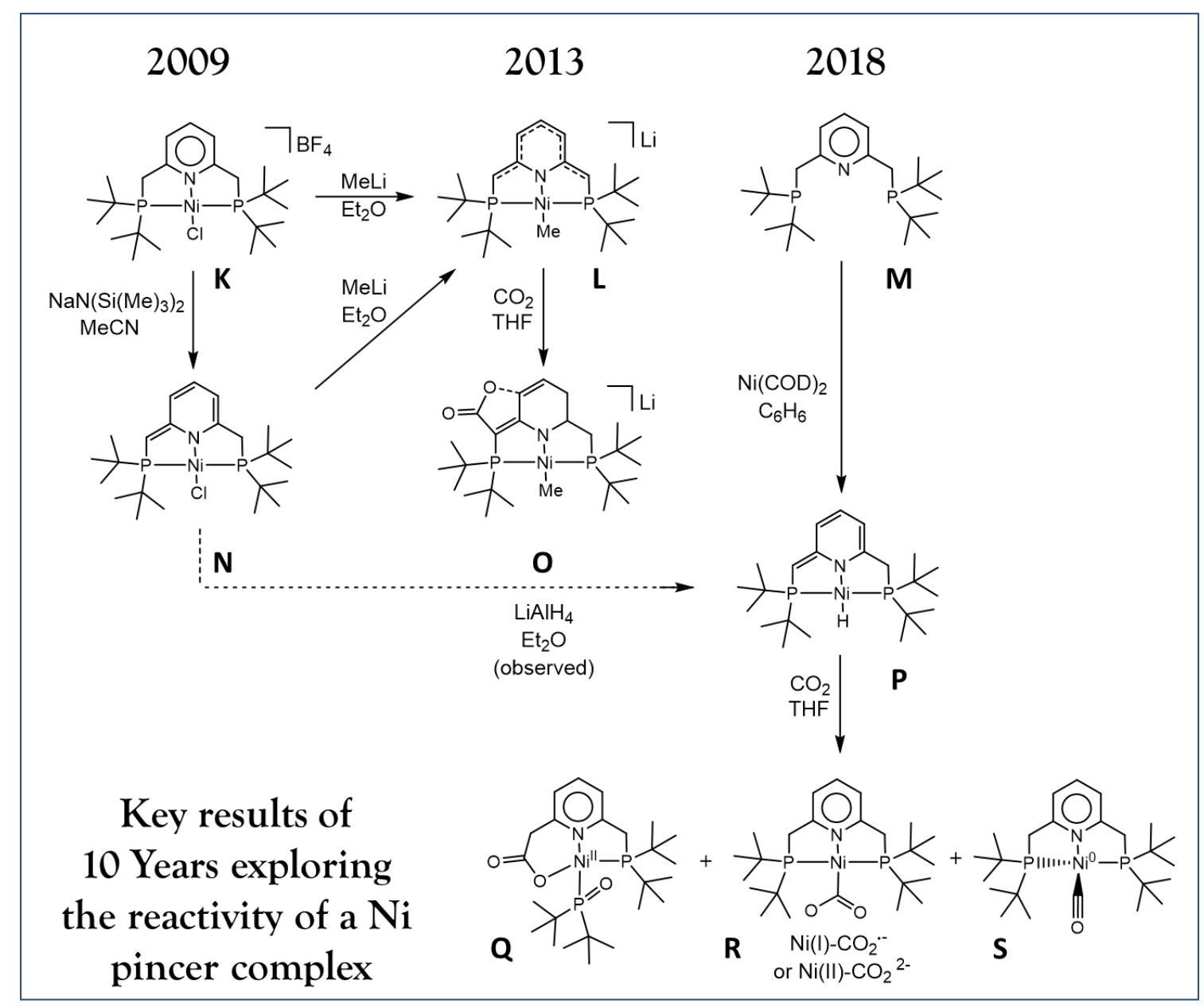

Figure 2.7. Key results of ten years exploring the MLC potential that is involved on a Ni-PNP pincer complex.

Concerning complexes of the Two-In-One pincer ligand, Samanta reported a diiron complex that could undergo a spin transition in solution, ${ }^{114}$ however the (de)aromatization of the ligand was not implicated. Gers-Barlag synthesized and isolated a variety of Two-In-One pincer complexes involving $\mathrm{Zn}$, Co, Rh suitable for (de)aromatization studies (Figure 2.8). In the case of the $\mathrm{Zn}^{2+}$ complex, the addition of a strong base monitored by UV-vis and NMR spectroscopies led to decomposition of the complex over time after a possible dearomatization event. The $\mathrm{Co}^{2+}$ complex was paramagnetic, thus limiting the use of NMR spectroscopy. However, monitoring 
the deprotonation by UV-vis led to the conclusion that dearomatization is not taking place after the addition of a strong base. $\mathrm{Rh}^{1+}$ complexes with various terminal ligands were synthesized, among them $\mathrm{CH}_{3} \mathrm{CN}, \mathrm{SMe}_{2}$ and $\mathrm{CO}$ and were tested in presence of a strong base. The $\mathrm{Rh}^{1+}-\mathrm{CH}_{3} \mathrm{CN}$ and the $\mathrm{Rh}^{1+}-\mathrm{SMe}_{2}$ showed decomposition or partial reactivity which complicated the interpretation of the results. Eventually, the diamagnetic $\mathrm{Rh}^{1+}-\mathrm{CO}$ complex $\mathbf{W}$ in Figure 2.8 was found to undergo double deprotonation with $\mathrm{KOtBu}$ to give complex $\mathbf{X}$. The deprotonation led to dearomatization of pyridines as evidenced by upfield NMR shifts. Unpublished study from A. Gers-Barlag with $\mathrm{Rh}^{1+}$ based dinuclear system ${ }^{115}$ strongly suggested the double dearomatization of the pyridines by the same metrical analysis of the alternating $\mathrm{C}-\mathrm{C}$ and $\mathrm{C}=\mathrm{C}$ bond seen in Figure 2.3. A comparative structural analysis of crystal structures also revealed elongation of the $\mathrm{C} \equiv \mathrm{O}$ bond $(\Delta=0.017 \AA)$ in the $\mathrm{CO}$ terminal ligands and a shortening of the $\mathrm{Rh}-\mathrm{C}$ bond $(\Delta=$ $0.019 \AA$ ) compared to the non-dearomatized complex. The elongation of the $\mathrm{C} \equiv \mathrm{O}$ chemical bond was also observed by IR spectroscopy (Figure 3.9) by a significant decrease of the CO vibrational frequency. Such observations were consistent with an effective $\pi$-backdonation of the metal in the CO ligand after dearomatization of the pyridine.

All Two-In-One pincer complexes involving ligand VIII which were reported to date are represented in Figure 2.8. Among those complexes, only complex $\mathbf{W}$ demonstrated a clear occurrence of deprotonation/dearomatization of the ligand backbone. This chapter will highlight the feasibility of deprotonation reactions on dinickel complex of this kind.

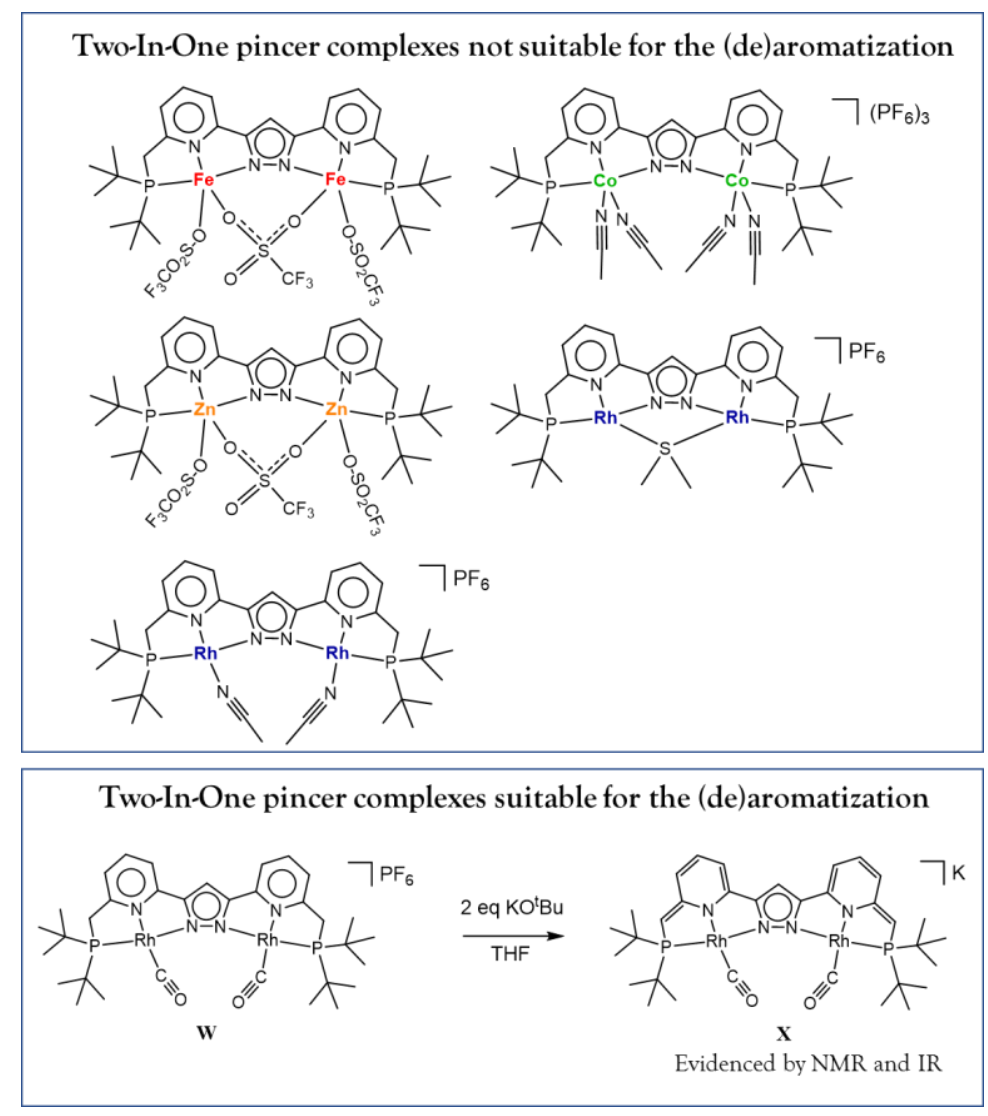

Figure 2.8. Two-In-One complexes that have been tested by Samanta and Gers-Barlag in basic conditions. 


\subsection{Study of the homoleptic complex $\left[\mathrm{LH}_{2} \mathrm{Ni}_{2}\left(\mathrm{CH}_{3} \mathrm{CN}\right)_{2}\right]\left[\mathrm{PF}_{6}\right]_{3}$.}

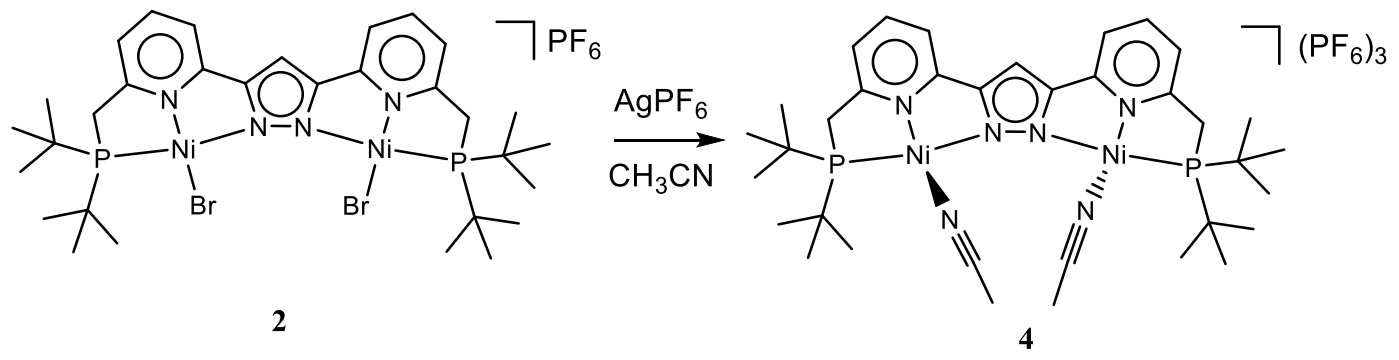

Figure 2.9. Synthesis of $\left[\mathrm{LH}_{2} \mathrm{Ni}_{2}\left(\mathrm{CH}_{3} \mathrm{CN}\right)_{2}\right]\left[3 \mathrm{PF}_{6}\right]$

Following the approach proposed by van der Vlugt with the nickel complexes $\mathbf{T}$ represented in Figure 2.10, ${ }^{104}$ complex $\mathbf{4}$ was specifically synthesized to investigate the deprotonation of the side arm of the complex.

\section{Model of Experimental Approach}

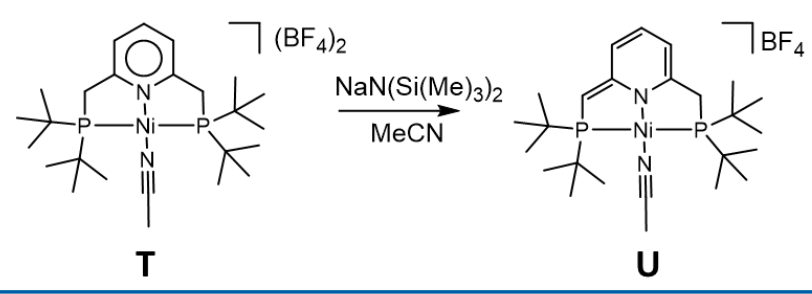

Figure 2.10. Dearomatization of the mononuclear $\mathrm{Ni}^{2+}$ complexes $\mathbf{T}$ involving a terminal acetonitrile ligand. ${ }^{104}$

Complex $\mathbf{4}$ was synthesized by substitution of the bromide ions of complex $\mathbf{2}$ by acetonitrile molecules. A silver (I) salt was required to abstract the bromides of the complex $\mathbf{2}$. $\mathrm{Ni}^{2+}$ complexes tend to display solvatochromic properties: ${ }^{16,117}$ any changes in the coordination sphere of the metal would influence its colour. The colour of complex $\mathbf{2}$ in solution was red and it did not change over time in acetone, acetonitrile, chloroform or dichloromethane. In this regard, the experimental observation were consistent with the fact that the solvents did not favour the $\mathrm{Ni}-\mathrm{Br}$ bond dissociation. Strong donating ligands such as pyridine led to the decomposition of complex $\mathbf{2}$ as a colour change to light yellow was observed. Complex $\mathbf{2}$ was not soluble in protic solvents (water or alcohols) nor in hydrocarbons (toluene, benzene, pentane). Interestingly, if complex $\mathbf{2}$ was dissolved in acetonitrile and if a little of methanol was added, a very slow colour change from red to the similar light orange observed in an NMR sample of complex 4 (photography of chapter 2) occurred in the following weeks.

The synthesis of complex 4 required clean material of complex 2 . It was dissolved in acetonitrile, and two equivalents of $\mathrm{AgPF}_{6}$ were added to the deep red solution. An immediate colour change to light-orange along with a formation of a white precipitate was observed. The solution was filtered and set to vapor diffusion with $\mathrm{Et}_{2} \mathrm{O}$ resulting in the formation of single crystal of 
$\left[\mathrm{LH}_{2} \mathrm{Ni}_{2}\left(\mathrm{CH}_{3} \mathrm{CN}\right)_{2}\right]\left[\mathrm{PF}_{6}\right]_{3}$ suitable for X-ray diffraction. This complex was sensitive to oxygen and decomposed in several hours under exposure to air.
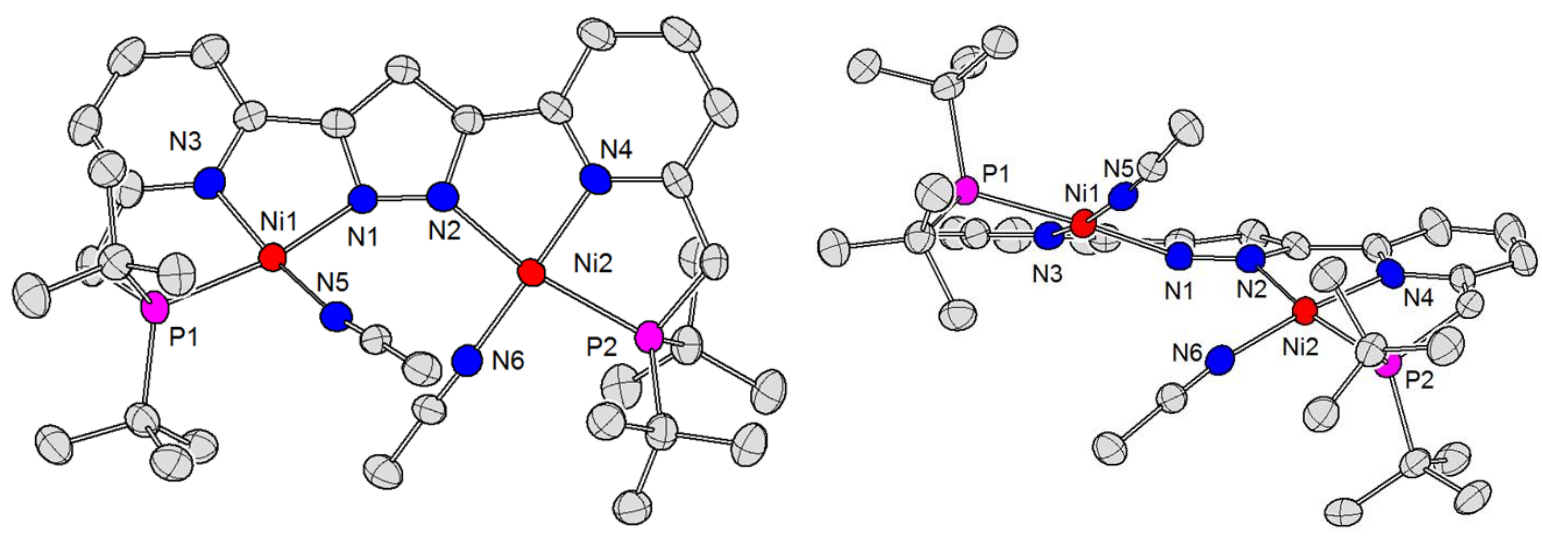

Figure 2.11. Molecular structure (thermal displacement ellipsoids shown at $50 \%$ probability) of the cation of complex $\mathbf{4}$. Hydrogen atoms and anions have been omitted for clarity. Left: top view of the molecular structure. Right: front view of the molecular structure

\begin{tabular}{cc|cc}
\hline \multicolumn{2}{l|}{ Bond Lengths around $\mathrm{Ni}(1) / \AA$} & Bond Lengths around $\mathrm{Ni}(2) / \AA$ \\
\hline & & & \\
\hline $\mathrm{Ni}(1)-\mathrm{N}(5)$ & $1.849(7)$ & $\mathrm{Ni}(2)-\mathrm{N}(6)$ & $1.869(7)$ \\
\hline $\mathrm{Ni}(1)-\mathrm{N}(3)$ & $1.880(6)$ & $\mathrm{Ni}(2)-\mathrm{N}(4)$ & $1.882(6)$ \\
\hline $\mathrm{Ni}(1)-\mathrm{N}(1)$ & $1.937(6)$ & $\mathrm{Ni}(2)-\mathrm{N}(2)$ & $1.932(6)$ \\
\hline $\mathrm{Ni}(1)-\mathrm{P}(1)$ & $2.198(2)$ & $\mathrm{Ni}(2)-\mathrm{P}(2)$ & $2.211(2)$ \\
\hline
\end{tabular}

Table 2.1. Selected bond lengths.

Before studying the dearomatization of complexes, metrical information was extracted from the X-ray crystallographic structures for the understanding of the flexibility of the Two-In-One pincer system. The removal of the two bromido ligands in complex $\mathbf{2}$ led to the formation of complex $\mathbf{4}$ which involved two similar square planar nickel(II). Structural parameters however are largely different between complexes $\mathbf{2}$ and $\mathbf{4}$. The bond length and dihedral angles helped to evaluate the balance between steric and electronic factors that are occurring in these Two-In-One pincer complexes. In Figure 2.12, two structural parameters were studied for the three complexes 2, 4, and $\mathbf{7}$ (the complex $\mathbf{7}$ was not introduced before this discussion but will be studied in detail in the next chapter).

The first parameter was the dihedral angle $(\mathbf{N i}-\mathbf{N}-\mathbf{N}-\mathbf{N i})$ between the two nitrogen atoms of the pyrazolate moiety and the two nickel atoms (Figure 2.12). This parameter gave an idea about the overall torsion of the ligand backbone within the complex. Indeed, the ligand backbone in complex $\mathbf{4}$ was twisted and a dihedral angle of $63.9^{\circ}$ was found. In complex $\mathbf{2}$, the ligand backbone appeared less twisted and the dihedral angle was smaller $\left(47.3^{\circ}\right)$. Finally, in complex 7 the ligand backbone was planar and a dihedral angle of $1.1^{\circ}$ was found.

The second parameter was the four-coordinate geometry index $\tau_{4}$ calculated for the three complexes. This parameter has been previously used to determine the geometry of for four- 
coordinate copper complexes. ${ }^{53,118}$ It is a value which gives an idea about the deviation from the perfect square planarity $\left(\tau_{4}=0\right)$ of the metal within a complex. The more distorted the square planar complex, the higher the $\tau_{4}$ value. It reaches a maximum value when the complex adopts a tetrahedral geometry $\left(\tau_{4}=1\right)$. In complex 2 , the deviation from square planar geometry was the highest with $\tau_{4}=0.25$. In complex $\mathbf{4}$, the $\tau_{4}$ value was smaller and indicated an increase of the square planar character of the nickel ions $\left(\tau_{4}=0.14\right)$. Finally, in complex $\mathbf{7}$, the nickel ions adopted an almost ideal square planar conformation $\left(\tau_{4}=0.095\right)$.

The analysis of these two parameters indicated that the twist of the ligand backbone was likely correlated to the size of the terminal ligands. Indeed, the ligand scaffold was able to twist in order to prevent the steric clash of the two terminal ligands, for example with the $\mathrm{Br}^{-}$ions in complex 2 or with the $\mathrm{CH}_{3} \mathrm{CN}$ ligands in complex 4 . If the size of the terminal ligands was reduced to the minimal size of one proton (respectively a hydride in complex 7), the Two-In-One pincer systems adopted an overall and complete planar conformation. It was noteworthy that the twist of the backbone (increase of the dihedral angle $(\mathbf{N i}-\mathbf{N}-\mathbf{N}-\mathbf{N i})$ ) led to an increase of the intermetallic $\mathrm{Ni} \cdots \mathrm{Ni}$ distance in complexes $\mathbf{2}, \mathbf{4}$ and $\mathbf{7}$.

The comparison between complexes $\mathbf{2}$ and $\mathbf{4}$ was especially interesting. In complex $\mathbf{4}$, the twist of the ligand backbone was the highest (dihedral angle $=63.9^{\circ}$ ), however the $\tau_{4}$ value was lower (improved square planarity of the $\mathrm{Ni}^{2+}$ ions). In complex $\mathbf{2}$, the ligand backbone was less twisted (dihedral angle $=47.3^{\circ}$ ) but the deviation from square planarity of the $\mathrm{Ni}^{2+}$ ions was more important. Thus, it appeared that the complex 4 was taking advantage from the twist of the ligand backbone to improve the square planarity at the $\mathrm{Ni}^{\mathrm{II}}$ ions. Eventually, the twist of the ligand backbone and the $\tau_{4}$ values in complex $\mathbf{2}$ and $\mathbf{4}$ reflected an elusive balance between steric and electronic factors of the terminal ligand coordinated at the $\mathrm{Ni}^{2+}$ ions. The electronic influence of the ligand will be discussed in the following paragraph by mean of UV-vis measurements.

Chapter 1

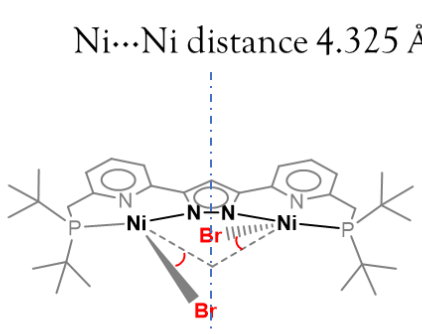

Complex 2
Chapter 2

$\mathrm{Ni}$...Ni distance $4.347 \AA$

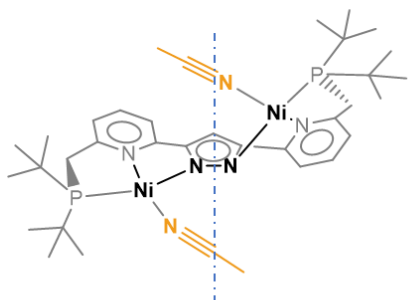

Complex 4

Dihedral angle:

$(\mathrm{Ni}-\mathrm{N}-\mathrm{N}-\mathrm{Ni})$

$=63.9^{\circ}$

$\tau_{4}=0.14$
Chapter 3

$\mathrm{Ni}$...Ni distance $4.156 \AA$

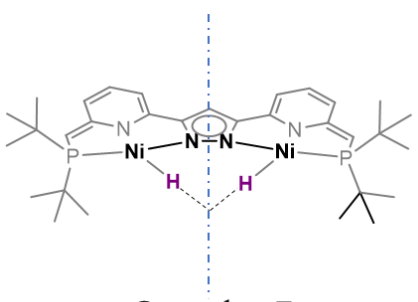

Complex 7

Dihedral angle:

$(\mathbf{N i}-\mathbf{N}-\mathbf{N}-\mathbf{N i})$

$=1.1^{\circ}$

$\tau_{4}=0.095$

$\begin{aligned} & \text { Deviation from } \\ & \text { square planarity: }\end{aligned} \tau_{4}=0.25$

Figure 2.12. Analysis of the torsion of the ligand backbone (dihedral angles) and the deviation from the ideal square planarity of the $\mathrm{Ni}^{2+}$ ions ( $\tau_{4}$ values) within complexes $\mathbf{2 , 4}$ and $\mathbf{7}$. 
The stronger $\sigma$ donation of the acetonitrile molecules in compound $\mathbf{4}$ compared to the bromide ions in complex 2 was reflected by an hypsochromic shift $\Delta \lambda=47 \mathrm{~nm}$ of the maximum of the d$\mathrm{d}$ absorption bands in the UV-vis spectra. This was consistent with experimental colour change of the substance in solution from red $\left(\lambda_{\max }=495 \mathrm{~nm}\right)$ to orange $\left(\lambda_{\max }=448 \mathrm{~nm}\right)$. At $25^{\circ} \mathrm{C}$, complex 4 has an absorption band at $\lambda_{\max }=448 \mathrm{~nm}$ with an extinction coefficient of $\varepsilon=922 \mathrm{~mol}^{\circ}$ ${ }^{1} \cdot \mathrm{L} \cdot \mathrm{cm}^{-1}$. Upon lowering the temperature from $25^{\circ} \mathrm{C}$ to $-35^{\circ} \mathrm{C}$ a $\Delta \lambda=5 \mathrm{~nm}$ was observed, reaching a new $\lambda_{\max }=443 \mathrm{~nm}$ with an extinction coefficient of $\varepsilon=1016 \mathrm{~mol}^{-1} \cdot \mathrm{L} \cdot \mathrm{cm}^{-1}$ (Figure 2.13). Those changes are similar and in the same order of magnitude than those observed for complex $\mathbf{2}$.

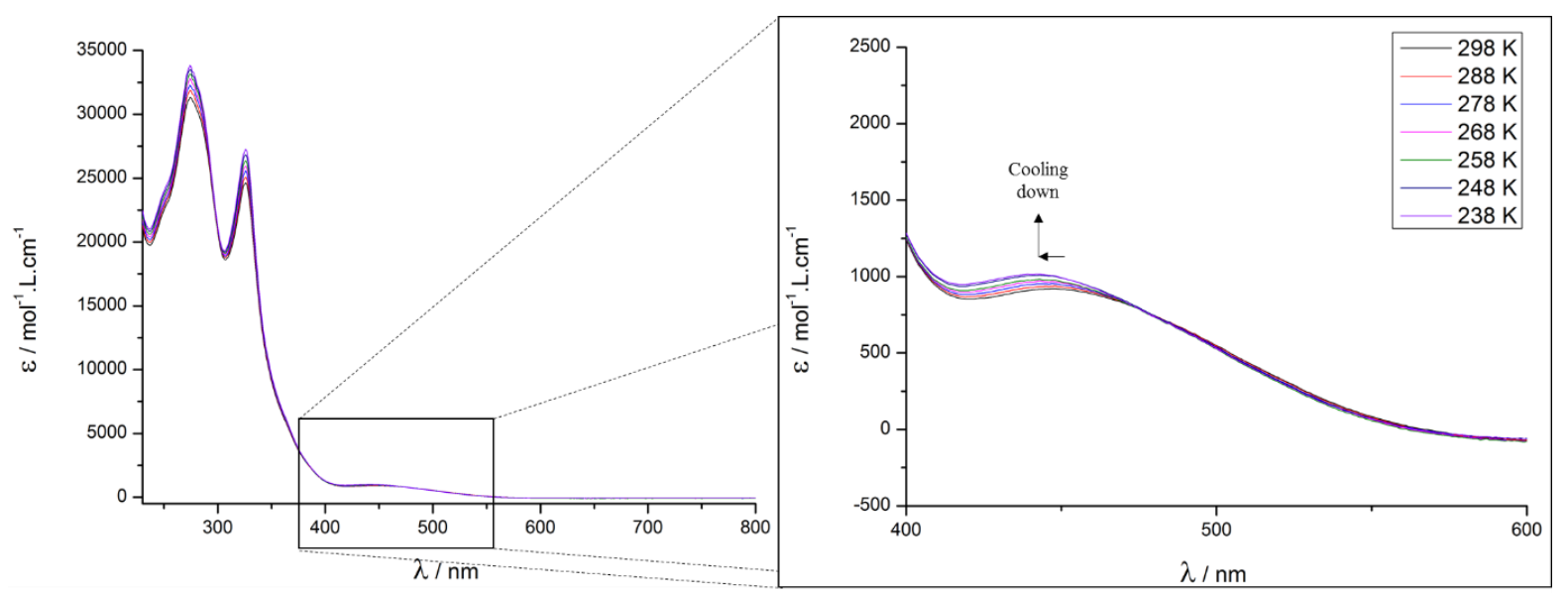

Figure 2.13. UV spectra at variable temperature of $\left[\mathrm{LH}_{2} \mathrm{Ni}_{2}\left(\mathrm{CH}_{3} \mathrm{CN}\right)_{2}\right]\left[3 \mathrm{PF}_{6}\right]$ in $\mathrm{CH}_{3} \mathrm{CN}$.

Extinction coefficient values were clearly indicative of the presence of a complex with nickel(II) ions in square planar ligand environment in solution. This is critical for the following discussion in which the spin state of the complex is discussed. A sample of crystalline material was studied by NMR spectroscopy. The ${ }^{1} \mathrm{H}$ NMR spectrum displayed diamagnetic shifted signals. However the broadness of the signals was intriguing (Figure 2.14). At lower temperature, all signals shifted downfield and disappeared in the baseline (Figure 2.14). At high temperature, signals were sharp, and the calculated integral matched perfectly with the protons involved in the complex.

The ${ }^{31} \mathrm{P}$ NMR spectrum of complex 4 only showed a signal at $-144 \mathrm{ppm}$ for the $\mathrm{PF}_{6}{ }^{-}$anions. No other phosphorus signals were detected even at different temperatures. In the paramagnetic complex 1, the ${ }^{31} \mathrm{P}$ NMR spectrum (Figure 1.23) revealed a peak at $93.1 \mathrm{ppm}$ at $233 \mathrm{~K}$ which shifted upfield at higher temperature and it was consistent with the Curie law. However, the signal became broader and was difficult to detect at room temperature. The ${ }^{31} \mathrm{P}$ NMR spectrum of the diamagnetic complex 2 (Figure 1.33) revealed a broad signal at $76.2 \mathrm{ppm}$ at $238 \mathrm{~K}$ which vanished in the baseline at room temperature without shift. This phenomenon was likely associated to a dynamic process in solution. Thus, the detection of ${ }^{31} \mathrm{P}$ NMR signals in complex $\mathbf{1}$ and $\mathbf{2}$ was not straight forward. In both cases, the ${ }^{31} \mathrm{P}$ NMR signals of the complexes were detected at low temperatures (around $238 \mathrm{~K}$ ), which might be surprising for complex 1 given its paramagnetic character. Surprisingly, no ${ }^{31} \mathrm{P}$ NMR signals except for the $\mathrm{PF}_{6}{ }^{-}$anion was observed at any temperatures. It possibly indicated the presence of both a paramagnetic contribution and the occurrence of a dynamic process in solution. 


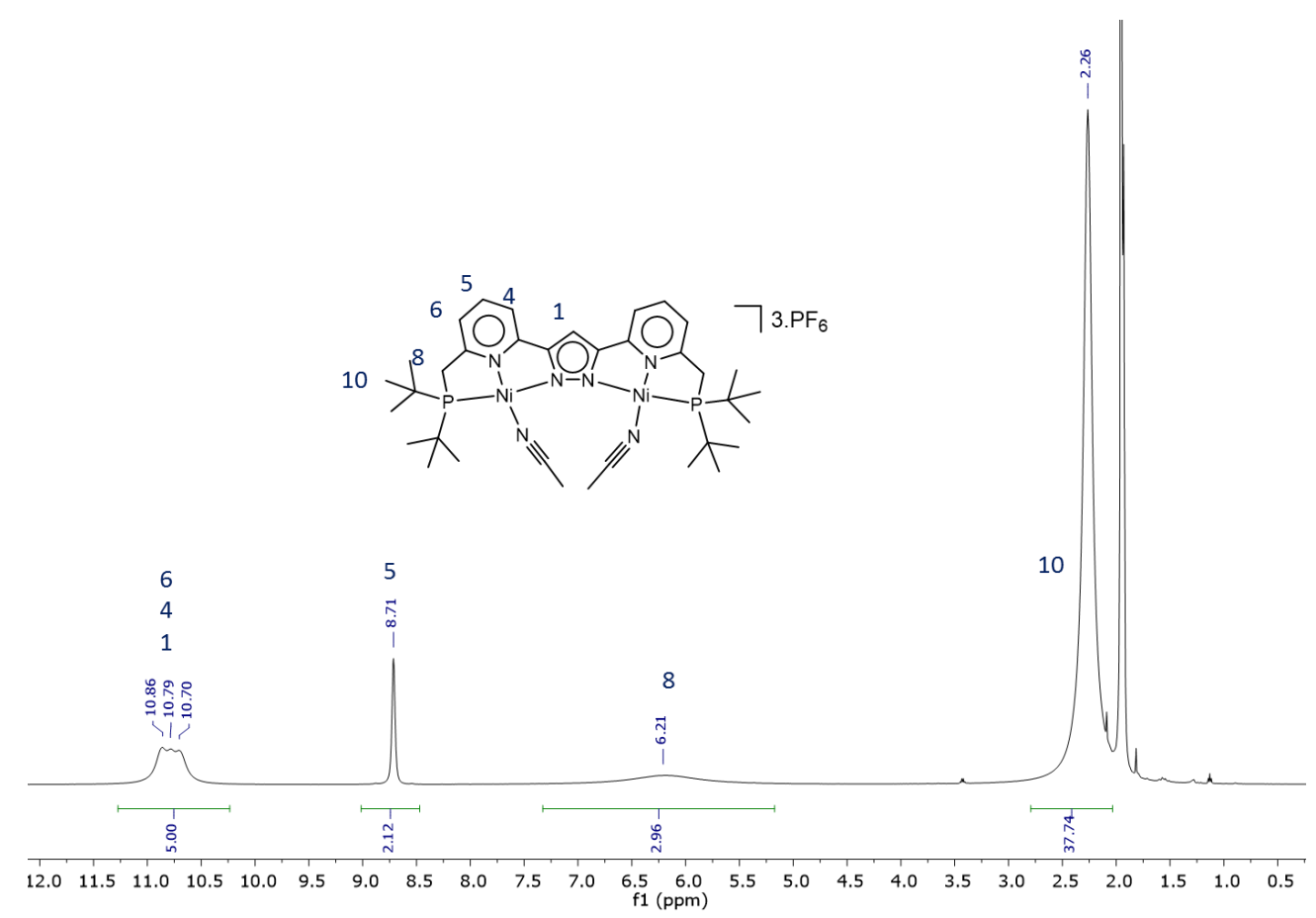

Figure 2.14. ${ }^{1} \mathrm{H}$ NMR spectra of complex 4 in $\mathrm{CD}_{3} \mathrm{CN}(298 \mathrm{~K}), 500 \mathrm{MHz}$.

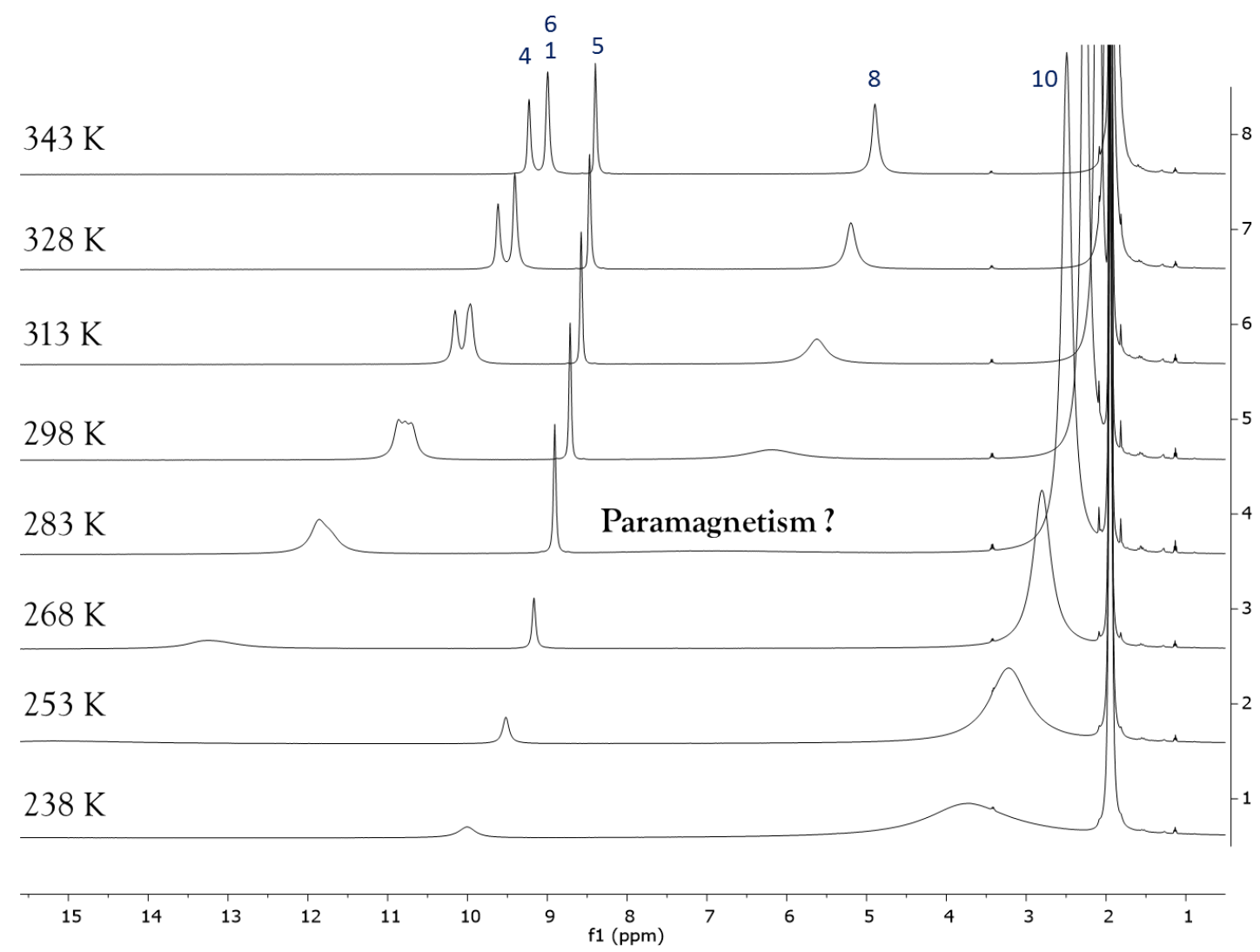

Figure 2.15. ${ }^{1} \mathrm{H}$ NMR spectra of complex 4 at variable temperatures in $\mathrm{CD}_{3} \mathrm{CN}, 500 \mathrm{MHz}$. As can be seen, signals become broader and shift downfield at lower temperatures, which likely indicate the contribution of paramagetism. 


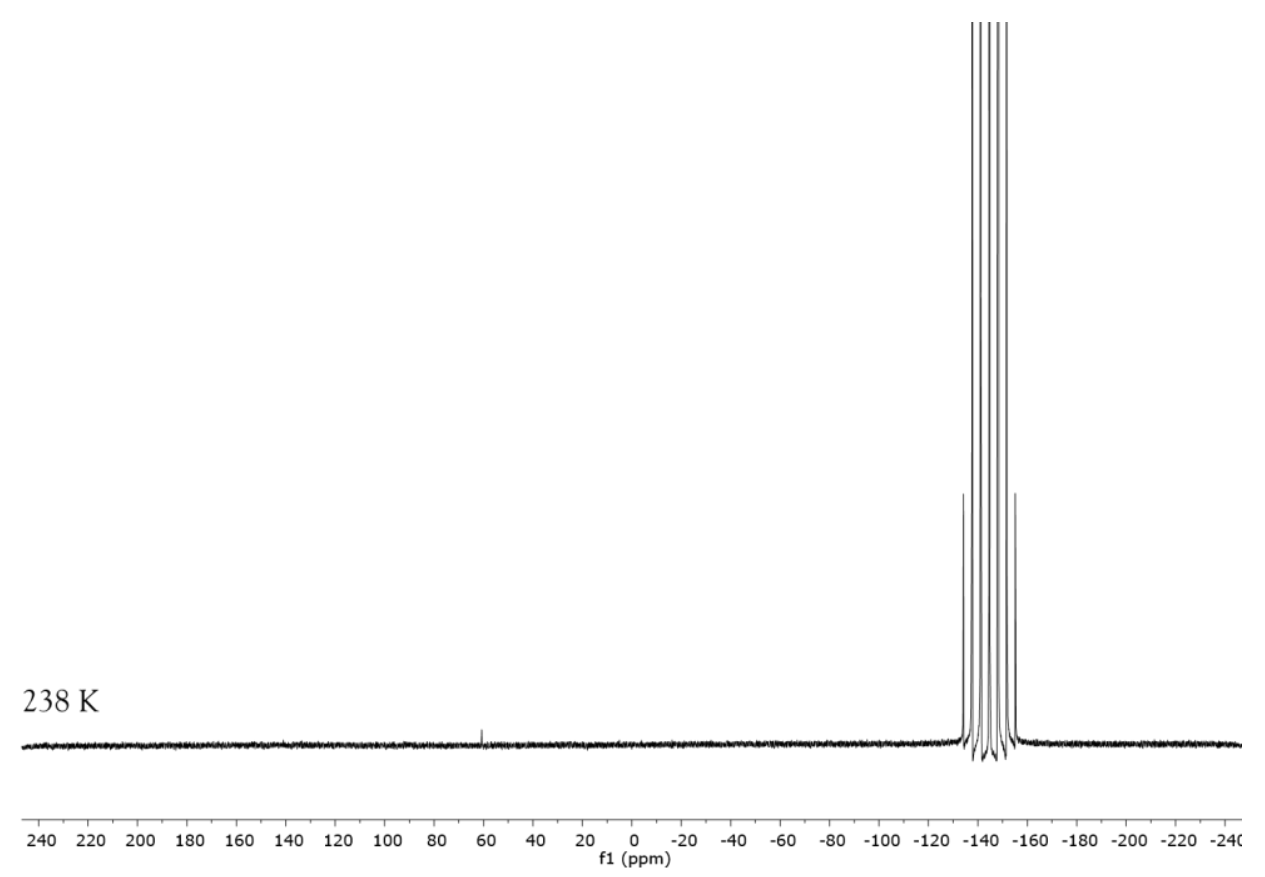

Figure 2.16. ${ }^{31} \mathrm{P}$ NMR spectrum of complex 4 at $238 \mathrm{~K}$ in $\mathrm{CD}_{3} \mathrm{CN}, 500 \mathrm{MHz}$. Only the signal for the $\mathrm{PF}_{6}^{-}$counter ion was observed.

In his thesis ${ }^{58}$ Gers-Barlag reported a similar dinuclear $\mathrm{Rh}^{1+}$ complex which underwent ligand exchange in solution. Analysis of Eyring plots lead to the conclusion that the mechanism involved in the ligand exchange was associative (Figure 2.17). However, the diamagnetic samples used for the study gave no experimental traces of any pentacoordinated paramagnetic intermediate that would form during the reaction. In conclusion, the life-time of the pentacoordinated intermediate was very low.

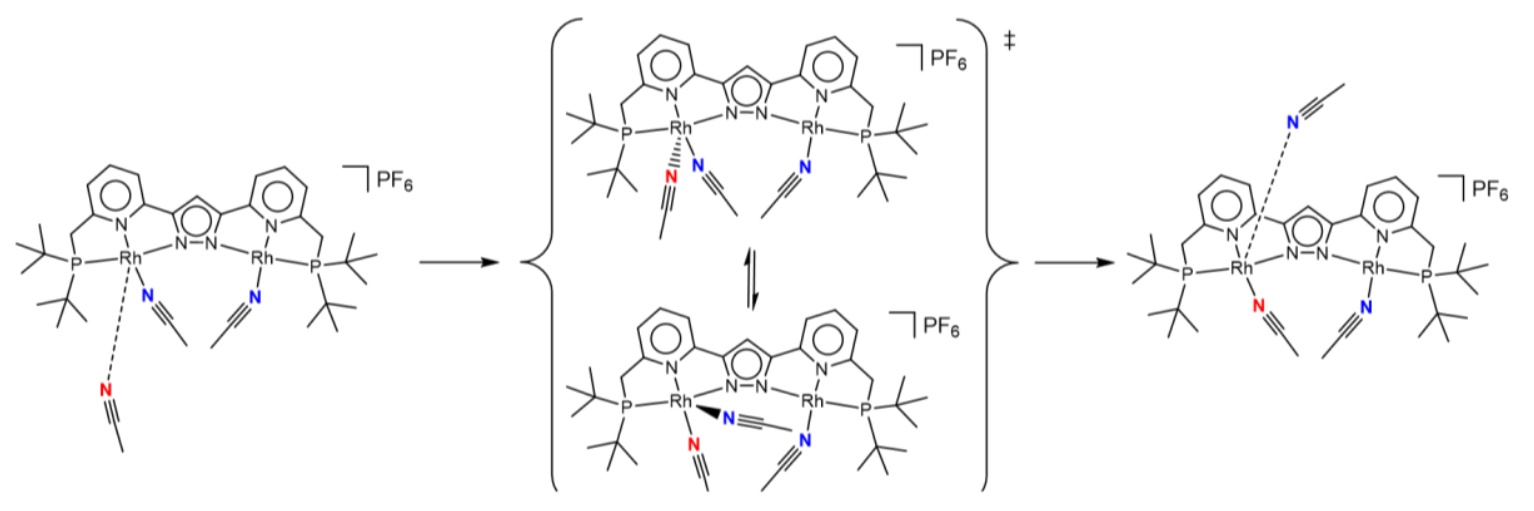

Short living pentacoordinated intermediate

$$
\mathrm{k}_{\text {obs }}(298 \mathrm{~K})=7.6 \times 10^{-2} \mathrm{~min}^{-1}
$$

Figure 2.17. Stated associative-mechanism of ligand exchange by Gers-Barlag.

In contrast to the $\mathrm{Rh}^{1+}$ complex and considering the propensity of $\mathrm{Rh}^{1+}$ to form square planar complexes, the same mechanism of ligand exchange for $\mathrm{Ni}^{2+}$ in complex 4 would involve longerlived pentacoordinated intermediates. These differences could be the reason why a residual paramagnetism has been detected by SQUID before the solution freezes at $238 \mathrm{~K}$ (Figure 2.18) 
and gives paramagnetically shifted resonances in the NMR spectrum at low temperature (Figure 2.15). The geometrical interconversion at low temperature of a square planar low-spin $\mathrm{Ni}^{2+}$ complex with its corresponding pentacoordinated high-spin complex has already been studied (Figure 2.1). ${ }^{77}$

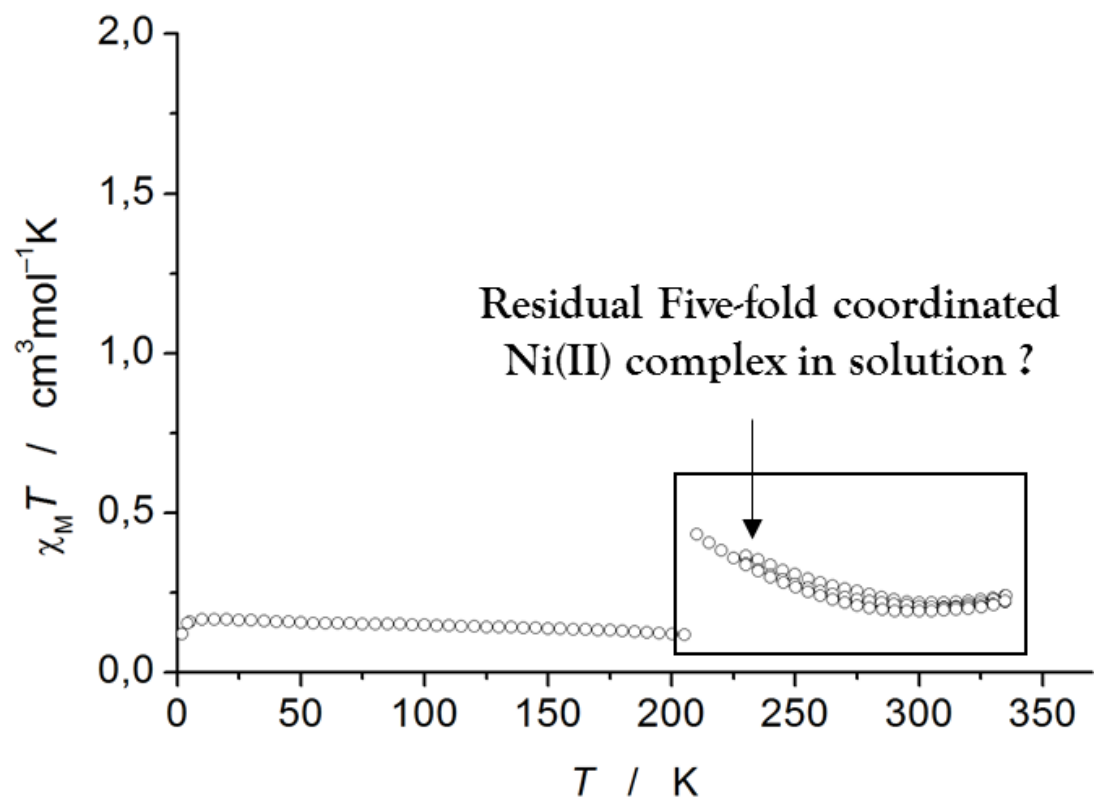

Figure 2.18. Temperature dependence of $\chi_{\mathrm{m}} \mathrm{T}$ of complex 4 in $\mathrm{CH}_{3} \mathrm{CN}$. Ambigous residual paramagnetism of a hypothetical short-lived pentacoordinated $\mathrm{Ni}^{2+}$ complex.

The dearomatization of complex $\mathbf{4}$ was attempted. The investigation of this reaction was limited due the exclusive solubility of the complex in nitriles. When KHMDS (potassium bis(trimethylsilyl)amide) was added to complex $\mathbf{4}$ in acetonitrile, slow colour change from orange to brown was observed. Complicated NMR spectra were obtained, and it was not possible to evidence the dearomatization of the complex.

\subsection{Double dearomatization: NMR and UV investigation}

The mechanism of deprotonation was investigated by Gers-Barlag for the dinuclear $\mathrm{Rh}(\mathrm{I})$ complex W (Figure 2.8). A sequential reaction based on NMR titration experiments was proposed. The sequential reactivity was further confirmed here with the present UV-vis experiment (Figure 2.21). A bright yellow suspension of complex $\mathbf{W}$ in THF was deprotonated with one equivalent of $\mathrm{KO}^{\mathrm{t}} \mathrm{Bu}$ giving a green solution of complex $\mathbf{Y}$ (Figure 2.20). Since the material became soluble after the first deprotonation, the titration of the second step monitored by UV-vis spectroscopy became possible. Portions of $\mathrm{KO}^{\mathrm{t}} \mathrm{Bu}$ were added to a solution of $\mathbf{Y}$ and gave compound $\mathbf{X}$. Three isosbestic points could be observed for this transformation. 


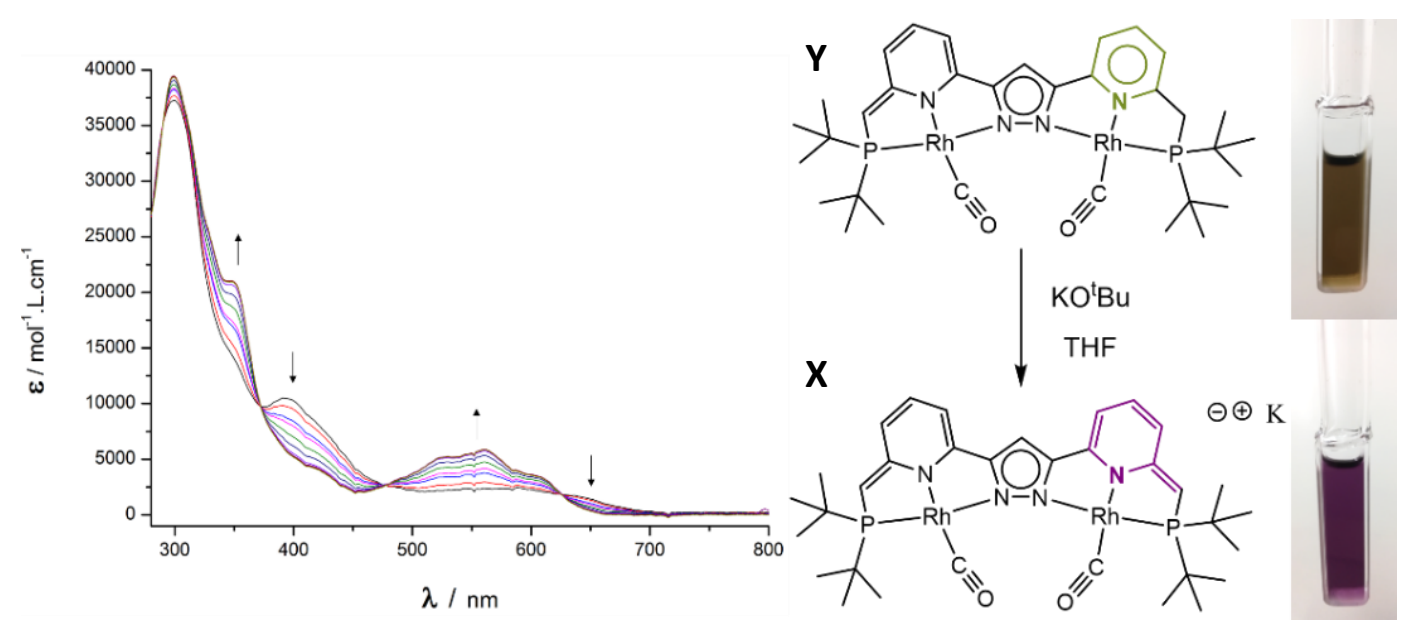

Figure 2.20. Dearomatization of complex $\mathbf{Y}$ to complex $\mathbf{X}$ monitored by a $\mathbf{U V}$-vis titration.

In total, four nickel(II) complexes (1, 2, 3 and 4) of the Two-In-One pincer ligand VIII provided a platform for reactivity studies. Their deprotonation was investigated using $\mathrm{KO} \mathrm{B}^{\mathrm{t}} \mathrm{Bu}$. As discussed previously, complex $\mathbf{4}$ was unsuitable because of solubility limitations. Complexes $\mathbf{1}$ and $\mathbf{3}$ did not show any particular reactivity or expected colour changes. Finally, complexes $\mathbf{2}$ was the only complex that clearly reacted in the presence of a base and confirmed a double dearomatization on the pyridine moieties on the pincer subunits (Figure 2.21).

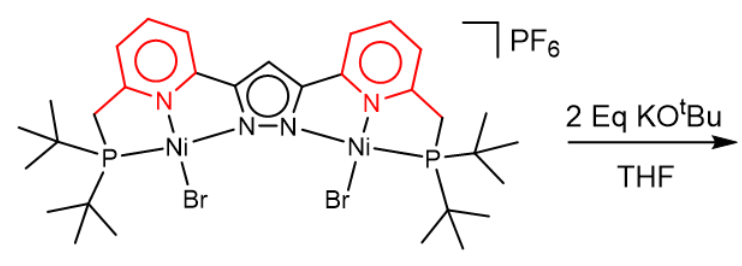

2

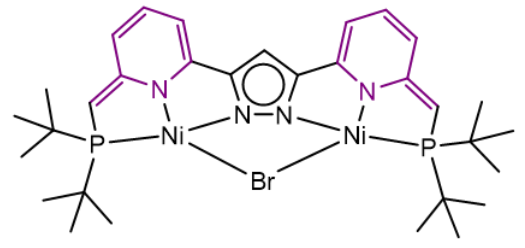

5

Figure 2.21. Double dearomatization of $\left[\mathrm{LNi}_{2}\left(\mathrm{Br}_{2}\right] \mathrm{PF}_{6}\right.$.

$\left[\mathrm{LH}_{2} \mathrm{Ni}_{2}(\mathrm{Br})_{2}\right] \mathrm{PF}_{6}$ (2) was suspended in THF and after addition of $\mathrm{KOtBu}$ (or $\mathrm{KH}$ ), the red suspension turned into a violet solution. The double dearomatization was evidenced by a typical upfield shift of the aromatic protons 4-5-6 of $\left[\mathrm{LH}_{2} \mathrm{Ni}_{2}\left(\mathrm{Br}_{2}\right)_{2} \mathrm{PF}_{6}\right.$ (red box in Figure 2.22) to give the new complex $\left[\mathrm{LNi}_{2}(\boldsymbol{\mu}-\mathrm{Br})\right](\mathbf{5})$ with a new set of aromatic resonances (violet square on Figure 2.22). The integral calculated for the $\left\{\mathrm{R}_{2} \mathrm{P}-\mathrm{CH}_{2}\right\}$ group was equal to 4 in complex 2 . However, the integral value was equal to 2 in complex $\mathbf{5}$ and were in line with the presence of $\left\{\mathrm{R}_{2} \mathrm{P}-\mathrm{CH}\right\}$ groups. Because of the electron enrichment after the deprotonation, all the NMR signals (including ${ }^{1} \mathrm{H},{ }^{31} \mathrm{P}$ and ${ }^{13} \mathrm{C}$ ) were shifted upfield (Figure 2.23). All signals could be assigned by $2 \mathrm{D}$ NMR methods. An unusual long range ${ }^{5} \mathrm{~J}_{\mathrm{P}-\mathrm{H}}=2.37 \mathrm{~Hz}$ between the phosphorus atom and the proton in the para position of the pyridine was detected. The coupling of the hydrogen atom with the phosphorus atom was evidenced by a decoupled ${ }^{1} \mathrm{H}$ NMR spectrum (Figure 2.22 on the right). Multiple attempts to get single crystals led to slow decomposition of the deprotonated complex. This complex was partially soluble in apolar solvent (like hexanes or pentane), which supported the hypothesis that complex $\mathbf{5}$ was neutral. 


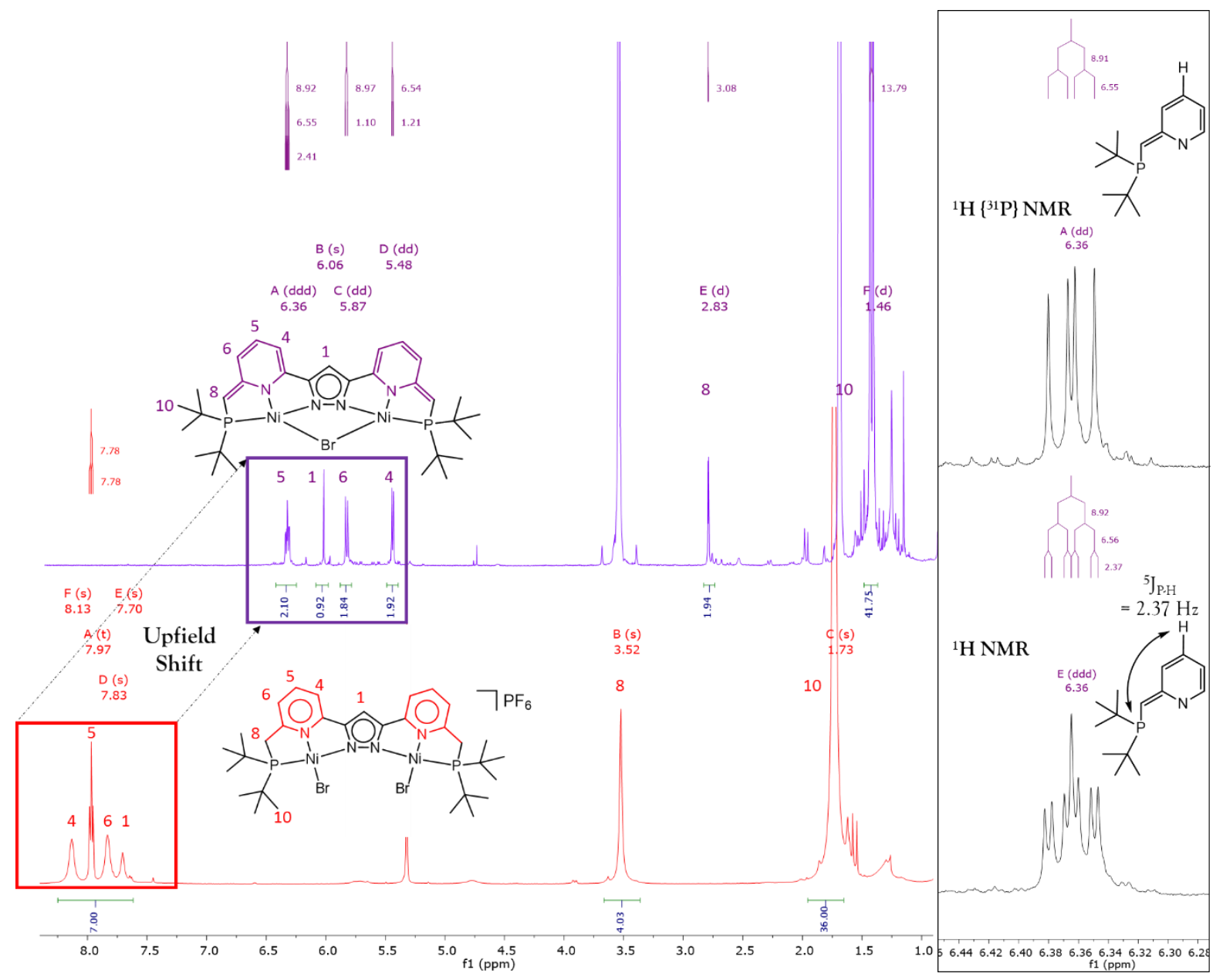

Figure 2.22. ${ }^{1} \mathrm{H}$ NMR $(500 \mathrm{MHz})$ spectra of complex 2 at $298 \mathrm{~K}$ before (red) and after (violet) deprotonation. On the right: A decoupling experiment emphasized a long range ${ }^{5} \mathrm{~J}_{\mathrm{P}-\mathrm{H}}$.

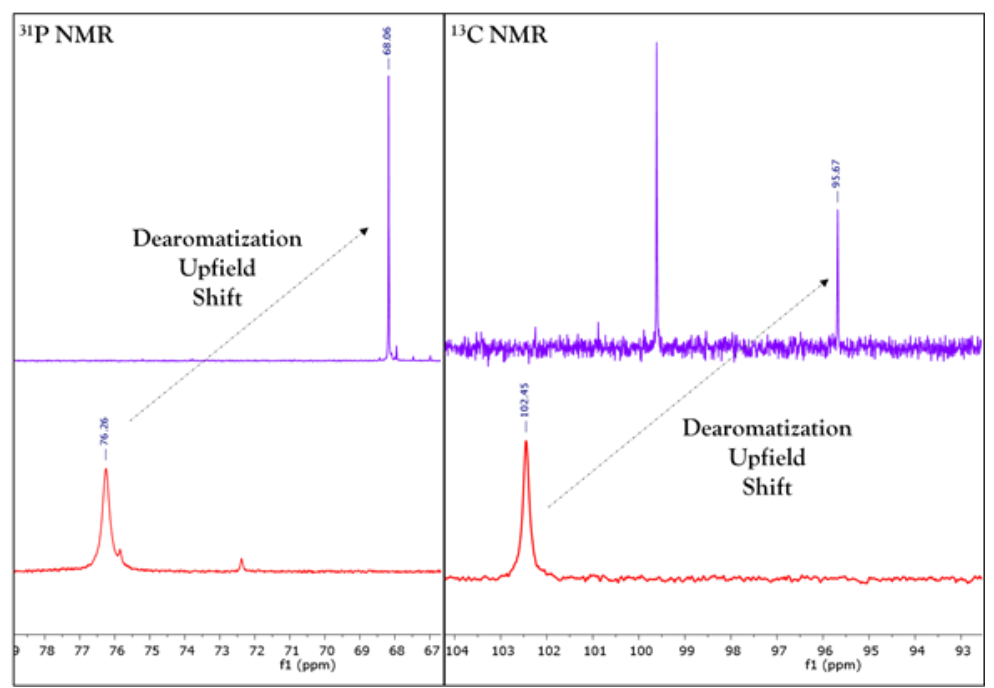

Figure 2.23. Left: ${ }^{31} \mathrm{P}$ NMR spectra and right: ${ }^{13} \mathrm{C}$ NMR spectra (of ${ }^{13} \mathrm{C}-\mathrm{H}$ pyrazole signal) of complex 2 in $\mathrm{CD}_{2} \mathrm{Cl}_{2}$ at $298 \mathrm{~K}$ before (red), and after deprotonation in THF-d (violet). Spectra are truncated for more clarity. 


\subsection{Observation of other dearomatized nickel(II) Two-In-One pincer complexes}

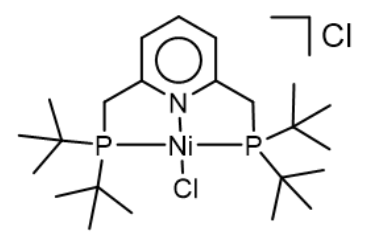

K

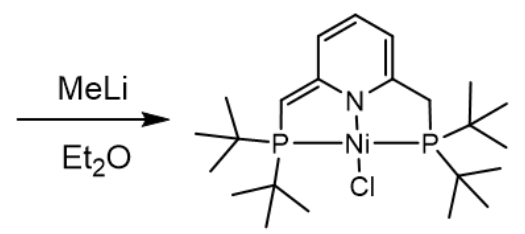

$\mathbf{N}$

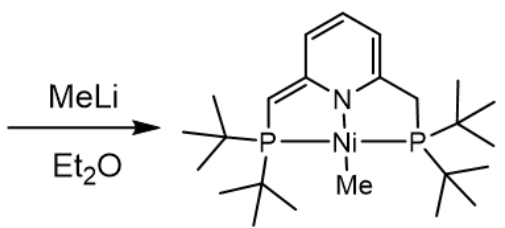

$\mathbf{L}$

Figure 2.24. An experimental approach for the dearomatization of pyridine based $\mathrm{Ni}^{2+}$ complexes (cf Figure 2.7). ${ }^{104}$

Reaction of MeLi with mononuclear complex $\mathbf{K}$ has been shown to be a selective reaction, which eventually led to the dearomatized complex L (Figure 2.24). In close experimental analogy, S. Samanta used MeLi with complex 2 to synthesize the complex 6 represented in (Figure 2.25). The complex could be crystalized from the crude reaction products and it was characterized by Xray diffraction. The synthetic accessibility of this complex was limited, and it could not be fully characterized. In the previous chapter, it was shown that there are currently few suitable Two-InOne pincer complexes for deprotonation and dearomatization studies (Figure 2.8 and Figure 2.21). The purpose of the following discussion was to demonstrate that complex 6 possibly had a corresponding dearomatized form within complex $\mathbf{5}$ ' (Figure 2.25). The following discussion was only supported by ${ }^{1} \mathrm{H}$ and ${ }^{31} \mathrm{P}$ NMR spectroscopy.
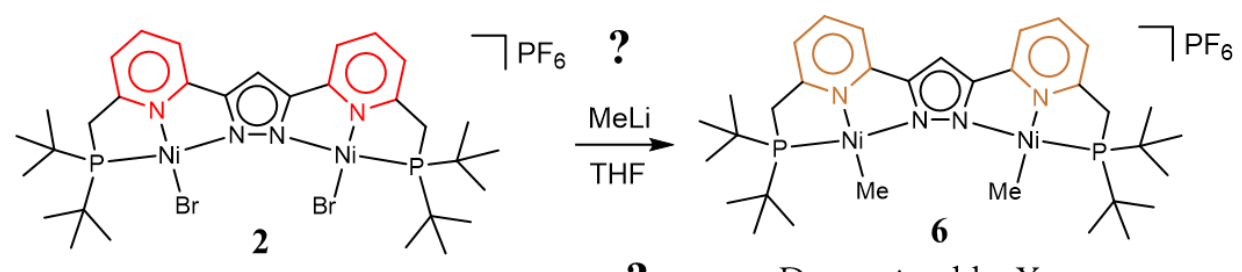

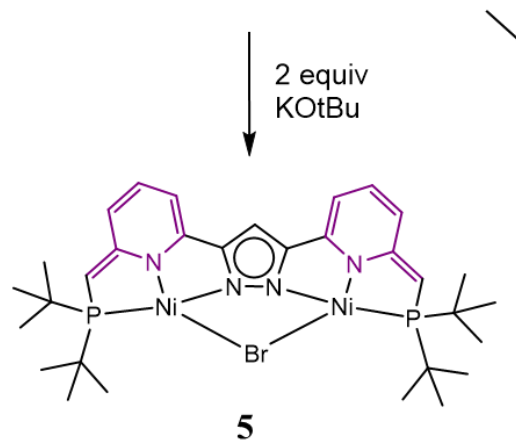

NMR

investigation

(This work)
Determined by X-ray

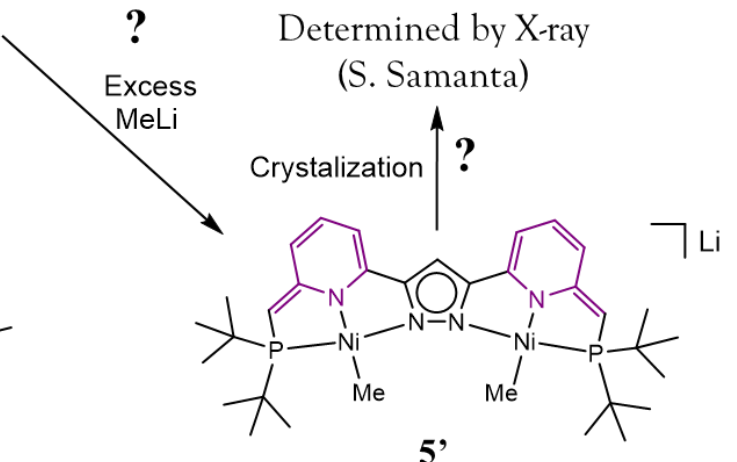

NMR investigation

(This work)

Figure 2.25. Representation of complexe $\mathbf{6}$ synthesized by S. Samanta. The possibility of a dearomatized complex $\mathbf{5}^{\prime}$ 'is discussed in this work. 
The ${ }^{31} \mathrm{P}$ NMR spectrum of complex 6 was shown in Figure 2.27. A ${ }^{31} \mathrm{P}$ NMR signal at $-144 \mathrm{ppm}$ for the $\mathrm{PF}_{6}{ }^{-}$anion and a signal at $73.5 \mathrm{ppm}$ were observed. The ${ }^{31} \mathrm{P}$ NMR signal of complex $\mathbf{6}$ was shifted $2.8 \mathrm{ppm}$ upfield in comparison to complex $\mathbf{2}$. This was probably due to the stronger donation of the $\mathrm{Me}^{-}$compared to the $\mathrm{Br}^{-}$ligands. The ${ }^{1} \mathrm{H}$ NMR of complex $\mathbf{6}$ was represented in Figure 2.26. The quality of the spectrum was low as only few crystalline materials of complex 6 was obtained following the procedure proposed by S. Samanta.

The reactivity of complex $\mathbf{2}$ with an excess of MeLi was investigated in this work. An excess of MeLi was added to a suspension of complex $\mathbf{2}$ in THF. The colour of the solution turned violet in the following minutes. It was interesting that the colour of the solution was very similar to the colour of complex $\mathbf{5}$ disssolved in THF. The complex was filtered off, and the solvent was removed under vacuum. The solid was washed with pentane and filtered again. The solid was then dissolved in THF- $\mathrm{d}^{8}$ for NMR analysis. The ${ }^{31} \mathrm{P}$ NMR spectrum was shown in Figure 2.27 (the top spectrum). The absence of a signal at $-144 \mathrm{ppm}$ indicated that the $\mathrm{PF}_{6}{ }^{-}$anion initially present in complex 2 was removed during the work up. A single signal at $57.3 \mathrm{ppm}$ was observed and likely indicated the presence of a symmetric complex. The ${ }^{31} \mathrm{P}$ NMR signal of the complex was shifted 16.2 ppm upfield in comparison to the ${ }^{31} \mathrm{P}$ NMR signal of complex $\mathbf{6}$, which indicated that the complex was probably richer in electrons. Such a shift possibly implicated a negatively charged complex. The ${ }^{1} \mathrm{H}$ NMR spectrum was represented in Figure 2.26. The quality of the spectrum was low, and the analysis of the ${ }^{1} \mathrm{H}$ NMR signals was limited to the analysis of chemical shifts. Interestingly, all ${ }^{1} \mathrm{H}$ NMR signals of the complex were shifted upfield in comparison to complex $\mathbf{6}$, which is reminiscent from the deprotonation of complex $\mathbf{2}$ to give complex $\mathbf{5}$. The typical pattern for two dearomatized pyridine moieties (like complex $\mathbf{5}$ in Figure 2.22) could be observed.

The difficulty to synthesize complexes $\mathbf{6}$ came from the fact, that it was not possible to evidence if the deprotonation of the ligand backbone by MeLi in complex 2 was occurring prior to the transfer of $\mathrm{Me}^{-}$to the $\mathrm{Ni}^{2+}$ ions as it was suggested in the literature (Figure 2.24). A selective route for the synthesis of complex $\mathbf{6}$ is still required. An attempt has been made to selectively obtain complex $\mathbf{6}$ from complex 2 by using a Grignard reagent (MeMgBr), but further investigation must be conducted to clarify this feature. The isolation of complex $\mathbf{5}$ ' appeared to be simpler, as an excess of MeLi directly led to the complex. However, attempts to crystalize the complex did not succeed. 


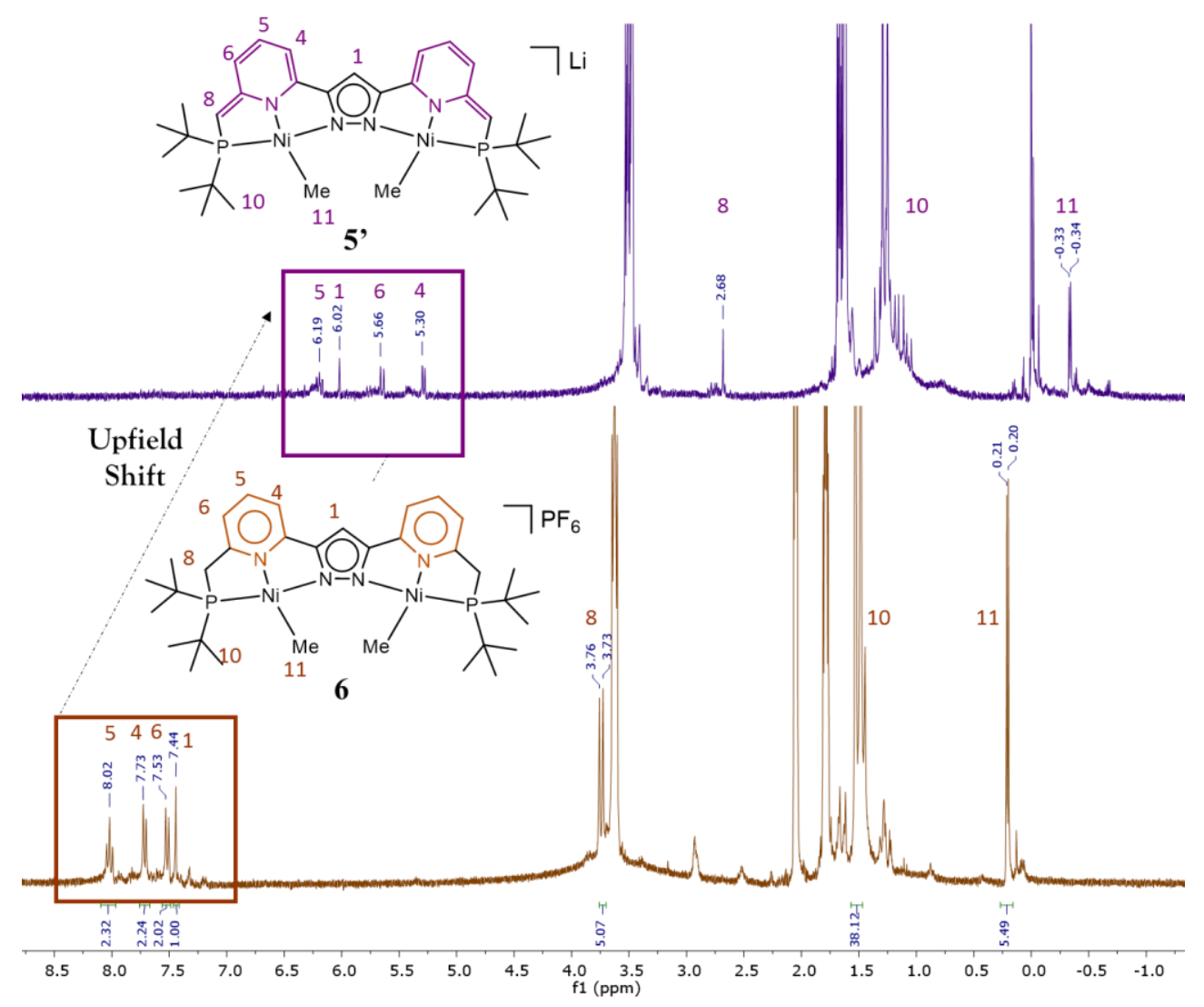

Figure 2.26. ${ }^{1} \mathrm{H}$ NMR of complexe $\mathbf{6}$ (bottom) and its hypothetical dearomatized form 5' (top spectrum) in THF-d ${ }^{8}$.

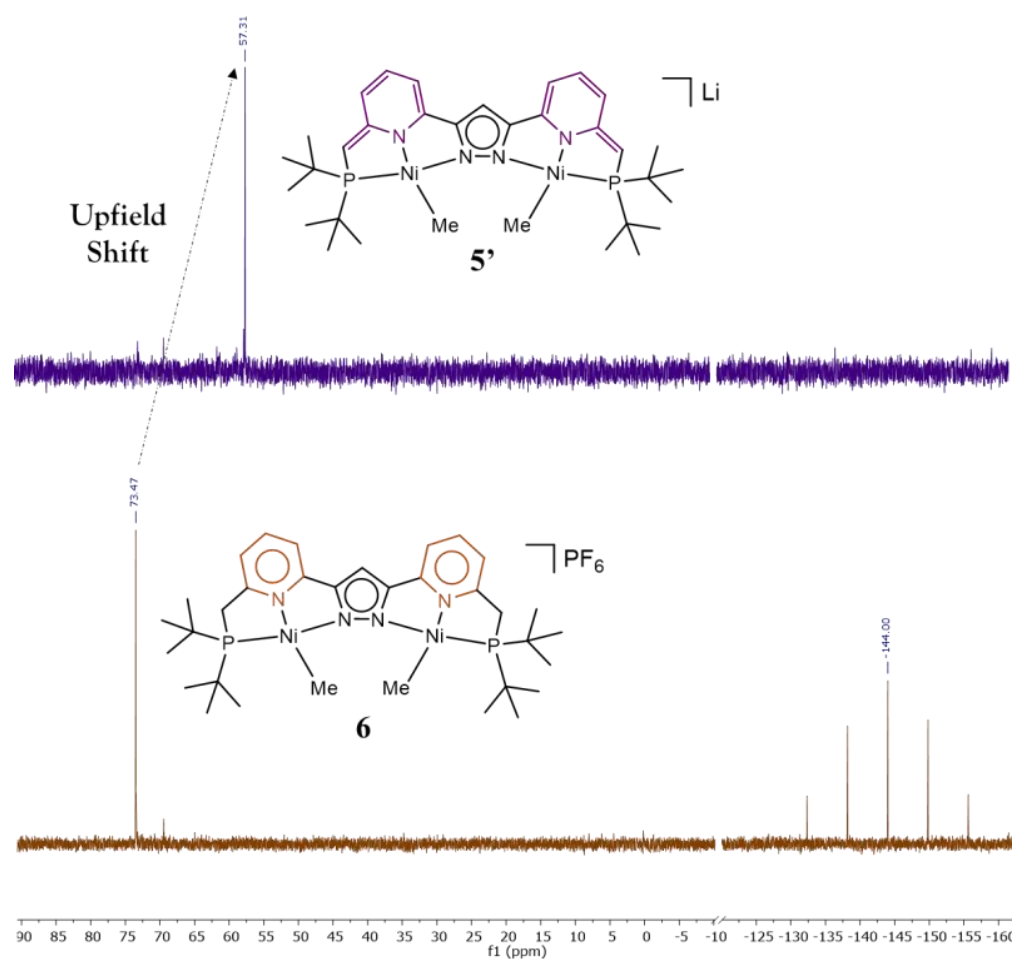

Figure 2.27. ${ }^{31} \mathrm{P}$ NMR spectra of complexe $\mathbf{6}$ (bottom) and its hypothetical dearomatized form 5' (top) in THF-d ${ }^{8}$. 


\subsection{Detection of a hydride signal}

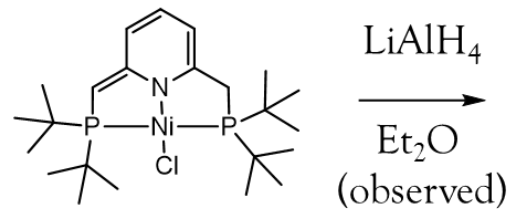

$\mathbf{N}$

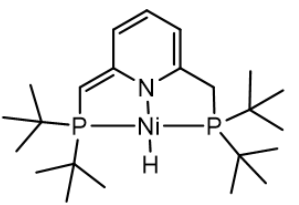

$\mathbf{P}$

Figure 2.29. Experimental approach to observe a Ni-H hydride resonance. ${ }^{104}$

The addition of $\mathrm{LiAlH}_{4}$ to the dearomatized complex $\mathbf{N}$ led to the observation of a ${ }^{1} \mathrm{H}$ NMR signal coming at $-18.4 \mathrm{ppm} .{ }^{104}$ The existence of complex $\mathbf{P}$ was first postulated. It was then confirmed by X-ray but much later. ${ }^{105}$ The method represented in Figure 2.29 exploited the absence of acidity of the $\mathrm{CH}_{2}$ side arm. Indeed, the side arm in the complex $\mathbf{N}$ has been deprotonated prior to addition of $\mathrm{LiAlH}_{4}$ which is strong base and could react with acidic protons. In that reaction, the hydride of $\mathrm{LiAlH}_{4}$ was transferred to the metal center to give the complex $\mathbf{P}$.

In the case of complex $\mathbf{2}$, the addition of $\mathrm{LiHBEt}_{3}$ led to a colour change from red to brown. A gas release was observed during the reaction. A signal at $4.55 \mathrm{ppm}$ was observed by ${ }^{1} \mathrm{H}$ NMR spectroscopy, which suggested that $\mathrm{H}_{2}$ formed during the reaction. However, the NMR signals corresponding to the spectra of those reaction were broad and possibly indicated the presence of paramagnetic compounds. It was not possible to clarify if the $\mathrm{H}_{2}$ was coming from the deprotonation of the side arm or if it came from a reductive elimination at a metal center.

In analogy to the reaction depicted in Figure 2.29, two equivalents of $\mathrm{LiHBEt}_{3}$ were added to complex $\mathbf{5}$ whereby the dark-violet colour of the sample turned into dark-brown. Interestingly, no gas release and no $\mathrm{H}_{2}$ resonance were observed this time. The NMR signals were broad, and it was not possible to well identify any species. However, heating the sample up to $60^{\circ} \mathrm{C}$ for several hours led to major changes in ${ }^{1} \mathrm{H}$ NMR signals. The heating of the sample gave rise to a new highfield ${ }^{1} \mathrm{H}\left\{{ }^{31} \mathrm{P}\right\}$ NMR signal located at $-19.53 \mathrm{ppm}$ (Figure 2.30), which likely indicated the formation of metal-hydrogen bond. 

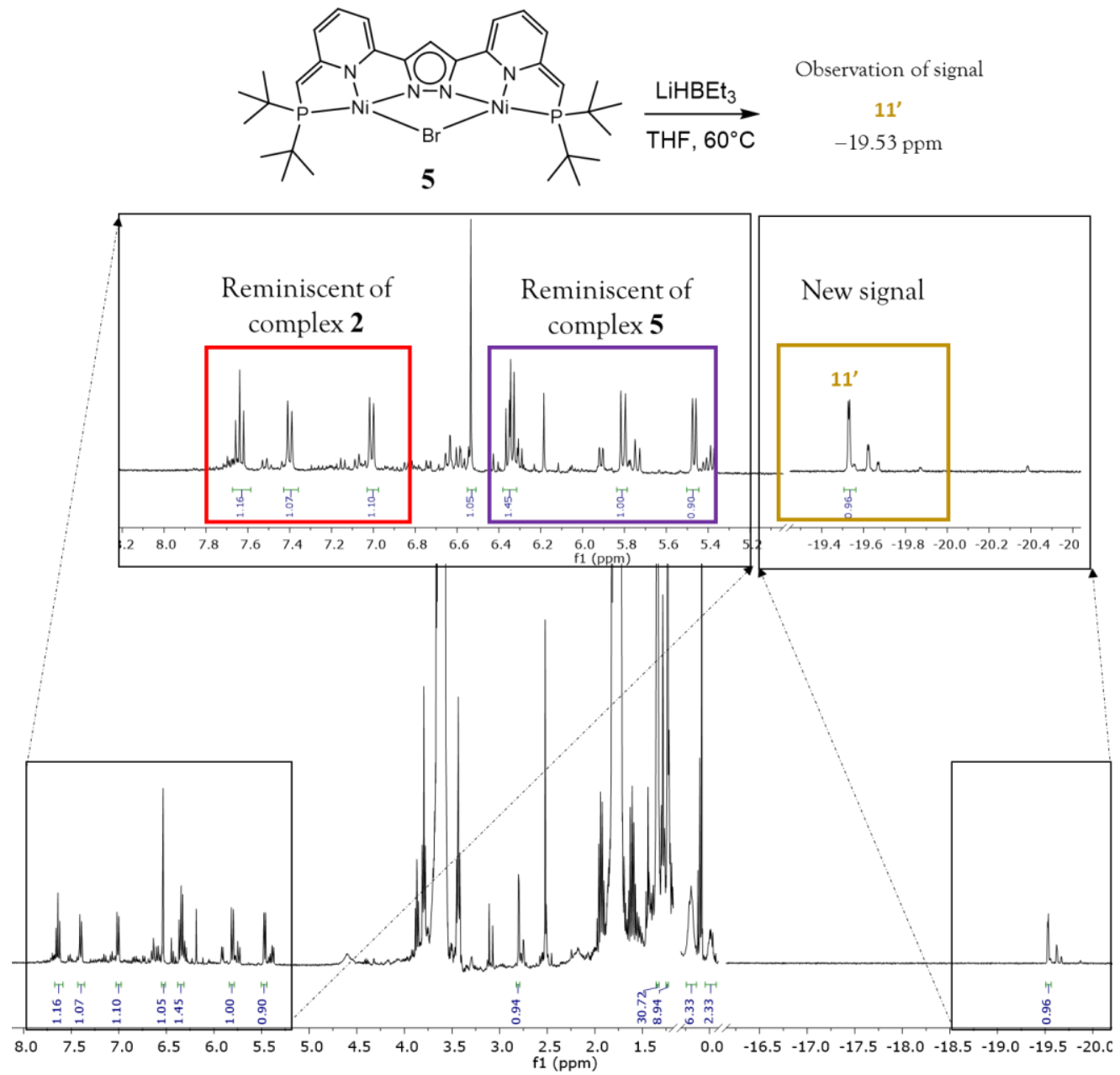

Figure 2.30. ${ }^{1} \mathrm{H}\left\{{ }^{31} \mathrm{P}\right\}$ NMR spectrum resulting from the reaction of complex $\mathbf{5}$ with $\mathrm{LiHBEt}_{3}$ in THF- $\mathrm{d}^{8}$.

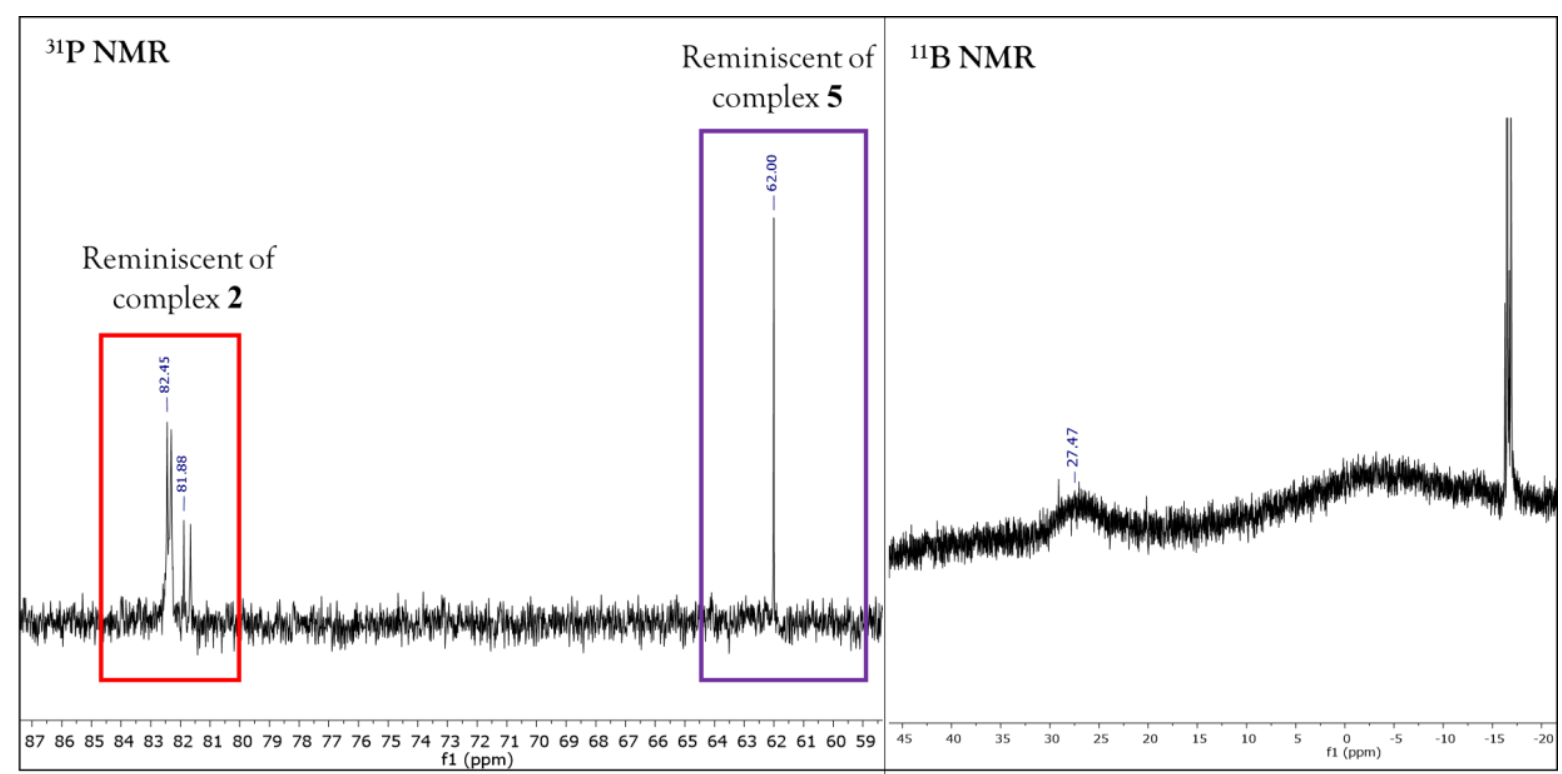

Figure 2.31. ${ }^{31} \mathrm{P}$ NMR and ${ }^{11} \mathrm{~B}$ NMR spectra resulting from the reaction of complex $\mathbf{5}$ with $\mathrm{LiHBEt}_{3}$ in THF-d ${ }^{8}$. 
It was observed that ${ }^{1} \mathrm{H}\left[{ }^{31} \mathrm{P}\right\}$ NMR signals in the aromatic region had an integral almost equal to 1 with respect to the highfield signal at $-19.53 \mathrm{ppm}$ (Figure 2.30). The chemical shift of the signals in the aromatic region was similar to a combination of NMR signals of complex $\mathbf{2}$ (red box) and complex 5 (violet box) within the same spectrum. Only one new $\mathrm{C}-\mathrm{H}$ signal for a pyrazole was detected. It suggested the possibility of an asymmetric compound with an aromatic pyridine on one side of the complex and a dearomatized pyridine one on the other side. This hypothesis was supported by ${ }^{31} \mathrm{P}$ NMR spectroscopy (Figure 2.31) which displayed signals in the chemical range of the aromatic (red box) and dearomatized (violet box) pyridine of complex 2 and $\mathbf{5}$ respectively. It was interesting to notice that ${ }^{11} \mathrm{~B}$ NMR displayed a broad signal at 27.47 ppm (Figure 2.31 (right)). The shift of this signal falls in the same range as a previously reported Ru complexe, on which a B-C bond formed on the side arm after a polar B-H bond activation $\left({ }^{11}\right.$ B NMR: broad signal at $\left.30.67 \mathrm{ppm}\right) .{ }^{93}$

To summarize, the addition of $\mathrm{LiHBEt}_{3}$ to complex $\mathbf{5}$ led to the appearance of an upfield ${ }^{1} \mathrm{H}$ NMR signal at $-19.53 \mathrm{ppm}$ after several hours at $60^{\circ} \mathrm{C}$. A broad ${ }^{11} \mathrm{~B}$ NMR signal at $27.47 \mathrm{ppm}$ was observed and it was possible to observe the rearomatization of a pyridine moiety. Unfortunately, the crystallization of this complex did not succeed, and it was not further characterized. In the next chapter, a doubly dearomatized dihydride dinickel complex was successfully isolated and characterized by circumventing the use of $\mathrm{LiHBEt}_{3}$.

\subsection{Conclusion}

In conclusion, this chapter demonstrated the feasible double deprotonation of complex $\mathbf{2}$ concomitant with the double dearomatization of the two pyridines involved in the complex. It was evidenced by typical upfield shifts in the ${ }^{1} \mathrm{H}$ NMR signals of aromatic protons. Generally, it was shown that the deprotonation caused most of the NMR signals of the ${ }^{1} \mathrm{H},{ }^{31} \mathrm{P},{ }^{13} \mathrm{C}$ nuclei to be shifted upfield. The detection of a ${ }^{1} \mathrm{H}$ high field resonance at $-19.53 \mathrm{ppm}$ after the addition of $\mathrm{LiHBEt}_{3}$ to complex $\mathbf{5}$ possibly indicated the existence of a stable metal-hydrogen specie which was however not structurally elucidated. In the next chapter, the methodology for the synthesis of $\mathrm{Ni}-\mathrm{H}$ complex was improved in order to isolate and stabilize such a compound. 


\section{Chapter 3: A doubly dearomatized dinickel dihydride complex and the elusive cooperation of the ligand.}

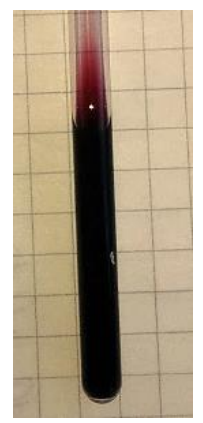

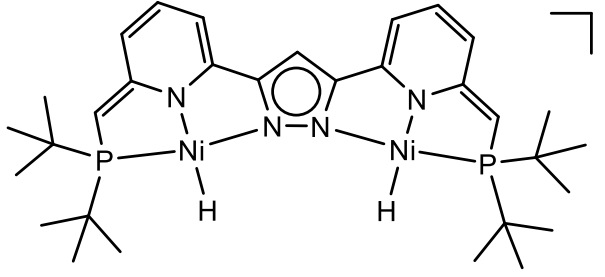

Diamagnetic Bordeaux

$$
\mathrm{S}=0
$$

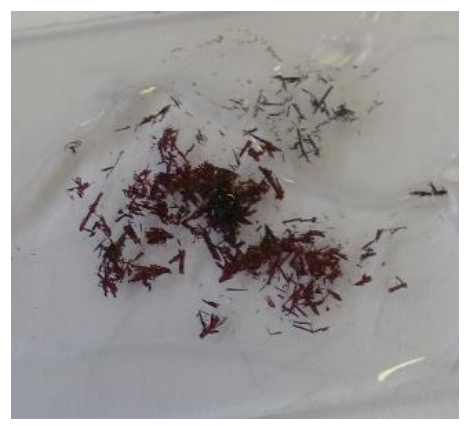

\subsection{Introduction: Biorelevance of the nickel hydride motif for the}

anthropogenic and non-anthropogenic production of $\mathrm{H}_{2}$.

A major challenge of mankind in the 21th century is the resolution of environmental problems caused by its inexhaustible growing needs in energy. In the context of chemical process engineering for renewable energy conversion, dihydrogen, the simplest diatomic molecule composed of the most abundant element in the universe, represents a viable alternative to fossil fuels. ${ }^{119}$ Since the beginning of the 20th century, dihydrogen is mainly generated as synthetic gas (Syngas) from non-renewable natural gas by steam reforming. ${ }^{120}$ Nickel is the preferred catalyst employed for this process. ${ }^{121}$ In 2007 , the world production of dihydrogen was reported to be between 45 and $50 \mathrm{Mt}$, of which only $4 \%$ was generated by electrolysis. ${ }^{120}$ Applications of $\mathrm{H}_{2}$ gas can majorly be found for the hydrogenation of organic substrates in the plastic, pharmaceutic, alimentary industries, etc...
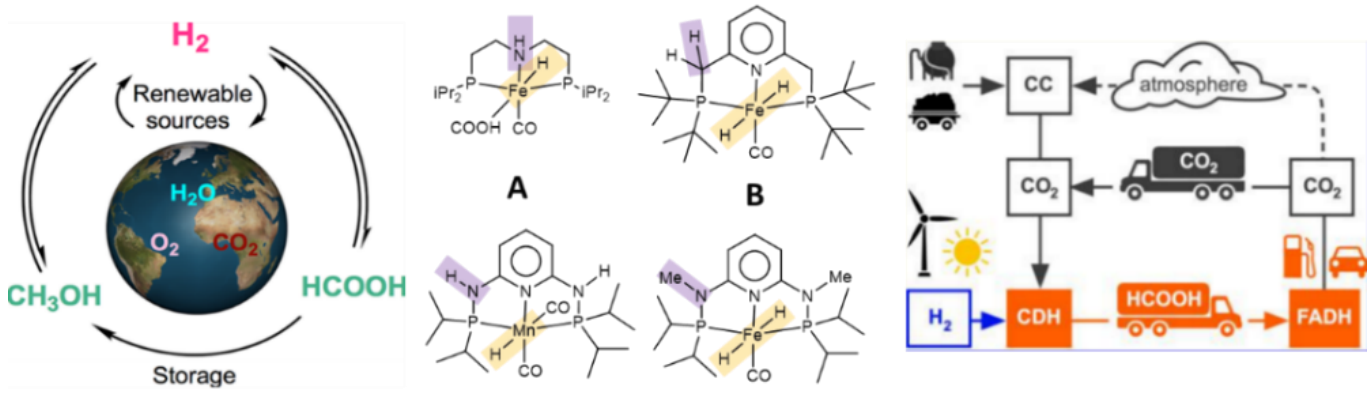

C

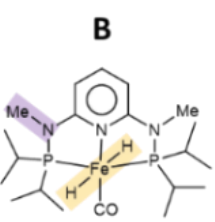

$\mathrm{X}-\mathrm{Y}$ side arm $+\mathrm{M}-\mathrm{H}$ moiety

Figure 3.1. Catalytic transformations for the storage of $\mathrm{H}_{2}$. Some complexes which promote this reaction are represented in $\mathbf{A},{ }^{127} \mathbf{B},{ }^{128} \mathbf{C}^{129}$ and $\mathbf{D} .{ }^{130}$ The left ${ }^{126}$ and right ${ }^{132}$ pictures have been taken from the literature. 
However, $\mathrm{H}_{2}$ has recently received more attention as a promising fuel for the transportation sector. A society with a hydrogen economy is not viable with unsustainable means of $\mathrm{H}_{2}$ production. The replacement of transportation combustion systems and the electrification of the overall transport sector for individual civil transportation render the promise of fuel-cell based $\mathrm{H}_{2}$ vehicles possible. ${ }^{120}$

A successful demonstration of fuel cell bus programs in 2004 was conducted in California by SunLine Transit Agency and in Europe by CUTE (Clean Urban Transportation for Europe). ${ }^{120}$ Absence of NOx and nanoparticle release was diagnosed. The status of fuel cell electric vehicles has been detailed in 2012. ${ }^{122}$ A breakthrough has been observed in 2018 with the development of the first hydrogen powered train: "Coradia iLint". It has been developed by ALSTOM. It has been approved for civil transportation in Germany and is henceforth operational in Lower Saxony. ${ }^{123}$ Because of the growing awareness of the role of $\mathrm{H}_{2}$ in the current transition of energy, the US Senate officially voted for the creation of the "Hydrogen National $\mathcal{E}$ Fuel Cell Day" in the United State of America in 2018. ${ }^{124}$ Technological progress is now actively directed to develop efficient $\mathrm{H}_{2}$ storage methods. In this regard, formic acid represents an interesting candidate. ${ }^{125}$ The concept is currently being pioneered by Team FAST located in Eindhoven with the development of an innovative $\mathrm{H}_{2}$ fuel cell bus program called HYDROZINE, which is based on the decomposition of $\mathrm{HCOOH}$ to $\mathrm{CO}_{2}$ and $\mathrm{H}_{2}$.

The possibilities to catalytically achieve such reactions has been reviewed recently. ${ }^{126}$ The implication of a $\mathrm{X}-\mathrm{H}(\mathrm{X}=\mathrm{C}$ or $\mathrm{N})$ polar bond in the side arm of pincer complexes has been studied. Complex $\mathbf{A}^{127}$ was studied for the dehydrogenation of formic acid. Complexes $\mathbf{B}^{128}$ and $\mathbf{C}^{129}$ were studied for the hydrogenation of carbon dioxide (Figure 3.1). Complex B was chemically dearomatized, but this study did not unequivocally implicate the participation of the dearomatized form of complex $\mathbf{B}$ in the catalytic cycle. In case of complex $\mathbf{C}$, the participation of the $\mathrm{N}-\mathrm{H}$ moiety played an important role for catalytic efficiency, however the dearomatization of the complex possibly induced by the deprotonation of the side arm was only explored by calculations. While the side arm of complexes A, B and C likely led to beneficial metal-ligand cooperativity in the hydrogenation of carbon dioxide or its reverse reaction, it was demonstrated to not always be a necessary feature in the case of complex $\mathbf{D} .{ }^{130}$ Thus, the influence of the side arm in pincer complexes in catalytic cycles led to diverse impacts. Hydrides of transition metals are relevant intermediates involved in the catalytic hydrogenation of $\mathrm{CO}_{2} \cdot{ }^{131}$ In 2017 , a study mentioned molecular catalyst cost issues that would be involved for the functionalization of citizen vehicles base on such reactions. ${ }^{132}$ Cheap metal-based complexes that can efficiently activate $\mathrm{H}_{2}$ with efficiency for industrial applications are now required. 
The culture of microorganism containing hydrogenase enzymes is a possible strategy to circumvent the anthropogenic production of $\mathrm{H}_{2} \cdot{ }^{133}$ In 1931 , hydrogenases present in a variety of bacteria were found to activate molecular hydrogen. ${ }^{134}$ Different environmental conditions such as the atmospheric concentration in $\mathrm{O}_{2}$ or the soil constitution $(\mathrm{pH}$, presence of metals, temperature) contributed to the emergence of different niches of bacteria having the same gene or DNA sequence encoding for the hydrogenase enzyme. ${ }^{135}$ A phylogenetic study revealed however that those bacteria involve a variety of metabolisms based on the oxidation of methane, ammonia or nitrite. ${ }^{136}$ At the present time there are three known types of hydrogenase enzymes : $[\mathrm{Fe}],[\mathrm{FeFe}]$ and $[\mathrm{NiFe}]$ hydrogenases. ${ }^{137}$ The presence of nickel and iron ions in the $[\mathrm{NiFe}]$ hydrogenase was confirmed based on the X-ray structure of desulfovibrio gigas in $1995 .{ }^{138}$ The steric crowd generated by the hundreds of amino acids of the protein was hiding the active site of the enzyme, rendering the characterization of the metallic core and its reactivity challenging. The current state of the art for mechanistic understanding of $\mathrm{H}_{2}$ activation by those hydrogenases was reviewed in literature. ${ }^{139,140,141}$

Functional and structural models have been synthetized by biochemists and chemists in order to better understand how $\mathrm{H}_{2}$ can reversibly be split at the reactive site. Functional models were designed by Dubois with $\mathrm{Ni}^{2+}$ complexes supported by phosphine ligands and lead to conclusive results about the importance of surrounding hydrogen bonds around the metallic site for an efficient catalytic oxidation of $\mathrm{H}_{2}$ (Figure 3.3, complex $\mathbf{E}$ ). ${ }^{142}$ The mechanism later confirmed the formation of nickel hydride intermediates $\{\mathrm{Ni}-\mathrm{H}\}$ in the catalytic cycle. ${ }^{143}$ Other phosphine based $\mathrm{Ni}^{2+}$ complexes were found to be efficient catalyst for the reduction of $\mathrm{H}_{2} \cdot{ }^{144,145}$ Structural mimicry of [ $\mathrm{NiFe}]$ enzymes is well documented by synthetic heterobimetallic complexes involving a $\{\mathrm{Ni}-\mathrm{H}$ Fe) motif ${ }^{146}$ (Figure 3.3, complexes $\mathbf{F},{ }^{147} \mathbf{G},{ }^{148} \mathbf{H},{ }^{149} \mathbf{I}^{150}$ ). The total number of complexes featuring a $\{\mathrm{Ni}-\mathrm{H}\}$ motif in pincer and non-pincer systems was reported to be around 165 in $2016 .{ }^{151}$ Recently, it was shown that Ni pincer complexes are not only artificial motifs created in lab fumehoods but are also used by nature as it can be observed in the molecular structure of the cofactor of lactate racemase. ${ }^{152} \mathrm{~A}$ model of this cofactor has already been synthesized in order to investigate the involvement of a $\{\mathrm{Ni}-\mathrm{H}\}$ intermediate in the isomerization process (Figure 3.2). ${ }^{153}$

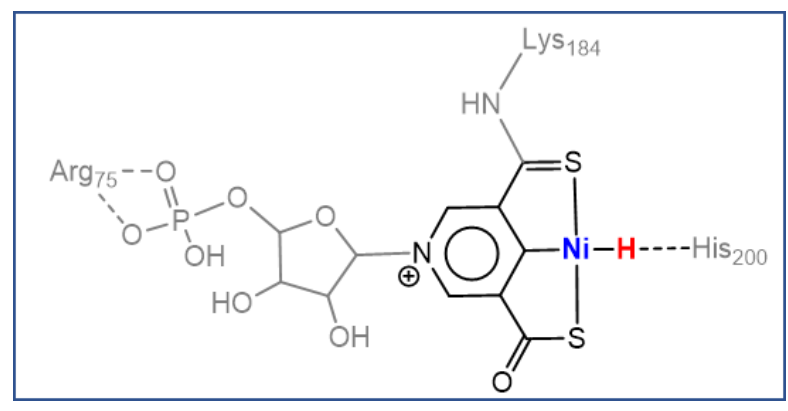

Figure 3.2. Representation of the Ni cofactor in lactate racemase. ${ }^{153}$ 


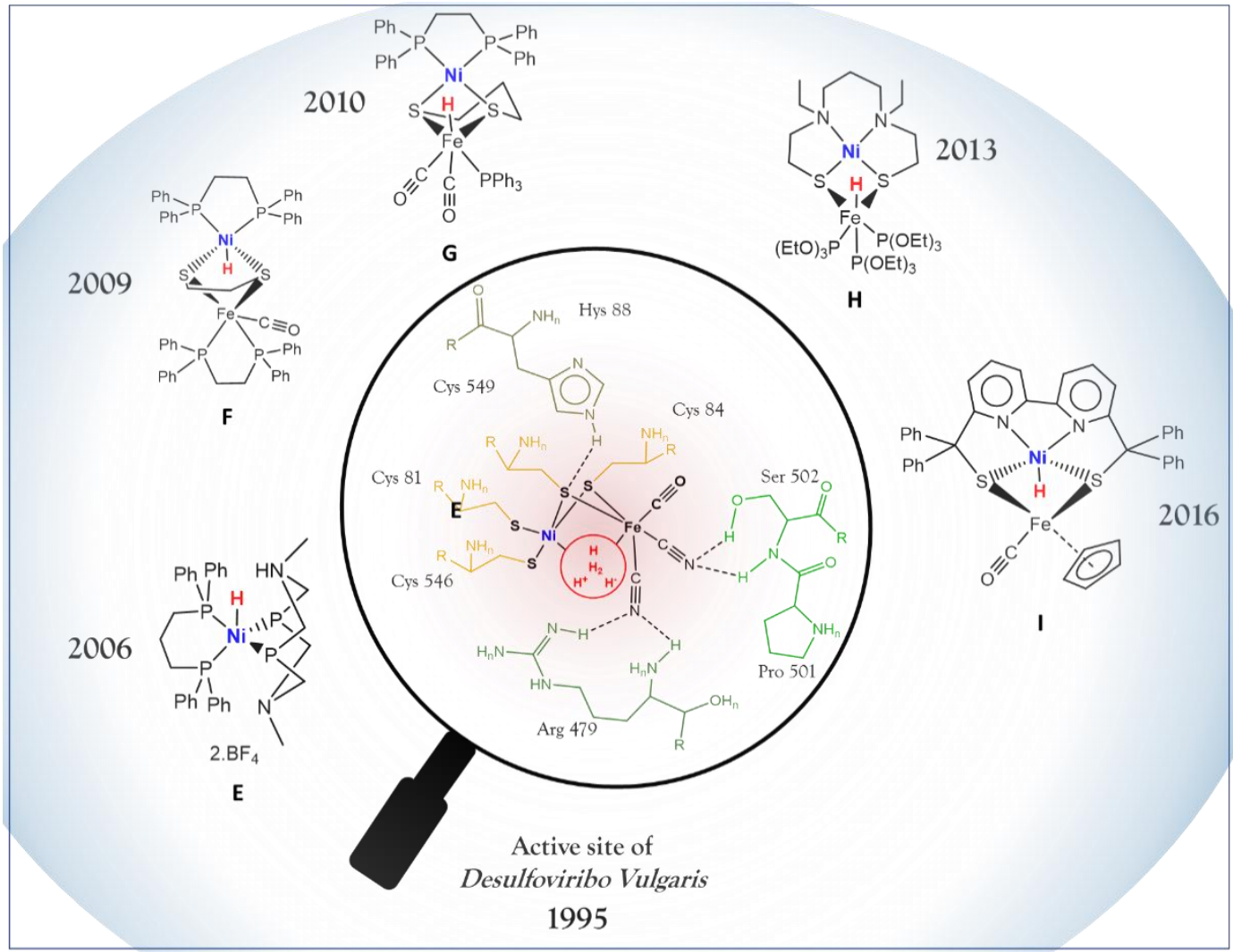

Figure 3.3. Representation of the active site in [NiFe] hydrogenase according to published X-ray data. Structural and functional models involving $3 \mathrm{~d}$ metals with at least one $\mathrm{Ni}$ atom are represented around it.

In close analogy to the system that is going to be developed in the following chapter, the dihydride system developed by D. Manz (Figure 3.3) offered the possibility to make an interesting comparative study in the activation of $\mathrm{H}_{2} \cdot{ }^{154}$ This complex has been reported to reductively bind small substrates. ${ }^{155,156}$ The two $\{\mathrm{Ni}-\mathrm{H}\}$ moiety contained in the dinuclear complex formed $\mathrm{H}_{2}$ likely by a reductive elimination reaction, which led to the description of this dihydride complex as a masked dinickel(I) species. Then $\mathrm{H}_{2}, \mathrm{O}_{2}$ or $\mathrm{NO}$ could be reduced by the two $\mathrm{Ni}^{\mathrm{I}}$ ions, which in turn reoxidized into $\mathrm{Ni}^{\mathrm{II}}$.

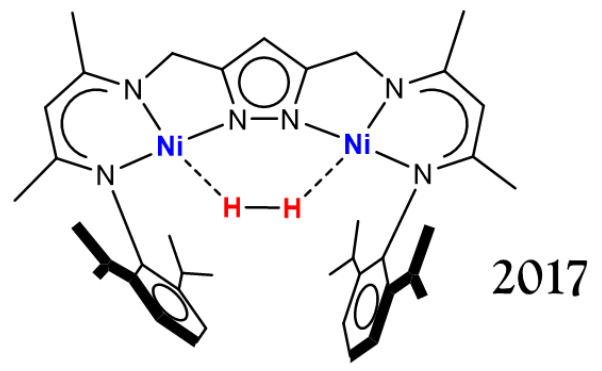

Figure 3.3. Representation of the dihydride system developed by D. Manz. ${ }^{154}$ 


\subsection{Isolation of a doubly dearomatized dihydride dinickel complex}

As studied in the previous chapter, addition of two equivalents of $\mathrm{KO}^{t} \mathrm{Bu}$ led to double dearomatization of complex $\mathbf{2}$. The alternative use of $\mathrm{KH}$ as deprotonating agent was preferred, as it led selectively to the same result, but with $\mathrm{H}_{2}$ as the only side product of the reaction. Increasing the equivalents of $\mathrm{KH}$ gave rise to a high field ${ }^{1} \mathrm{H}$ NMR resonance peak located at -20.20 ppm, consistent with the formation of a new product derived from complex $\mathbf{5}$. The addition of a stoichiometric amount of $[2,2,2]$ cryptand in the solution led to the formation of a positively charged potassium/cryptand adduct $\mathrm{K}[2,2,2]+$, which then crystalized to give the new complex $\mathrm{K}[2,2,2]\left[\mathrm{LNi}_{2}(\mathrm{H})_{2}\right](\mathbf{7})$. Crystals of complex $\mathbf{7}$ were analysed by X-ray diffraction, NMR and IR spectroscopy, ESI-MS and EA. The dearomatized character of this unique complex, which also involved two hydride ligands was the first among other dearomatized or hydride Two-In-One pincer complexes to be structurally elucidated and fully characterized. The method employed for the synthesis of this complex could be adapted to the Rh complex synthesized by Gers-Barlag (adaptation of the reaction represented in Figure 2.21), which eventually crystallized and gave the metrical data discussed in part 2.1. ${ }^{157}$

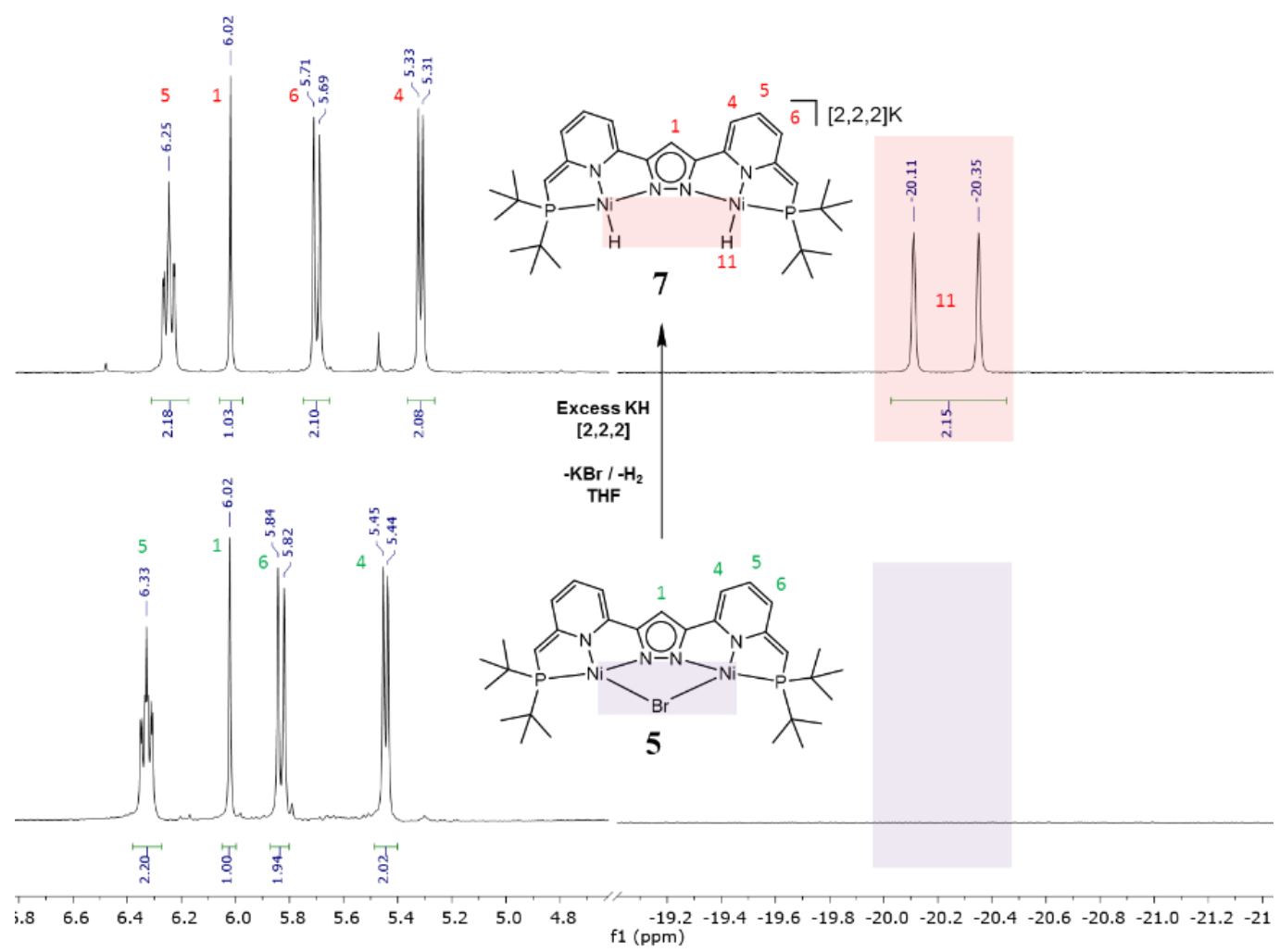

Figure 3.4. ${ }^{1} \mathrm{H}$ NMR spectra in THF-d ${ }^{8}$ of complex 5 (bottom) and 7 (top), indicating the formation of a new upfield resonance. Spectra are truncated for clarity. 

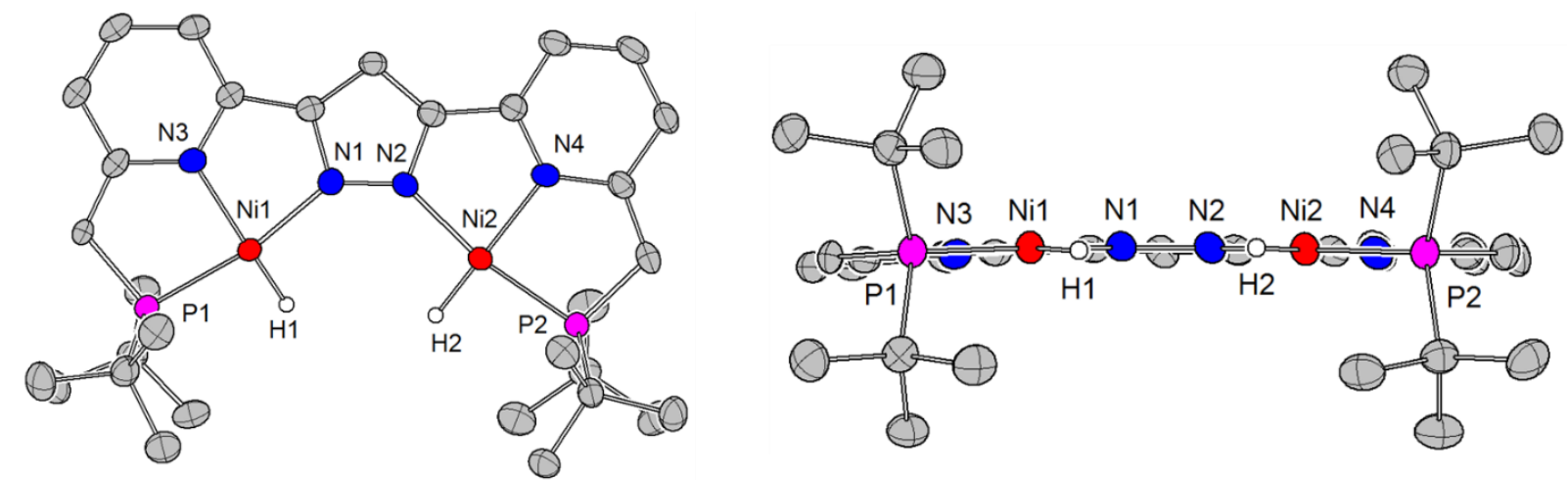

Figure 3.5. Molecular structure (thermal displacement ellipsoids shown at $50 \%$ probability) of the anion of complex 7. Most of the hydrogen atoms and all cations have been omitted for clarity. Left: top view of the molecular structure. Right: front view of the molecular structure

\begin{tabular}{cc|cc}
\hline \multicolumn{2}{l|}{ Bond Lengths around $\mathrm{Ni}(1) / \AA$} & Bond Lengths around $\mathrm{Ni}(2) / \AA$ \\
\hline & & & \\
\hline $\mathrm{Ni}(1)-\mathrm{N}(3)$ & $1.903(4)$ & $\mathrm{Ni}(2)-\mathrm{N}(2)$ & $1.901(4)$ \\
\hline $\mathrm{Ni}(1)-\mathrm{N}(1)$ & $1.911(4)$ & $\mathrm{Ni}(2)-\mathrm{N}(4)$ & $1.908(4)$ \\
\hline $\mathrm{Ni}(1)-\mathrm{P}(1)$ & $2.1220(13)$ & $\mathrm{Ni}(2)-\mathrm{P}(2)$ & $2.1135(13)$ \\
\hline $\mathrm{Ni}(1)-\mathrm{H}(1)$ & $1.22(6)$ & $\mathrm{Ni}(2)-\mathrm{H}(2)$ & $1.31(10)$ \\
\hline
\end{tabular}

Table 3.1. Selected bond lengths

The two pyridine rings of complex $\mathbf{7}$ were dearomatized. Similar to the example given in Figure 2.3, metrical data analysis revealed localized $\mathrm{C}=\mathrm{C}$ and $\mathrm{C}-\mathrm{C}$ bonds in comparison to complex $\mathbf{2}$, where the bond lengths of the pyridine were averaged between $\mathrm{C}-\mathrm{C}$ and $\mathrm{C}=\mathrm{C}$ bond (Figure 3.5). Even if the pyridines of the complex were dearomatized, the overall planarity adopted by the ligand backbone in the crystal structure (Figure 3.5) suggested that the ligand backbone was a fully conjugated system. The absence of steric clash between the two small terminal hydride ligands allowed the flattening of the backbone (Figure 2.12) and to a smaller intermetallic $\mathrm{Ni} \cdot \cdots \mathrm{Ni}$ distance (4.156 ̊) compared to complex $\mathbf{2}$ and $\mathbf{4}$. While the bond lengths between the nitrogen of the pyridine and the nickel ion were nearly the same before and after dearomatization (average for complex $21.901 \AA$ vs $1.906 \AA$ for complex 7), the P-Ni bond length was noticeably shorter (Average for complex $22.175 \AA$ vs $2.118 \AA$ A complex 7).

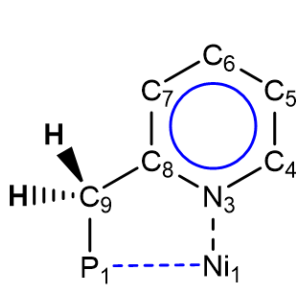

Aromatic character

In complex 2

$$
\begin{aligned}
& \mathrm{P}_{1}-\mathrm{Ni}_{1}=2.1792(9) \\
& \mathrm{P}_{1}-\mathrm{C}_{9}=1.833(4) \\
& \mathrm{C}_{9}-\mathrm{C}_{8}=1.500(5) \\
& \mathrm{C}_{8}-\mathrm{C}_{7}=1.391(5) \\
& \mathrm{C}_{7}-\mathrm{C}_{6}=1.379(5) \\
& \mathrm{C}_{6}-\mathrm{C}_{5}=1.385(5) \\
& \mathrm{C}_{5}-\mathrm{C}_{4}=1.387(4) \\
& \mathrm{C}_{4}-\mathrm{N}_{3}=1.362(4)
\end{aligned}
$$

$$
\begin{aligned}
& \mathrm{P}_{1}-\mathrm{Ni}_{1}=2.1220(13) \\
& \mathrm{P}_{1}-\mathrm{C}_{9}=1.781(4) \\
& \mathrm{C}_{9}-\mathrm{C}_{8}=1.373(7) \\
& \mathrm{C}_{8}-\mathrm{C}_{7}=1.445(6) \\
& \mathrm{C}_{7}-\mathrm{C}_{6}=1.360(8) \\
& \mathrm{C}_{6}-\mathrm{C}_{5}=1.409(7) \\
& \mathrm{C}_{5}-\mathrm{C}_{4}=1.381(7) \\
& \mathrm{C}_{4}-\mathrm{N}_{3}=1.358(6)
\end{aligned}
$$<smiles>C1=Cn2[nH]n3ccccc3ccc21</smiles>

Dearomatized character in complex 7

Figure 3.6. Metrical analysis of $\mathrm{C}=\mathrm{C}$ and $\mathrm{C}-\mathrm{C}$ bonds before (blue) and after (red) dearomatization of the pyridine. 
The ${ }^{1} \mathrm{H}$ NMR spectrum of complex 7 is shown in Figure 3.7. Like the other dearomatized Ni complex $\mathbf{5}$, the ${ }^{1} \mathrm{H}$ NMR signals of the pyridine were shifted upfield in the typical area of olefinic resonances $\{6.28-5.35\} \mathrm{ppm}$ (Figure 3.7), which indicated dearomatization. The proton 5 in para position of the pyridine displayed a long distance ${ }^{5} \mathrm{~J}_{\mathrm{P}-\mathrm{H}}=1.95 \mathrm{~Hz}$ with the phosphorus atom. The hydride signal (proton 11) appeared at $-20.20 \mathrm{ppm}$ as a doublet with a large coupling constant of ${ }^{2} \mathrm{~J}_{\mathrm{P} \cdot \mathrm{H}}=96 \mathrm{~Hz}$. The coupling constant was evidenced by a ${ }^{31} \mathrm{P}\left\{{ }^{1} \mathrm{H}\right\}$ decoupling measurement. Typical values for a ${ }^{2} \mathrm{~J}_{\mathrm{P}-\mathrm{H}}$ coupling hydride cis to a phosphorus atom coordinated to nickel(II) are in the range of 50 to $70 \mathrm{~Hz} .{ }^{151}$ The complex 7 featured a single ${ }^{31} \mathrm{P}$ NMR signal for the two equivalent phosphorus atoms located at $84.3 \mathrm{ppm}$. All the ${ }^{1} \mathrm{H}$ NMR signals in complex 7 have been shifted upfield in comparison to complex $\mathbf{5}$, suggesting a higher electron density delocalized in the backbone of the complex. Reasons for the electron enrichment might be due to the stronger $\sigma$ donating hydride ligand instead of bromide anion. The anionic charge of the complex could also be involved in the upfield shift of the NMR signals. Curiously, the ${ }^{31} \mathrm{P}$ NMR signals has been shifted $16.3 \mathrm{ppm}$ downfield. This deshielding effect could be attributed to the shorter $\mathrm{Ni}-\mathrm{P}$ bond. Indeed, the crystallographic data of the solid state indicated that the $\mathrm{Ni}-\mathrm{P}$ bond length was the shortest among complexes $\mathbf{1}, \mathbf{2}, \mathbf{3}, \mathbf{4}$. A correlation between the hydride proton 11 and the proton 8 on the side arm has been observed in two dimensional ${ }^{1} \mathrm{H} /{ }^{1} \mathrm{H}$ COSY NMR (Figure 3.8). It was further characterized by HSQC, ${ }^{1} \mathrm{H} /{ }^{13} \mathrm{C} \mathrm{HMBC}$, NOESY experiments. Detailed characterization can be found in the appendices.

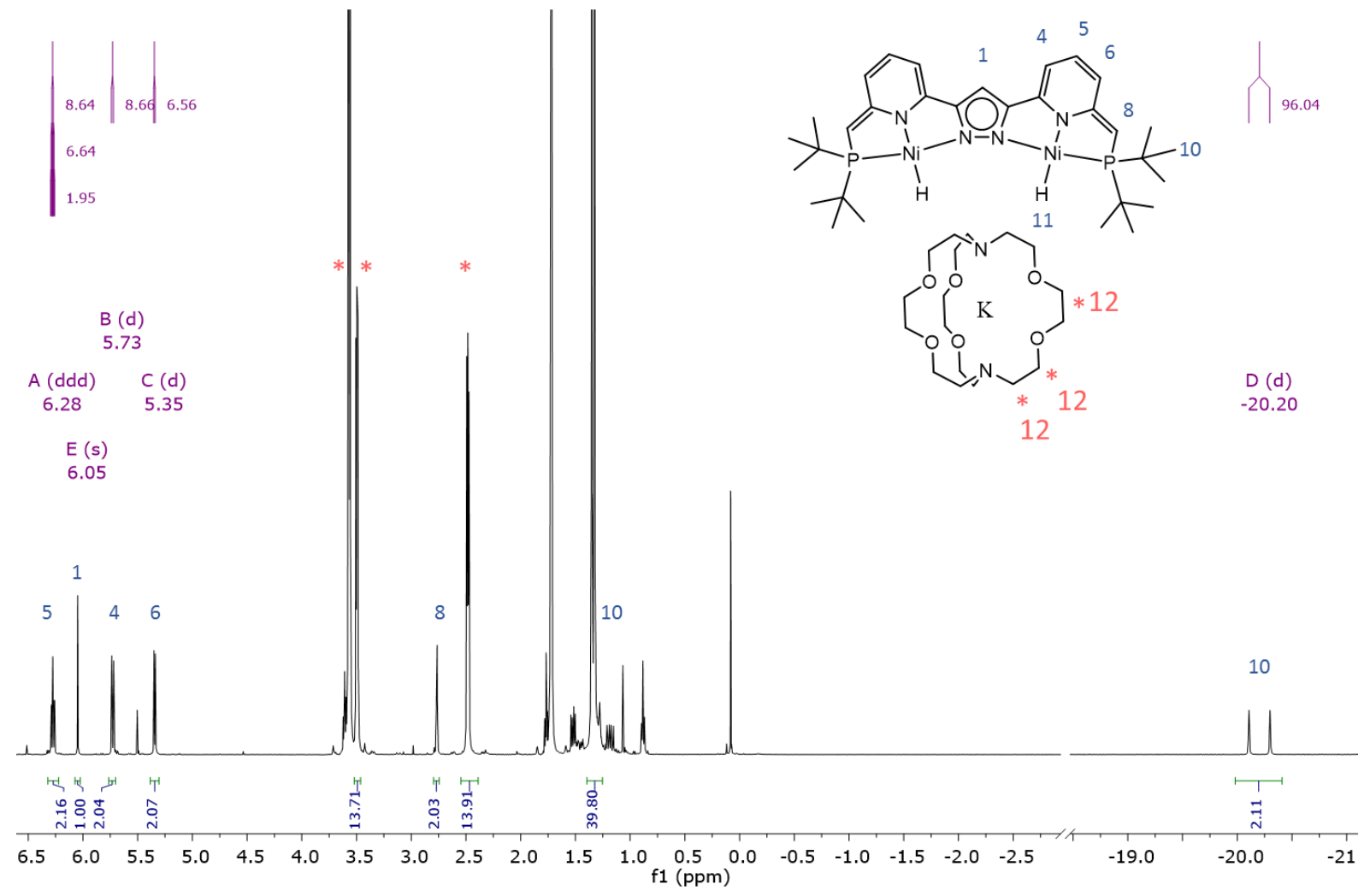

Figure 3.7. ${ }^{1} \mathrm{H}$ NMR spectrum of complex 7 in THF-d ${ }^{8}(500 \mathrm{MHz})$. 


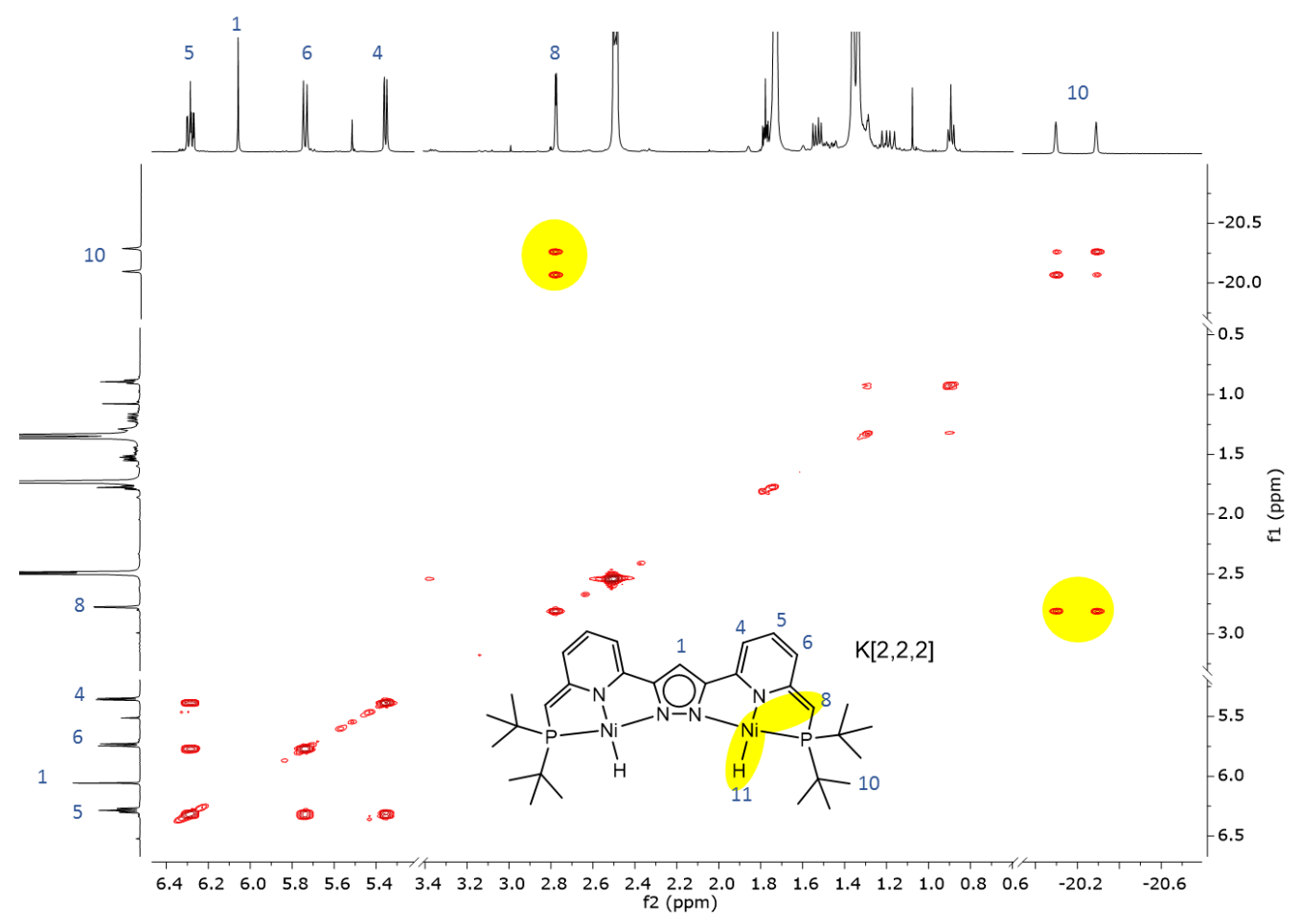

Figure 3.8. ${ }^{1} \mathrm{H} /{ }^{1} \mathrm{H}$ COSY NMR spectrum of complex 7 in THF-d ${ }^{8}(500 \mathrm{MHz})$, highlighting the correlation between the proton 8 and 11 .

The dearomatized state of complex $\mathbf{7}$ was confirmed by infrared spectroscopy. ATR-IR spectra of crystalline material of the free ligand VIII, complex 1, 2 and $\mathbf{7}$ were superimposed in Figure 3.10. Absorption bands located in the region $\left\{1575-1555 \mathrm{~cm}^{-1}\right.$ (yellow mark) are attributed to the coordination mode of the pyridine. As can be seen, the absorption band has shifted by about 23 $\mathrm{cm}^{-1}$ after dearomatization in complex 7 . The IR analysis of such phenomenon has already been made in $\mathrm{Ni}$ and $\mathrm{Cu}$ pincer complexes. ${ }^{104,158}$ Similar results are also obtained by dearomatization of the Rh complex synthesized by Gers-Barlag (Figure 3.9), but it was previously not discussed. ${ }^{52}$

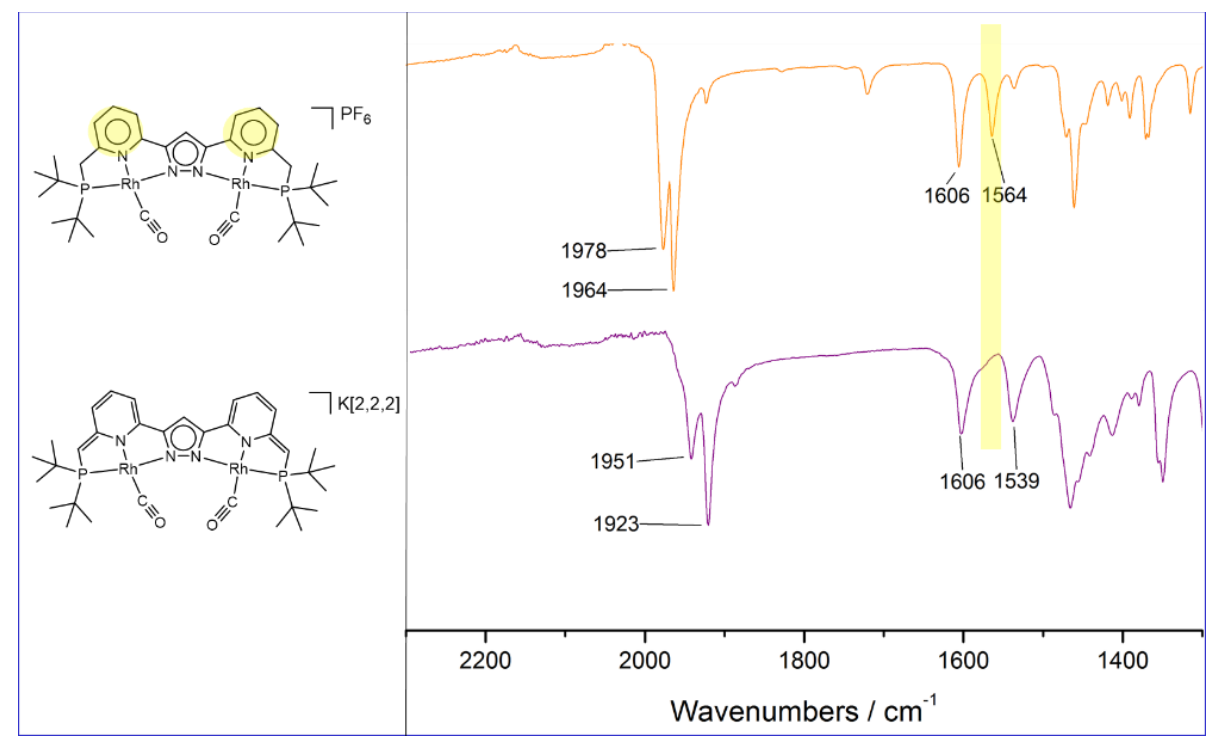

Figure 3.9. ATR-IR of Rh complexes before and after dearomatization. 


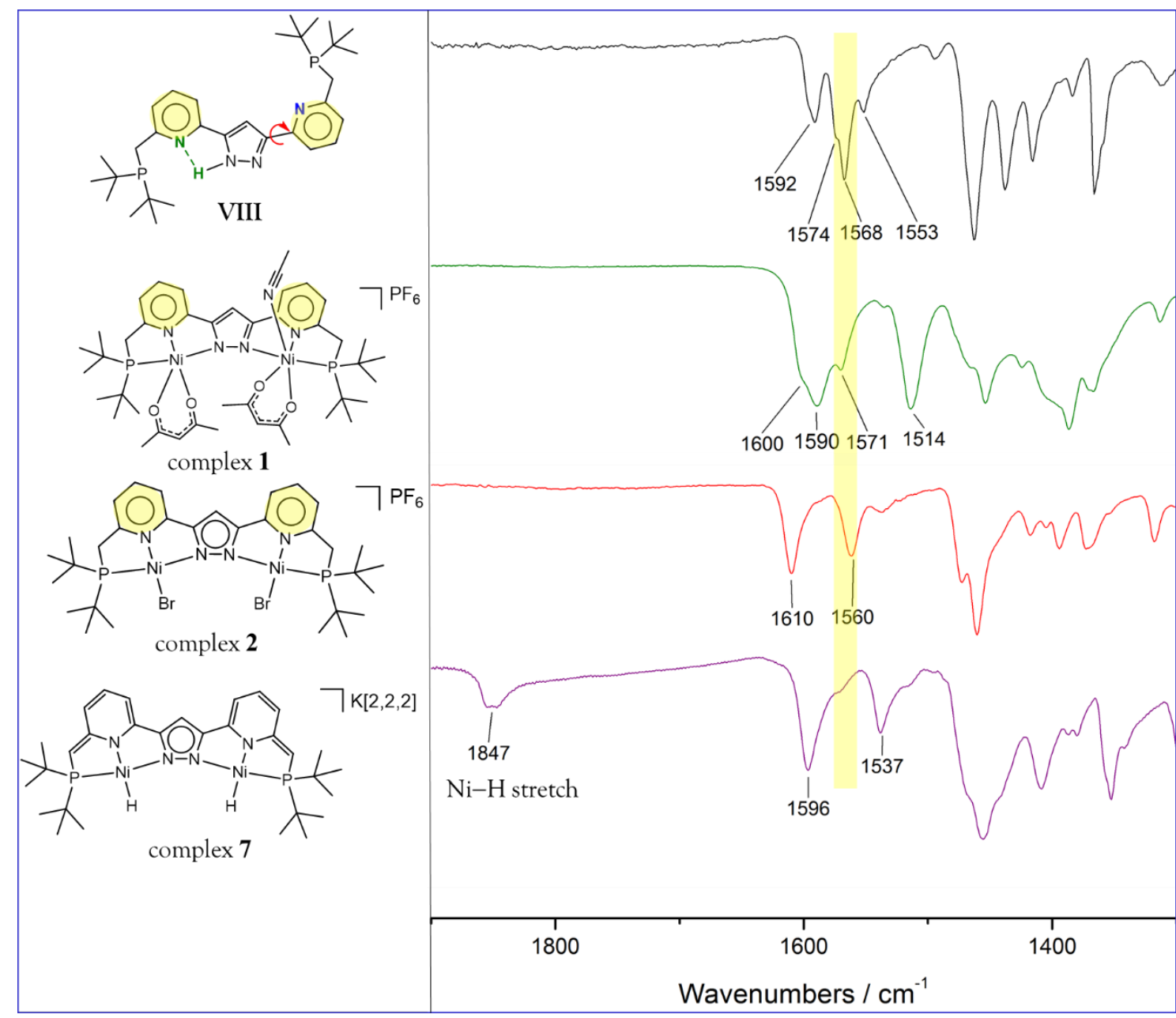

Figure 3.10. ATR-IR of complex 7. The IR spectra of crystalline material provide an indication for the dearomatization of the pyridine.

\subsection{Reactivity with $\mathrm{D}_{2}$ and $\mathrm{H}_{2}$}

Several $\mathrm{Ni}^{\mathrm{II}}$ complexes have been reported to heterolytically split $\mathrm{H}_{2}$ by metal ligand cooperation (MLC) (Figure 3.11). The different mechanisms of dihydrogen splitting have been briefly summarized elsewhere. ${ }^{159}$ The Ni phosphine complex J designed by Dubois in 2009 (Figure 3.11) involved a flexible pendant amine arm which was important in the reaction of dihydrogen oxidation. ${ }^{143}$ In 2010, complex $\mathbf{K}$ synthesized by Caulton involved an amido function with the same reactivity. ${ }^{160}$ Both complexes supposedly oxidized from $\mathrm{Ni}^{\mathrm{II}}$ to a dihydride $\mathrm{Ni}^{\mathrm{IV}} \mathrm{H}_{2}$ intermediate. ${ }^{143,160,161}$ This $\mathrm{Ni}^{\mathrm{IV}} \mathrm{H}_{2}$ intermediate further evolved to the mono-hydride $\mathrm{Ni}^{\mathrm{II}}-\mathrm{H}$ after a proton transfer to a peripheral nitrogen atom. It was recently compared to Noyori's bifunctional mechanism, ${ }^{162}$ where the oxidation of $\mathrm{H}_{2}$ was occurring by the simultaneous separation of $\mathrm{H}_{2}$ into $\mathrm{H}^{-}$and $\mathrm{H}^{+}$. The $\mathrm{H}^{-}$ligand migrated to the metal and the remaining $\mathrm{H}^{+}$reacted with the nucleophilic atom in the outer coordination sphere of the metal. Interestingly Peters proposed in 2012 a nickel-boryl system $\mathbf{L}$ which also activated dihydrogen in a bifunctional manner. ${ }^{163}$ This reactivity was further demonstrated to be an efficient way to hydrogenate olefins. ${ }^{164}$ Since complex 
7 was doubly dearomatized in its isolated form, it represented an excellent candidate to test whether it would activate $\mathrm{H}_{2}$ by MLC (cf Chapter 2).

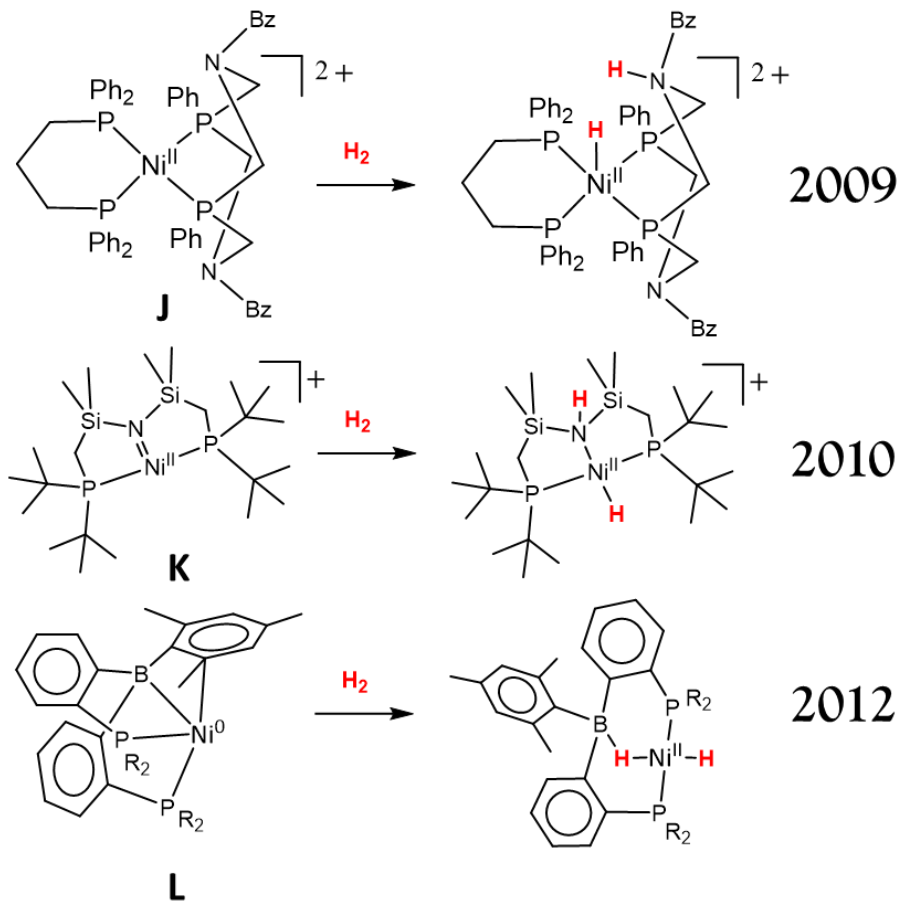

Figure 3.11. $\mathrm{Ni}^{2+}$ pincer systems which can heterolytically split $\mathrm{H}_{2}$ by MLC.

Complex 7 was dissolved in THF- $\mathrm{d}^{8}$ and the solution was transferred in a high-pressure Young NMR tube. The presence of water was avoided by a thorough drying procedure detailed in the experimental section. A pressure of 6 bars of $\mathrm{H}_{2}$ corresponded to more than 50 equivalents of $\mathrm{H}_{2}$ in the following NMR experiments. Thus, $\mathrm{H}_{2}$ was introduced in excess. It is a critical parameter for the qualitative evaluation of the $\mathrm{H} / \mathrm{D}$ exchange.

In the presence of an excess of $\mathrm{D}_{2}, \mathrm{~K}[2,2,2]\left[\mathrm{LNi}_{2}(\mathrm{H})_{2}\right]$ (complex 7) released small amounts of $\mathrm{HD}$ and the hydride resonance located at $-20.20 \mathrm{ppm}$ disappeared (Figure 3.12). The reaction was followed by ${ }^{31} \mathrm{P}$ NMR spectroscopy (Figure 3.14). The singlet for the initial two equivalent phosphorus atoms at $84.34 \mathrm{ppm}$ in $\mathrm{K}[2,2,2]\left[\mathrm{LNi}_{2}(\mathrm{H})_{2}\right]$ changed to a 1:1:1 downfield shifted triplet at $84.61 \mathrm{ppm}$. This suggested that the $\mathrm{Ni}-\mathrm{H}$ moiety in complex 7 was slowly exchanged to $\mathrm{Ni}-\mathrm{D}$ moiety. The appearance of a deuterium close from the phosphorus atom gave rise to a coupling pattern on the ${ }^{31} \mathrm{P}$ NMR spectrum: a triplet with ${ }^{2} \mathrm{~J}_{\mathrm{P}-\mathrm{D}}=14.5 \mathrm{~Hz}$. Thus, $\mathrm{K}[2,2,2]\left[\mathrm{LNi}_{2}(\mathrm{H})_{2}\right]$ was indirectly shown to undergo a sequentially exchange, giving $\mathrm{K}[2,2,2]\left[\mathrm{LNi}_{2}(\mathrm{HD})\right]$ and then $\mathrm{K}[2,2,2]\left[\mathrm{LNi}_{2}(\mathrm{D})_{2}\right]$ as it is represented in Figure 3.13. A concerted elimination of $\mathrm{H}_{2}$ was not consistent with the analysis of the ${ }^{1} \mathrm{H}$ NMR shown in Figure 3.12 (HD was detected but not $\mathrm{H}_{2}$ ). 


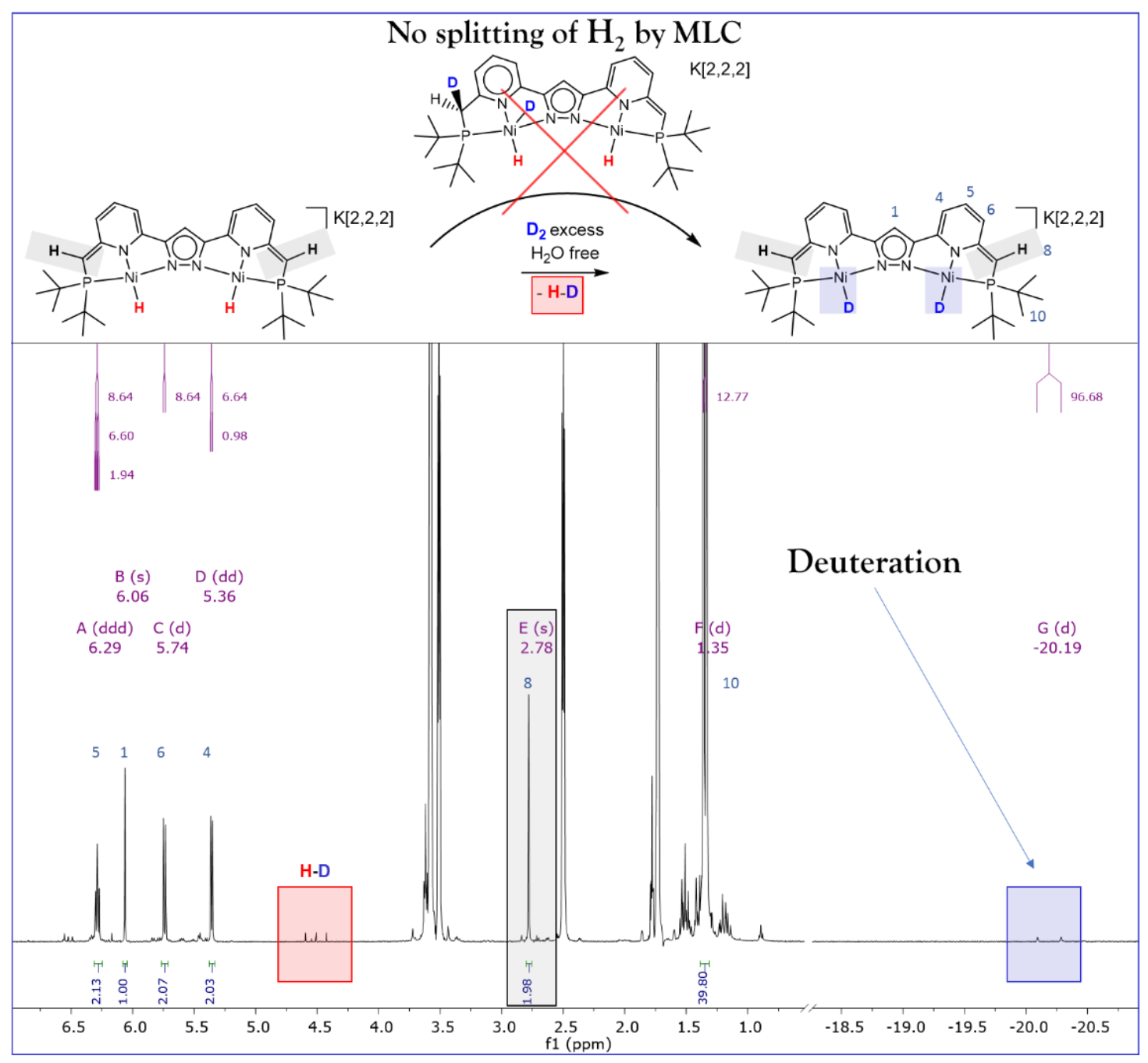

Figure 3.12. In situ ${ }^{1} \mathrm{H}$ NMR spectrum of $\mathrm{K}[2,2,2]\left[\mathrm{LNi}_{2}(\mathrm{D})_{2}\right]$ after $\mathrm{K}[2,2,2]\left[\mathrm{LNi}_{2}(\mathrm{H})_{2}\right]$ quantitatively exchanged with $\mathrm{D}_{2}$. $\mathrm{H}-\mathrm{D}$ was detected during the reaction (red shaded area). The coupling pattern of the C-H group changed but it still integrated for two protons (grey shaded area at $2.78 \mathrm{ppm})$.

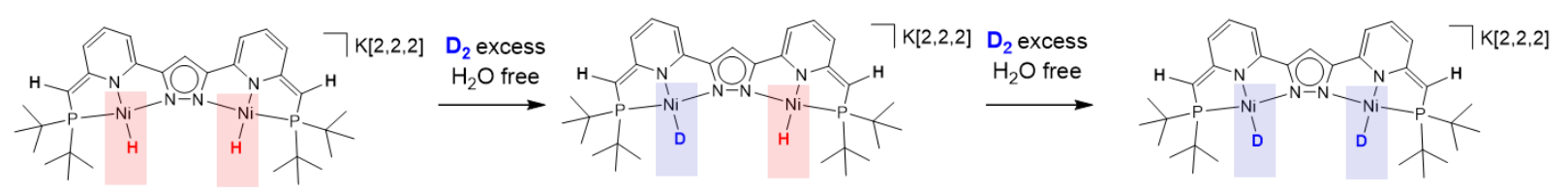

Figure 3.13. Proposed sequential pathway of $H / D$ exchange. The metal ligand cooperation is likely not involved in the process. 


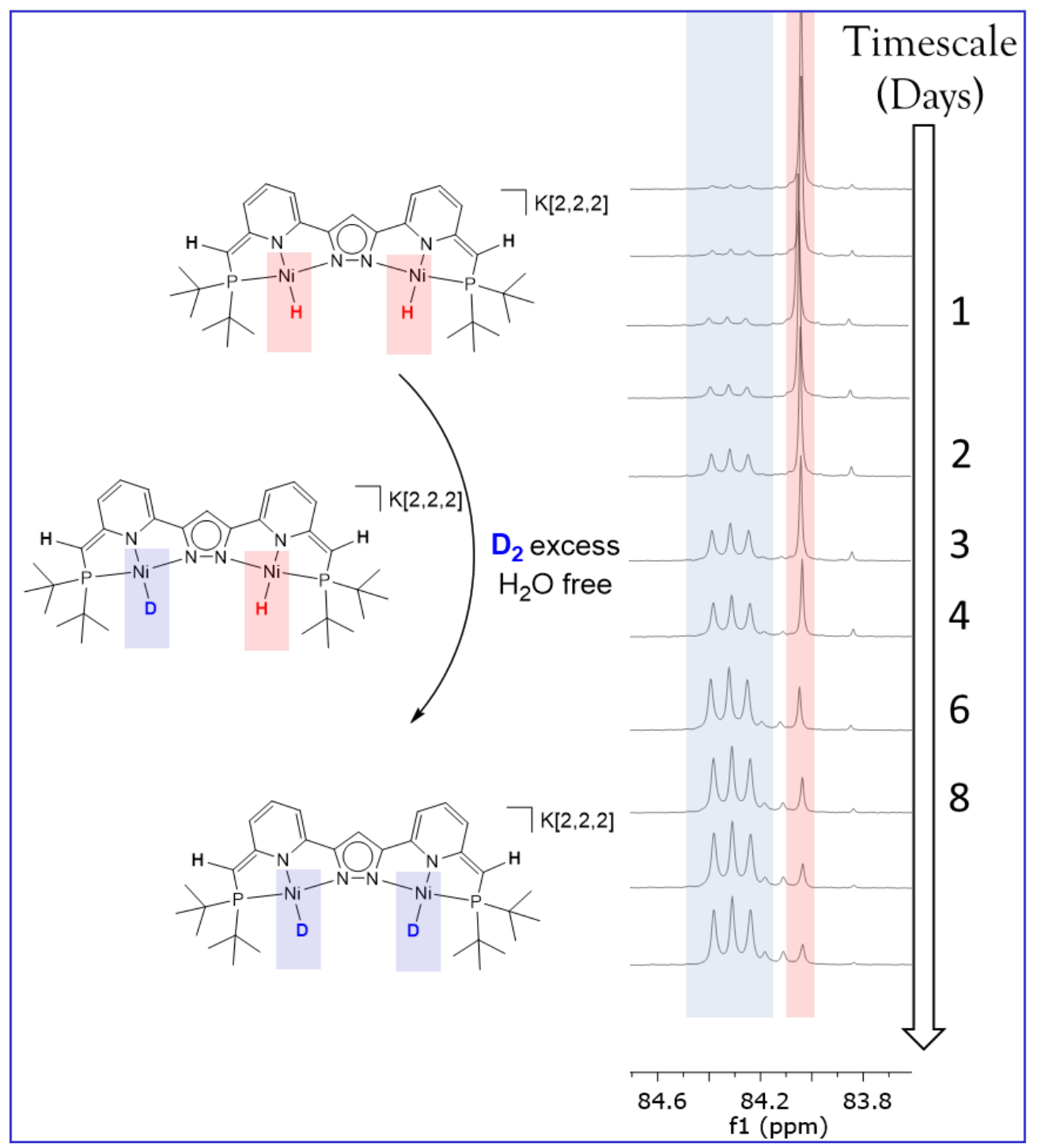

Figure 3.14. Monitoring the reaction of $\mathrm{K}[2,2,2]\left[\mathrm{LNi}_{2}(\mathrm{H})_{2}\right.$ with $\mathrm{D}_{2}$ by ${ }^{31} \mathrm{P} \mathrm{NMR}$ spectroscopy. Formation of Ni-D motif was evidenced by a downfield shifted $1: 1: 1$ triplet of ${ }^{2} \mathrm{~J}_{\mathrm{P}-\mathrm{D}}=14.5 \mathrm{~Hz}$ (blue shaded area)

Surprisingly, if the sample was exposed to $\mathrm{D}_{2}$ for a longer period of time, it led to the appearance of multiple ${ }^{31} \mathrm{P}$ NMR peaks (Figure 3.15, after six days). No shifts and no new signals were observed on the ${ }^{1} \mathrm{H}$ NMR spectrum. However, the intensity of some ${ }^{1} \mathrm{H}$ NMR signals slightly decreased until they disappeared after a period of fourteen days. Then, a single triplet was observed on the ${ }^{31} \mathrm{P}$ NMR spectrum. These observations suggested the further deuteration of the complex $\mathrm{K}[2,2,2]\left[\mathrm{LNi}_{2}(\mathrm{D})_{2}\right]$. 


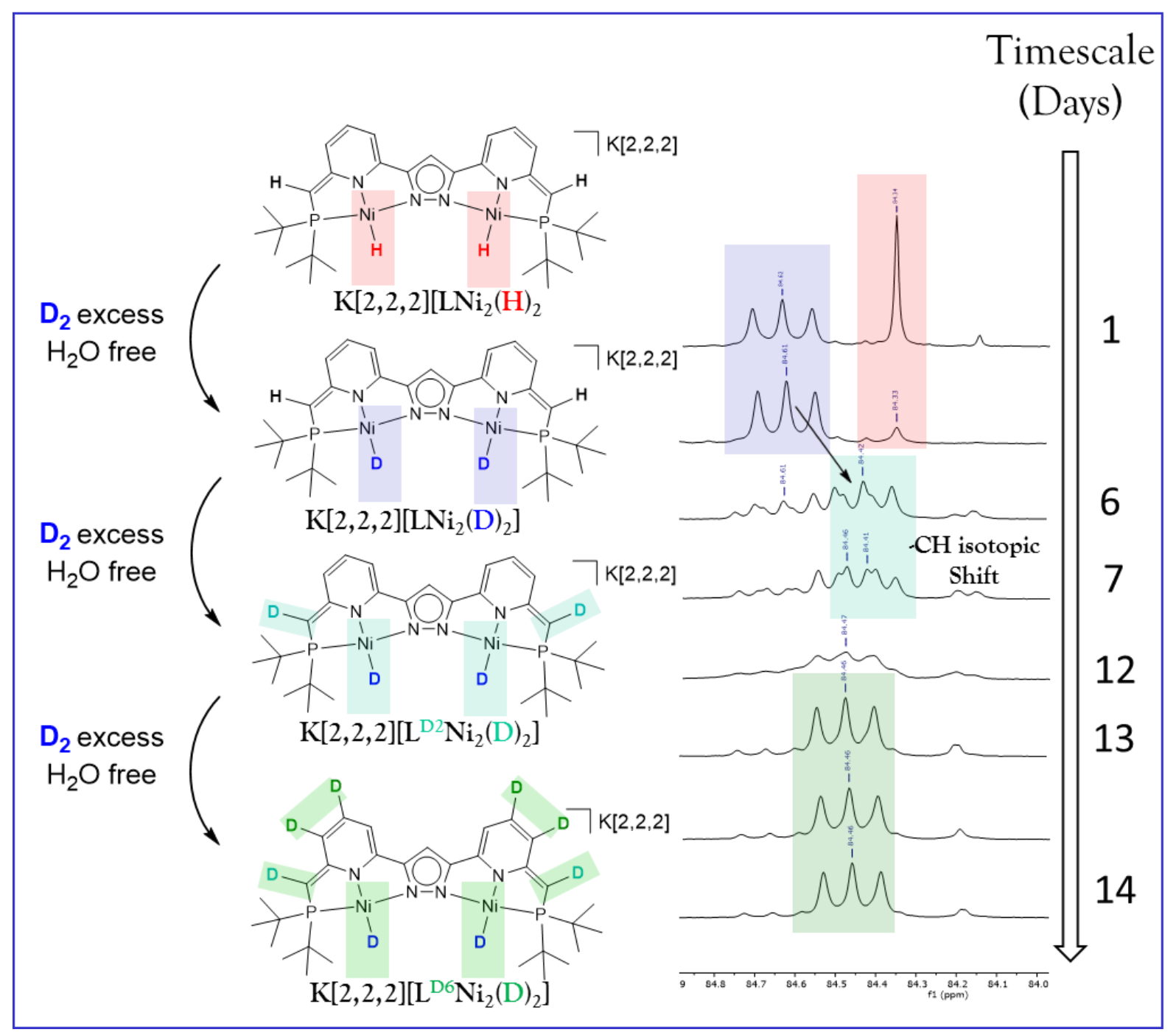

Figure 3.15. Monitoring the deuteration of the aromatic backbone by ${ }^{31} \mathrm{P}$ NMR spectroscopy. A strong shift isotope effect is particularly observed when \{HC-P-Ni-D\} of complex $\mathrm{K}[2,2,2]\left[\mathrm{LNi}_{2}(\mathrm{D})_{2}\right]$ is deuterated to fragment $\{\mathrm{DC}-\mathrm{P}-\mathrm{Ni}-\mathrm{D}\}$ in $\mathrm{K}[2,2,2]\left[\mathrm{L}^{\mathrm{D} 4} \mathrm{Ni}_{2}(\mathrm{D})_{2}\right]-\mathrm{d}^{4}$.

The disappearance of certain NMR signals and the change of coupling pattern associated to the NMR signals suggested deuteration of the backbone was happening. The disappearance of the ${ }^{1} \mathrm{H}$ NMR signals is highlighted in Figure 3.16 by green shaded area for the aromatic region and blue shaded area for the aliphatic region. The doublet of proton 4 (Figure 3.16, top ${ }^{1} \mathrm{H}$ NMR spectrum) resulting from ${ }^{3} \mathrm{~J}_{\mathrm{H}-\mathrm{H}}$ coupling with proton 5 has transformed into a singlet (Figure 3.16, bottom ${ }^{1} \mathrm{H}$ NMR spectrum). This was due to the replacement of the ${ }^{1} \mathrm{H}$ by ${ }^{2} \mathrm{H}$ nuclei in position 5. In the ${ }^{13} \mathrm{C}$ NMR spectra (Figure 3.17), the signals of the carbons bound to deuterium (C-D) appeared to be very broad in contrast to the $\mathrm{C}-\mathrm{H}$ signals. This was due to the coupling between ${ }^{13} \mathrm{C}$ and ${ }^{2} \mathrm{H}$ nuclei. Since the assignment of protons affected by the deuteration was unequivocal, it was possible to propose a structure for the different isotopomers formed during the process. $\mathrm{K}[2,2,2]\left[\mathrm{LNi}_{2}(\mathrm{D})_{2}\right]$ involved two deuterium bound to the metal (deuterides) and a longer exposition with pressure of $\mathrm{D}_{2}$ led to the formation of the subsequent isotopomers: $\mathrm{K}[2,2,2]\left[\mathrm{L}^{\mathrm{D} 2} \mathrm{Ni}_{2}(\mathrm{D})_{2}\right]$ and then $\mathrm{K}[2,2,2]\left[\mathrm{L}^{\mathrm{D} 6} \mathrm{Ni}_{2}(\mathrm{D})_{2}\right] .{ }^{31} \mathrm{P}$ NMR spectroscopy was a critical method employed for the interpretation of deuteration of the aromatic backbone since strong secondary 
$\mathrm{H} / \mathrm{D}$ shift effects were involved. Particularly, if the fragment $\{$ HC-P-Ni-D $\}$ of complex $\mathrm{K}[2,2,2]\left[\mathrm{LNi}_{2}(\mathrm{D})_{2}\right]$ was deuterated to give the fragment $\{\mathrm{DC}-\mathrm{P}-\mathrm{Ni}-\mathrm{D}\}$ in $\mathrm{K}[2,2,2]\left[\mathrm{L}^{\mathrm{D} 2} \mathrm{Ni}_{2}(\mathrm{D})_{2}\right]$, an upfield shift of $0.25 \mathrm{ppm}$ was observed (Figure 3.15). ${ }^{1} \mathrm{H}$ and ${ }^{13} \mathrm{C}$ NMR spectra of $\mathrm{K}[2,2,2]\left[\mathrm{LNi}_{2}(\mathrm{H})_{2}\right]$ and $\mathrm{K}[2,2,2]\left[\mathrm{L}^{\mathrm{D} 6} \mathrm{Ni}_{2}(\mathrm{D})_{2}\right]$ are directly compared in Figure 3.16 and Figure 3.17 respectively. To finally confirm the existence of $\mathrm{K}[2,2,2]\left[\mathrm{L}^{\mathrm{D} 6} \mathrm{Ni}_{2}(\mathrm{D})_{2}\right]$, the complex was dissolved in non-deuterated THF. A ${ }^{2} \mathrm{H}$ NMR experiment was recorded. Signals in the aromatic and aliphatic regions could be detected and integrated (Figure 3.18). The Ni-D resonance appeared as a doublet at $-20.20 \mathrm{ppm}$ with ${ }^{2} \mathrm{~J}_{\mathrm{D}-\mathrm{P}}=14.5 \mathrm{~Hz}$, perfectly in line with the triplet signal observed in the ${ }^{31} \mathrm{P}$ NMR spectrum (Figure 3.15).

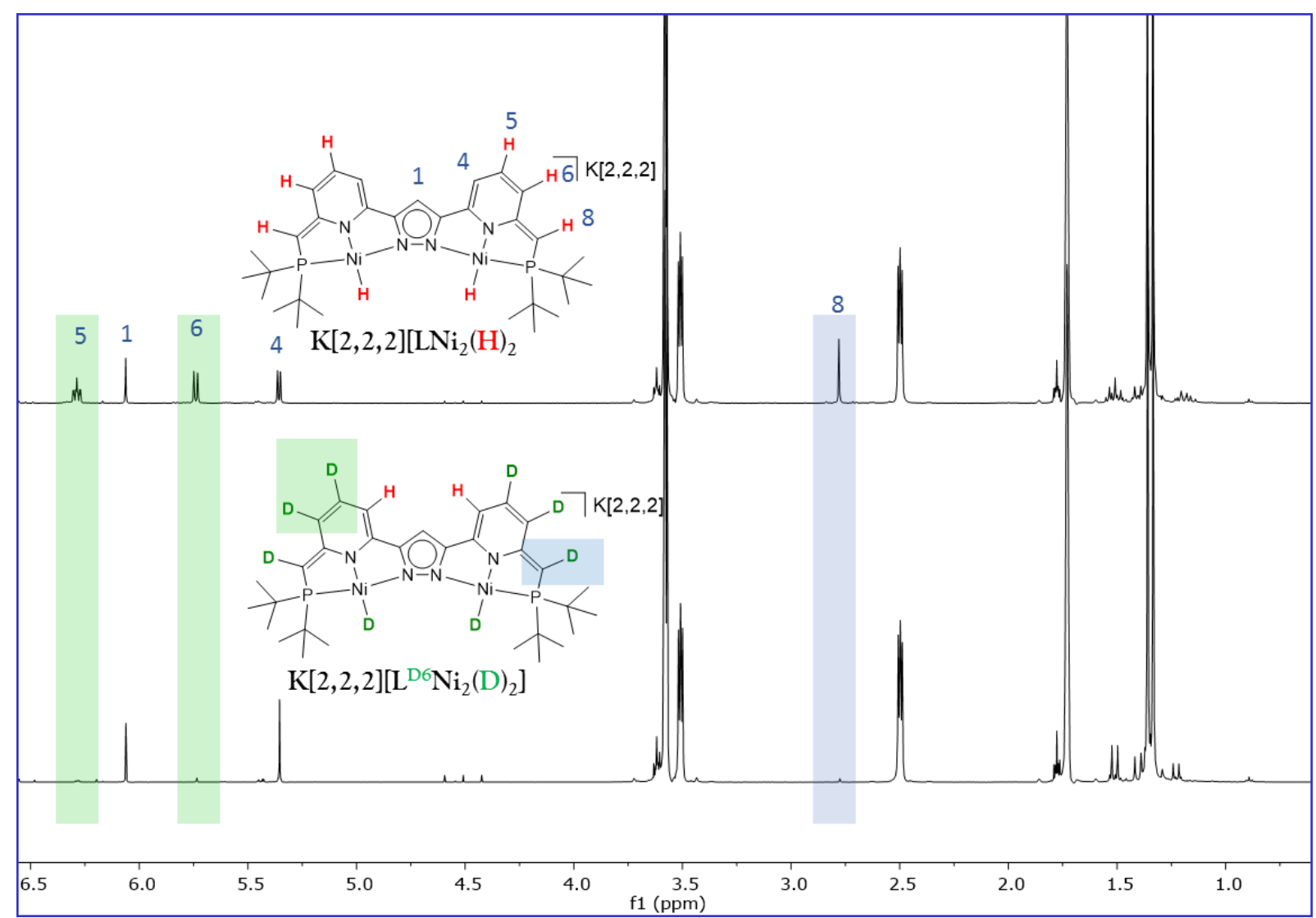

Figure 3.16. Comparison of ${ }^{1} \mathrm{H} \quad \mathrm{NMR}$ spectra of $\mathrm{K}[2,2,2]\left[\mathrm{LNi}_{2}(\mathrm{H})_{2}\right] \quad$ (top) and $\mathrm{K}[2,2,2]\left[\mathrm{L}^{\mathrm{D} 6} \mathrm{Ni}_{2}(\mathrm{D})_{2}\right]$ (bottom). in THF-d ${ }^{8}$ 


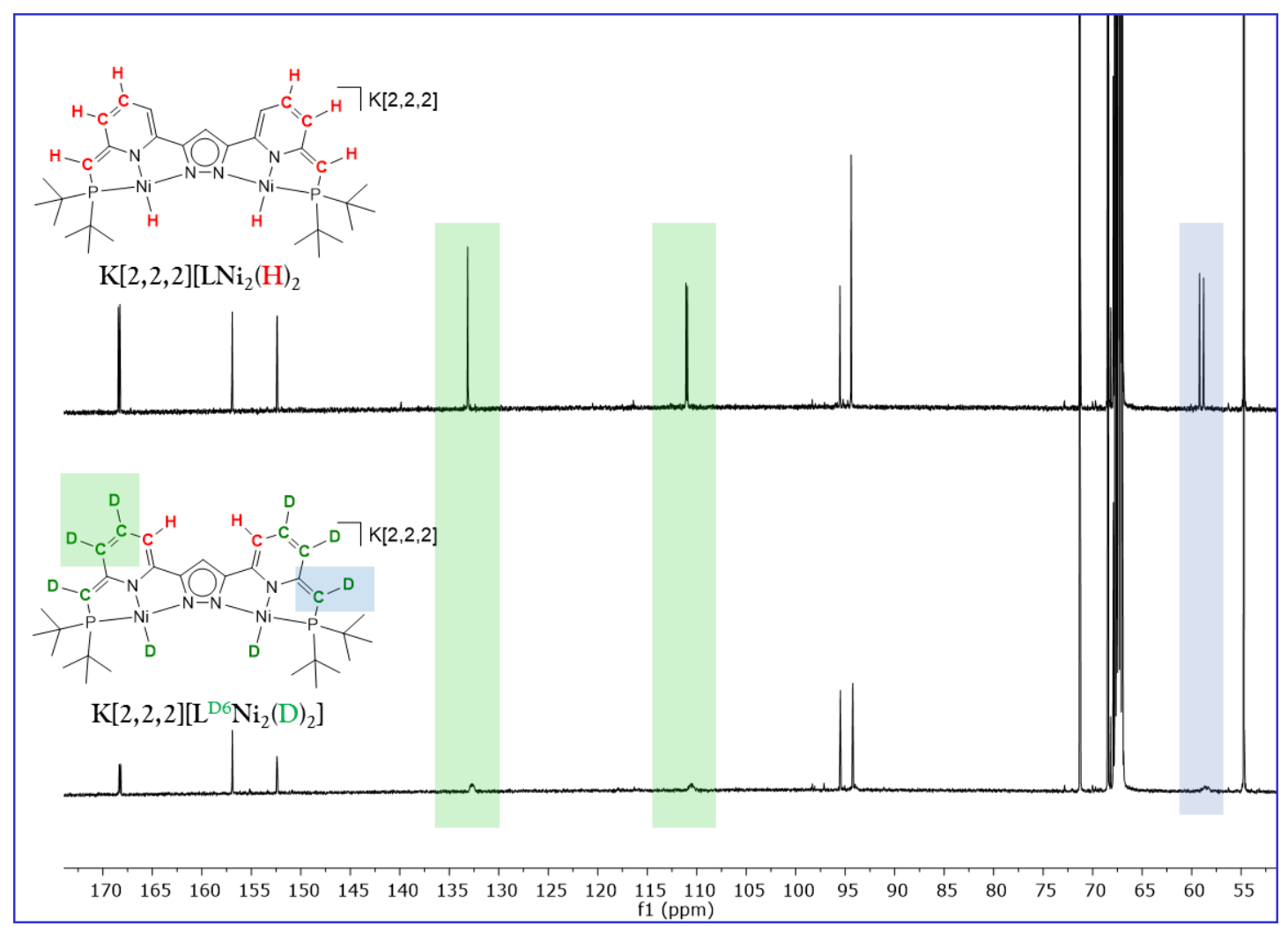

Figure 3.17. Comparison of ${ }^{13} \mathrm{C} \quad \mathrm{NMR}$ spectra of $\mathrm{K}[2,2,2]\left[\mathrm{LNi}_{2}(\mathrm{H})_{2}\right.$ (top) and $\mathrm{K}[2,2,2]\left[\mathrm{L}^{\mathrm{D} 6} \mathrm{Ni}_{2}(\mathrm{D})_{2}\right]$ (bottom) in THF-d ${ }^{8}$

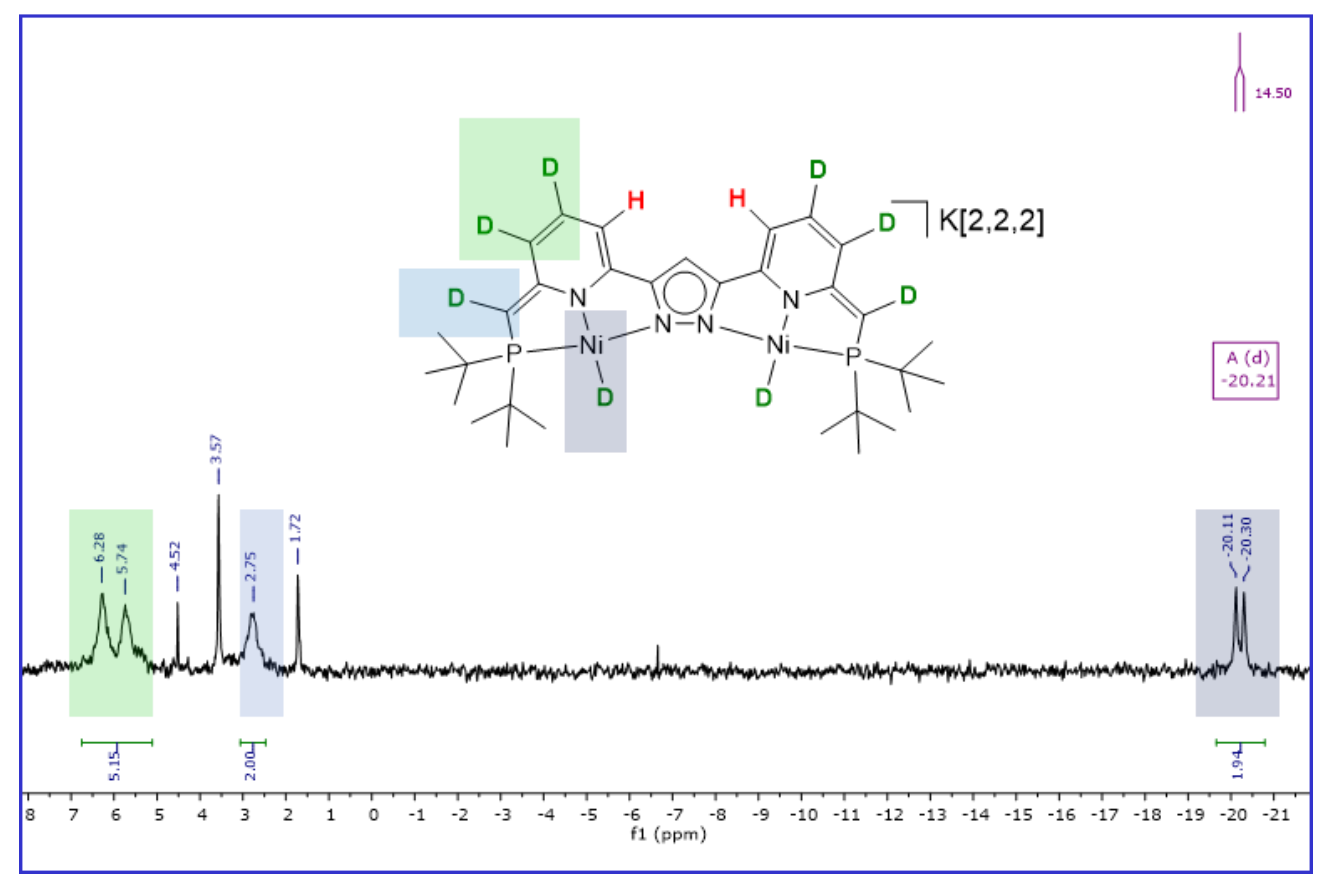

Figure 3.18. ${ }^{2} \mathrm{H}$ NMR of $\mathrm{K}[2,2,2]\left[\mathrm{L}^{\mathrm{D} 6} \mathrm{Ni}_{2}(\mathrm{D})_{2}\right]$ in $\mathrm{THF}-\mathrm{h}^{8}$ ( not deuterated).

The reproducibility of those reactions in view to conduct kinetic experiments and to obtain additional mechanistic information was hampered by the highly moisture sensitive character of complex 7. Indeed, traces of water readily reacted with the complex according to the processes 
described in Chapter 4. The hydrolysed complexes displayed a characteristic reactivity towards $\mathrm{H}_{2}$ which was different from complex 7. Thus, if water was detected in the initial step of $\mathrm{D}_{2}$ or $\mathrm{H}_{2}$ addition, the NMR sample was discarded as it could not give reliable information. Indeed, the presence of several complexes with different reactivities in an NMR sample would not give reliable information for the qualitative analysis of dihydrogen activation.

$\mathrm{K}[2,2,2]\left[\mathrm{LNi}_{2}(\mathrm{H})_{2}\right]$ was reminiscent of the dinickel dihydride complex reported by D. Manz, as it also contained two terminal hydrides within the bimetallic pocket of two $\mathrm{Ni}^{2+}$ ions. ${ }^{165}$ However, the reactivity of the $\mathrm{Ni}-\mathrm{H}$ bond toward $\mathrm{H}_{2} / \mathrm{D}_{2}$ was different from that of 7 .

While the system developed by Manz was reported to eliminate the two hydrides pairwise as $\mathrm{H}_{2} / \mathrm{D}_{2}$ by reductive elimination without formation of $\mathrm{HD},{ }^{154}$ the complex 7 (developed in this chapter) exchanged $\mathrm{D}_{2}$ with $\mathrm{HD}$ sequentially (Figure 3.19). The $\mathrm{Ni} \cdots \mathrm{Ni}$ distance was similar in both systems (4.158 A in Manz system and 4.156 $\AA$ in complex 7). Thus, the intermetallic distance was not be the critical parameter in the reactivity difference observed between the two systems, whilst the electronic and sterical configurations from surrounding ligands were significantly different. An important concluding remark was that $\mathrm{K}[2,2,2]\left[\mathrm{LNi}_{2}(\mathrm{H})_{2}\right]$ likely did not heterolytically split $\mathrm{H}_{2}$ by metal ligand cooperation as the simultaneous deuteration of \{HC-P-NiH\} to $\{$ DC-P-Ni-D $\}$ was not observed.

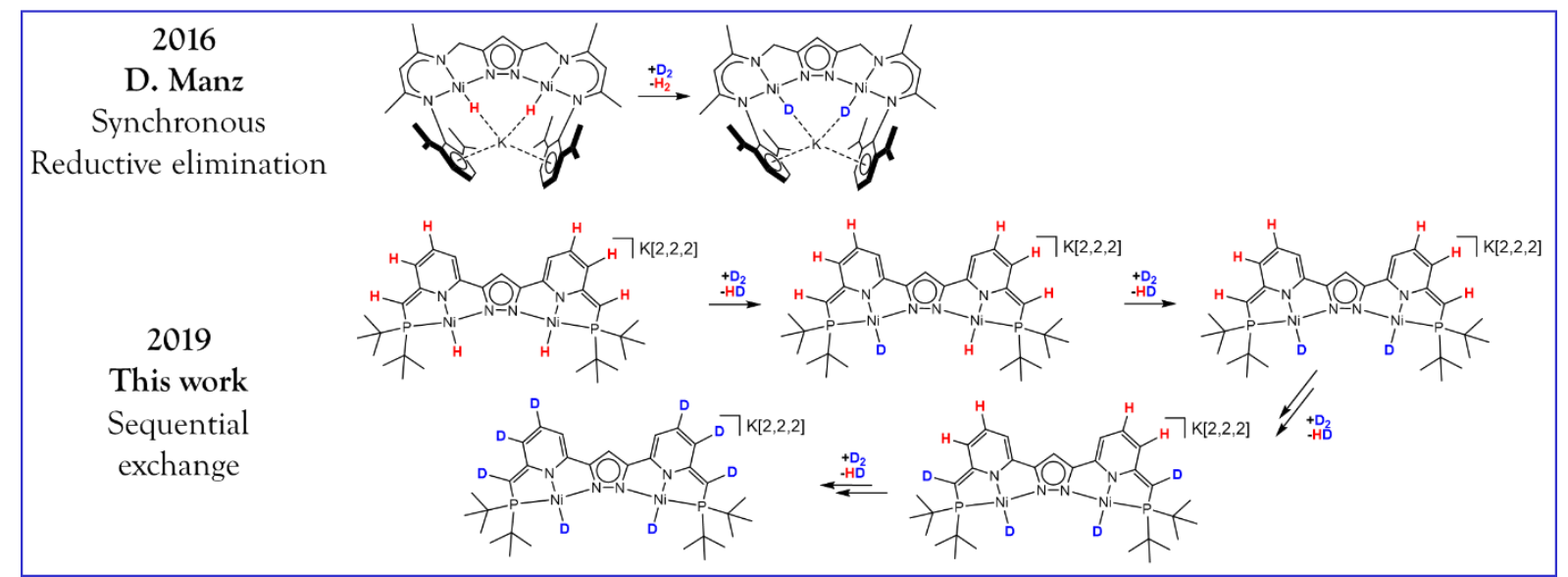

Figure 3.19. Different pathways of dihydrogen activation in dinickel dihydride pyrazolate based complexes.

\subsection{Reactivity with ethylene}

When heating in presence of ethylene $\left(\mathrm{C}_{2} \mathrm{H}_{4}\right), \mathrm{K}[2,2,2]\left[\mathrm{LNi}_{2}(\mathrm{H})_{2}\right](7)$ slowly reacted to give a new complex which was characterized by X-ray diffraction: $\mathrm{K}[2,2,2]\left[\mathrm{LNi}\left(\mu-\mathrm{C}_{2} \mathrm{H}_{4}\right) \mathrm{Ni}\right](8)$ (Figure 3.20). The double bond of ethylene has been reduced to give a $\mathrm{C}_{2} \mathrm{H}_{4}{ }^{2-}$ ethane-1,2-diyl ligand which was stabilized by the two metals. Indeed, the $\mathrm{C}-\mathrm{C}$ bond length is $1.553 \AA$ and the geometry of both carbon atoms is close to tetrahedral indicating $\mathrm{sp}^{3}$ carbons, which was in line with the reduction of the $\mathrm{C}=\mathrm{C}$ bond. $\mathrm{A}_{2} \mathrm{H}_{4}{ }^{2-}$ ethane-1,2-diyl ligand has already been observed once in a dinickel compound. ${ }^{166}$ 

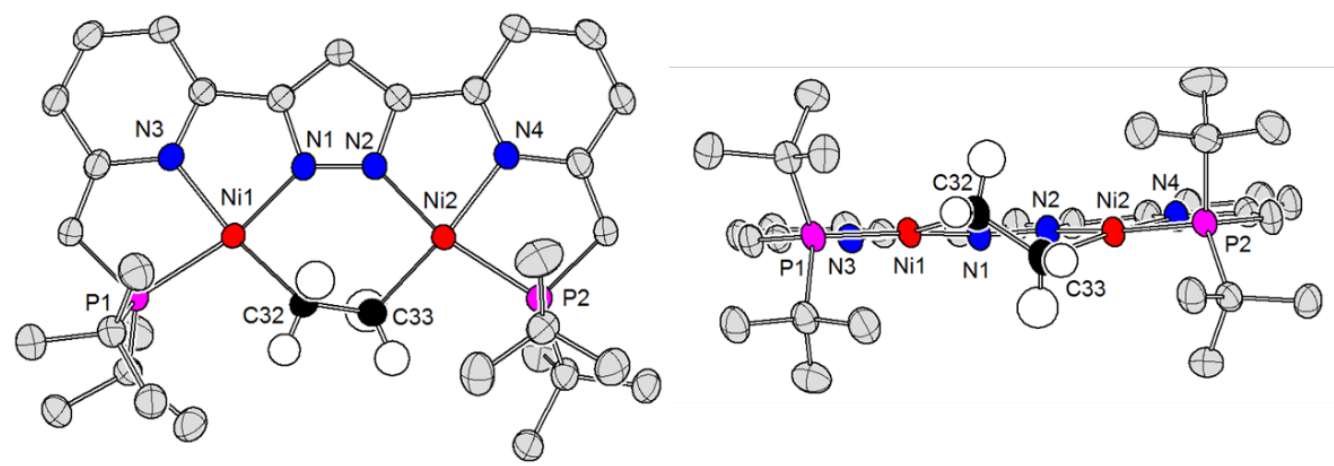

Figure 3.20 Molecular structure (thermal displacement ellipsoids shown at $50 \%$ probability) of the anion of complex $\mathbf{8}$. Most of the hydrogen atoms and all cations have been omitted for clarity. Left: top view of the molecular structure. Right: front view of the molecular structure

\begin{tabular}{cc|cc}
\hline \multicolumn{3}{l|}{ Bond Lengths around $\mathrm{Ni}(1) / \AA$} & Bond Lengths around $\mathrm{Ni}(2) / \AA$ \\
\hline \multicolumn{2}{l}{} & & \\
\hline $\mathrm{Ni}(1)-\mathrm{N}(1)$ & $1.8840(19)$ & $\mathrm{Ni}(2)-\mathrm{N}(2)$ & $1.8749(19)$ \\
\hline $\mathrm{Ni}(1)-\mathrm{N}(3)$ & $1.9326(19)$ & $\mathrm{Ni}(2)-\mathrm{N}(4)$ & $1.9317(19)$ \\
\hline $\mathrm{Ni}(1)-\mathrm{C}(32)$ & $1.946(2)$ & $\mathrm{Ni}(2)-\mathrm{C}(33)$ & $1.948(2)$ \\
\hline $\mathrm{Ni}(1)-\mathrm{P}(1)$ & $2.1645(6)$ & $\mathrm{Ni}(2)-\mathrm{P}(2)$ & $2.1633(6)$ \\
\hline $\mathrm{C}(32)-\mathrm{C}(33)$ & $1.553(4)$ & \multicolumn{2}{l}{}
\end{tabular}

Table 3.2. Selected bond lengths

The doubly dearomatized state of complex $\mathbf{8}$ has been confirmed by a metrical analysis of the Xray data for the pyridine moieties. The alternating $\mathrm{C}=\mathrm{C}$ and $\mathrm{C}-\mathrm{C}$ bonds were represented in Figure 3.21. Interestingly, the $\mathrm{Ni}(1)-\mathrm{P}(1)$ is about $0.042 \AA$ longer in complex $\mathbf{8}$ than in complex 7. $\mathrm{Ni}(1)-\mathrm{N}(3)$ was also extended by about $0.030 \AA$. The elongation of those coordination bonds suggested a stronger electronic donation from the $\mathrm{C}_{2} \mathrm{H}_{4}{ }^{2-}$ ligand than from the hydride in complex 7.

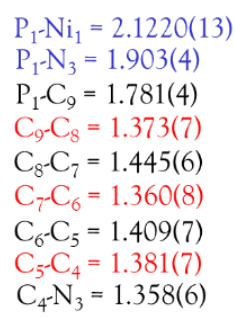

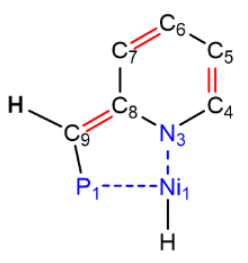

$\mathrm{K}[2,2,2]\left[\mathrm{LNi}_{2}(\mathrm{H})_{2}\right]$ complex 7

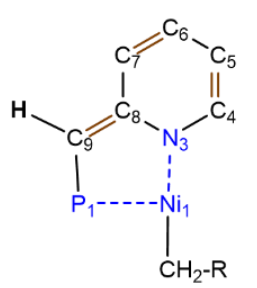

$\mathrm{P}_{1}-\mathrm{Ni}_{1}=2.1645(6)$

$\mathrm{P}_{1}-\mathrm{N}_{3}=1.9326(19)$

$\mathrm{P}_{1}-\mathrm{C}_{9}=1.772(2)$

$\mathrm{C}_{9}-\mathrm{C}_{8}=1.385(3)$

$\mathrm{C}_{8}-\mathrm{C}_{7}=1.437(3)$

$\mathrm{C}_{7}-\mathrm{C}_{6}=1.363(4)$

$\mathrm{C}_{6}-\mathrm{C}_{5}=1.415(3)$

$\mathrm{C}_{5}-\mathrm{C}_{4}=1.371(3)$

$\mathrm{C}_{4}-\mathrm{N}_{3}=1.361(3)$

$\mathrm{K}[2,2,2]\left[\mathrm{LNi}\left(\mu-\mathrm{C}_{2} \mathrm{H}_{4}\right) \mathrm{Ni}\right]$

complex 8

Figure 3.21. Metrical comparison of coordination bonds between complex $\mathbf{7}$ and complex $\mathbf{8}$.

Complex 8 was sensitive to water. Dissolving complex 8 in THF-d ${ }^{8}$ gave rise to different ${ }^{31} \mathrm{P}$ NMR signals probably resulting from the decomposition of the complex. In Figure 3.22 one major peak at $57.1 \mathrm{ppm}$ was observed for complex $\mathbf{8}$ and two other peaks at 68.08 and $57.95 \mathrm{ppm}$ with equal intensity were observed. A pure sample of compound $\mathbf{8}$ could not be obtained as those other peaks always formed, though in variable intensity. The ${ }^{1} \mathrm{H}$ NMR spectrum of this complex was complicated to interpret as the signals for the complex in the aromatic region were unusually 
broad at room temperature which also limited the utilization of two-dimensional NMR methods for the characterization of this complex.

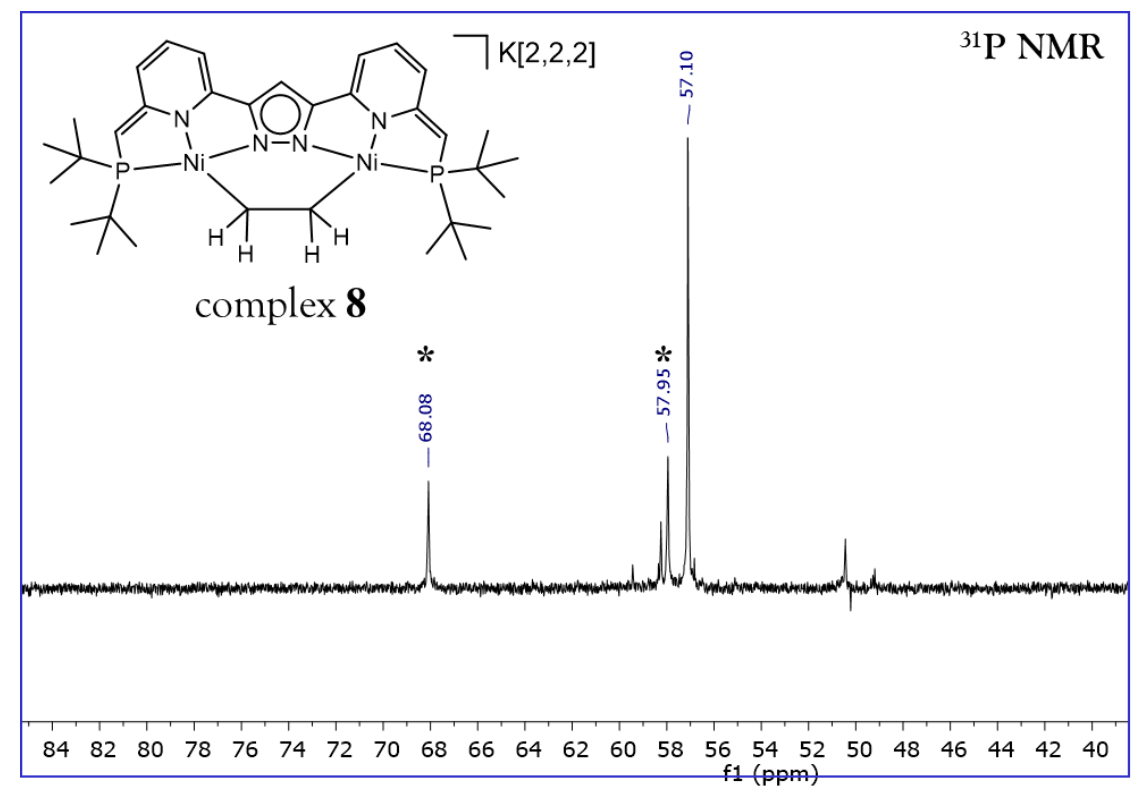

Figure $3.22 .{ }^{31} \mathrm{P}$ NMR spectrum of complex $\mathbf{8}$ in THF- ${ }^{8} .\left(^{*}\right)$ indicates the presence of hydrolysed complex.

A series of relatively clean NMR spectra of complex $\mathbf{8}$ were obtained when ethylene- $\mathrm{d}^{4}$ was used for the synthesis of the complex. Indeed, complex 7 reacted with $\mathrm{CD}_{2}=\mathrm{CD}_{2}$ in order to give a deuterated version of complex $\mathbf{8}, \mathrm{K}[2,2,2]\left[\mathrm{LNi}\left(\mu-\mathrm{C}_{2} \mathrm{D}_{4}\right) \mathrm{Ni}\right]$. The complex was crystalized and then redissolved for NMR measurement in non-deuterated THF. At $298 \mathrm{~K}$, the ${ }^{31} \mathrm{P}$ NMR signal of the complex was low in intensity. Decreasing the temperature to $283 \mathrm{~K}$ led to an increase in signal intensity. The ${ }^{31} \mathrm{P}$ NMR spectrum of this complex in not-deuterated THF is shown in Figure 3.23 and revealed two major peaks at 57.05 and 56.86 ppm. Peaks at 68.20 and 57.89 ppm were also observed and were consistent with the decomposition product of complex $\mathbf{8}$ seen in Figure 3.22. However, the intensity of those ${ }^{31} \mathrm{P}$ NMR signals were much lower compared to the one seen in Figure 3.23. Probably, normal THF solvent used for this measurement contained less water than the THF-d ${ }^{8}$ previously used. A ${ }^{2} \mathrm{H}$ NMR spectrum of $\mathrm{K}[2,2,2]\left[\mathrm{LNi}\left(\mu-\mathrm{C}_{2} \mathrm{D}_{4}\right) \mathrm{Ni}\right]$ did not reveal any signals at room temperature except for the residual amount of deuterated THF. Decreasing the temperature of the NMR sample to $283 \mathrm{~K}$ gave rise to a single broad signal at $0.86 \mathrm{ppm}$ (Figure 3.24). No other signals in a range of 20 to $-20 \mathrm{ppm}$ were observed. This signal observed on the ${ }^{2} \mathrm{H}$ NMR spectrum could correspond to the $\mathrm{C}_{2} \mathrm{D}_{4}{ }^{2-}$ ligand contained in the complex. Indeed, the crystal structure of complex $\mathbf{8}$ indicated the reduction of the ethylene double bond. Thus, the protons of the resulting ethan-1,2-diyl should be found in the aliphatic region of the ${ }^{1} \mathrm{H}$ or ${ }^{2} \mathrm{H}$ NMR resonance. It was confirmed by the spectrum shown in Figure 3.24. 


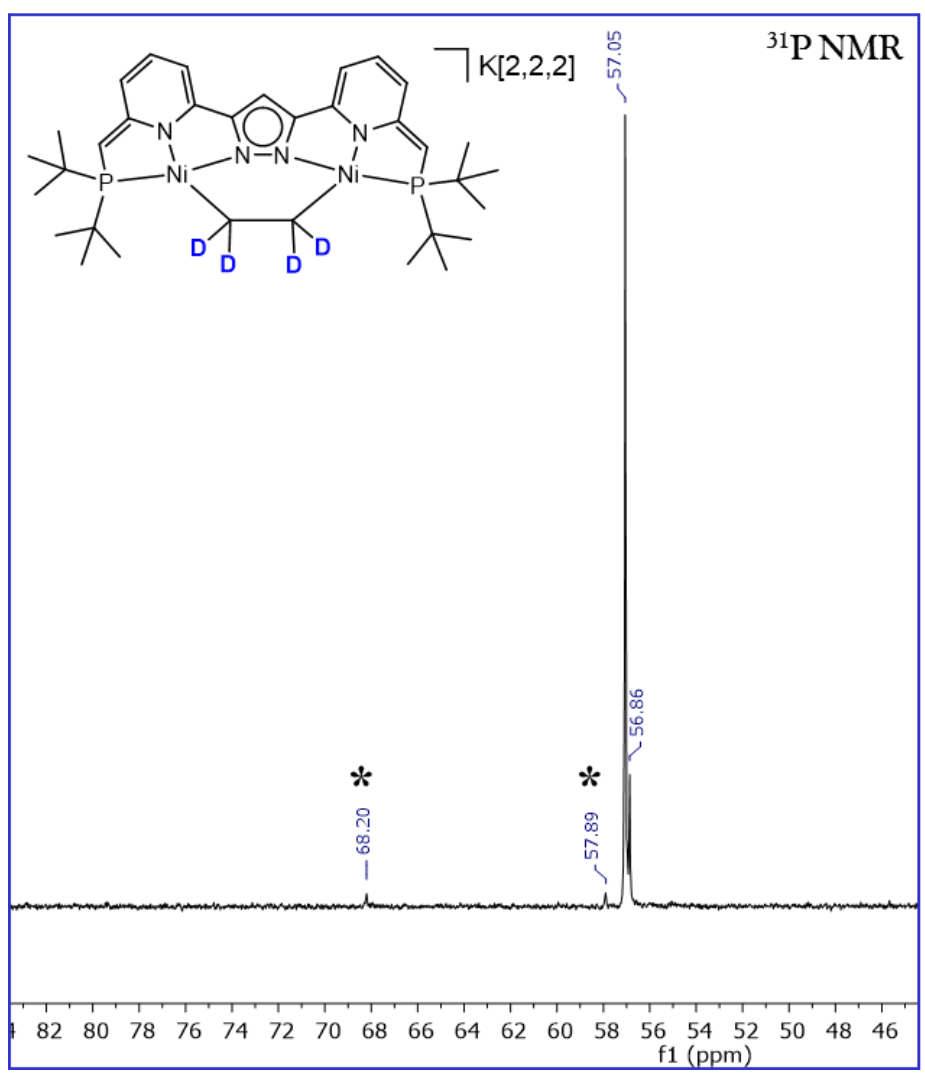

Figure 3.23. ${ }^{31} \mathrm{P}$ NMR spectrum of $\mathrm{K}[2,2,2]\left[\mathrm{LNi}\left(\mu-\mathrm{C}_{2} \mathrm{D}_{4}\right) \mathrm{Ni}\right]$ in THF at $\left.283 \mathrm{~K} .{ }^{*}\right)$ indicates the presence of hydrolysed complex.

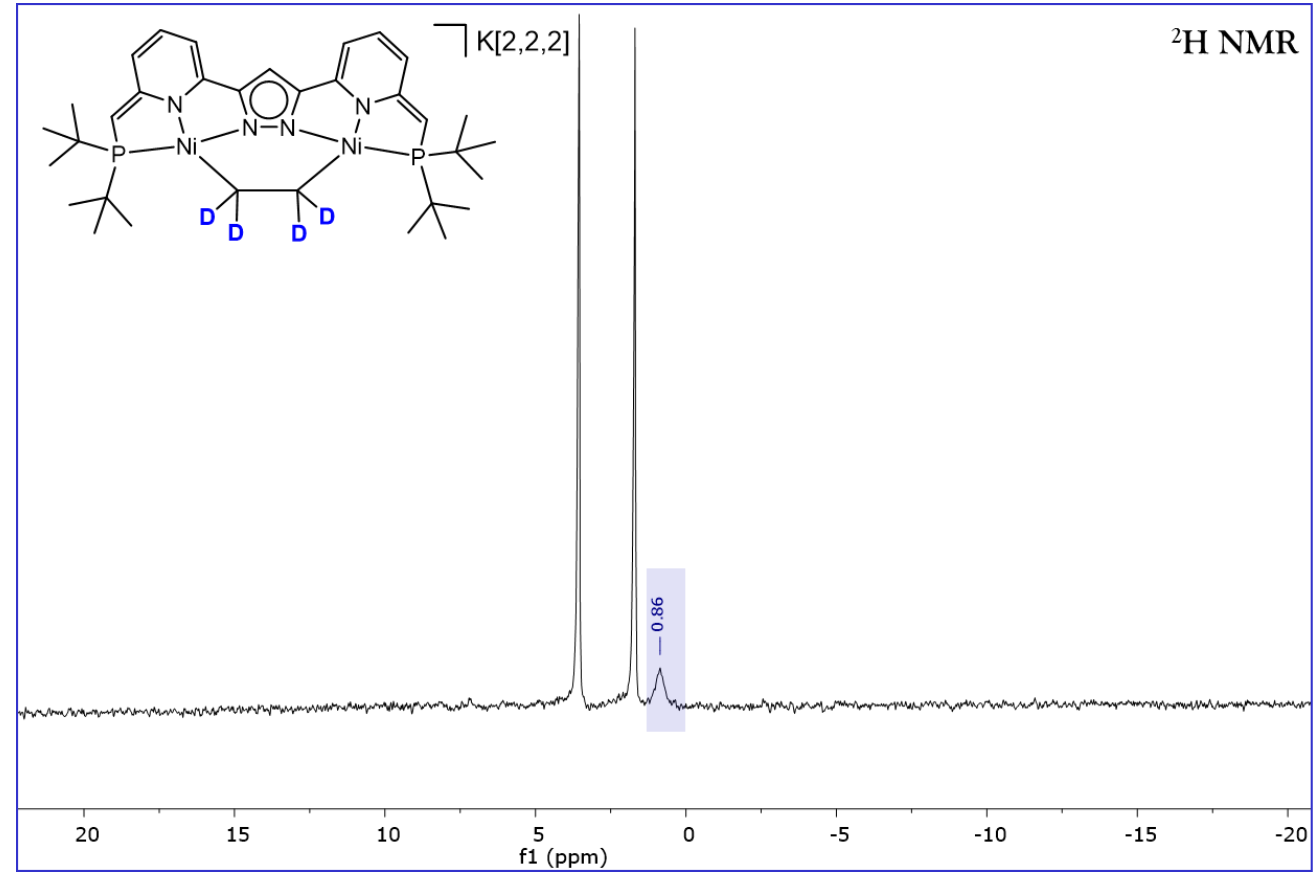

Figure 3.24. ${ }^{2} \mathrm{H}$ NMR spectrum of complex $\mathrm{K}[2,2,2]\left[\mathrm{LNi}\left(\mu-\mathrm{C}_{2} \mathrm{D}_{4}\right) \mathrm{Ni}\right]$ in $\mathrm{THF}$ at $283 \mathrm{~K}$.

The ${ }^{2} \mathrm{H}$ and ${ }^{31} \mathrm{P}$ NMR spectra showed a dependence between signal resolution and temperature. Decreasing the temperature led to better signal quality. In order to confirm this feature, ${ }^{1} \mathrm{H}$ NMR spectra of complex $\mathrm{K}[2,2,2]\left[\mathrm{LNi}\left(\mu-\mathrm{C}_{2} \mathrm{D}_{4}\right) \mathrm{Ni}\right]$ were recorded at different temperatures (Figure 
3.25). At $298 \mathrm{~K}$ the signals of the aromatic backbone of the complex were broad. Increasing the temperature to $323 \mathrm{~K}$ did not lead to any improvement of the NMR spectra. However, when the temperature of the NMR sample was decreased to $283 \mathrm{~K}$, the broadness of the signal decreased and gave rise to a coupling pattern, which was similar to those of dearomatized complexes previously studied in complex $\mathbf{5}, \mathbf{5}$, and $\mathbf{7}$. A ${ }^{13} \mathrm{C}$ NMR spectrum of that complex was recorded at $283 \mathrm{~K}$ and allowed to measure an HSQC (cf supporting informations).

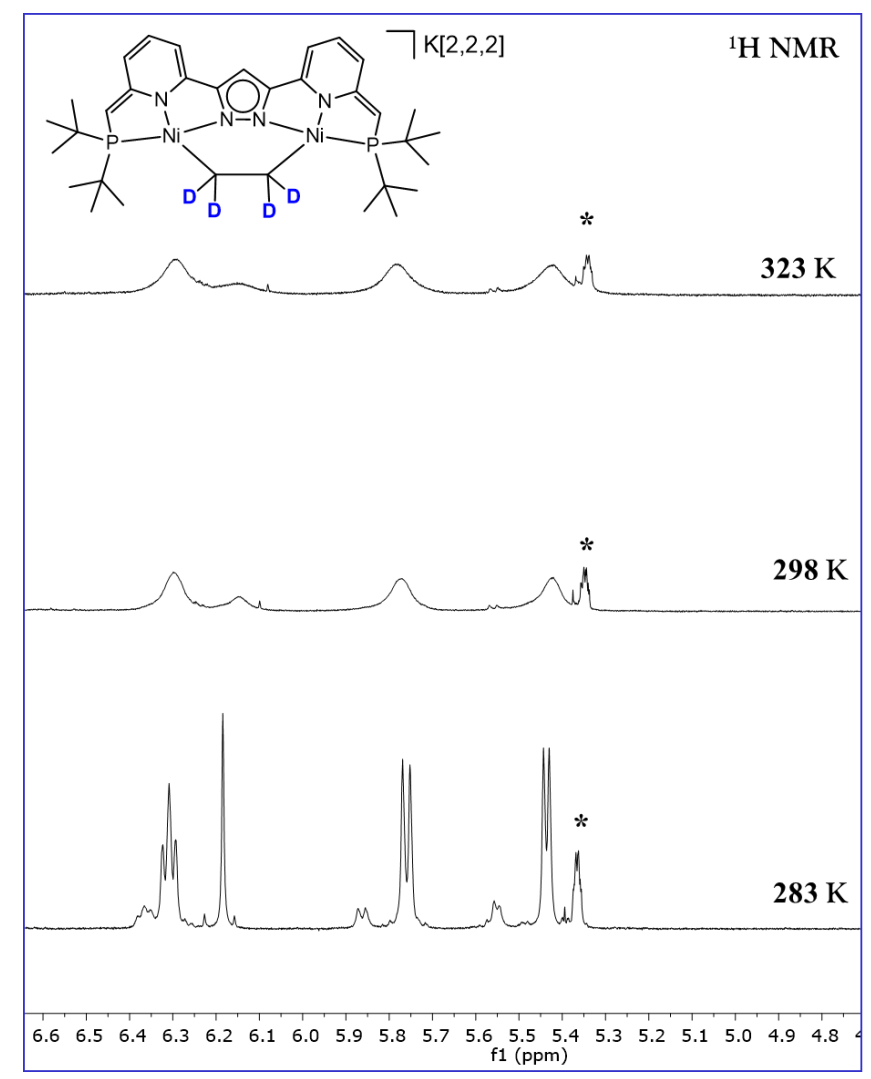

Figure 3.25. ${ }^{1} \mathrm{H}$ NMR spectra of complex $\mathrm{K}[2,2,2]\left[\mathrm{LNi}\left(\mu-\mathrm{C}_{2} \mathrm{D}_{4}\right) \mathrm{Ni}\right]$ in THF-d ${ }^{8}$ at variable temperatures. $\left(^{*}\right)$ likely indicates the presence of $\mathrm{CD}_{2}=\mathrm{CDH}$.

An attempt to clarify the mechanistic pathway of the reaction by monitoring the reaction in situ by NMR spectroscopy was performed. ${ }^{31} \mathrm{P}$ NMR spectra recorded during the reaction of complex 7 to complex 8 in the presence of $\mathrm{CD}_{2}=\mathrm{CD}_{2}$ are shown in Figure 3.26. The addition of absolutely dry ethylene was unfortunately not possible as signals corresponding to a hydrolyzed complex 7 were observed (black asterisks on Figure 3.26). The hydrolysis of complex 7 is studied in detail in the next chapter. Even if a part of complex $\mathbf{7}$ initially decomposed in the NMR sample due to the moisture introduced through addition of the substrate, complex $\mathbf{8}$ slowly formed over time. The reaction was very slow. Heating up the sample to $308 \mathrm{~K}$ was an option to accelerate the reaction and shift the reaction to the product. In the present case, the temperature was set to 298 $\mathrm{K}$ so that the reaction was slow and incomplete. It allowed to evaluate if an equilibrium was occurring between complex $\mathbf{7}$ and possible intermediates leading to complex $\mathbf{8}$. 


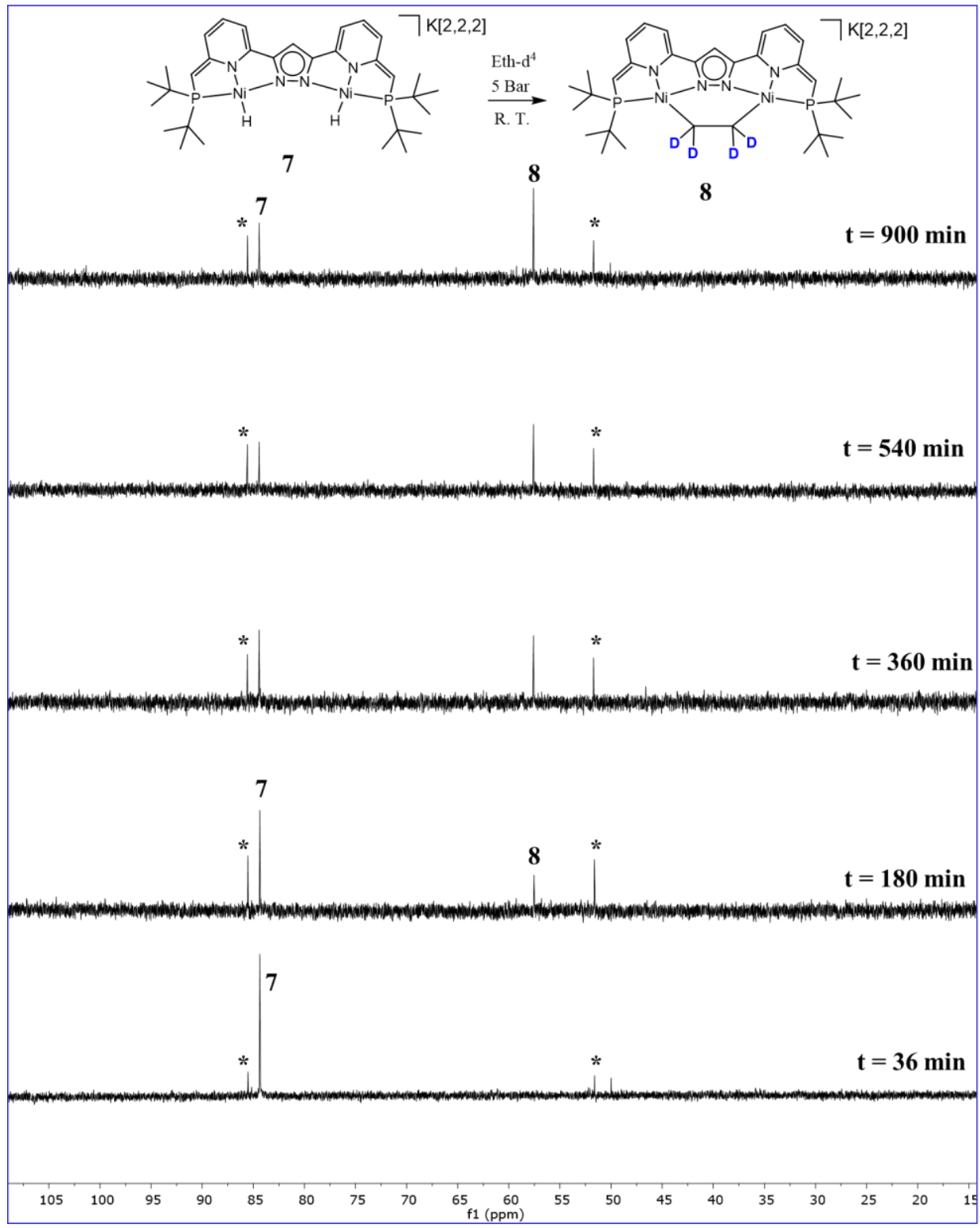

Figure 3.26. Monitoring the reaction of complex 7 to complex 8 in presence of $\mathrm{CD}_{2}=\mathrm{CD}_{2}$ by ${ }^{31} \mathrm{P}$ NMR spectroscopy in toluene-d ${ }^{8}$ at $298 \mathrm{~K}$

The monitoring of the reaction by ${ }^{1} \mathrm{H}$ NMR spectroscopy is represented in Figure 3.27. The slow character of the reaction was confirmed as not even 50\% conversion was observed after $245 \mathrm{~min}$. This conversion could also be estimated in the ${ }^{31} \mathrm{P}$ NMR spectrum in Figure 3.26 at $360 \mathrm{~min}$ where the peak of complex $\mathbf{7}$ had nearly the same intensity than complex $\mathbf{8}$. After the beginning of the reaction, small amounts of $\mathrm{H}_{2}$ were detected in solution. However, the concentration was very low as it was produced in stoichiometric amounts. The signal intensity was very low. The signal eventually disappeared after some time probably because of gas diffusion in the NMR tube. $\mathrm{A}^{2} \mathrm{H}$ NMR spectrum of this solution was recorded (Figure 3.28). Even if the solvent peaks were very large, the signal of the $\mathrm{C}_{2} \mathrm{D}_{4}{ }^{2-}$ ligand in complex $\mathbf{8}$ could be detected at $0.75 \mathrm{ppm}$ (at 400 $\mathrm{MHz}$ ). It was previously recognized in THF-d ${ }^{8}$ as a broad signal at $0.86 \mathrm{ppm}$ (at $500 \mathrm{MHz}$ ). (Figure 3.24). The difference in shift could be explained by the different solvent (THF versus THF- ${ }^{8}$ ) and the different frequencies the experiments were performed at. Thus, the signal of the $\mathrm{C}_{2} \mathrm{D}_{4}{ }^{2-}$ 
bridging unit was detected but no $\mathrm{Ni}-\mathrm{D}, \mathrm{H}-\mathrm{D}$ or $\mathrm{D}_{2}$ were detected. This observation will be used in the following discussion.

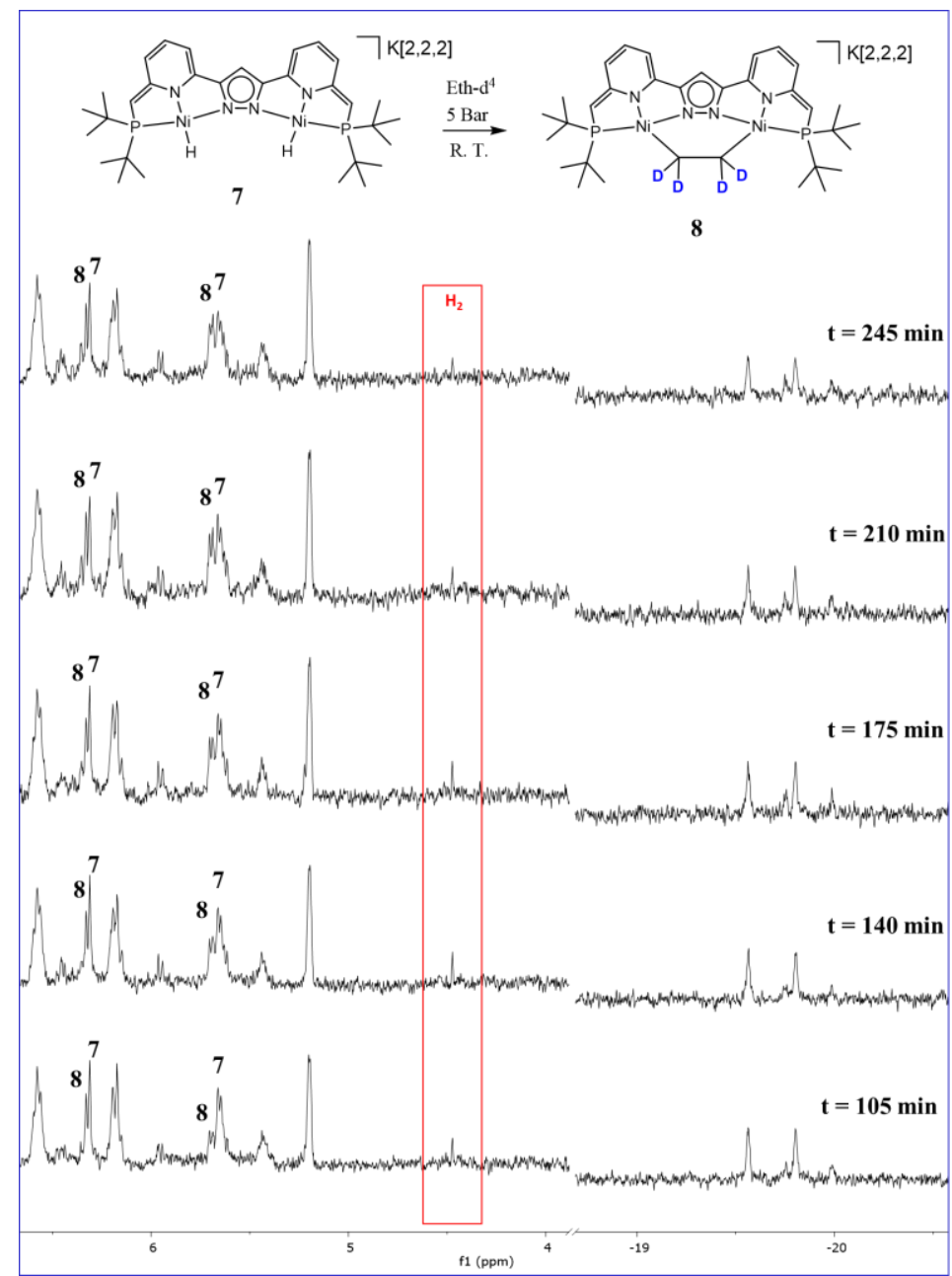

Figure 3.27. Monitoring the reaction of complex 7 to complex 8 in presence of $\mathrm{CD}_{2}=\mathrm{CD}_{2}$ by ${ }^{1} \mathrm{H}$ NMR spectroscopy in toluene- $\mathrm{d}^{8}$ at $298 \mathrm{~K}$. Spectra are truncated for more clarity.

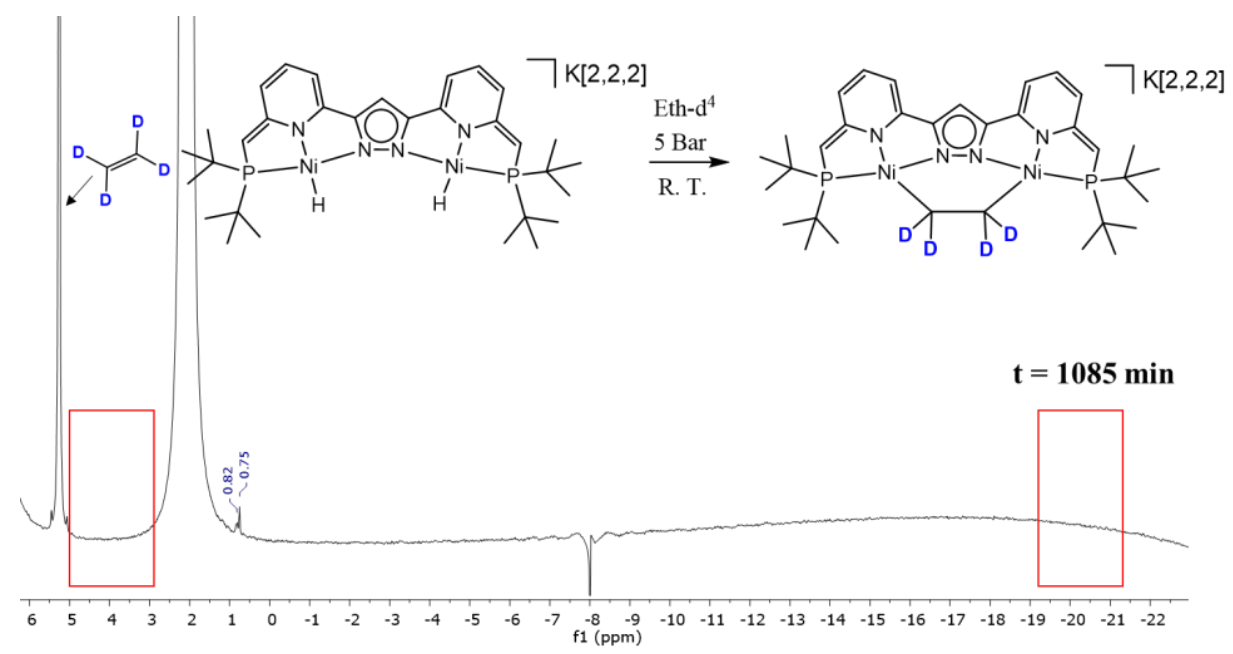

Figure 3.28. ${ }^{2} \mathrm{H}$ NMR spectrum of the reaction of complex $\mathbf{7}$ to complex $\mathbf{8}$ in presence of $\mathrm{CD}_{2}=\mathrm{CD}_{2}$ in toluene-d ${ }^{8}$ at $298 \mathrm{~K}$ after $1085 \mathrm{~min}$. 
Clear evidence of the mechanistic path of ethylene activation could not be found in the collected NMR spectra. The decrease of the ${ }^{31} \mathrm{P}$ NMR signal corresponding to complex $\mathbf{7}$ and the increase of the signal corresponding to complex $\mathbf{8}$ in Figure 3.25 should be interpreted with cautions.

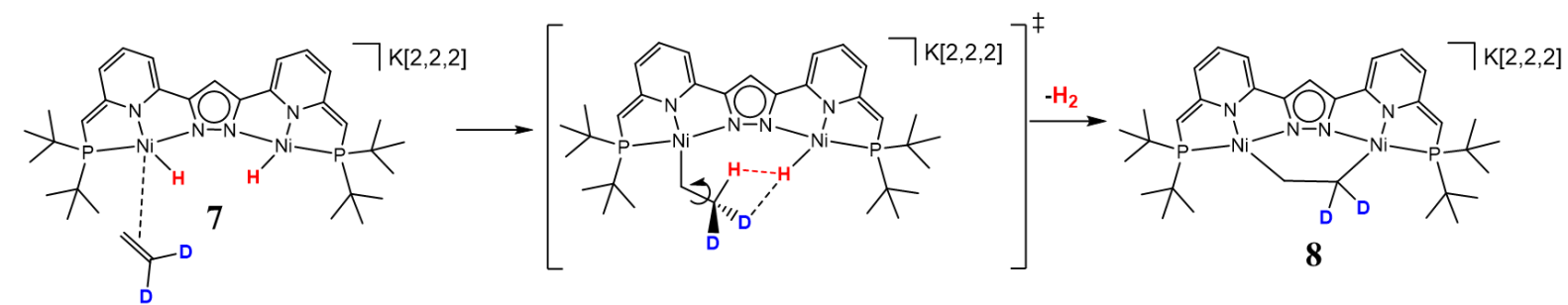

Figure 3.29. Possible mechanism for the formation of complex $\mathbf{8}$.

Olefins are known to reversibly insert in nickel-hydrogen bonds according to an insertion/ $\beta-\mathrm{H}$ elimination equilibrium. ${ }^{167,168,169}$ It is interesting to note that, if such equilibrium would take place between complex 7 and $\mathrm{CD}_{2}=\mathrm{CD}_{2}$, the $\beta$-H elimination would lead to the formation of $\mathrm{Ni}-\mathrm{D}$ moieties during the reaction. The fingerprint of such a Ni-D bond has been analysed previously. Ni-D gave typical 1:1:1 triplet on ${ }^{31} \mathrm{P}$ NMR spectroscopy (example Figure 3.15). According to spectra shown in Figure 3.25, Ni-D was not observed, and it rather excluded the possibility of $\beta$-H elimination.

The insertion of an olefin into a metal-hydrogen bond is a common reaction for $\mathrm{d}^{8}$ square planar complexes. ${ }^{170}$ Complex $\mathbf{7}$ has a $\mathrm{Ni}-\mathrm{H}$ moiety which is an interesting chemical bond regarding the oligomerization or polymerization of olefins. It is noteworthy that $\mathrm{Ni}-\mathrm{H}$ is the active core of the catalyst in the Shell Higher Olefins Process (SHOP). ${ }^{171}$ After addition of ethylene to complex 7, no oligomer was formed and heating the sample to $308 \mathrm{~K}$ was necessary to promote the reaction to complex $\mathbf{8}$. It likely indicated that the formal reduction of ethylene was kinetically hindered. A depiction of the mechanism is proposed in Figure 3.29. After the olefin insertion, a $\mathrm{CD}_{2}-\mathrm{CHD}_{2}^{-}$unit formed and the interaction with the other $\mathrm{Ni}-\mathrm{H}$ bond would led to deprotonation and formation of complex $\mathbf{8}$. This mechanism can be questioned regarding the labelling experiment in Figure 3.26. Only $\mathrm{H}_{2}$ release has been observed during the reaction. If the intermediate represented in Figure 3.31 was forming, a free rotation of the $\mathrm{C}-\mathrm{C}$ bond around the $\mathrm{CD}_{2}-\mathrm{CHD}_{2}$ unit would allow the release of $\mathrm{HD}$ during the deprotonation, but it was not the case. It is unlikely that the $\mathrm{Ni}-\mathrm{H}$ would selectively deprotonate an unactivated $\mathrm{C}-\mathrm{H}$ bond. It is however noteworthy that the interaction between a metal-hydrogen bond with a $\mathrm{C}-\mathrm{H}$ bond according to a $\mathrm{M}-\mathrm{H} \cdots \mathrm{H}-\mathrm{C}$ dihydrogen bond in the solid state has recently been analysed. ${ }^{172}$

Finally, no intermediates have been observed during the reaction of complex $\mathbf{7}$ to complex $\mathbf{8}$, which possibly indicated the occurrence of a concerted mechanism. Similar observations have been made by $\mathrm{D}$. Manz with a related dihydride dinickel complex in presence of phenylacetylene. A mechanistic proposal based on parahydrogen induced polarization (PHIP) has been formulated. ${ }^{165}$ This involved a six-center-six-membered transition state which simultaneously led to elimination of $\mathrm{H}_{2}$ and to the reduction of phenylacetylene (Figure 3.30). 


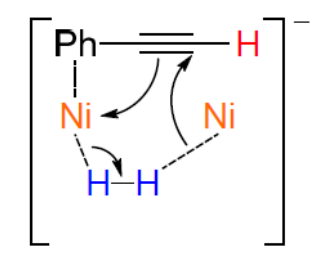

Figure 3.30. Six-center-six-membered transition state proposed by D. Manz in his dissertation. ${ }^{165}$

The combination of the two hydride ligands to give $\mathrm{H}_{2}$ in Manz's system was demonstrated by labelling experiment with NMR spectroscopy. In analogy to this mechanism, the addition of $\mathrm{CD}_{2}=\mathrm{CD}_{2}$ to $\mathrm{K}[2,2,2]\left[\mathrm{LNi}_{2}(\mathrm{H})_{2}\right]$ (complex 7) was accompanied by a release of $\mathrm{H}_{2}$ which was detected by ${ }^{1} \mathrm{H}$ NMR spectroscopy. No $\mathrm{HD}$ or $\mathrm{D}_{2}$ had been detected during reaction by ${ }^{2} \mathrm{H}$ NMR spectroscopy which likely indicated that the two hydrides of complex $\mathbf{7}$ also combined to give $\mathrm{H}_{2}$. Thus, it is possible that the same mechanism was involved in both cases. The deeper elucidation of the mechanism demanded further investigations, which were beyond the scope of this thesis.

\section{5) Conclusion:}

A new dihydride dinickel complex 7 reminiscent of the dinickel dihydride system developed by D. Manz has successfully been synthesized and characterized. It was the first doubly dearomatized Two-In-One pincer complex that was structurally characterized. The doubly dearomatized state represents a unique and attractive platform to study metal-metal and metal-ligand cooperativity. The reaction of complex 7 with $\mathrm{H}_{2}$ led to the conclusion that the side arm was not involved in the activation of hydrogen. The exchange was taking place sequentially on each nickel atoms, as the labelling experiment revealed the exclusive formation of HD. The mechanism of $H / D$ exchange was different than the one involved in the system developed by D. Manz, who observed the exclusive formation of $\mathrm{D}_{2}$. Complex 7 was then tested in presence of ethylene. No polymerization or oligomerization were observed. Complex 8 eventually formed and involved a $\mathrm{C}_{2} \mathrm{H}_{4}{ }^{2-}$ bridging unit. Possible mechanisms were discussed based on the available experimental data, but its elucidation requires further investigations. Eventually, all reactions performed on complex $\mathbf{7}$ in this chapter likely indicated the absence of cooperative effects by (de)aromatization of the ligand backbone. In the next chapter, new reactions implicating the ligand cooperation will be discussed. 


\section{Chapter 4: Ligand cooperation and sequestration of an intramolecular dihydrogen bond.}

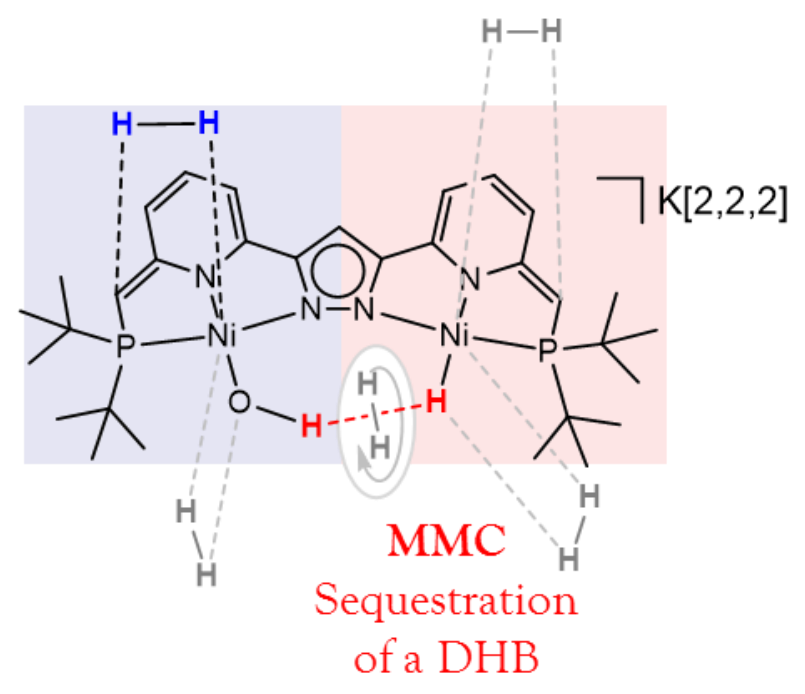

\subsection{The dihydrogen bond (DHB) in organometallic chemistry.}

Different types of hydrogen bonds were classified in the course of the 20th century. ${ }^{173}$ Classical hydrogen bonds were understood as the interaction between a $\mathrm{X}-\mathrm{H}$ weakly acidic polar proton donor entity ( $\mathrm{X}$ being a heteroatom such as $\mathrm{O}$ or $\mathrm{N}$ ) and the lone pair (l.p.) of a proton acceptor. The resulting interacting fragment was represented as follows: $\{\mathrm{X}-\mathrm{H} \cdots 1 . \mathrm{p}\} .{ }^{174}$ Non-classical hydrogen bonds were interactions featuring a new type of proton acceptor. Indeed, $\pi$ aromatic systems such as benzene or acetylene had a basic character and were also able to interact with a proton according to a $\{\mathrm{X}-\mathrm{H} \cdots \pi\}$ motif. ${ }^{173}$

\section{Hydrogen, the most abundant element in the universe, always " seems to find new roles to play in the chemical theater. " 175}

The enthusiasm rose with the discovery of a curious hydrogen bond involving two differently polarized hydrogen atoms according to a $\{\mathrm{X}-\mathrm{H} \cdots \sigma\}$ motif ( $\sigma$ representing the $\sigma$ bond between a different $\mathrm{H}-\mathrm{Y}$ fragment. $\mathrm{Y}$ could be $\mathrm{C}, \mathrm{B}$ or $\mathrm{M}$ (a metal)). This interaction was firstly acknowledged in 1960s in boron coordination compounds (for example: $\mathrm{Et}_{3} \mathrm{~N}-\mathrm{BH}_{3}$ ) and its implication on physical properties was studied. ${ }^{176}$ Since it implied two hydrogen atoms, this hydrogen bond was called dihydrogen bond (DHB). This unconventional dihydrogen bond was reviewed in 1996. ${ }^{177}$ The review was later extended to all existing DHB compounds in 2001. ${ }^{178}$ Energetic parameters were discussed. ${ }^{179,180}$ In 2016, a review focused on DHB implicated in transition metal complexes. ${ }^{181}$ 
One of the first transition metal complexes with a recognized DHB was synthesized in 1986 by oxidative addition of $\mathrm{H}_{2} \mathrm{O}$ on $\left[\mathrm{Ir}(\mathrm{I}) \mathrm{PMe}_{4}\right] \mathrm{PF}_{6}$ (Figure 4.1). ${ }^{182}$ The intramolecular $\mathrm{H}^{\delta-} \ldots \mathrm{H}^{\delta+}$ interaction was later studied by means of neutron scattering where an interatomic distance of $2.40(1) \AA$ between the two interacting protons was found. ${ }^{183}$ Since two interacting protons are supposed to have a distance smaller than or equal to the sum of their Van der Waals radii (precisely $2.4 \AA$ ), the DHB was ambiguously demonstrated. However, the observation of a $\mathrm{Ir}-\mathrm{O}-\mathrm{H}$ bent angle toward the $\mathrm{Ir}-\mathrm{H}$ bond strongly suggested the existence of attractive interaction of the two hydrogens. Similar $(\mathrm{H}) /(\mathrm{OH})$ hydrido/hydroxyl osmium based complexes were synthesized but the DHB was not discussed. ${ }^{184}$ Meanwhile, the DHB was related to an attractive "cis-effect" between the hydride and the $\mathrm{H}_{2}$ ligands in a $\mathrm{Fe}(\mathrm{H})_{2}\left(\mathrm{H}_{2}\right)\left(\mathrm{PEtPh}_{2}\right)_{3}$ complex. ${ }^{185}$ Four-membered DHB rings were later confirmed in Os and Ru complexes. ${ }^{186}$

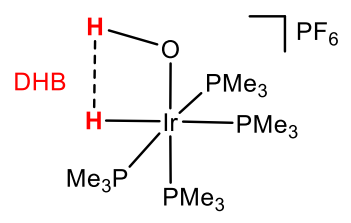

\section{6}

Figure 4.1. First DHB complex involving a transition metal. ${ }^{182}$

The occurrence of DHB in a chemical reaction was proposed: in 1994, another iridium complex involved an intramolecular DHB with noteworthy properties regarding dihydrogen activation (Figure 4.2). ${ }^{187}$ The terminal hydride bound to Ir was interacting with the proton of the pendant pyridinium ligand (Figure 4.2). The two interacting hydrogen atoms reversibly formed a covalent bond and the resulting $\mathrm{H}_{2}$ released could be exchanged with $\mathrm{D}_{2}$. The interaction was deactivated by changing the apolar $\mathrm{CH}_{2} \mathrm{Cl}_{2}$ solvent with THF, whereby the activity toward $\mathrm{D}_{2}$ was not observed. The NMR characteristics of the DHB disappeared. The intramolecular DHB present in the iridium complex supposedly lowered the energy of the transition state during the proton transfer reaction. A case of intermolecular DHB was later demonstrated in a Ru complex. ${ }^{188}$

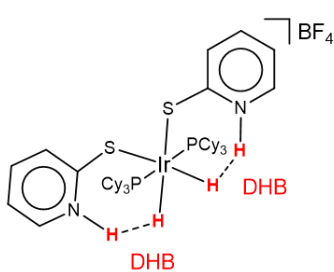

In $\mathrm{CH}_{2} \mathrm{Cl}_{2}$ $\mathrm{ON}$

Splitting $\mathrm{H}_{2}$

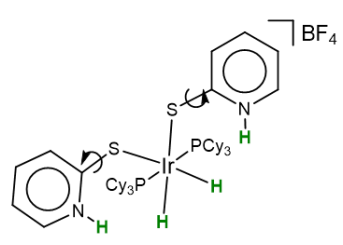

In THF

$\mathrm{OFF}$

Not Splitting $\mathrm{H}_{2}$

Figure 4.2. Switching OFF/ON a dihydrogen bond depending on the solvent. ${ }^{187}$

The implication of DHB in a catalytic reaction was highlighted in a $\mathrm{Ru}$ complex with the hydrogenation of $\mathrm{CO}_{2}$ into formic acid. ${ }^{189} \mathrm{~A}$ similar DHB was involved in another Ru complex that catalytically reduced nitriles into amides (Figure 4.3). ${ }^{190}$ 


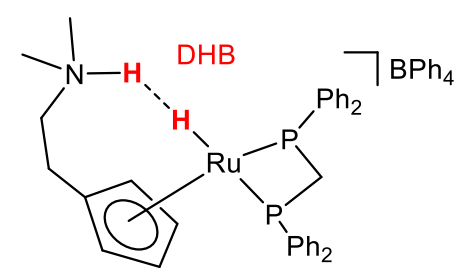

1998

Cat

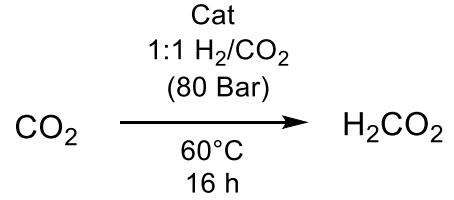

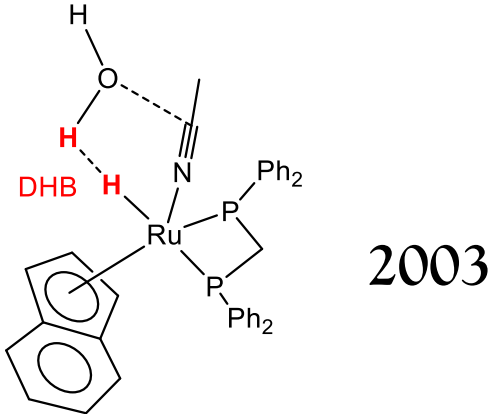

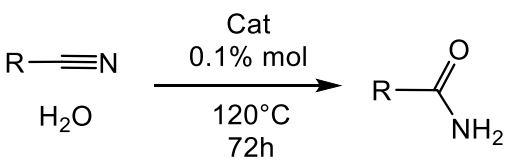

Figure 4.3. Catalytic hydrogenation of $\mathrm{CO}_{2}$ to formic acid ${ }^{189}$ and hydration of nitriles to amides promoted by Ru complexes that were proposed to feature a DHB.

Critical reconsideration of Noyori's hydrogenation reaction was recently performed by means of DFT. ${ }^{191}$ The formation of the $\eta^{2}-\mathrm{H}_{2}$ ligand was discussed. The combination of the hydride with the hydrogen of the $\mathrm{N}-\mathrm{H}$ ligand to give $\mathrm{H}_{2}$ was unlikely as the energy profile of this reaction was too high. Instead, the formation of the $\eta^{2}-\mathrm{H}_{2} \mathrm{Ru}$ intermediate was proposed to occur through the $\{\mathrm{RO}-\mathrm{H} \cdots \mathrm{H}-\mathrm{Ru}\} \mathrm{DHB}$ adduct formed between the alcohol $(\mathrm{RO}-\mathrm{H})$ and the $\mathrm{Ru}-\mathrm{H}$ moiety of the complex. The activation barrier was lowered by c.a. $14.9 \mathrm{kcal}^{\mathrm{mol}}{ }^{-1}$ (Figure 4.4).

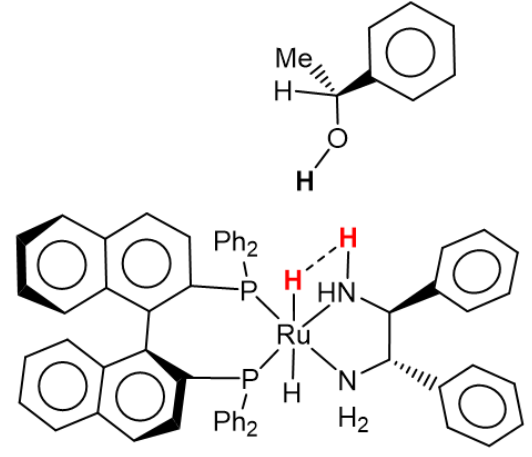

27.7 kcal.mol ${ }^{-1}$

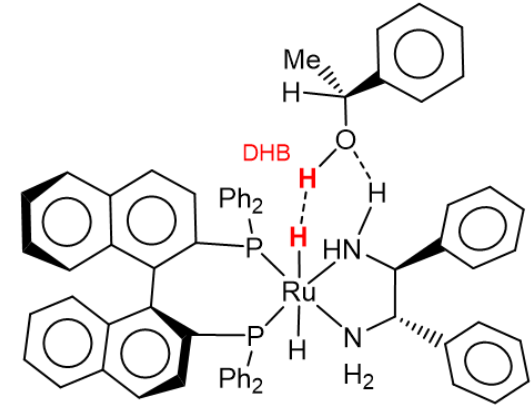

12.8 kcal.mol ${ }^{-1}$

Figure 4.4. New calculcated transition state during Noyori's hydrogenation. ${ }^{191}$

The implication of hydride or dihydrogen complexes in electrocatalytic hydrogenation processes have been analysed. ${ }^{192}$ Electrochemistry was a valuable method to study the mechanism for the formation of dihydrogen. ${ }^{193}$ There was a variety of electrocatalysts that could be exploited for such objectives. A series of such electrocatalysts is represented in Figure 4.5. Those complexes had a pendant amine arm in the second coordination sphere of the metal. It played the role of proton acceptors or "proton relay" (examples $\mathbf{C},{ }^{194} \mathbf{A},{ }^{143} \mathbf{B},{ }^{195} \mathbf{D},{ }^{196} \mathbf{E}^{197}$ ). Complex B was crystallized and the DHB was evidenced. ${ }^{195}$ The DHB could not be evidenced in complexes C, D and $\mathbf{E}$ however, the two hydrogen atoms represented in red (Figure 4.5) were exchanged even at low temperature 
and short relaxation times in case of $\mathbf{E}$ for the hydride NMR signal suggested an attractive interaction between the hydride and the proton.

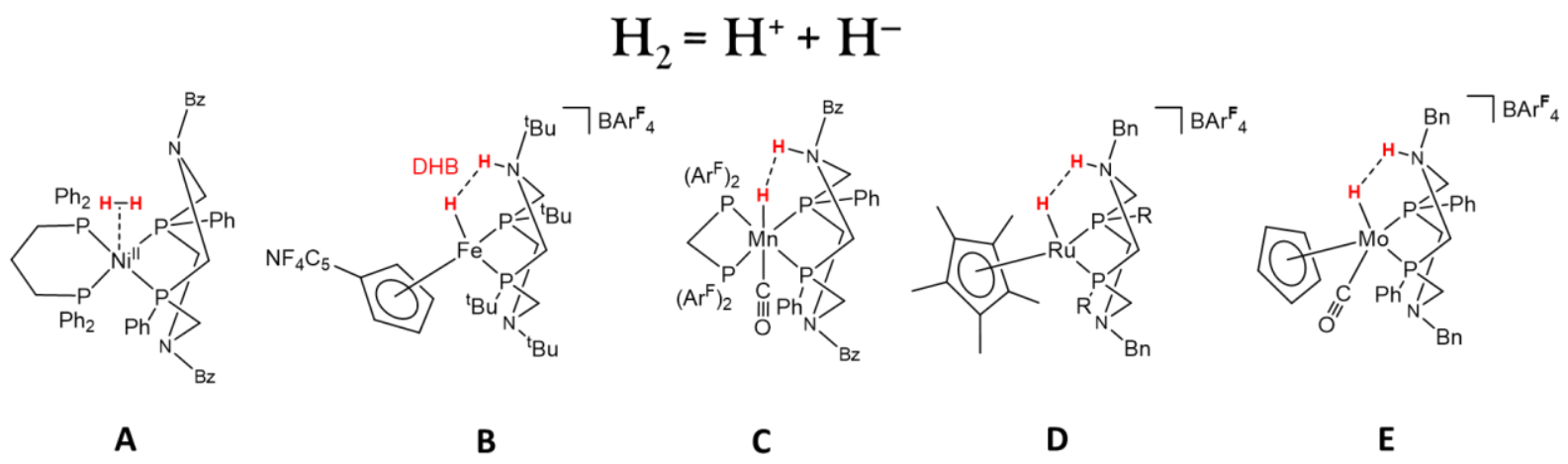

Figure 4.5. Electrocatalysts that can reversibly activate $\mathrm{H}_{2}$ through the formation of a DHB. Examples of complex $\mathbf{A},{ }^{129} \mathbf{B},{ }^{195} \mathbf{C},{ }^{194} \mathbf{D}^{196}$ and $\mathbf{E}^{197}$ are shown.

Hydrogen bond networks induced by the presence of water sometimes played an important role to overcome the energetic barrier of $\mathrm{H}_{2}$ activation. ${ }^{198}$ Adding water in a system often rationalized the feasibility of such reaction. However, it could lead to more sophisticated mechanisms. In a dihydride $\mathrm{Ru}-\mathrm{H}$ complex represented in Figure 4.6, the $\mathrm{N}-\mathrm{H}$ moiety of the ligand was responsible for a stereoselective proton/hydride exchange occurring in presence of water. Exchange rates between the $\mathrm{Ru}-\mathrm{H}$ and water were calculated. It was demonstrated that $\mathrm{H}_{2} \mathrm{O}$ exchanged faster with the hydride close from the $\mathrm{N}-\mathrm{H}$ of the ligand. ${ }^{199}$ Removing the hydrogen atom of the nitrogen by methylation inhibited this exchange. In conclusion, the occurrence of a hydrogen bond in solution was demonstrated to promote an exchange path.

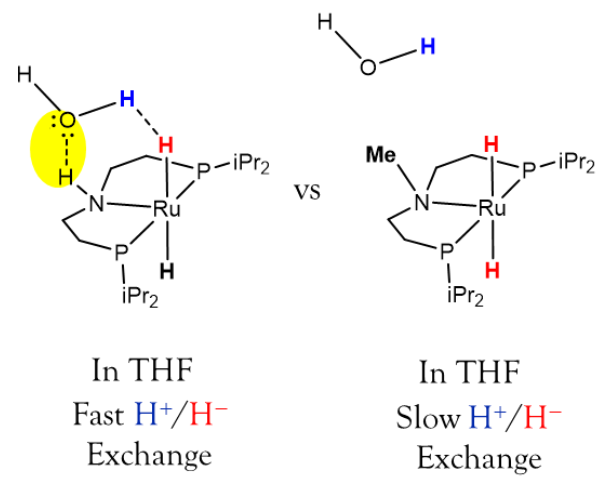

Figure 4.6. $\mathrm{H}^{+} / \mathrm{H}^{-}$exchange promoted by the presence of water in a dihydride $\mathrm{Ru}-\mathrm{H}$ complex. ${ }^{199}$

The distinction between a hydrogen and a dihydrogen bond is a challenging task as hydrogen bond mostly prevailed in solution over the other one. The interaction between $\mathrm{H}^{+} / \mathrm{H}^{-}$possibly evolves to the formation of dihydrogen complexes. Such adducts have been reviewed for 
transition metals. ${ }^{200,201}$ In this regard, the fluxionality between $\mathrm{M}\left(\eta^{2}-\mathrm{H}_{2}\right)$ and $\mathrm{M}(\mathrm{H})_{2}$ in polyhydride systems was considered. 202,203

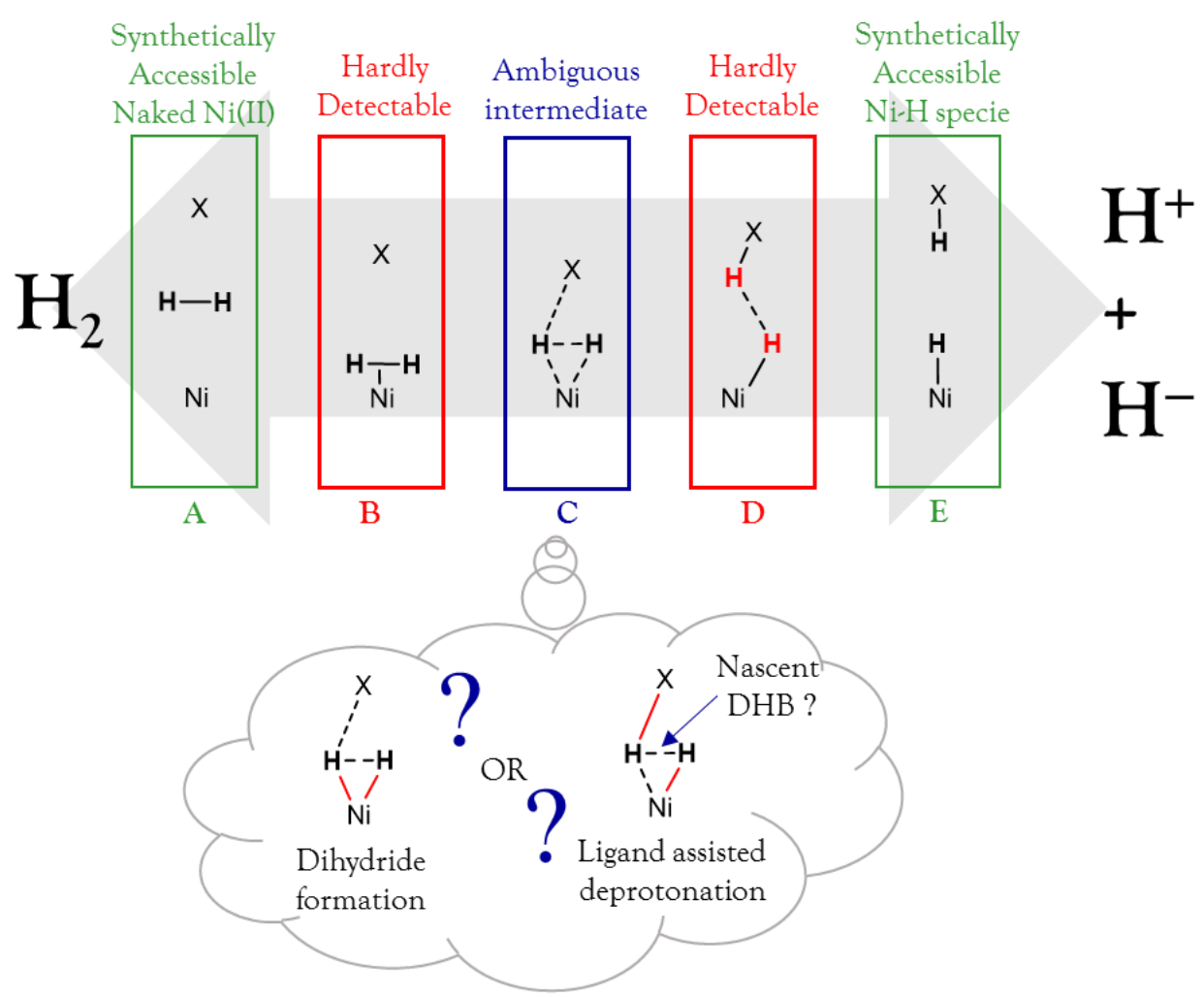

Figure 4.7. Representation of a problematic concerning the ambiguous intermediate in the blue box. For nickel(II) complexes, experimental evidences for the mechanistic path of $\mathrm{H}_{2}$ activation were difficult to obtain. ${ }^{143,147,208}$

The mechanism concerning the heterolytic splitting of $\mathrm{H}_{2}$ into $\mathrm{H}^{+} / \mathrm{H}^{-}$by $\mathrm{Ni}^{\mathrm{II}}$ complexes was proposed as shown in Figure 4.7. Addition of $\mathrm{H}_{2}$ to $\mathrm{Ni}^{\mathrm{II}}$ complexes was represented in the green box $\mathrm{A}$. If $\mathrm{H}_{2}$ coordinated to the complex, it gave rise to a $\mathrm{Ni}^{\mathrm{II}}\left(\eta^{2}-\mathrm{H}_{2}\right)$ adduct (Figure 4.7, square B). Such complexes were unstable and were hard to detect. ${ }^{204,205,206}$ The characterization of those complexes represented a possible NMR benchmark for the detection of intermediates during catalytic events. The formation of such adduct possibly evolved to an ambiguous intermediate $\mathrm{C}$ represented in the blue box. The possibility that $\mathrm{Ni}^{\mathrm{II}}\left(\eta^{2}-\mathrm{H}_{2}\right)$ oxidized into $\mathrm{Ni}^{\mathrm{IV}}\left(\mathrm{H}_{2}\right)$ has been proposed. ${ }^{147,143}$ However, the oxidative addition of $\mathrm{H}_{2}$ to give dihydride complexes was commonly more accepted for $\mathrm{Ni}^{0}$ complexes. ${ }^{151}$ An alternative pathway was to consider the polarization of the $\mathrm{H}-\mathrm{H}$ bond prior to bond breaking. The coordination of $\mathrm{H}_{2}$ on the Lewis acidic metal and surrounding polar ligands (for example the proton relay in Figure 4.5) facilitated the polarizability of $\mathrm{H}-\mathrm{H}$ into ${ }^{\delta+} \mathrm{H}-\mathrm{H}^{\delta-}$. The breaking of $\mathrm{H}-\mathrm{H}$ gave the two fragments represented in the box $\mathrm{E}$ (Figure 4.7) and it was possibly mediated by a ${ }^{\delta+} \mathrm{H} \cdots \cdot \mathrm{H}^{\delta-}$ dihydrogen bond (Figure 4.7, square D). 
An interesting case is summarized in Figure 4.8. The synthesis and the characterization of a $\mathrm{Ni}^{\mathrm{II}}-\mathrm{H}$ complex $(\mathbf{F})$ was established ${ }^{207}$ and the addition at low temperature of an acid to this complex led to the generation of a DHB intermediate $(\mathbf{G}){ }^{208}$ The adduct decomposed at room temperature. A Ni- $\left(\eta^{2}-\mathrm{H}_{2}\right)$ motif was characterized $(\mathbf{H})$ in this case. ${ }^{204}$ The minimum of ${ }^{1} \mathrm{H}$ NMR relaxation time for the $\mathrm{Ni}-\mathrm{H}$ signal was measured in each step of the transformation (Figure 4.8). The distance between the two red hydrogen atoms was estimated based on NMR spectroscopy in the case of complexes $\mathbf{G}$ and $\mathbf{H}$.

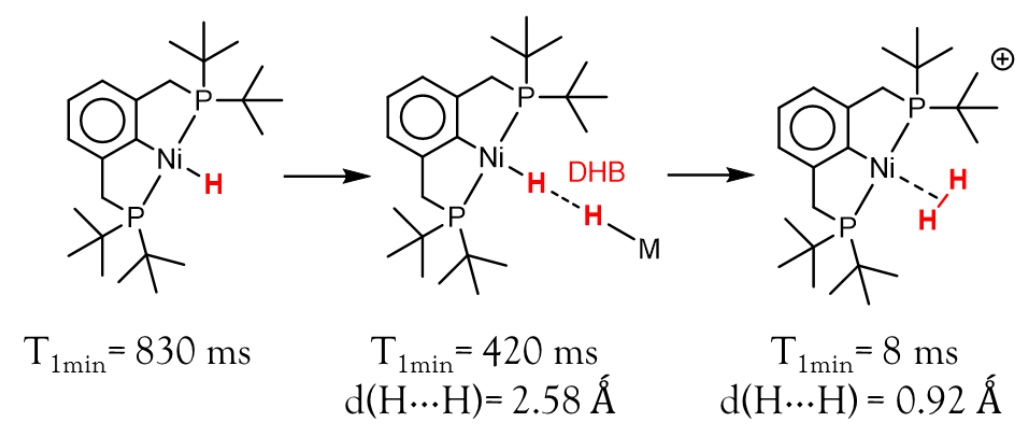

Figure 4.8. Protonation of a Ni-H hydride mediated by a DHB and evolution of the $\mathrm{T}_{1} \mathrm{NMR}$ relaxation time. ${ }^{204,207,208}$

An interesting case of dialuminium complex is represented in Figure 4.9. It featured a transition state possibly mediated by an intramolecular DHB. ${ }^{209}$ The DHB was found to play a critical role in the stabilization of intermediates during methanolysis.<smiles></smiles>

2001

Figure 4.9. Hypothetical transition state proposed in a pyrazolate based dialuminium complex. ${ }^{209}$

An attentive lecture of Manz's dissertation (Part 4.5, "reaction with water") ${ }^{165}$ revealed appealing details. It was assumed that the addition of one equivalent of water to the dihydride dinickel complex represented in Figure 4.10 gave rise to an asymmetric hydrido/hydroxo complex $\mathbf{I}$. The NMR features of this proposed complex I were in line with the formation of an intramolecular DHB adduct. However, this asymmetric intermediate was unstable and decomposed overtime to give an inert hydroxo complex $\mathbf{J}$. The isolation of complex $\mathbf{I}$ and the full characterization of the hypothetical DHB was a challenging task as it was unstable. In this chapter, a similar hydrido/hydroxo pyrazolate based dinickel complex was isolated and fully characterized. Its features will be presented and discussed. 


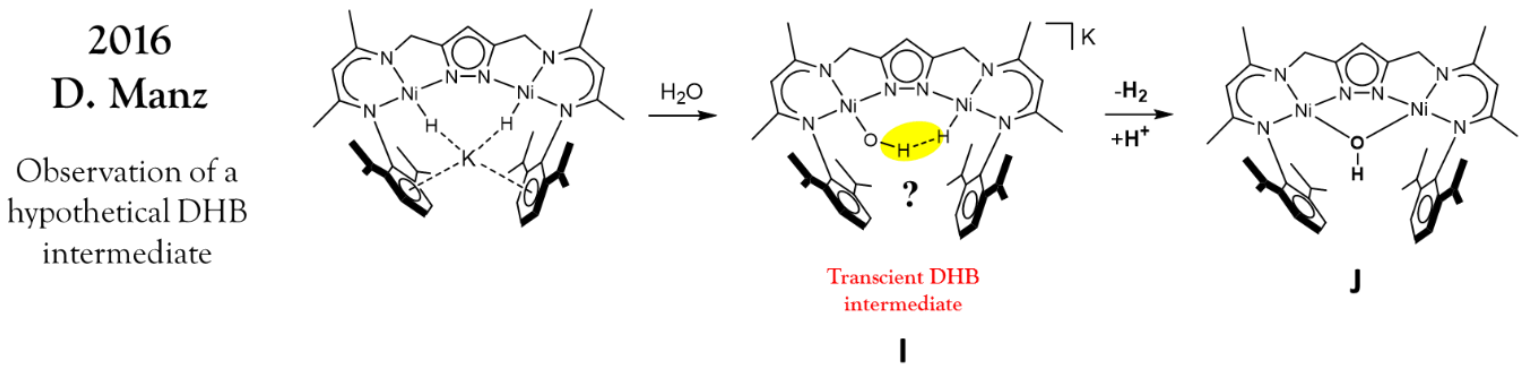

Figure 4.10. Reaction of a hydrido hydroxo complex $\mathbf{I}$, decomposing to a stable complex $\mathbf{J}$ discussed by Manz. ${ }^{165}$

Nuclear magnetic resonance (NMR) is well suited to investigate DHB adducts. The spectroscopic fingerprints of this interaction have been listed in Figure 4.11. Even if NMR spectroscopic data give strong evidence for the occurrence of a DHB, it must be supported by calculations and other analytic methods like IR spectroscopy and neutron diffraction if possible.

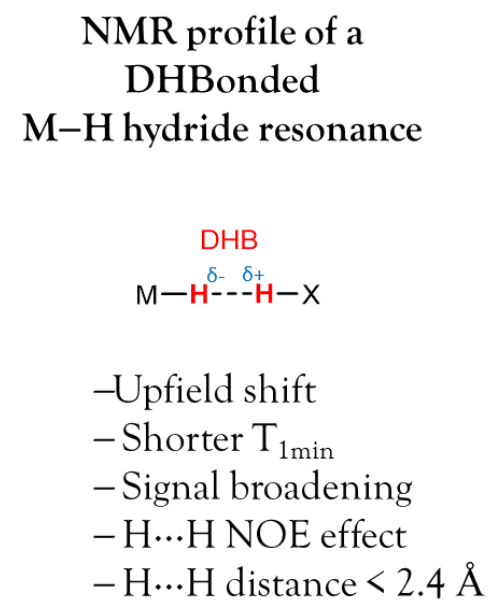

Figure 4.11. NMR features of $\mathrm{M}-\mathrm{H}$ involved in a DHB. ${ }^{181}$

\subsection{Isolation of a stable hydrido hydroxo dinickel complex.}

The moisture sensitivity of complex $\mathbf{7}$ was previously suggested by the appearance of additional NMR signals during the study of ethylene activation monitored by ${ }^{31} \mathrm{P}$ NMR spectroscopy. To identify the complex formed, a sequential addition of $\mathrm{H}_{2} \mathrm{O}$ to complex $\mathbf{7}$ was monitored by ${ }^{1} \mathrm{H}$ NMR spectroscopy (Figure. 4.12). After the addition of one equivalent of $\mathrm{H}_{2} \mathrm{O}$, the subsistence of a upfield shifted hydride resonance was observed at $-20.5 \mathrm{ppm}$. A new ${ }^{1} \mathrm{H}$ NMR peak at -1.8 ppm and three other peaks in the region of aromatic signals were observed. Those peaks were equally integrating for 1 . Only one signal for a pyrazolate $\mathrm{C}-\mathrm{H}$ was observed, which suggested the presence of a single complex in solution. A full ${ }^{1} \mathrm{H}$ NMR spectrum is represented in Figure 4.14. Two signals were observed by ${ }^{31} \mathrm{P}$ NMR spectroscopy (Figure 4.13). $\mathrm{A}^{31} \mathrm{P}$ NMR signal at $84.9 \mathrm{ppm}$ was close from the ${ }^{31} \mathrm{P}$ NMR signal of complex 7 (84.1 ppm). Another signal at 50.3 ppm was observed. The noticeable equal intensities of the ${ }^{31} \mathrm{P}$ NMR signals were consistent with the fact that the two ${ }^{31} \mathrm{P}$ NMR signals would belong to the same complex. 

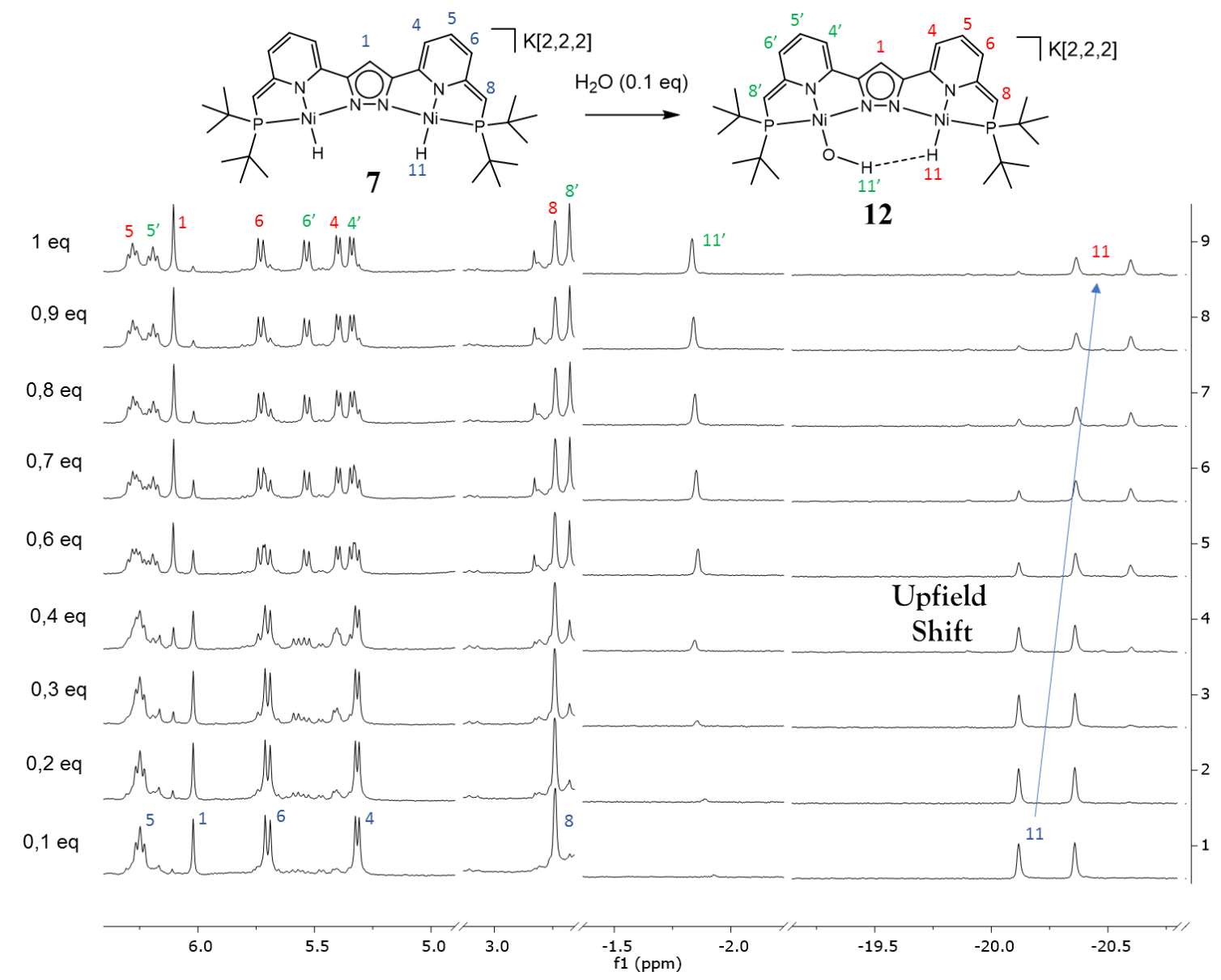

Figure 4.12. Monitoring the titration of complex 7 with $\mathrm{H}_{2} \mathrm{O}$ by ${ }^{1} \mathrm{H}$ NMR spectroscopy.
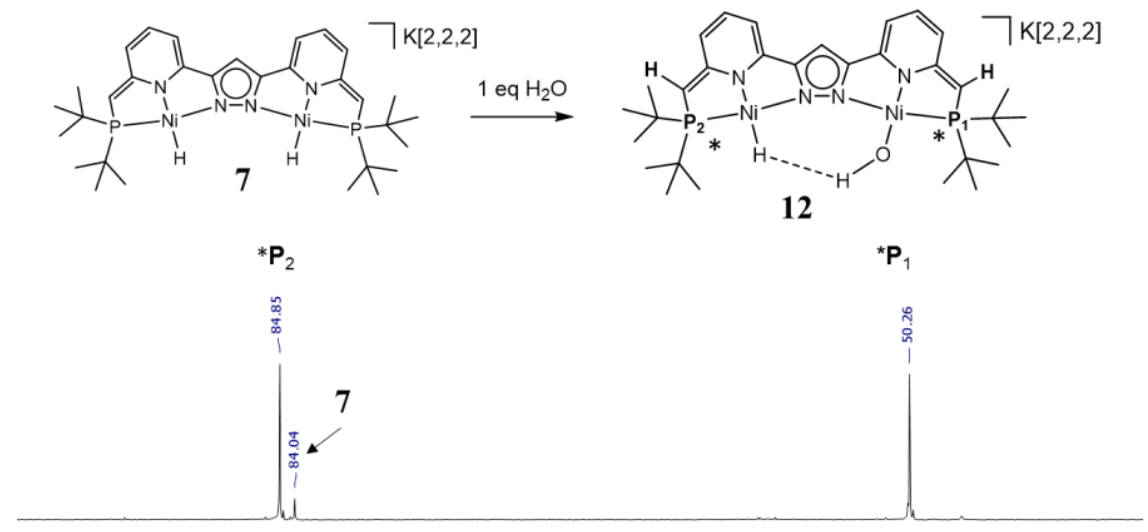

$\begin{array}{lllllllllllllllllllllllllllllllllll}98 & 96 & 94 & 92 & 90 & 88 & 86 & 84 & 82 & 80 & 78 & 76 & 74 & 72 & 70 & 68 & 66 & 64 & 62 & 60 & 58 & 56 & 54 & 52 & 50 & 48 & 46 & 44 & 42 & 40 & 3 \varepsilon\end{array}$

Figure 4.13. ${ }^{31} \mathrm{P}$ NMR spectrum of complex 7 after addition of 1 equivalent of water inTHF$\mathrm{d}^{8}(202 \mathrm{MHz})$. 


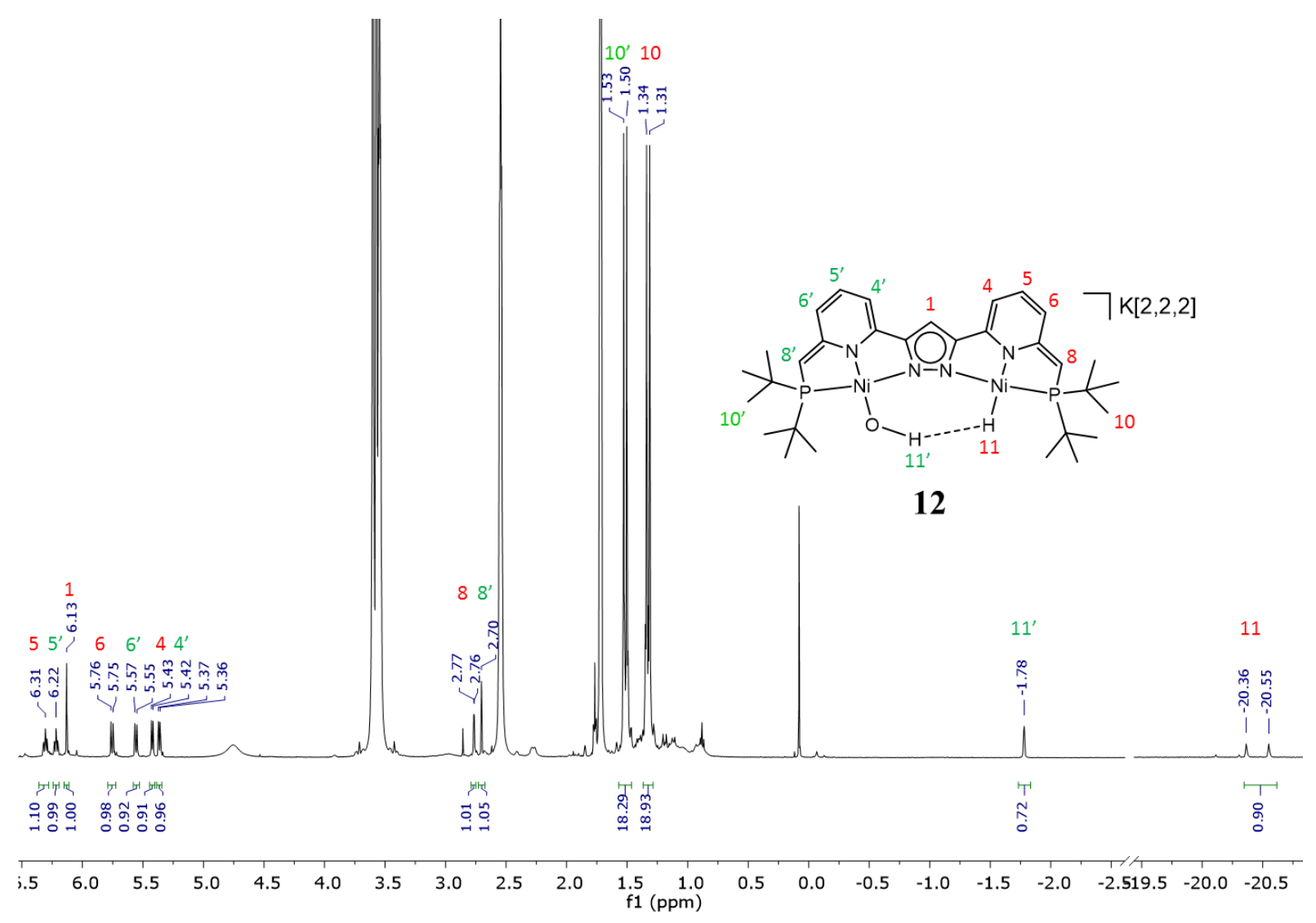

Figure 4.14. Complete ${ }^{1} \mathrm{H}$ NMR spectrum of complex 12 inTHF-d ${ }^{8}(500 \mathrm{MHz})$.

Finally, the complex was crystalized and gave the structure represented in Figure 4.15. The hydroxo and hydride ligands are disordered within the bimetallic cleft of complex $\mathbf{1 2}$ and the interpretation of bond lengths was limited. A precise location of the hydrogen atoms was subject to uncertainties in the data. However, the presence of a stoichiometric amount of oxygen in the complex could be confirmed. It supported the existence of a hydroxyl ligand binding to a $\mathrm{Ni}^{2+}$ ion. An analysis of the ligand backbone could evidence that the two pyridines were dearomatized (similar analysis as in Figure 3.21).

An ESI-MS spectrum originally measured for the characterization of complex 7 was represented in Figure 4.16. Isotopic peak distributions have been simulated and compared with the experimental results. A peak distribution with two major peaks at $\mathrm{m} / \mathrm{z}=653$ and $\mathrm{m} / \mathrm{z}=655$ corresponded to complex 7. Another isotopic distribution with two major peaks at $\mathrm{m} / \mathrm{z}=669$ and $\mathrm{m} / \mathrm{z}=671$ were observed and corresponded to complex 12. Thus, the presence of both complex 7 and complex 12 were observed during the same measurement. The sensitivity of complex $\mathbf{7}$ toward water probably led to the formation of complex $\mathbf{1 2}$ during the measurement. 


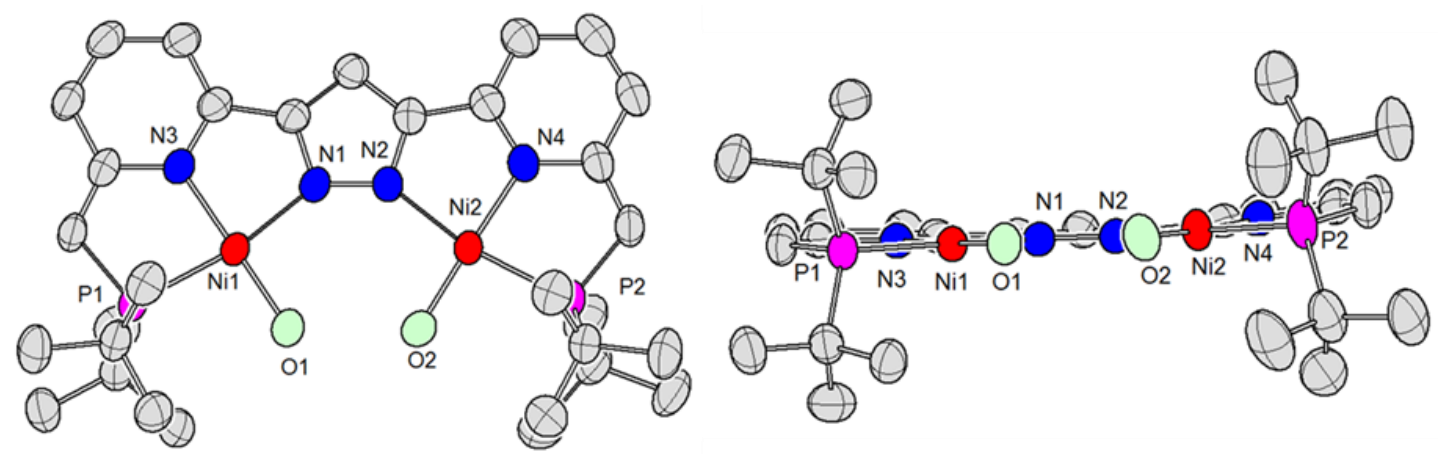

Figure 4.15 Molecular structure (thermal displacement ellipsoids shown at $50 \%$ probability) of the anion of complex 12. Hydrogen atoms and all cations have been omitted for clarity. The hydride atom could not be localized because of disorder of the $\mathrm{HO}^{-}$moiety. Left: top view of the molecular structure. Right: front view of the molecular structure

\begin{tabular}{cc|cc}
\hline \multicolumn{2}{l|}{ Bond Lengths around $\mathrm{Ni}(1) / \AA$} & \multicolumn{2}{l}{ Bond Lengths around $\mathrm{Ni}(2) / \AA$} \\
\hline & & & \\
\hline $\mathrm{Ni}(1)-\mathrm{O}(1)$ & $1.810(3)$ & $\mathrm{Ni}(2)-\mathrm{O}(2)$ & $1.802(5)$ \\
\hline $\mathrm{Ni}(1)-\mathrm{N}(3)$ & $1.896(2)$ & $\mathrm{Ni}(2)-\mathrm{N}(4)$ & $1.903(2)$ \\
\hline $\mathrm{Ni}(1)-\mathrm{N}(1)$ & $1.940(2)$ & $\mathrm{Ni}(2)-\mathrm{N}(2)$ & $1.953(2)$ \\
\hline $\mathrm{Ni}(1)-\mathrm{P}(1)$ & $2.1635(8)$ & $\mathrm{Ni}(2)-\mathrm{P}(2)$ & $2.1564(8)$ \\
\hline
\end{tabular}

Table 4.1 Selected bond lengths

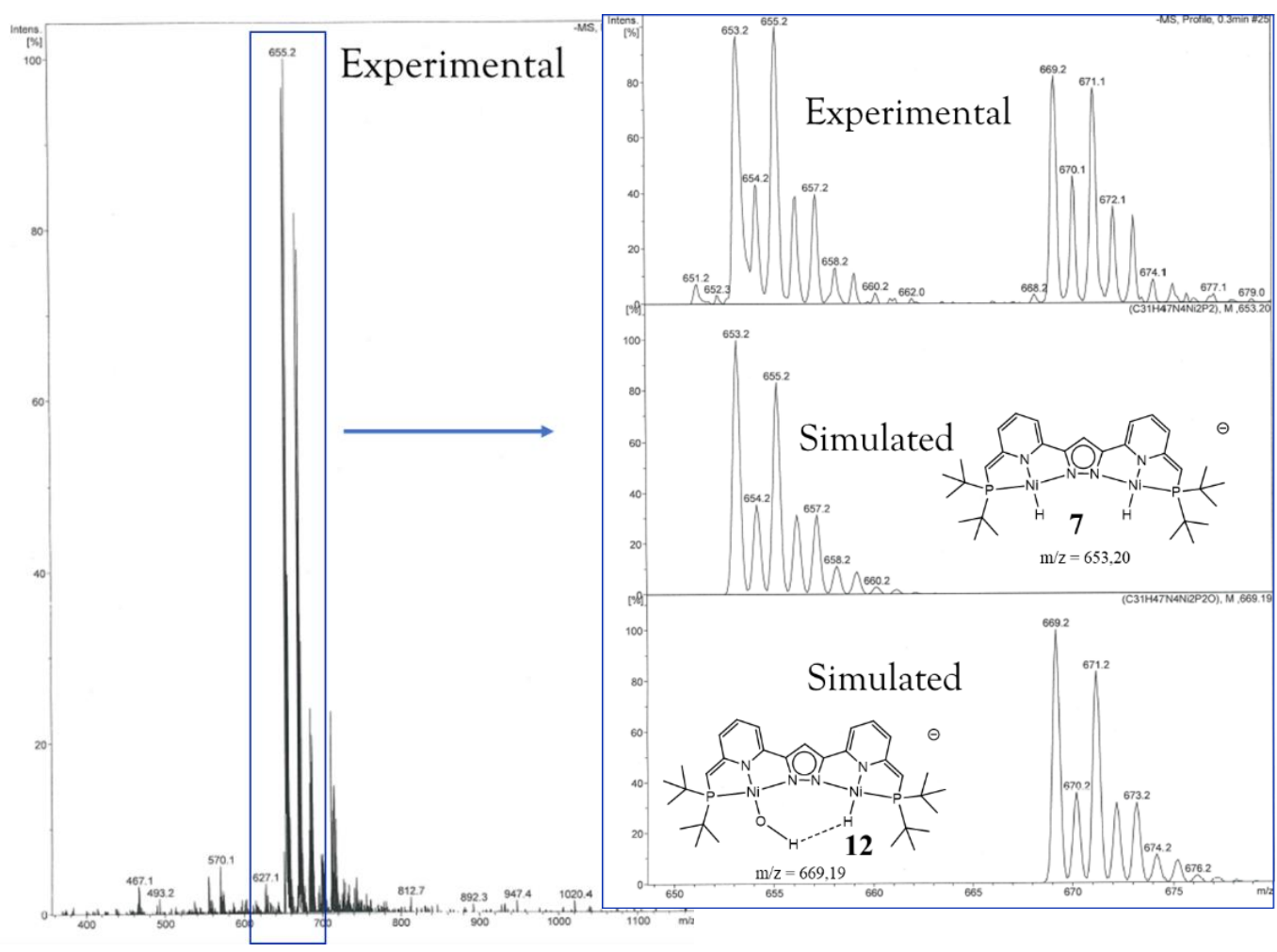

Figure 4.16. ESI-MS spectrum of complex 7 and complex 12 in THF. The inset shows the experimental and simulated isotropic distribution pattern for $\mathrm{LH}_{2} \mathrm{Ni}_{2}(\mathrm{H})_{2}{ }^{-}$and for $\mathrm{LH}_{2} \mathrm{Ni}_{2}(\mathrm{OH})(\mathrm{H})^{-}$. 


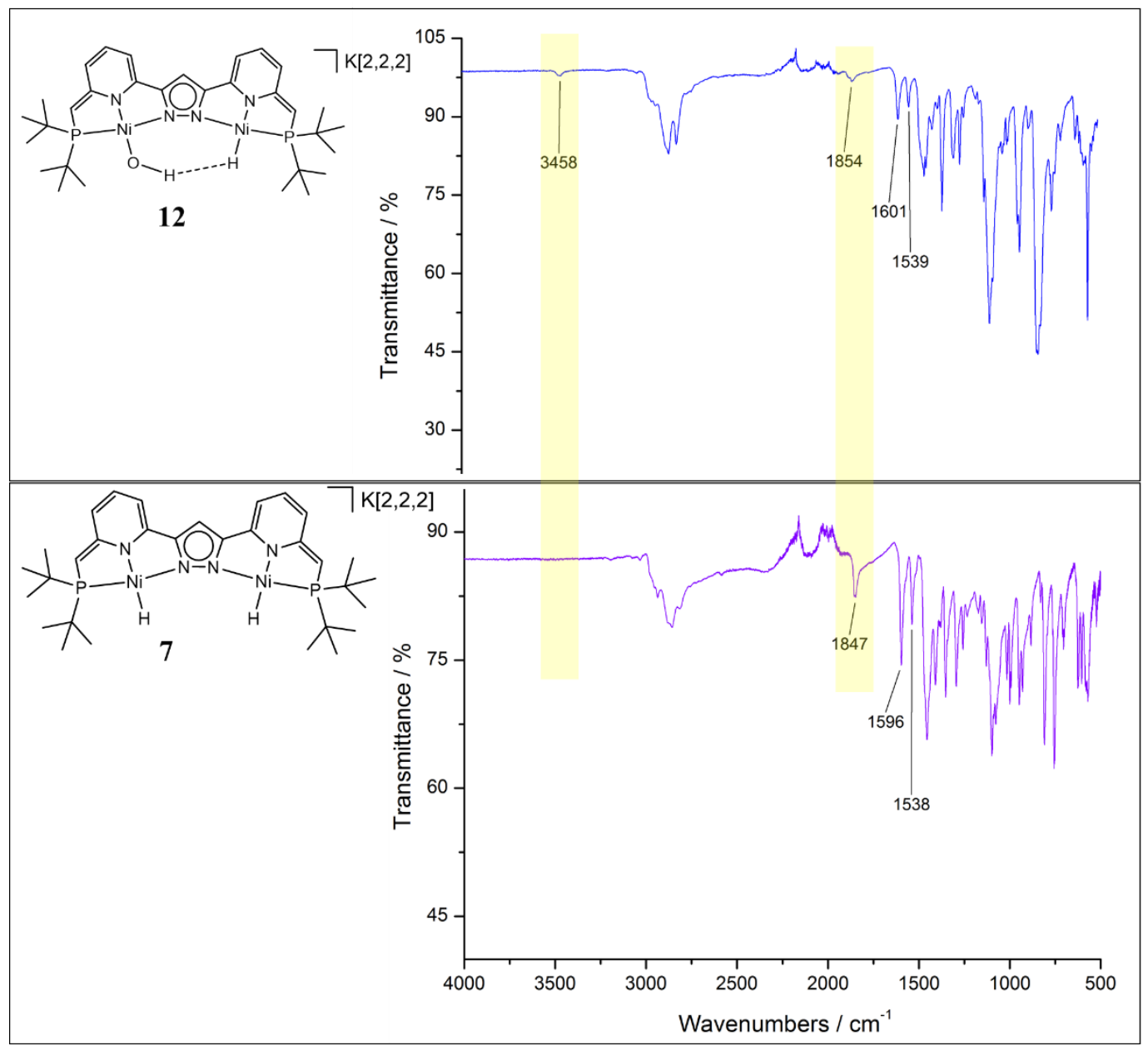

Figure 4.17. Comparison of the ATR-IR spectra in the solid state of complex 7 (bottom) and complex 12 (top).

An ATR-IR spectrum of complex 12 in the solid is represented in Figure 4.15. It was compared to the spectrum of complex 7. An absorption band at $1847 \mathrm{~cm}^{-1}$ in complex 7 was close from the values observed by $\mathrm{P}$. Duan for the $\mathrm{Ni}-\mathrm{H}$ stretching frequencies in dihydride dinuclear complexes. ${ }^{210}$ A broad signal was observed around $1854 \mathrm{~cm}^{-1}$ for complex $\mathbf{1 2}$. Since the intensity of the signal was broad and low in intensity, it was not possible to clearly evaluate if a shift of the absorption band occurred. A broad absorption band was additionally observed at $3458 \mathrm{~cm}^{-1}$ and was attributed to the $\mathrm{NiO}-\mathrm{H}$ stretching frequency.

\subsection{Characterization of an intramolecular dihydrogen bond (DHB).}

In the previous part, the existence of both $\mathrm{NiO}-\mathrm{H}$ and $\mathrm{Ni}-\mathrm{H}$ moieties within complex $\mathbf{1 2}$ were evidenced. This part clarifies if an attraction between the $\mathrm{NiO}-\mathrm{H}$ and $\mathrm{Ni}-\mathrm{H}$ moieties is taking place. Further NMR experiments and additional calculations conducted by A. Romer from professor Mata's group are discussed for this purpose. A NOESY spectrum of complex $\mathbf{1 2}$ is represented in Figure 4.18. A negative correlation peak between the $\mathrm{Ni}-\mathrm{H}$ signal at $-20.5 \mathrm{ppm}$ and the $\mathrm{NiO}-\mathrm{H}$ signal at $-1.8 \mathrm{ppm}$ was observed. This observation likely indicated a spatial interaction of the two hydrogen atoms. 


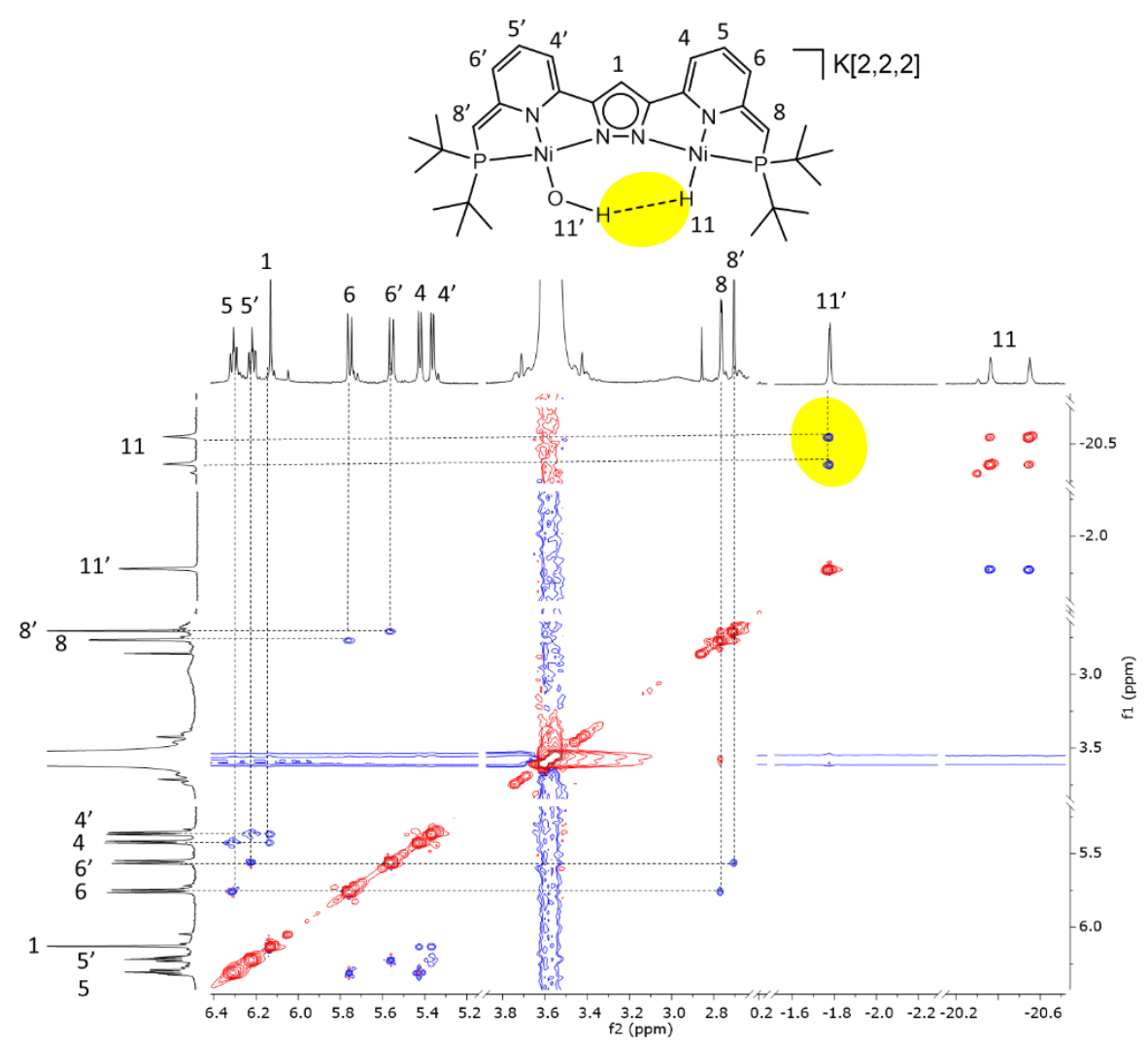

Figure 4.18. NOESY spectrum of complex 12 in THF-d ${ }^{8}$ at $298 \mathrm{~K}$.

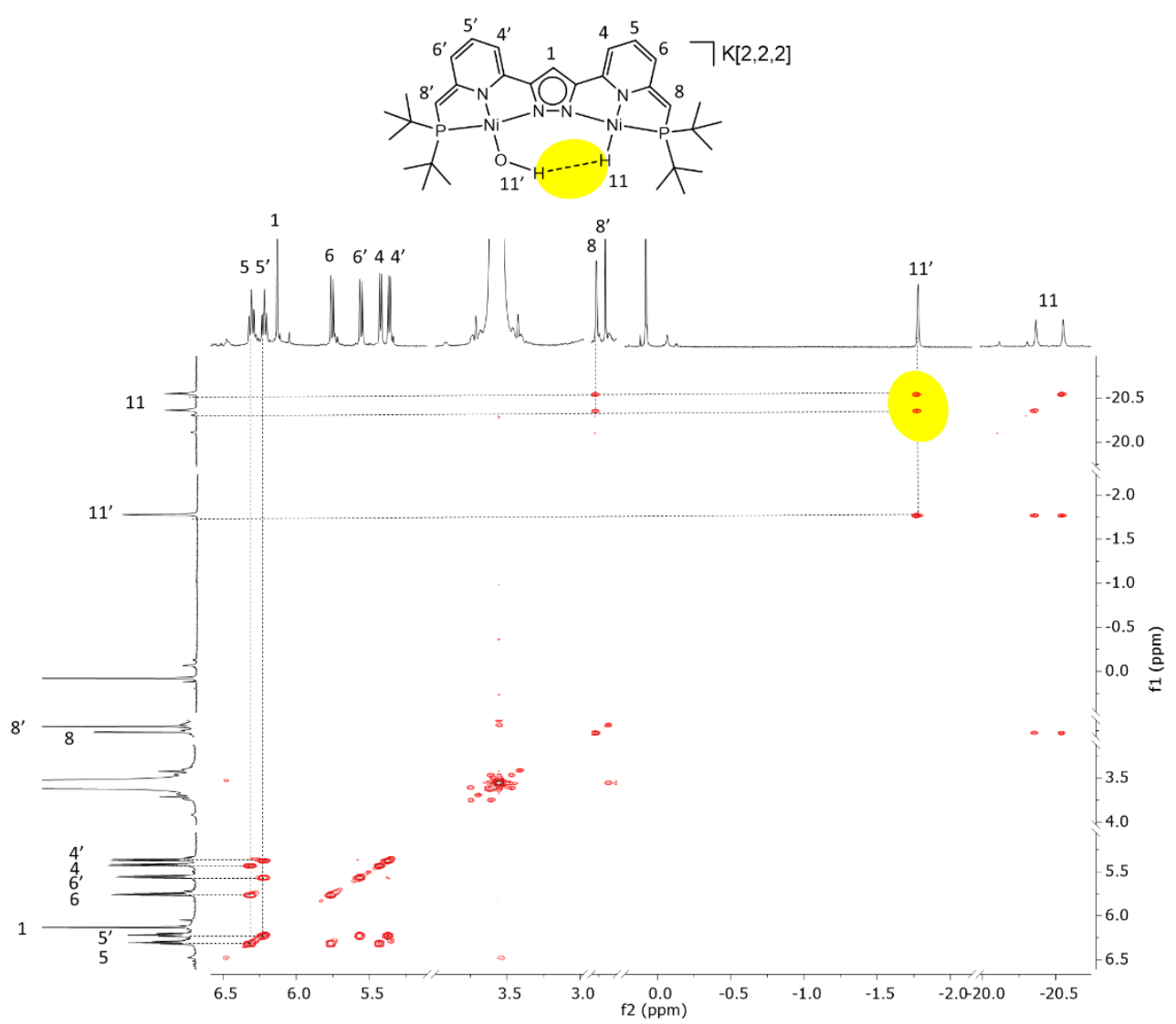

Figure 4.19. COSY spectrum of complex 12 in THF-d ${ }^{8}$ at $298 \mathrm{~K}$. 
The NOE effect specifically indicates the dipolar interaction of two nuclei through space. Thus, the observation of a correlation between the two hydrogen atoms of the $\mathrm{Ni}-\mathrm{OH}$ and $\mathrm{Ni}-\mathrm{H}$ moieties in complex $\mathbf{1 2}$ was reasonable, given the tendency of terminal ligands in pyrazolate based dinuclear complexes to involve steric repulsions (cf chapter 1). COSY NMR spectroscopy was generally used to identify protons which were interacting through chemical bonds. In this respect, it was surprising to observe a correlation between $\mathrm{Ni}-\mathrm{OH}$ and $\mathrm{Ni}-\mathrm{H}$ (Figure 3.17). This result suggested more than dipolar interactions between these two protons.

A NOESY measurement was utilized in order to estimate the distance between the two hydrogen atoms. Such experiment required the following conditions: first, a relaxation delay was set to $3 \mathrm{~s}$. Mixing times were set to 100 and 250 ms respectively. An integral analysis of these 2D NMR spectra confirmed a linear dependence between NOE signals intensity and mixing times. Thus, the distance between two protons $\mathrm{X}$ and $\mathrm{Y}$ could be estimated from a reference distance between two other protons $\mathrm{A}$ and $\mathrm{B}$ according to the following equation:

$$
\frac{\mathrm{I}_{\mathrm{AB}}}{\mathrm{I}_{\mathrm{XY}}}=\left(\frac{\mathrm{d}_{\mathrm{XY}}}{\mathrm{d}_{\mathrm{AB}}}\right)^{6}
$$

Where $I_{A B}$ and $I_{X Y}$ were the integrals of correlation peaks and $d_{A B}$ and $d_{X Y}$ are the distances between the two pairs of protons. In the case of complex 12, the reference distance between the two $\mathrm{C}-\mathrm{H}$ hydrogen atoms 5 and 8 of the backbone was estimated from the X-ray structure to be $2.657 \AA$. Thus, the calculated distance between the two hydrogens of the $\mathrm{NiO}-\mathrm{H}$ and $\mathrm{Ni}-\mathrm{H}$ gave $2.17 \AA$.

A series of NMR measurements at different temperatures were performed in order to determine the relaxation time for the $\mathrm{Ni}-\mathrm{H}$ moieties in complex $\mathbf{7}$ and $\mathbf{1 2}$. The inversion recovery method allowed to obtain the $T_{1}$ values. After exponential fitting, the results were obtained with less than $4 \%$ error. The plot of $\log \left(\mathrm{T}_{1}\right)$ against $1000 / \mathrm{T}$ was represented in Figure 4.18. $\mathrm{T}_{1 \text { min }}$ has been found at $238 \mathrm{~K}$ for a value of $646 \mathrm{~ms}$ in complex 7 and at $258 \mathrm{~K}$ for a value of $224 \mathrm{~ms}$ in complex 12. Thus, the relaxation time of the $\mathrm{Ni}-\mathrm{H}$ in complex $\mathbf{1 2}$ was three-fold lower than the one in complex 7. Both complexes were measured in THF- $\mathrm{d}^{8}$ and involved the same counterions. In those complexes, the proton-proton dipole-dipole interaction was a major contribution for the ${ }^{1} \mathrm{H}$ relaxation rate. The nuclei of the backbone $\left({ }^{13} \mathrm{C},{ }^{31} \mathrm{P},{ }^{14} \mathrm{~N},{ }^{15} \mathrm{~N}\right)$ that could contribute to the relaxation of the $\mathrm{Ni}-\mathrm{H}$ signals were in the same disposition in complex $\mathbf{7}$ or $\mathbf{1 2}$. The hydridenickel dipolar coupling was negligible as the abundance of ${ }^{61} \mathrm{Ni}(\mathrm{S}=3 / 2)$ was very low $(1.1 \%)$. Thus, the large decrease in $\mathrm{T}_{1}$ values for the $\mathrm{Ni}-\mathrm{H}$ signal of complex $\mathbf{1 2}$ at different temperatures could only be explained by the proximity of the ${ }^{1} \mathrm{H}$ nucleus contained in the $\mathrm{NiO}-\mathrm{H}$ moiety. The $\mathrm{T}_{1 \text { min }}$ value of a metal-hydrogen bond can also be used to estimate the distance between hydrogen atoms (notably in $\mathrm{Ni}^{\mathrm{II}}\left(\eta^{2}-\mathrm{H}_{2}\right)$ adducts) according to the following equation: ${ }^{204,205,211,212}$

$$
\mathrm{d}_{\mathrm{H}-\mathrm{H}}=5.815\left(\frac{\mathrm{T}_{1 \mathrm{~min}}}{\mathrm{v}}\right)^{1 / 6}
$$


Using the above equation gave a value of $1.69 \AA$ for the distance between the hydrogen atoms of $\mathrm{NiO}-\mathrm{H}$ and $\mathrm{Ni}-\mathrm{H}$.
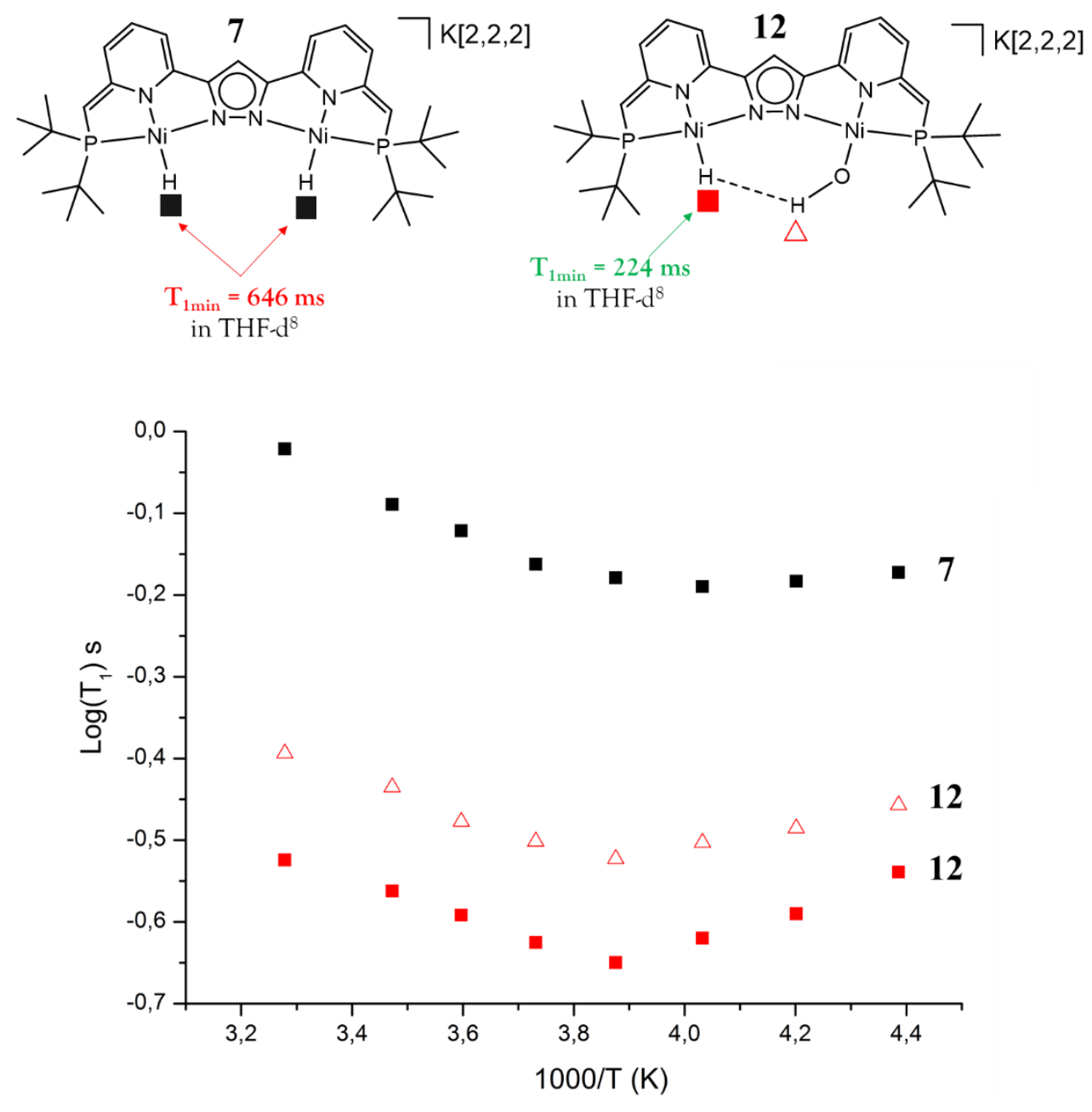

Figure 4.20. $\log \left(\mathrm{T}_{1}\right)$ of complex $\mathbf{7}$ and $\mathbf{1 2}$ ploted against 1000/T.

DFT calculation were performed by A. Romer (group of professor Mata) in order to determine the rotational barrier of the hydroxyl unit around the $\mathrm{Ni}-\mathrm{O}$ bond. These results were shown in Figure 4.21. The most stable configuration was obtained when the dihedral angle $\mathrm{H}-\mathrm{O}-\mathrm{Ni}-\mathrm{N}$ was in eclipsed conformation (conformer A, Figure 4.21). This conformation was precisely where the hydrogen of the $\mathrm{NiO}-\mathrm{H}$ moiety was the closest from the $\mathrm{Ni}-\mathrm{H}$ moiety. The calculated interatomic distance between the two hydrogen atoms was $1.59 \AA$ (Figure 4.22). The rotation of the hydroxyl ligand around the $\mathrm{Ni}-\mathrm{O}$ bond led to an increase in energy. It reached a maximum of $37 \mathrm{~kJ} \cdot \mathrm{mol}^{-1}$ with an angle of $90^{\circ}$. With $180^{\circ}$, the $\mathrm{NiO}-\mathrm{H}$ was directed on the opposite side of the $\mathrm{Ni}-\mathrm{H}$ and a local minimum at $30 \mathrm{kj} \cdot \mathrm{mol}^{-1}$ was found. The steric repulsion between the $\mathrm{tBu}$ groups and the hydroxyl unit could reflect this increase in energy. However, the relatively short $\mathrm{H} \cdots \mathrm{H}$ calculated distance between $\mathrm{NiO}-\mathrm{H}$ and $\mathrm{Ni}-\mathrm{H}$ in the global minimum structure $\mathbf{A}$ suggested an attractive interaction between the two hydrogen atoms. 


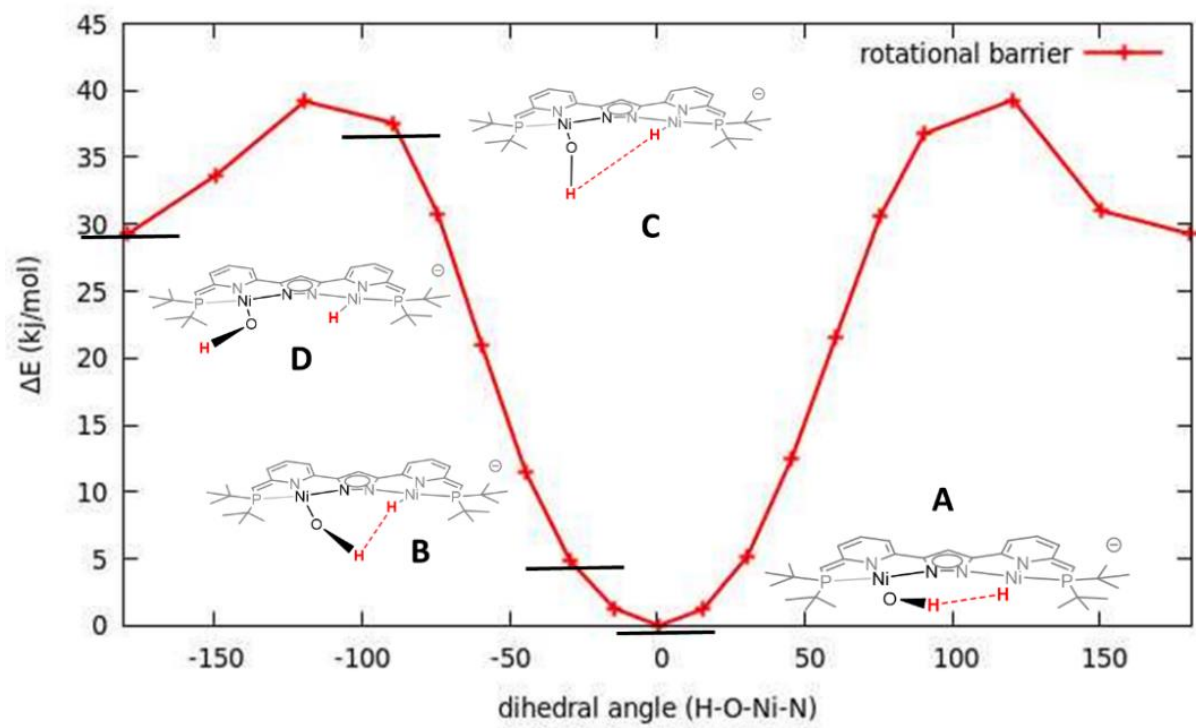

Figure 4.21. DFT calculated energy profile (BP86 / TZVP) for the variation of the $\mathrm{H}-\mathrm{O}-\mathrm{Ni}-\mathrm{N}$ dihedral angle in complex 12. The calculation has been performed in Mata group.

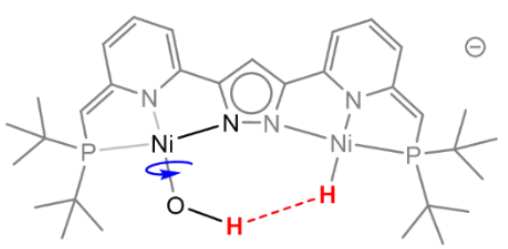

\begin{tabular}{|c|c|}
\hline $\begin{array}{c}\text { Calculated } \\
\mathrm{d}(\mathrm{H} \cdots \mathrm{H})\end{array}$ & $\begin{array}{l}\text { Dihedral } \\
\text { Angle }\left({ }^{\circ}\right)\end{array}$ \\
\hline $3.18 \AA ̊$ & 180 \\
\hline $2.23 \AA ీ$ & 90.5 \\
\hline 1.67 Á & 45.5 \\
\hline $1.59 \AA$ & 0.5 \\
\hline
\end{tabular}

Figure 4.22. Calculated distances between the two hydrogen atoms in red in function of the $\mathrm{H}-\mathrm{O}-\mathrm{Ni}-\mathrm{N}$ dihedral angle in complex 12. The calculation has been performed in Mata group.

The data collected from the NOESY spectra, the $T_{1}$ plot, and the calculations gave various $\mathrm{H} \cdots \mathrm{H}$ interatomic distance between $\mathrm{NiO}-\mathrm{H}$ and $\mathrm{Ni}-\mathrm{H}$. It was possible to rationalize those differences by considering the temperature of the different NMR measurements: the NOESY spectra were recorded at $298 \mathrm{~K}$ and the $\mathrm{T}_{1 \min }$ was measured at $258 \mathrm{~K}$. Thus, the Boltzmann distribution between the different conformers proposed in Figure 4.21 was likely different in the different experiment. At low temperature, the hydroxyl unit likely rotated less, so that the average $\mathrm{H} \cdots \mathrm{H}$ distance was possibly smaller than the one calculated by NOESY at $298 \mathrm{~K}$. Even if the theoretical and experimental values were divergent and an explanation could accommodate those difference, all $\mathrm{H} \cdots \mathrm{H}$ distances evidenced by experiment or DFT were less than $2.4 \AA$, i.e. less than the sum of the Van der Waals radii of two hydrogen atoms. It was assumed that an attractive interaction between those atoms (the dihydrogen bond) would lead to this shorter distance. ${ }^{181}$

In respect to typical parameters of a DHB listed in Figure 4.11: complex 12 displayed an upfield shift of the ${ }^{1} \mathrm{H}$ NMR hydride signal $\left(\Delta \delta=0.25 \mathrm{ppm}\right.$, Figure 4.12), a three-fold decrease in $\mathrm{T}_{1 \text { min }}$ (Figure 4.20), and $\mathrm{H} \cdots \mathrm{H}$ interatomic distances $<2.4 \AA$. It suggested the occurrence of a DHB. Additionally, the DHB was supported by COSY experiment (Figure 4.19). An interesting ${ }^{31} \mathrm{P}$ 
NMR spectrum obtained after labelling experiment during the mechanistic investigation was obtained (Figure 4.36). It possibly implicated the difference between the intermolecular $\mathrm{H} \cdots \mathrm{H}$ and $\mathrm{D} \cdots \mathrm{H}$ interaction. This difference resulted in very small chemical shifts $(0.02 \mathrm{ppm})$. It was called "DHB isotopic shift".

\subsection{Mechanistic investigation of the reaction with $\mathrm{H}_{2} \mathrm{O}$.}

In the previous part, it was demonstrated that the reaction of complex $\mathbf{7}$ gave complex $\mathbf{1 2}$ in presence of water. Complex 12 involved an intramolecular DHB. This part was focusing on the identification of intermediates during the hydrolysis as illustrated in Figure 4.23. According to the titration shown in Figure 4.12, two ${ }^{1} \mathrm{H}$ NMR signals for pyrazolate $\mathrm{C}-\mathrm{H}$ signal were observed during the reaction. One corresponded to complex 7, the other to the complex 12. The concentration of the intermediate was so low that its detection was not possible. The hypothesis of an instable $\mathrm{Ni}^{\mathrm{il}}\left(\eta^{2}-\mathrm{H}_{2}\right)$ intermediate (cf discussion in the introduction) resulting from the protonation of $\mathrm{Ni}-\mathrm{H}$ with $\mathrm{H}_{2} \mathrm{O}$ was a possible hypothesis.
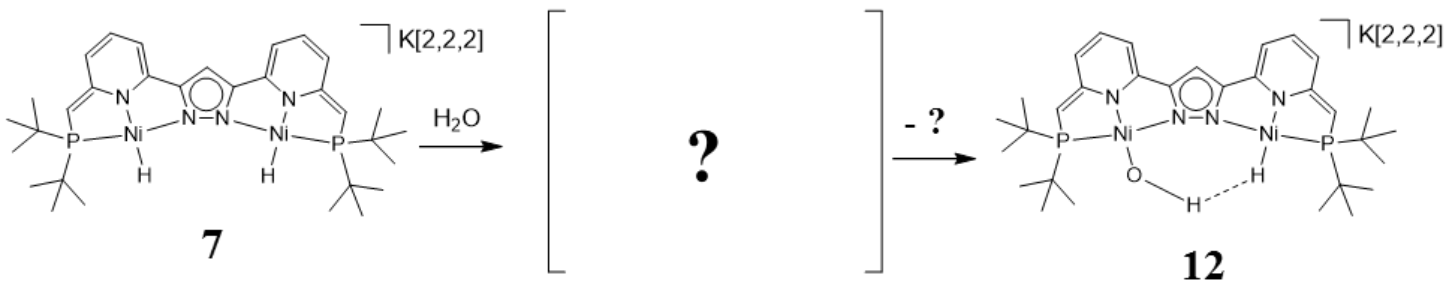

Figure 4.23. Mechanistic problem for the reaction of complex $\mathbf{7}$ to give complex $\mathbf{1 2}$.

The detection of an intermediate was achieved by using the alternative complex 7B for the hydrolysis (Figure 4.24). Complex 7B was obtained by the same synthetic procedure described for complex 7 (Figure 3.4). However, the procedure was stopped before the addition of the $[2,2,2]$ cryptand. The cryptand was a necessary element for the crystallization and the full characterization of the complex, but not for the inherent stability of the anionic dihydride complex. The ${ }^{1} \mathrm{H}$ and ${ }^{31} \mathrm{P}$ NMR spectroscopic properties of complex 7B were very similar to complex 7 (compare Figure 4.25 with Figure 3.7). Complex 7B displayed a single ${ }^{31} \mathrm{P}$ NMR signal at $85.2 \mathrm{ppm}$, which was slightly downfield shifted compared to complex 7 (84.1 ppm). The presence of a single ${ }^{31} \mathrm{P}$ NMR signal confirmed the purity of the sample.
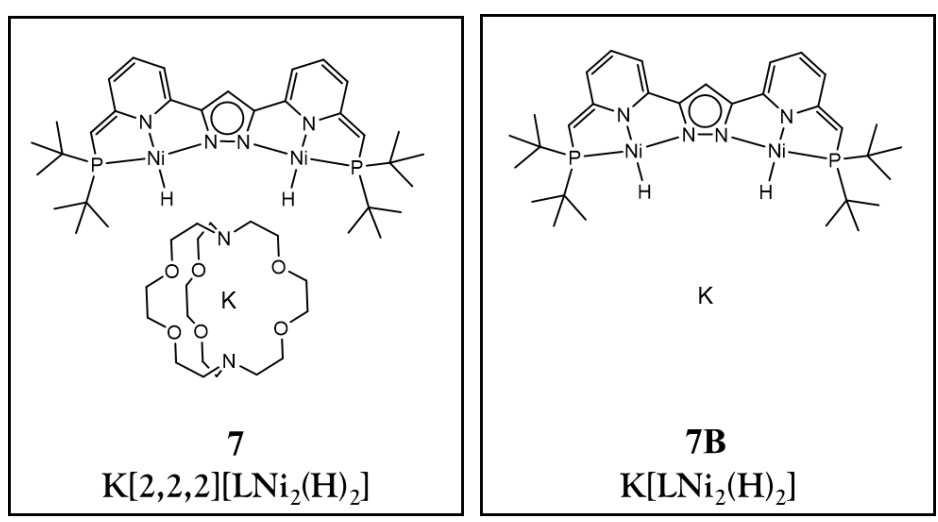

Figure 4.24. Two different complexes for the study of hydrolysis with water. 


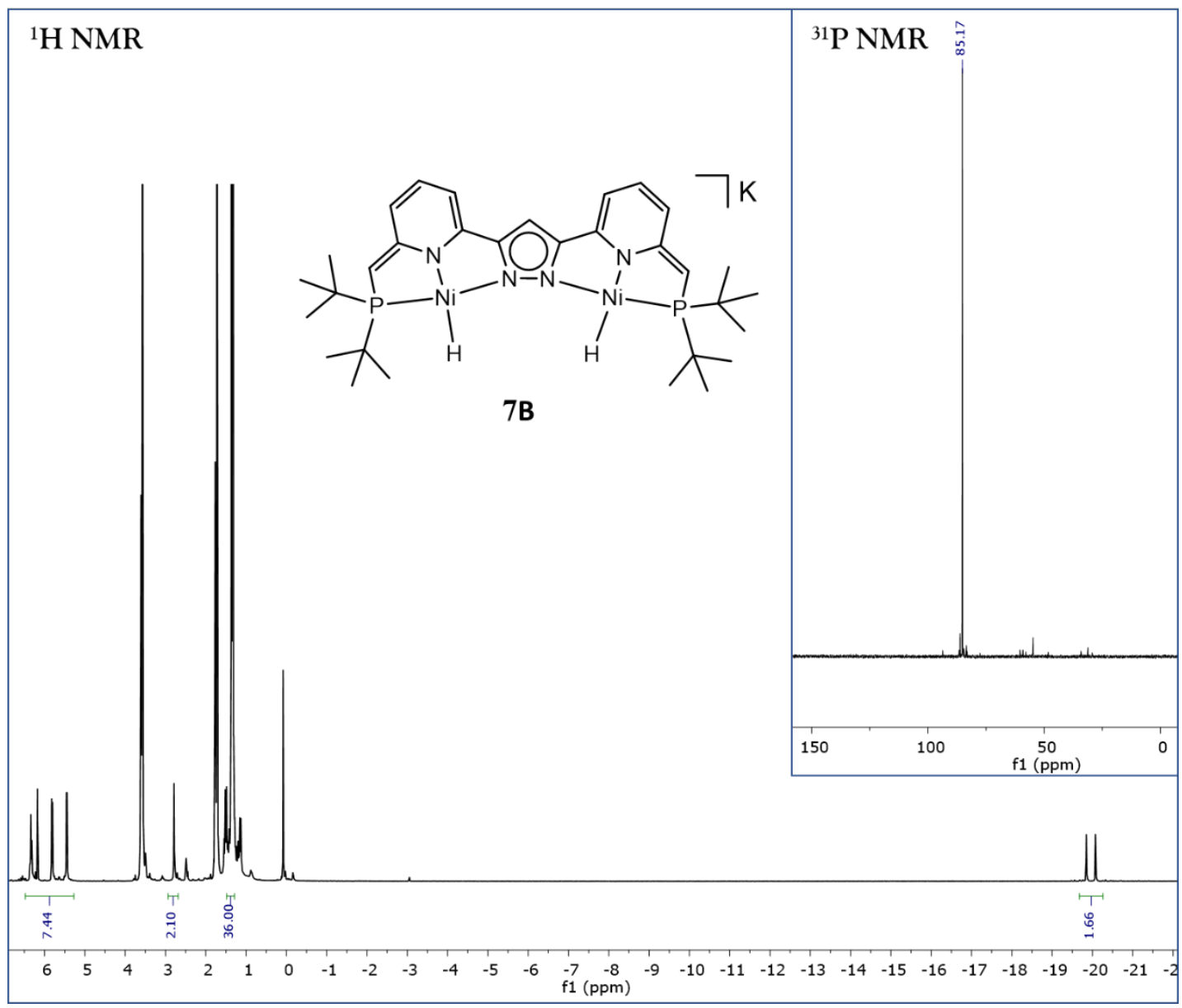

Figure 4.25. ${ }^{1} \mathrm{H}$ and ${ }^{31} \mathrm{P}$ NMR (top right) spectra of complex 7B in THF-d ${ }^{8}$.

Complex 7B (Figure 4.24) was used for the mechanistic analysis of the reaction with $\mathrm{H}_{2} \mathrm{O}$. One equivalent of $\mathrm{H}_{2} \mathrm{O}$ was added to an NMR sample of complex 7B dissolved in THF-d ${ }^{8}$ at $258 \mathrm{~K}$. A colour change from dark violet to dark brown was observed. The temperature was kept at $258 \mathrm{~K}$ for the NMR measurement. A colour change was not observed in the case of complex $\mathbf{7}$ with $\mathrm{H}_{2} \mathrm{O}$. The ${ }^{1} \mathrm{H}$ and ${ }^{31} \mathrm{P}$ NMR spectra of this experiment were represented in Figure 4.26 and in Figure 4.28 respectively.

New ${ }^{1} \mathrm{H}$ NMR signals were observed and clearly indicated the formation of a new product. A single signal for a pyrazole $\mathrm{C}-\mathrm{H}$ was detected at $6.60 \mathrm{ppm}$. Three downfield signals at $7.81 \mathrm{ppm}$, $7.42 \mathrm{ppm}$ and $7.26 \mathrm{pmm}$ were consistent with protons of an aromatic pyridine (compared with complex 2, chapter 1). Three other upfield signals (6.33 ppm, $5.83 \mathrm{ppm}$ and $5.46 \mathrm{ppm}$ ), were consistent with protons of a dearomatized pyridine (cf chapter 2). Two hydride signals in the upfield region were detected. The hydride signals and the signals in the aromatic region were almost equally integrating for 1 . The - $\mathrm{CH}$ signal of a deprotonated side arm was found at 2.81 ppm. A signal for a $-\mathrm{CH}_{2}$ group was overlapping with the signal of the solvent. It was indirectly detected by a NOESY correlation (Figure 4.27, yellow shaded area). As a link between protonation and aromaticity of the pyridine, the $-\mathrm{CH}_{2}$ group correlated with the proton of the aromatic pyridine (yellow shaded area), while the - $\mathrm{CH}$ group correlated with the proton of the dearomatized pyridine (green shaded area). 


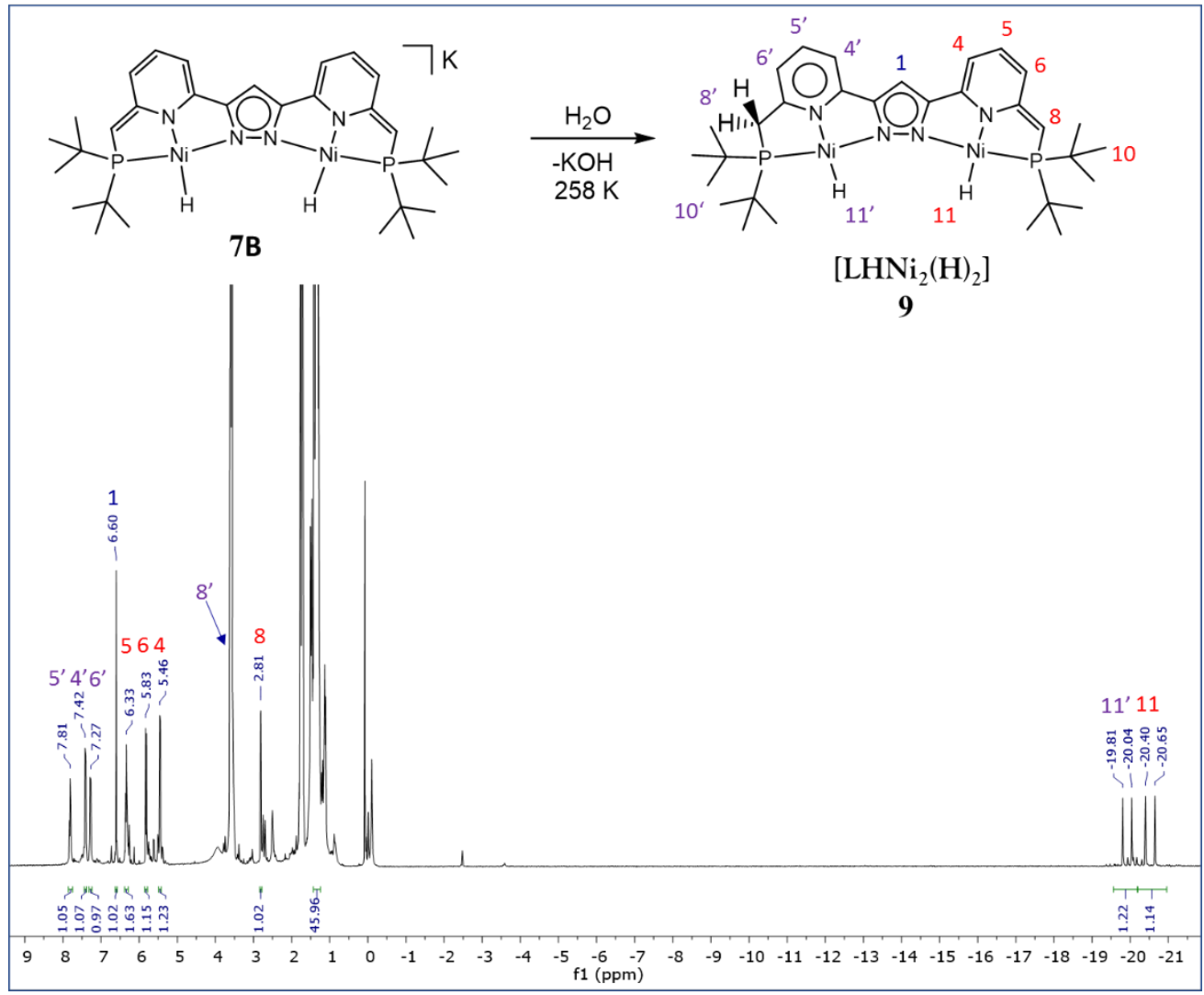

Figure 4.26. ${ }^{1} \mathrm{H}$ NMR spectrum obtained after addition of $\mathrm{H}_{2} \mathrm{O}$ to complex 7B resulting in the formation of complex 9 in $\mathrm{THF}^{8}{ }^{8}$ at $258 \mathrm{~K}$

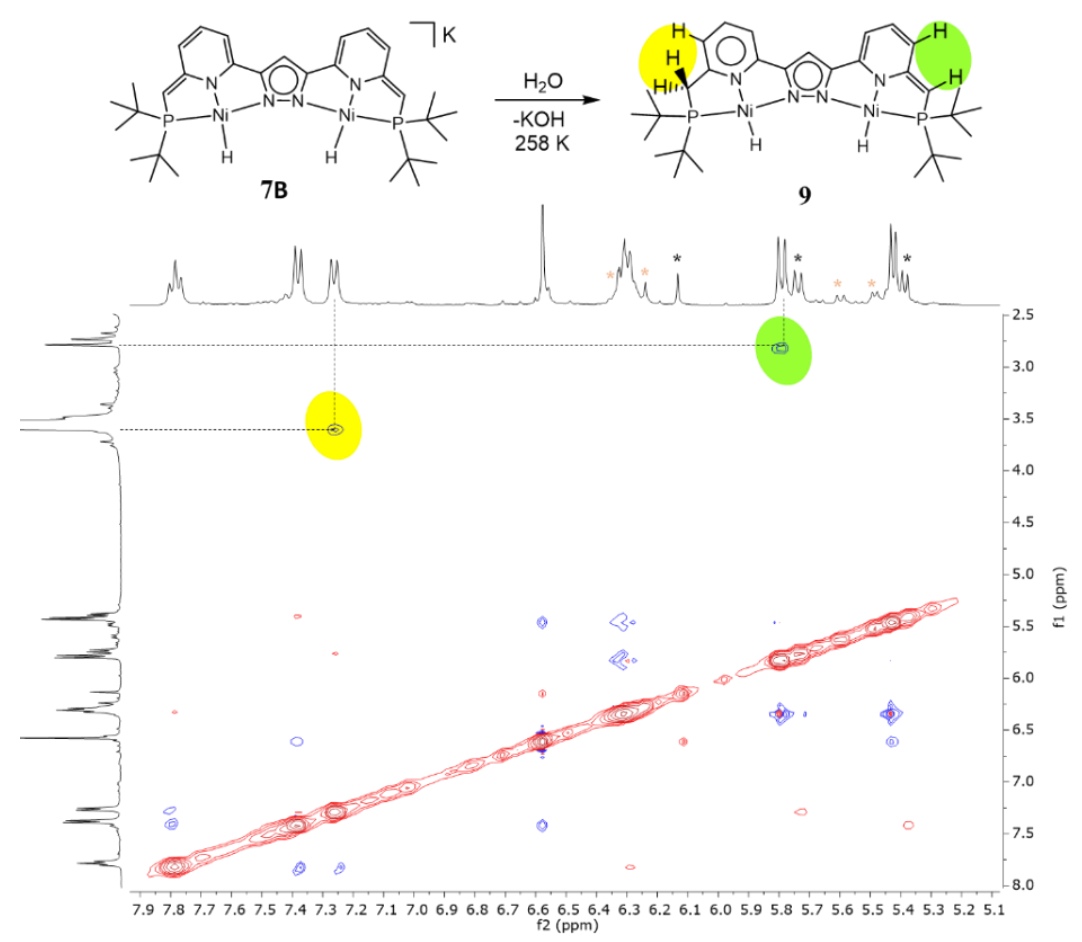

Figure 4.27. NOESY NMR spectrum obtained after addition of $\mathrm{H}_{2} \mathrm{O}$ to complex 7B resulting in the formation of complex 9 in THF-d $^{8}$ at $258 \mathrm{~K}$. Black asterix represented an uncomplete consumption of $\mathbf{7 B}$. 


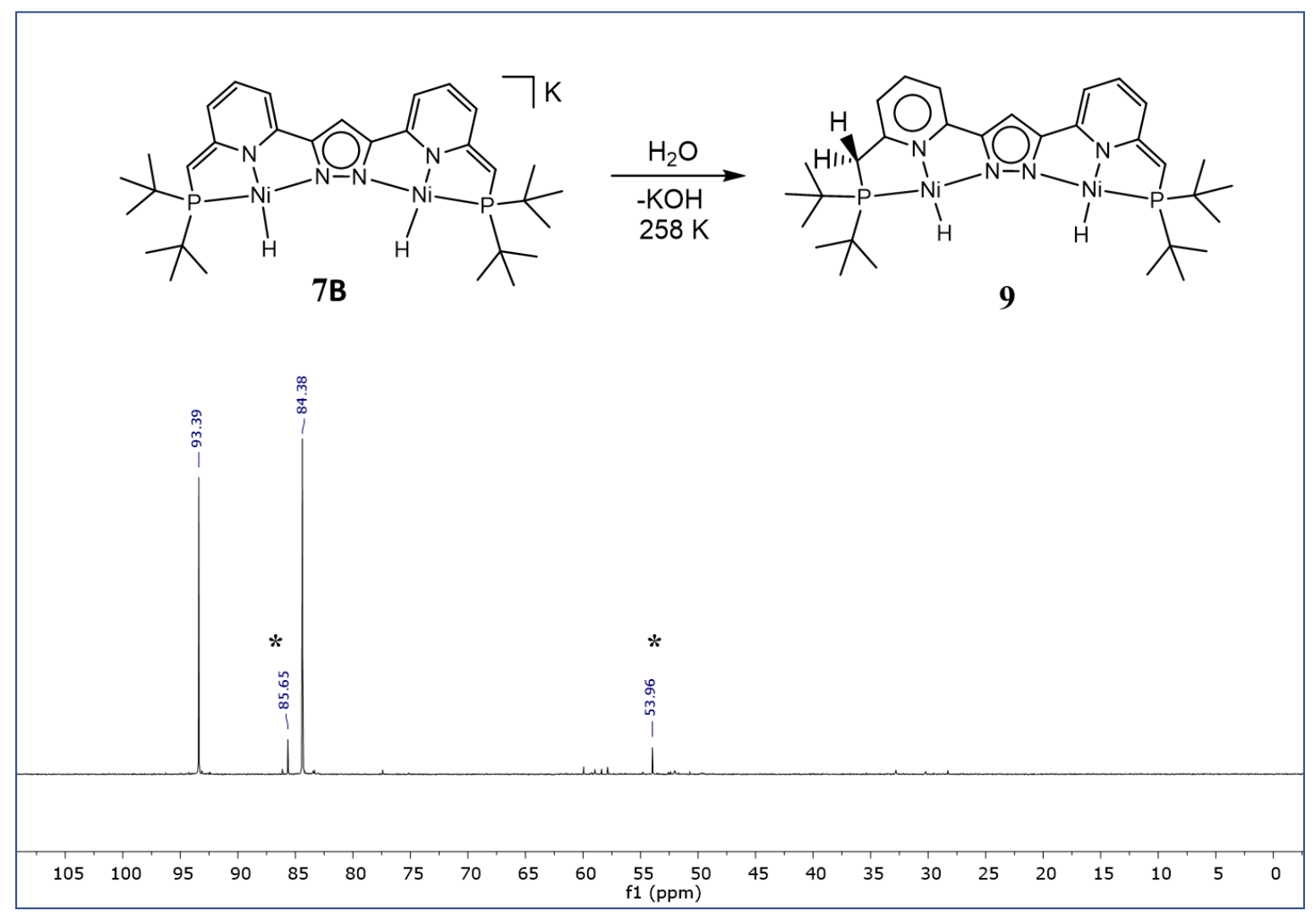

Figure 4.28. ${ }^{31} \mathrm{P}$ NMR spectrum obtained after addition of $\mathrm{H}_{2} \mathrm{O}$ to complex 7B resulting in the formation of complex 9 in THF-d $\mathrm{d}^{8}$ at $258 \mathrm{~K}$. Black asterisk indicated the formation of complex 12.

The ${ }^{1} \mathrm{H}$ NMR experiment suggested the formation of the asymmetric complex $\mathbf{9}$. Complex 9 carried one proton more than complex 7B. This proton was located on the $-\mathrm{CH}_{2}$ group. The protonation of the side arm in complex 7B led to rearomatization of the corresponding pyridine, which induced dramatic NMR changes for all nuclei. Two main ${ }^{31} \mathrm{P}$ NMR signals were observed on the spectrum shown in Figure 4.28 and confirmed this hypothesis. The two NMR signals were having the same intensity. The signal at $84.4 \mathrm{ppm}$ was similar to the signal at $85.2 \mathrm{ppm}$ in complex 7B (Figure 4.26). The other signal was observed at $93.4 \mathrm{ppm}$. The deshielding effect seen in the second ${ }^{31} \mathrm{P}$ NMR signal was likely induced by the rearomatization of the pyridine (positive inductive effect).

Complex 9 was not further analysed as it was only stable in solution at $258 \mathrm{~K}$. The spectroscopic data collected from those experiments was not enough to confirm the structure of complex $\mathbf{9}$. However, NMR clearly evidenced the presence of an asymmetric intermediate featuring two inequivalent hydrides. The asymmetry was likely coming from one side arm of the ligand being protonated. Even if the acidity of water was likely low in THF, it appeared reasonable that the $\mathrm{CH}$ arm in complex 7B was basic enough to deprotonate $\mathrm{H}_{2} \mathrm{O}$. This reaction gave rise to complex 9 likely with formal $\mathrm{KOH}$ elimination. Eventually, the first step of the reaction mechanism for the formation of complex $\mathbf{9}$ to $\mathbf{1 2}$ was proposed (Figure 4.29). 

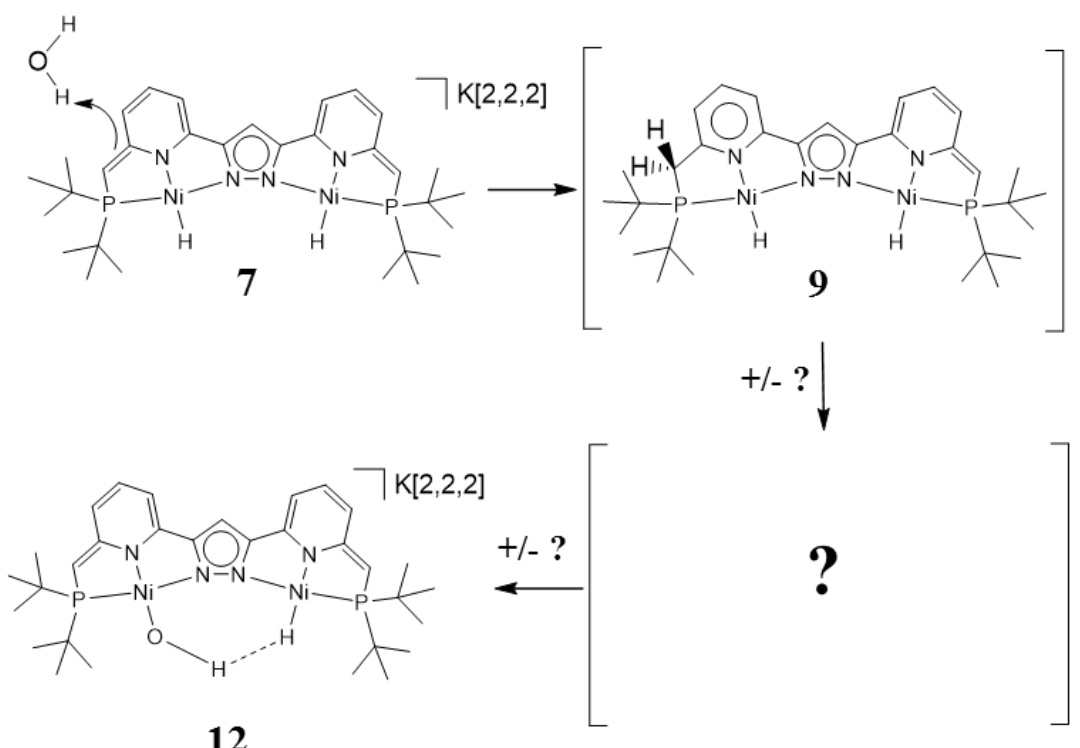

12

Figure 4.29. New mechanistic problem for the reaction of complex 9 to complex $\mathbf{1 2 .}$

Complex 9 was stable at $258 \mathrm{~K}$ but further reacted at $278 \mathrm{~K}$. The transformation of complex 9 was monitored by ${ }^{1} \mathrm{H}$ and ${ }^{31} \mathrm{P}$ NMR spectroscopy at $278 \mathrm{~K}$. When the temperature of the sample was increased from $258 \mathrm{~K}$ to $278 \mathrm{~K}$, new ${ }^{1} \mathrm{H}$ NMR signals slowly formed. Several intermediates were probably involved in the reaction, but their concentration was too low, and their identification remained difficult. A ${ }^{1} \mathrm{H}$ NMR signal at $4.55 \mathrm{ppm}$ was detected which was likely due to formation of $\mathrm{H}_{2}$. A major complex formed after $665 \mathrm{~min}$ and displayed very similar ${ }^{1} \mathrm{H}$ and ${ }^{31} \mathrm{P}$ NMR signals (Figure 4.31 respectively Figure 4.32) compared to complex 12 (cf part 4.2). Thus, it was possible that complex 12B formed. Complex 12B could not be crystalized and the characterization of the complex was not further pursued. Complex $\mathbf{1 2}$ and complex 12B were different from each other by a [2,2,2] cryptand molecule. Complex $\mathbf{7}$ led to complex $\mathbf{1 2}$ in presence of water. It was suggested that complex 7B led to complex 12B in presence of water. When the NMR sample was removed from the NMR spectrometer, the colour of the solution was no more brown but bordeaux, akin to complex $\mathbf{1 2}$.

In conclusion the monitoring by NMR spectroscopy of the reaction of complex 9 to give complex 12 could not evidence a clear mechanistic pathway. The last step of the reaction was of critical interest for this work as it formed $\mathrm{H}_{2}$. To be transformed into complex 12, complex $\mathbf{9}$ had to eliminate one hydride and one hydrogen on the side arm. It was tempting to suggest that one hydride and one hydrogen of the $-\mathrm{CH}_{2}$ group of complex 9 would combine into $\mathrm{H}_{2}$ with a concomitant dearomatization of a pyridine moiety (Figure 4.30). This possibility will be discussed in the next part with labelling experiments. 


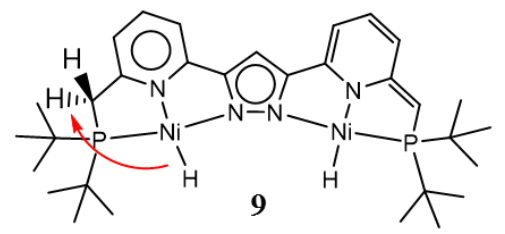

?

Figure 4.30. Mechanstic problem: Did the combination between a hydride of complex 9 and the hydrogen atom of the - $\mathrm{CH}_{2}$ group led to the $\mathrm{H}_{2}$ observed by ${ }^{1} \mathrm{H}$ NMR spectroscopy?

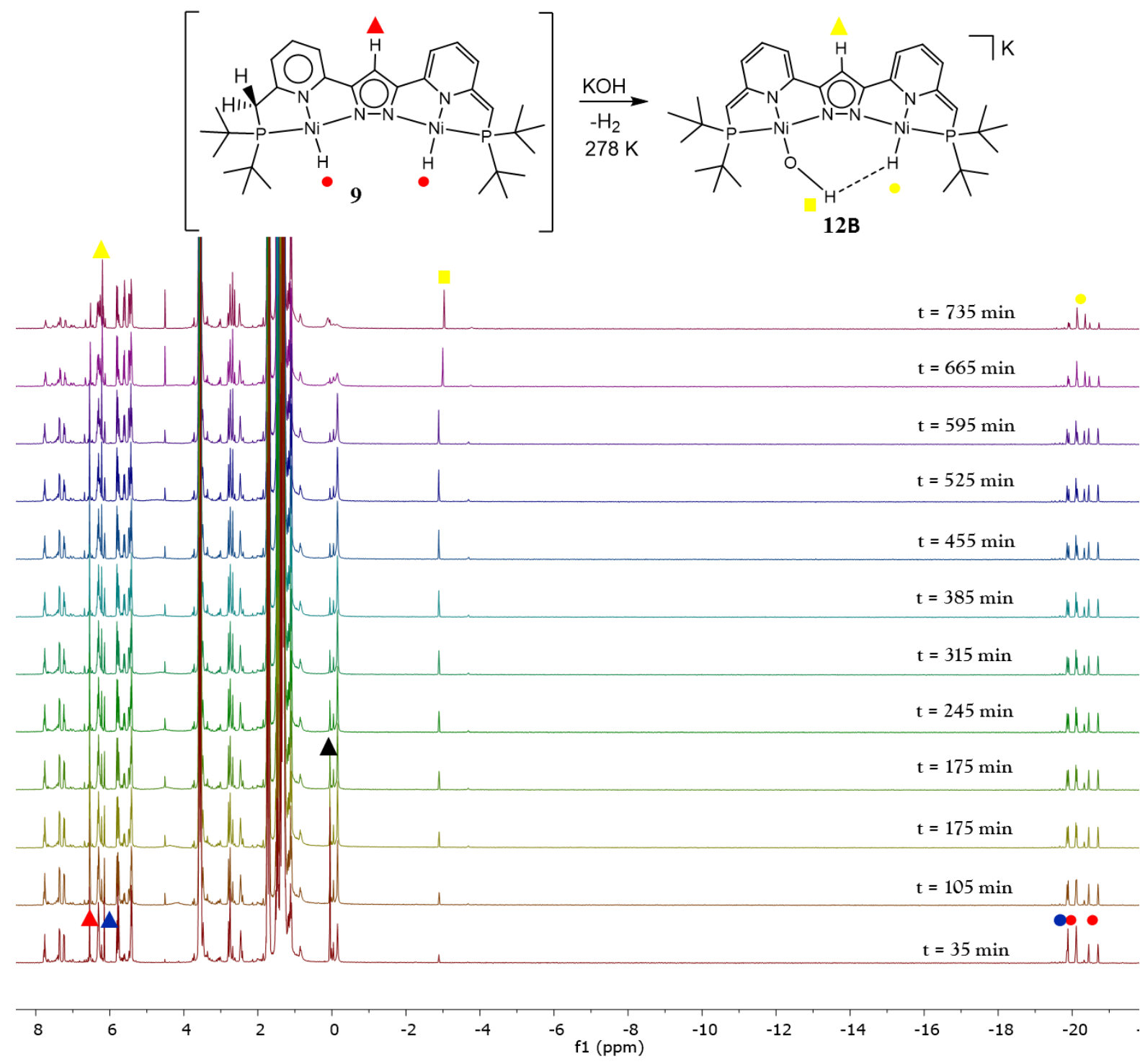

Figure 4.31. Monitoring the reaction of complex 9 en route to 12B by ${ }^{1} \mathrm{H}$ NMR spectroscopy in THF- ${ }^{8}$ at 278 K. $(\boldsymbol{\Delta})$ was correlated to the $-\mathrm{CH}$ resonance of the pyrazole in complex 7B. $(\boldsymbol{\bullet})$ was correlated to the $\mathrm{Ni}-\mathrm{H}$ signal of $\mathbf{7 B}$. ( $\mathbf{(})$ was the signal of silicon grease contained in the deuterated solvent. 

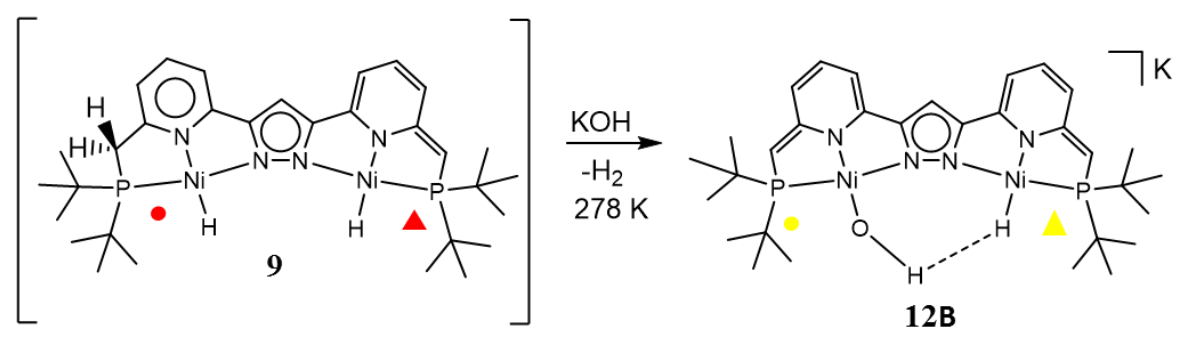

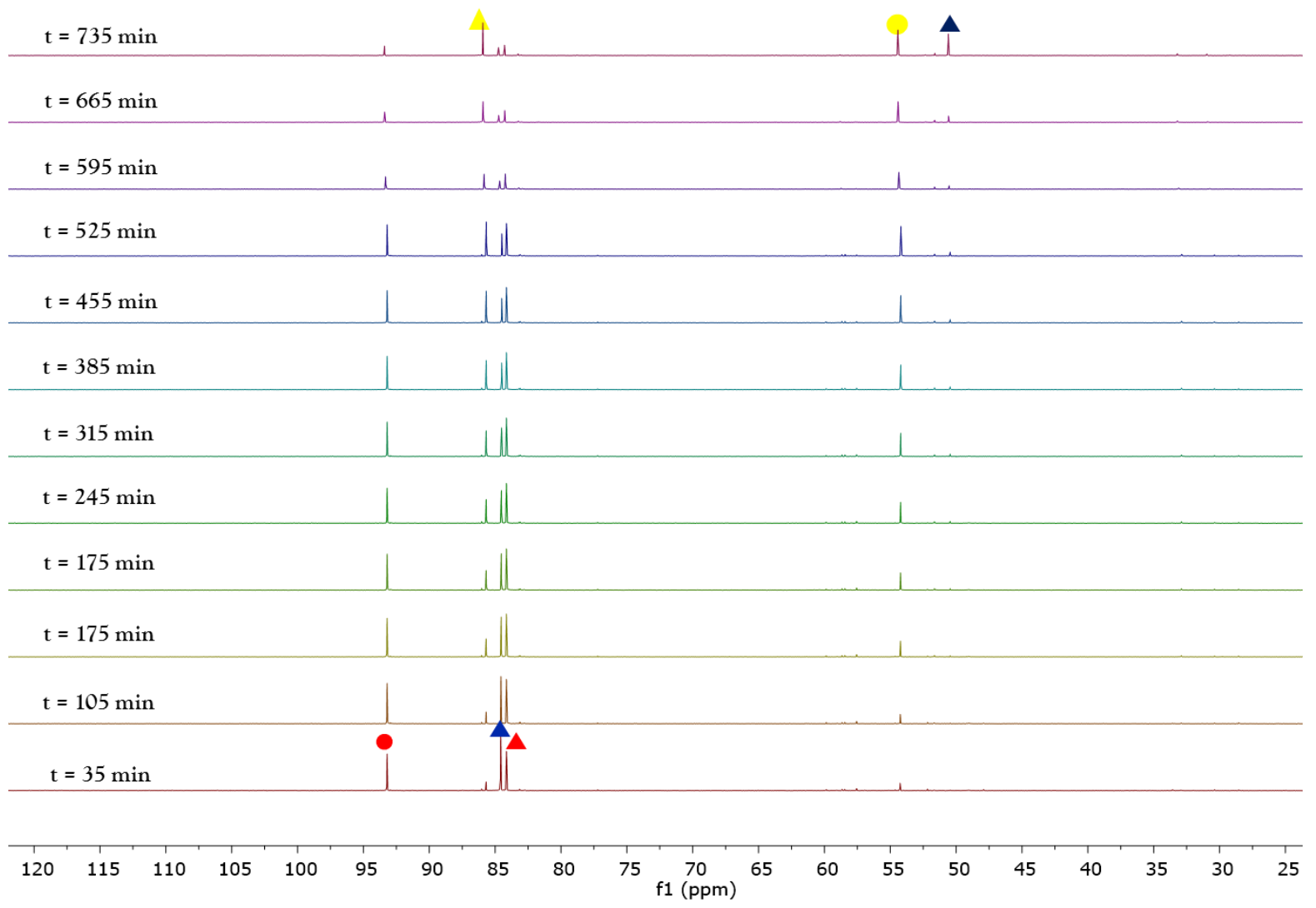

Figure 4.32. Monitoring the reaction of complex 9 en route to $\mathbf{1 2 B}$ by ${ }^{31} \mathrm{P}$ NMR spectroscopy in THF-d ${ }^{8}$ at $278 \mathrm{~K}$. ( $\left.\mathbf{\Delta}\right)$ represented residual amounts of complex $\mathbf{7 B}$ which was not hydrolysed and (৯) represented the formation of complex 11 (Figure 4.42).

\subsection{Labeling experiment with $\mathrm{D}_{2} \mathrm{O}$.}

A similar study as the one described in part 4.4 was conducted however, $\mathrm{H}_{2} \mathrm{O}$ was replaced by $\mathrm{D}_{2} \mathrm{O}$. Adding one equivalent of $\mathrm{D}_{2} \mathrm{O}$ to complex 7 led to a similar ${ }^{1} \mathrm{H}$ NMR spectrum as the one observed in complex 12 (compare Figure 4.33 with Figure 4.14). A ${ }^{1} \mathrm{H}$ NMR signal for the $\mathrm{Ni}-\mathrm{OH}$ moiety $(-1.8 \mathrm{ppm})$ was observed. Deuterated water contained traces of HOD. In respect to the peak intensity of the $\mathrm{Ni}-\mathrm{OH}$ moiety found in the ${ }^{1} \mathrm{H}$ NMR spectrum, this was likely not a sufficient source of hydrogen. No gas $\left(\mathrm{H}_{2}\right.$ or $\left.\mathrm{HD}\right)$ was detected in the NMR sample probably due to the low concentration of the gas. A ${ }^{31} \mathrm{P}$ NMR spectrum revealed a high number of ${ }^{31} \mathrm{P}$ NMR peaks. Instead of having 2 signals, 8 were obtained (Figure 4.34). It was noteworthy to observe the presence of low intensity signals that could correspond to a Ni-D moiety (Figure 4.34). The abnormal number of ${ }^{31} \mathrm{P}$ NMR signals will be explained within Figure 4.38. 


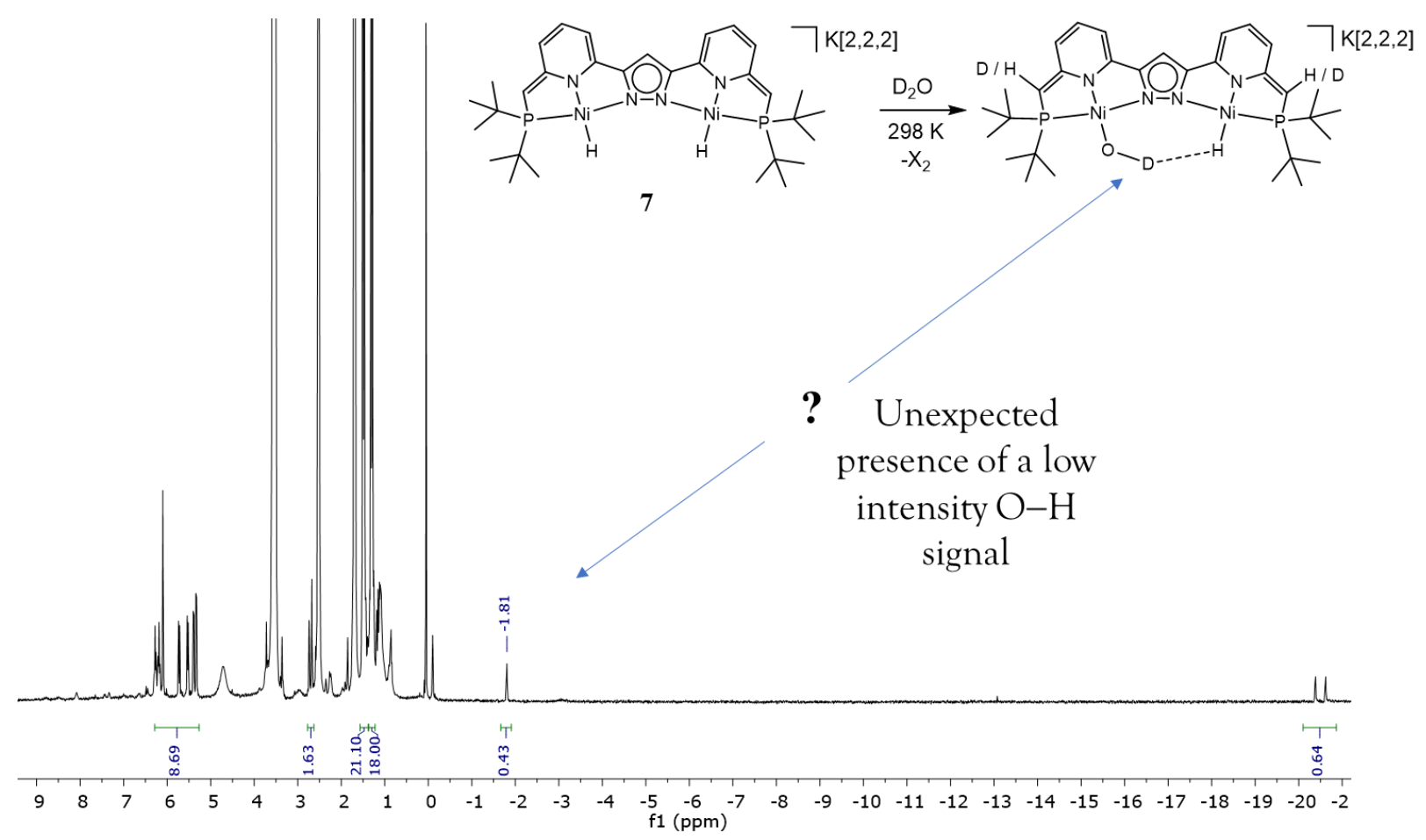

Figure 4.33. ${ }^{1} \mathrm{H}$ NMR spectrum after addition of 1 equivalent $\mathrm{D}_{2} \mathrm{O}$ to complex 7 in THF-d ${ }^{8}$ at $298 \mathrm{~K}$.

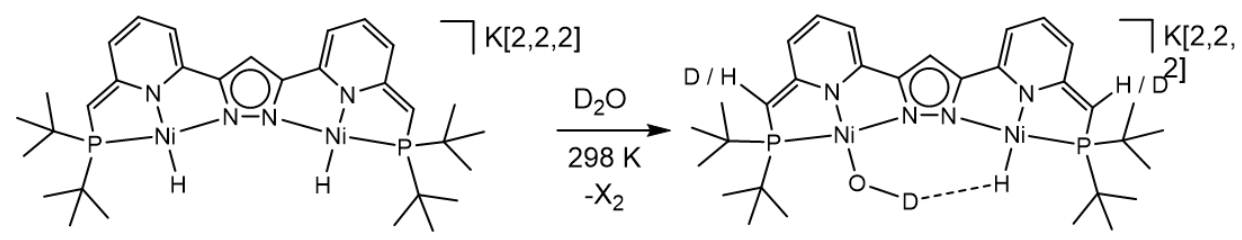

7
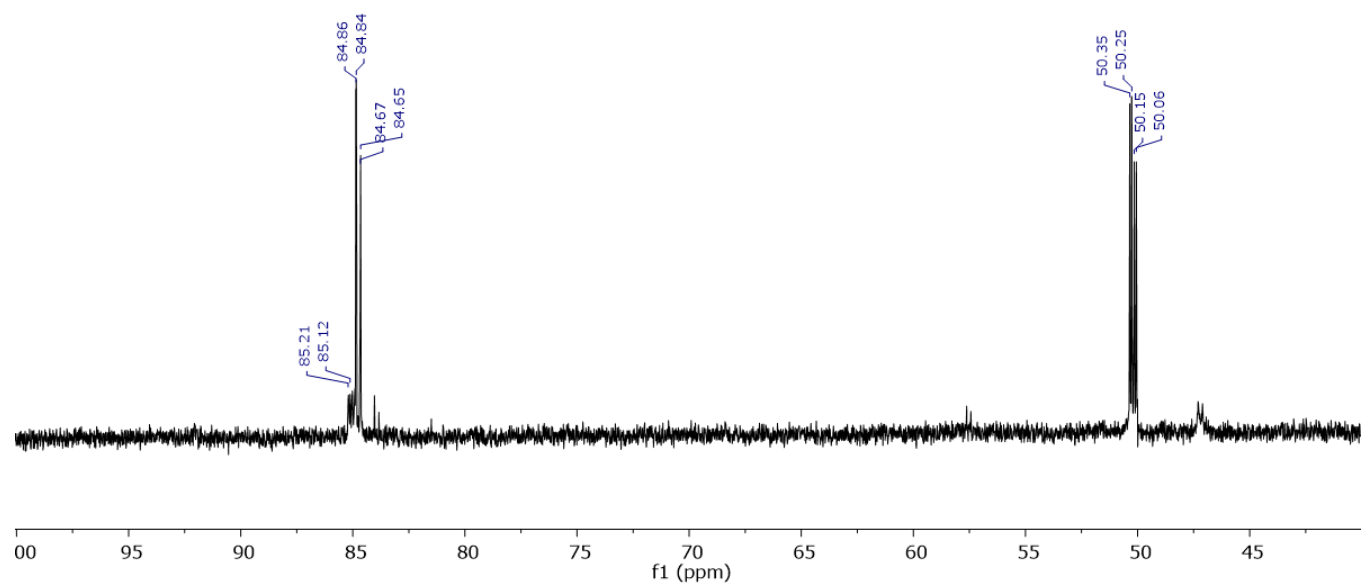

Figure 4.34. ${ }^{31} \mathrm{P}$ NMR spectrum after addition of 1 equivalent $\mathrm{D}_{2} \mathrm{O}$ to complex 7 in THF-d ${ }^{8}$ at $298 \mathrm{~K}$. 
In close analogy with the mechanistic investigation performed in part $4.4, \mathrm{D}_{2} \mathrm{O}$ was added to complex 7B at $258 \mathrm{~K}$. A similar colour change was observed. The deep violet solution turned into deep brown. The sample was kept at $258 \mathrm{~K}$ during the NMR measurement. The resulting ${ }^{1} \mathrm{H}$ NMR spectrum was shown in Figure 4.35. The ${ }^{1} \mathrm{H}$ NMR spectrum was similar to the one observed in Figure 4.26. A ${ }^{1} \mathrm{H}$ NMR signal was observed in the region of the - $\mathrm{CH}$ groups but its intensity was lower in respect to the integral of the - $\mathrm{CH}$ of the pyrazole. It was consistent with the partial deuteration of the side arm. The integral of one hydride signal was equal to 1 . The other hydride signal had the same intensity and it overlapped with the hydride signal of complex 7B still present in solution. Thus, the hydride signals were apparently not affected by the presence of $\mathrm{D}_{2} \mathrm{O}$ at $258 \mathrm{~K}$.

A ${ }^{31} \mathrm{P}$ NMR spectrum was recorded and displayed seven signals (Figure 4.36). Three downfield ${ }^{31} \mathrm{P}$ NMR signals at $93.38 \mathrm{ppm}, 93.12 \mathrm{ppm}$ and $92.85 \mathrm{ppm}$ were observed. The distances between closest signals were $0.26 \mathrm{ppm}(43 \mathrm{~Hz})$. Two pairs of upfield ${ }^{31} \mathrm{P}$ NMR signals at $(84.54 \mathrm{ppm}$ and $84.43 \mathrm{ppm})$ and $(84.37 \mathrm{ppm}$ and $84.16 \mathrm{ppm})$ were recorded. Both were separated by $0.21 \mathrm{ppm}$ $(34 \mathrm{~Hz})$. An isotope shift was likely responsible for the different signals observed. In the downfield region, the phosphorus atom bound to the $-\mathrm{CH}_{2}$ displayed three signals, likely because of three different possible combinations: $-\mathrm{CH}_{2}, \mathrm{CHD}$ and $\mathrm{CD}_{2}$. Each time a ${ }^{2} \mathrm{H}$ atom was bound to the corresponding carbon atom, an upfield isotope shift of $0.26 \mathrm{ppm}$ was observed in ${ }^{31} \mathrm{P}$ NMR spectroscopy. For the -CH group, only two combinations were possible: - $\mathrm{CH}$ or -CD. It was well represented by the pair of two signals in the upfield region. Both were spaced by $0.21 \mathrm{ppm}$. The chemical shifts allowed to recognize the moiety of the different complexes.

This data allowed to acknowledge the existence of an equilibrium between 7B and its products in presence of $\mathrm{D}_{2} \mathrm{O}$ at $258 \mathrm{~K}$.

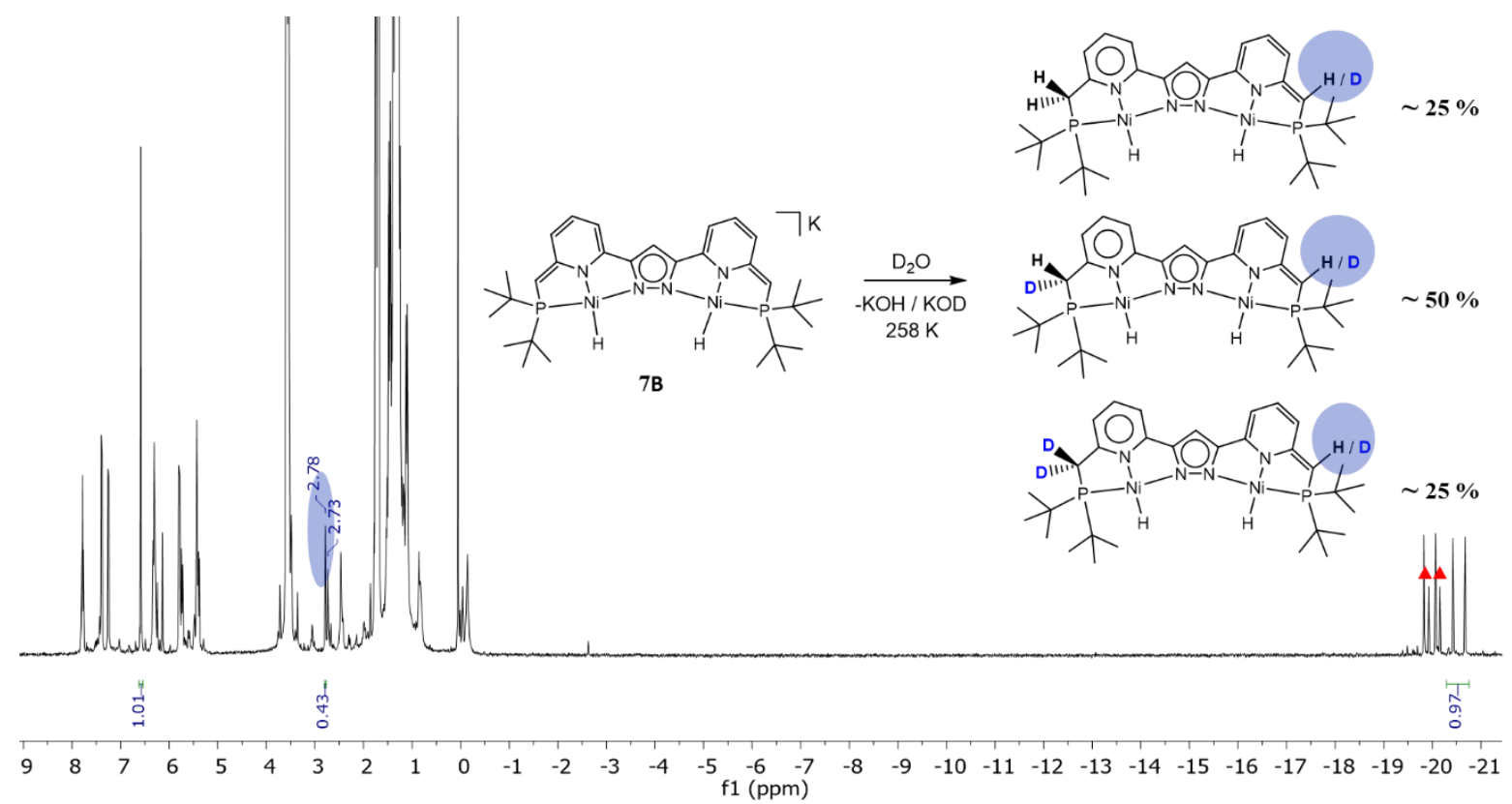

Figure 4.35. ${ }^{1} \mathrm{H}$ NMR spectrum recorded after addition of 1 equivalent $\mathrm{D}_{2} \mathrm{O}$ to complex 7B in $\mathrm{THF}^{8}{ }^{8}$ at $258 \mathrm{~K}$. ( $\left.\boldsymbol{\Delta}\right)$ represented the residual Ni-H signal coming from complex 7B. 


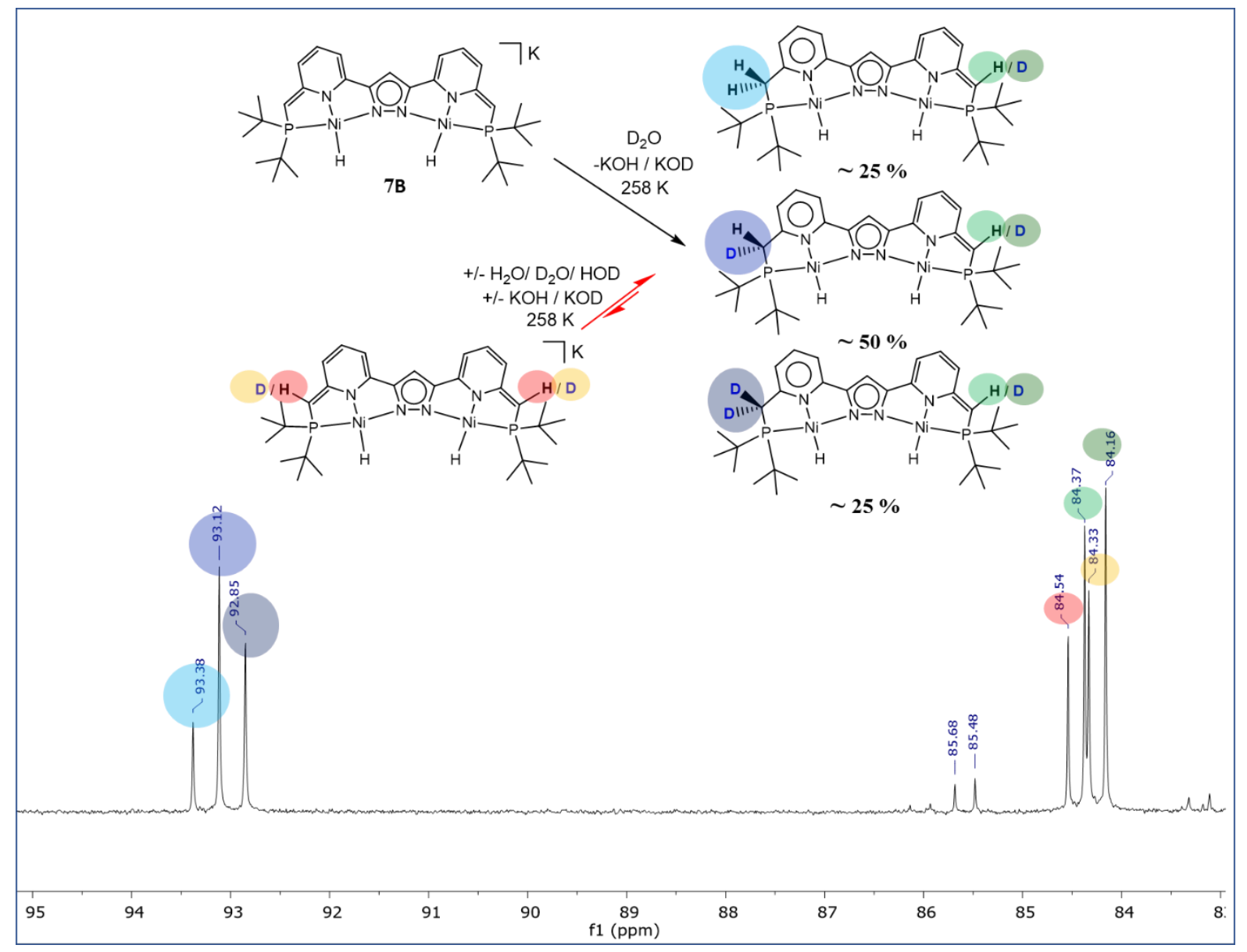

Figure 4.36. ${ }^{31} \mathrm{P}$ NMR spectrum after addition of 1 equivalent $\mathrm{D}_{2} \mathrm{O}$ to complex 7B in THF$\mathrm{d}^{8}$ at $258 \mathrm{~K}$.

The NMR sample was then heated to $298 \mathrm{~K}$, whereby the deuterated mixture of complex $\mathbf{9}$ evolved to a deuterated mixture of complex 12B. This reaction was accompanied by formation of $\mathrm{H}_{2}$ and HD. It was highlighted in yellow in Figure 4.37. The ${ }^{1} \mathrm{H}$ NMR spectrum of the final complexes obtained after the consumption of complex $\mathbf{9}$ had a limited interest in terms of mechanistic elucidation. Indeed, the ${ }^{1} \mathrm{H}$ NMR spectrum of the deuterated mixture of complex 12 (Figure 4.33) was very similar to the ${ }^{1} \mathrm{H}$ NMR spectrum of the non-deuterated complex 12 (Figure 4.12). The ${ }^{31} \mathrm{P}$ NMR spectrum was very similar to the one observed in Figure 4.34. The chemical shift of the ${ }^{31} \mathrm{P}$ NMR signals were slightly shifted as the absence of cryptand likely affected the chemical environment of the ${ }^{31} \mathrm{P}$ nuclei. 


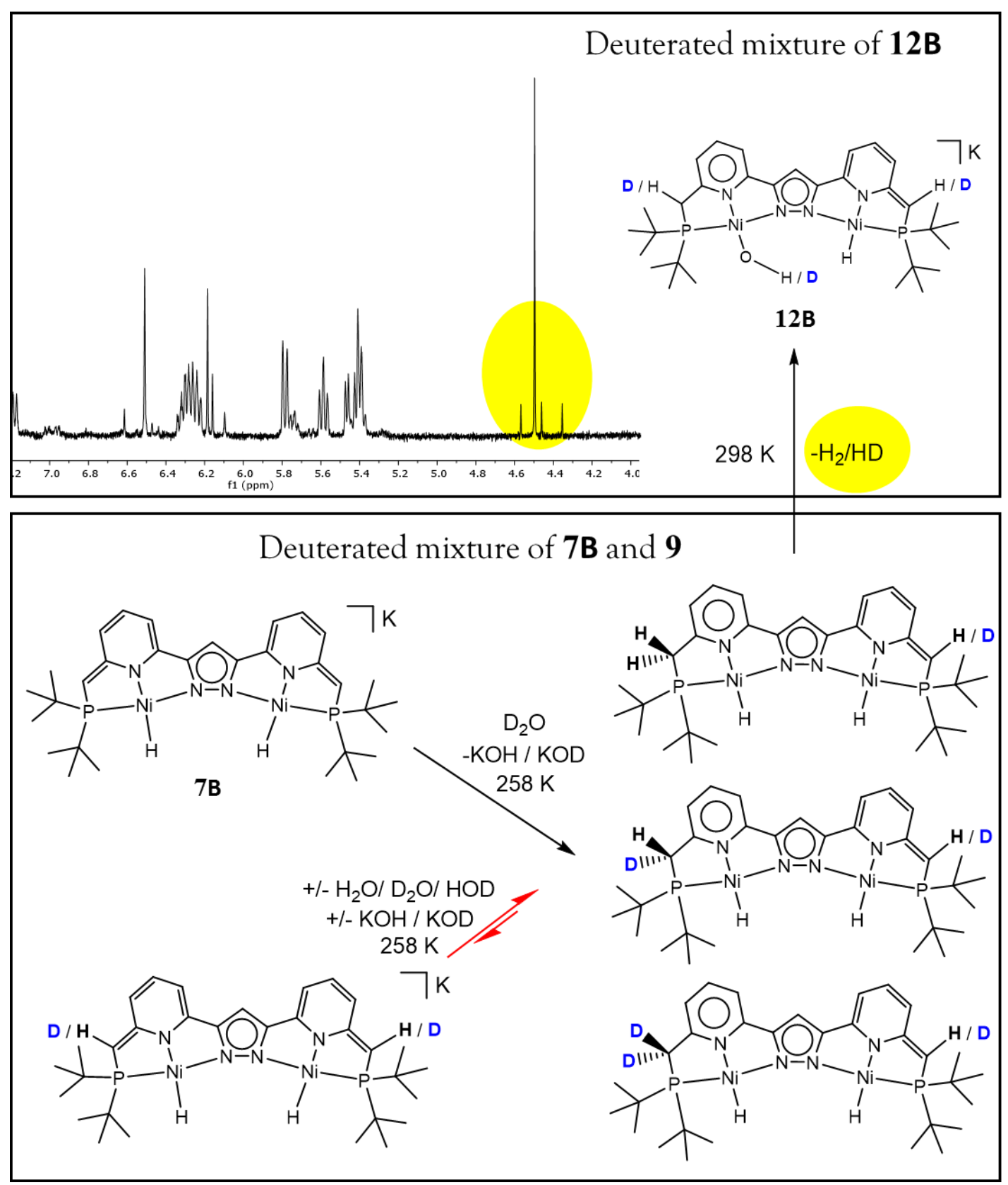

Figure 4.37. ${ }^{1} \mathrm{H}$ NMR spectrum of the deuterated mixture of $\mathbf{7 B}$ and $\mathbf{9}$ en route to the deuterated mixture of $\mathbf{1 2} \mathbf{B}$ in THF-d ${ }^{8}$ at $298 \mathrm{~K}$.

The ${ }^{31} \mathrm{P}$ NMR spectrum depicted in Figure 4.38 was analysed more in details. The hydrolysis of complex 7 with $\mathrm{D}_{2} \mathrm{O}$ gave a deuterated mixture of complex 12. Eight signals were observed. If all isotope shifts were considered in the possible combinations of deuterated complex $\mathbf{1 2}$, it was only possible to expect six signals. A list of the possible combinations helped to understand these possibilities: 


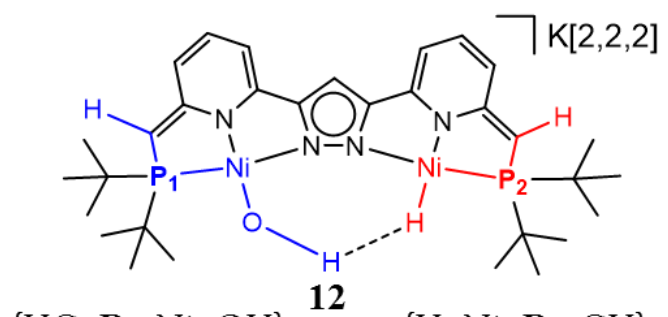

$\left\{\mathrm{HC}-\mathrm{P}_{1}-\mathrm{Ni}-\mathrm{OH}\right\}$

$\left\{\mathrm{H}-\mathrm{Ni}-\mathrm{P}_{2}-\mathrm{CH}\right\}$

All the fragments that could give different ${ }^{31} \mathrm{P}$ NMR signals for the deuterated complex $\mathbf{1 2}$ were the following : $\left\{\mathrm{HC}-\mathrm{P}_{1}-\mathrm{Ni}-\mathrm{OH}\right\},\left\{\mathrm{DC}-\mathrm{P}_{1}-\mathrm{Ni}-\mathrm{OH}\right\},\left\{\mathrm{HC}-\mathrm{P}_{1}-\mathrm{Ni}-\mathrm{OD}\right\},\left\{\mathrm{DC}-\mathrm{P}_{1}-\mathrm{Ni}-\mathrm{OD}\right\}$, $\left\{\mathrm{H}-\mathrm{Ni}-\mathrm{P}_{2}-\mathrm{CH}\right\},\left\{\mathrm{H}-\mathrm{Ni}-\mathrm{P}_{2}-\mathrm{CD}\right\}$. However, the ${ }^{31} \mathrm{P}$ NMR spectra involved 8 signals. The DHB studied in part 4.3 possibly influenced the chemical shift of the ${ }^{31} \mathrm{P}$ NMR signals, so that additional ${ }^{31}$ P NMR signals could be observed. It was unlikely that the deuteration of the hydroxyl unit on the $\mathrm{P}_{1}$ moiety would have influenced the chemical shift of the phosphorus atom $\mathrm{P}_{2}$ because of their significant interatomic distance. However, the hydroxyl was interacting with the hydride by means of dihydrogen bond. Thus, two possible types of interaction between $\{\mathrm{NiO}-\mathrm{H} \cdots \mathrm{H}-\mathrm{Ni}\}$ and $\{\mathrm{NiO}-\mathrm{D} \cdots \mathrm{H}-\mathrm{Ni}\}$ were probably influencing the chemical shift of the ${ }^{31} \mathrm{P}$ NMR signals. A representation was given in Figure 4.38.
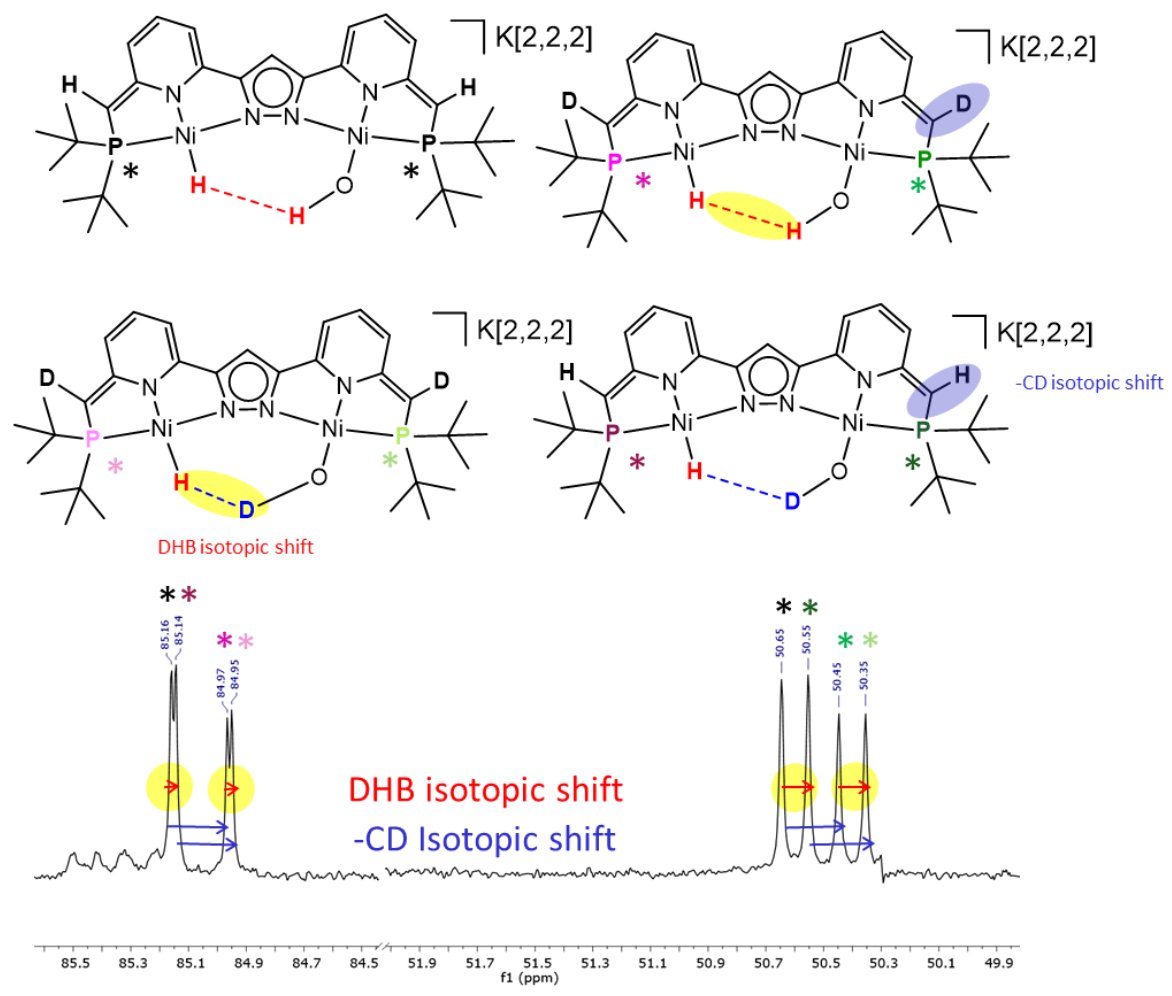

Figure 4.38. ${ }^{31} \mathrm{P}$ NMR spectrum of the mixture of deuterated complex $\mathbf{1 2 B}$ in THF-d ${ }^{8}$ at 298 $\mathrm{K}$. The spectrum was truncated for more clarity. 


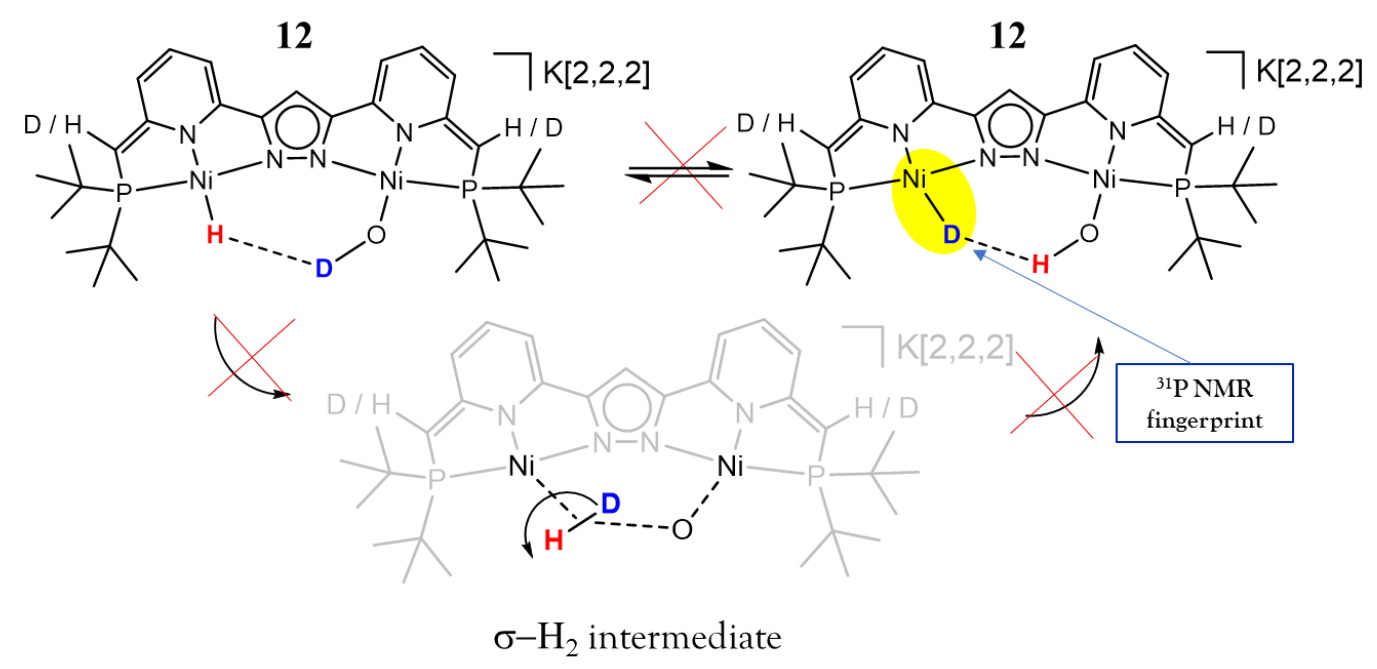

Figure 4.39. Mechanism for plausible intramolecular H/D exchange mediated by the DHB within complex 12.

Despite the complexity of the mixture of deuterated complex $\mathbf{1 2}$ observed by ${ }^{31} \mathrm{P}$ NMR spectroscopy in Figure 4.36, an important common feature was observed between the complexes of this mixture. In the previous experiment, the addition of $\mathrm{D}_{2} \mathrm{O}$ to complex $\mathbf{7}$ did not influenced the ${ }^{1} \mathrm{H}$ NMR Ni-H signals at $258 \mathrm{~K}$ (Figure 4.33). Thus, the $\mathrm{Ni}-\mathrm{H}$ moiety subsisted in presence of $\mathrm{H}_{2} \mathrm{O}$ or $\mathrm{D}_{2} \mathrm{O}$ and no formation of $\mathrm{Ni}-\mathrm{D}$ moiety was observed. However, some $\mathrm{NiO}-\mathrm{D}$ moiety formed when $\mathrm{D}_{2} \mathrm{O}$ was used for the hydrolysis. It was interesting to ask if an $\mathrm{H} / \mathrm{D}$ exchange between $\mathrm{Ni}-\mathrm{H}$ and $\mathrm{Ni}-\mathrm{OD}$ mediated by the DHB according to the mechanism depicted in Figure 4.39 would occur. This question was answered by the analysis of the ${ }^{31} \mathrm{P}$ NMR spectrum represented in Figure 4.38. Even after a long exposition time of the NMR sample, no change in the multiplicity of the ${ }^{31} \mathrm{P}$ NMR signals were observed. The formation of $(1: 1: 1)^{31} \mathrm{P}$ NMR triplet typical for Ni-D moiety were not observed. Thus, it was likely that the H/D exchange represented in Figure 4.39 was not occurring.

\subsection{Isolation of a side product}

Even if the synthesis of complex $\mathbf{1 2}$ appeared straightforward through addition of water to complex 7, the reaction was sometimes hampered by the presence of traces of silicon grease in the deuterated solvent. Complex 12 reacted easily with grease as it was observed in Figure 4.32 (the formation of the side product was notified by: $\mathbf{\Delta}$ ). However, grease did not react with complex $\mathbf{7}$ alone. Complex $\mathbf{1 1}$ started to form only when reasonable amounts of compound $\mathbf{1 2}$ formed. Complex 11 could be isolated as $\mathrm{K}[2,2,2]\left[\mathrm{LNi}\left(\mu-\mathrm{O}_{2} \mathrm{SiMe}_{2}\right) \mathrm{Ni}\right](\mathbf{1 1})$ and was fully characterized by NMR methods. Interestingly, the X-ray structure revealed a doubly dearomatized backbone, akin to complexes $\mathbf{7 , 8}$ and $\mathbf{1 2}$. 

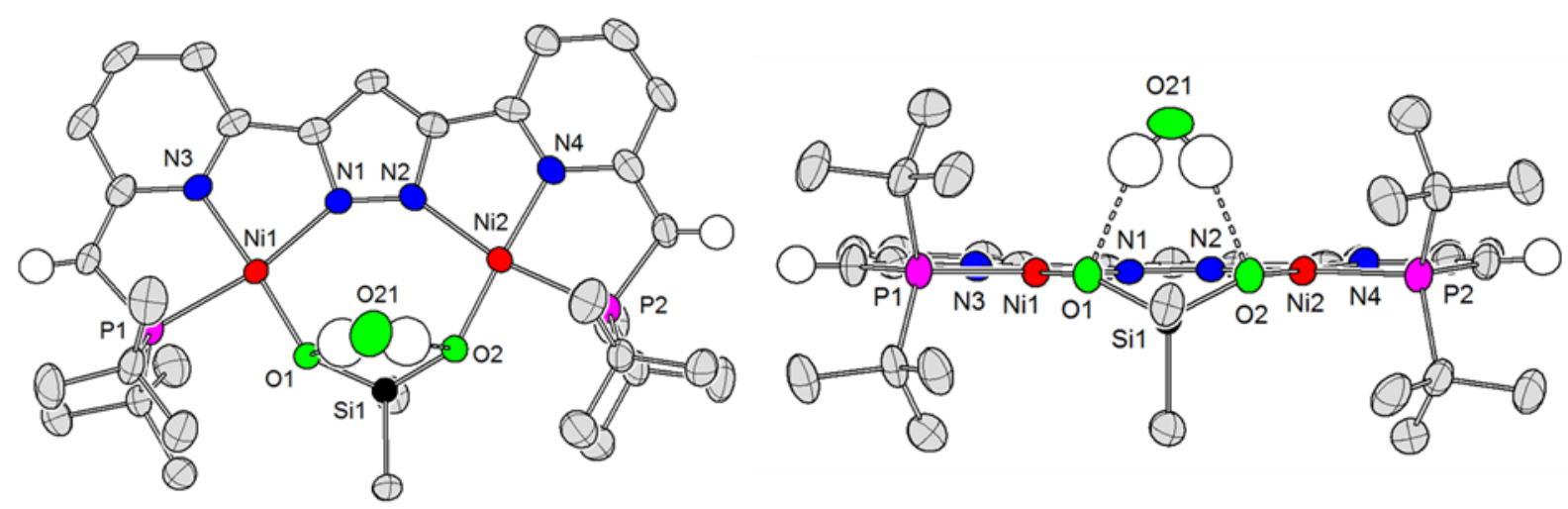

Figure 4.40 Molecular structure (thermal displacement ellipsoids shown at $50 \%$ probability) of the anion of complex 11. Most of the hydrogen atoms and all cations have been omitted for clarity. Left: top view of the molecular structure. Right: front view of the molecular structure

\begin{tabular}{|c|c|c|c|}
\hline Bond Length & $\mathrm{nd} \mathrm{Ni(1)/ \AA}$ & \multicolumn{2}{|c|}{ Bond Lengths around $\mathrm{Ni}(2) / \AA$} \\
\hline $\mathrm{Ni}(1)-\mathrm{O}(1)$ & $1.833(2)$ & $\mathrm{Ni}(2)-\mathrm{O}(2)$ & $1.834(2)$ \\
\hline $\mathrm{Ni}(1)-\mathrm{N}(3)$ & 1.884(3) & $\mathrm{Ni}(2)-\mathrm{N}(4)$ & $1.876(3)$ \\
\hline $\mathrm{Ni}(1)-\mathrm{N}(1)$ & $1.972(3)$ & $\mathrm{Ni}(2)-\mathrm{N}(2)$ & $1.972(3)$ \\
\hline $\mathrm{Ni}(1)-\mathrm{P}(1)$ & $2.1717(10)$ & $\mathrm{Ni}(2)-\mathrm{P}(2)$ & $2.1728(10)$ \\
\hline $\mathrm{Si}(1)-\mathrm{O}(1)$ & $1.609(2)$ & & \\
\hline $\mathrm{Si}(1)-\mathrm{O}(2)$ & $1.610(3)$ & & \\
\hline $\mathrm{Si}(1)-\mathrm{C}(32)$ & $1.871(4)$ & & \\
\hline $\mathrm{Si}(1)-\mathrm{C}(33)$ & $1.872(4)$ & & \\
\hline
\end{tabular}

Table 4.2 Selected bond lengths 


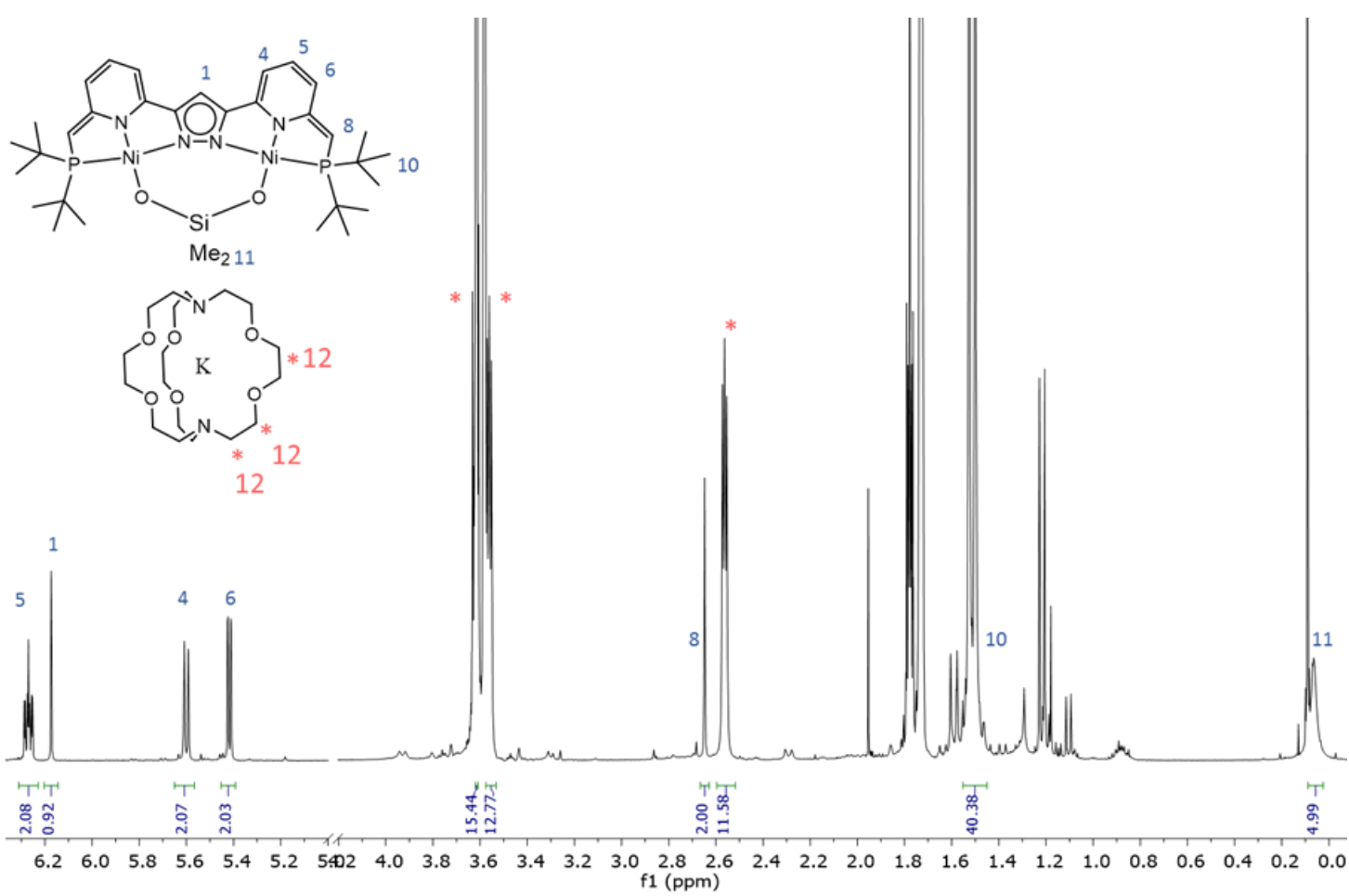

Figure 4.41. ${ }^{1} \mathrm{H}$ NMR of complex 11 in THF-d ${ }^{8}$.

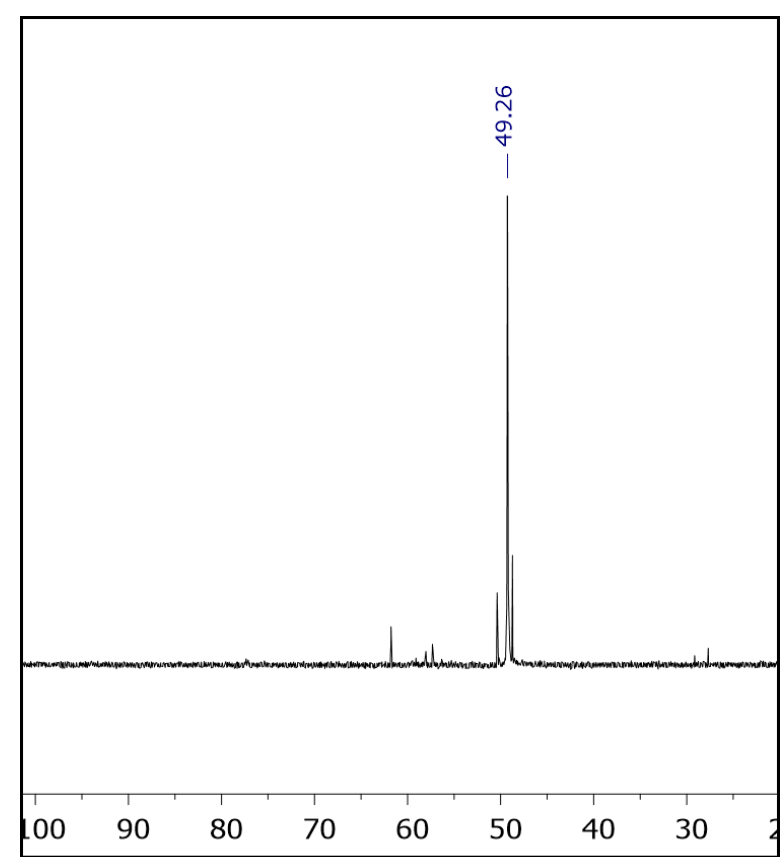

Figure 4.42. ${ }^{31} \mathrm{P}$ NMR of complex 11 in THF-d ${ }^{8}$. The chemical shift of this complex can be observed during the hydrolysis reaction. Example within Figure $4.30(\boldsymbol{\Delta})$. 


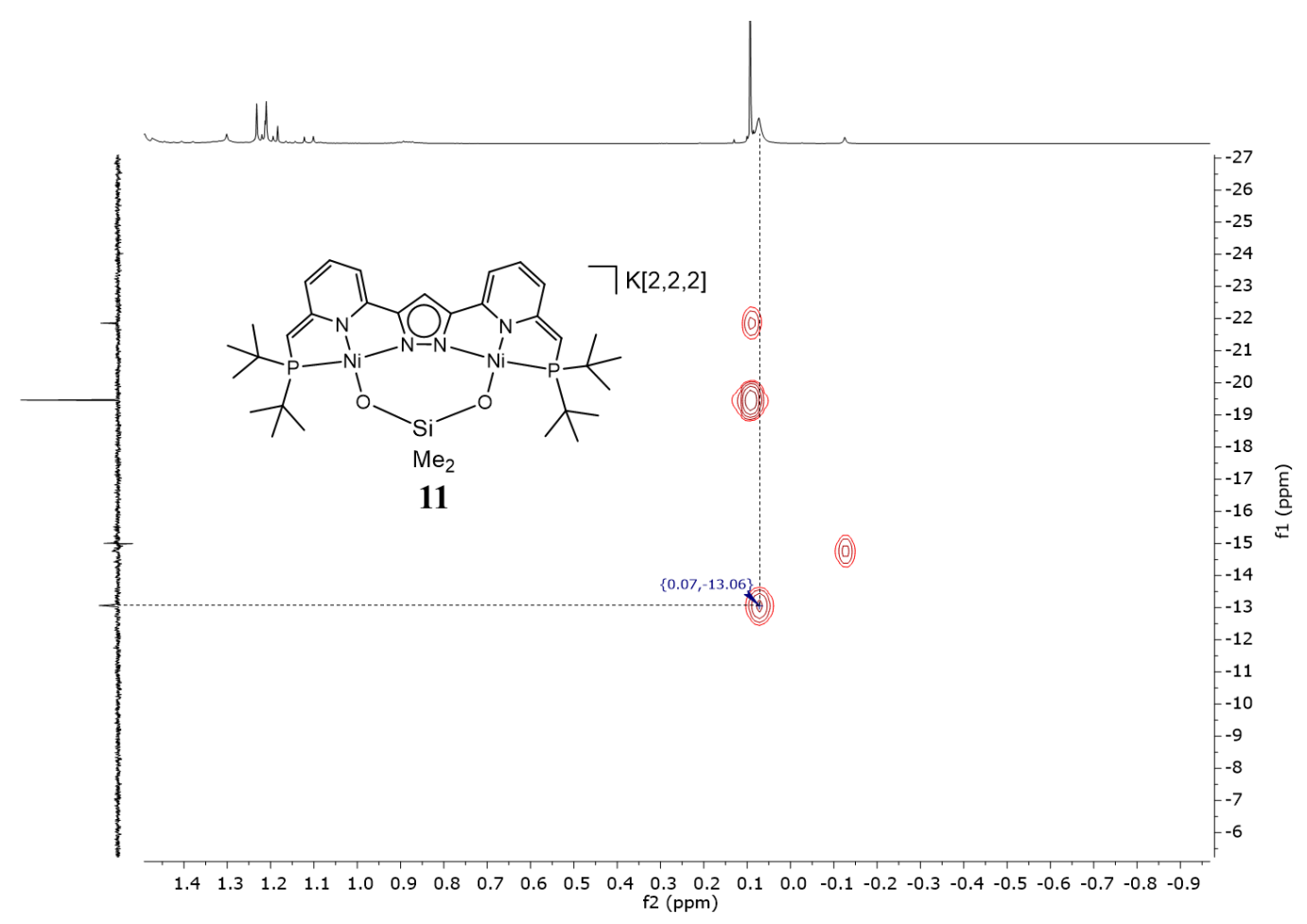

Figure 4.43. ${ }^{29} \mathrm{Si} /{ }^{1} \mathrm{H}$ HMBC of complex 11 in $\mathrm{THF}-\mathrm{d}^{8}$ at $328 \mathrm{~K}$.

\subsection{Protonation with $\mathrm{HBAr}_{4}^{\mathrm{F}}$}

Since the protonation of the side arm was known to occur under weakly acidic conditions with $\mathrm{H}_{2} \mathrm{O}$, an experiment was conducted were the acid was stronger. The addition of a strong acid to complex 7 was monitored by NMR spectroscopy. It was observed that $\mathrm{HBAr}_{4}{ }_{4}$ tended to polymerize THF. The small amounts of $\mathrm{HBAr}_{4}{ }_{4}$ reacted with the complex, as indicated by a colour change of the solution. After the addition of $\operatorname{HBAr}_{4}{ }_{4}$, the colour of the solution turned immediately from bordeaux to dark brown. The ${ }^{31} \mathrm{P}$ and ${ }^{1} \mathrm{H}$ NMR spectra obtained were represented in Figure 4.44. Small amounts of $\mathrm{H}_{2}$ were detected as a ${ }^{1} \mathrm{H}$ NMR signal at $4.5 \mathrm{ppm}$. It was possible to identify an upfield ${ }^{1} \mathrm{H}$ NMR signal at $-20.6 \mathrm{ppm}$ which likely indicated two equivalent hydride signals. The ${ }^{1} \mathrm{H}$ NMR signals in the aromatic region were overlapping with those of the $\mathrm{BAr}_{4}{ }_{4}$ ion so that their detection was difficult. A doublet at $1.4 \mathrm{ppm}$ for the $\mathrm{tBu}$ signal was observed. Since the $\mathrm{tBu}$ groups have strong intensities, it was generally a good indicator to estimate if other species formed during the reaction. However, in that case, the region was clear from other signals. A major ${ }^{31} \mathrm{P}$ NMR signal has been observed at $94 \mathrm{ppm}$ and confirmed the preponderance of a symmetric complex in solution. This data together suggested a clean conversion of complex $\mathbf{7}$ to the hypothetical complex 10. Complex $\mathbf{1 0}$ could not be isolated or further characterized. This experiment emphasized the stability of the $\mathrm{Ni}-\mathrm{H}$ moiety in acidic media, which was not the case in presence of water. 


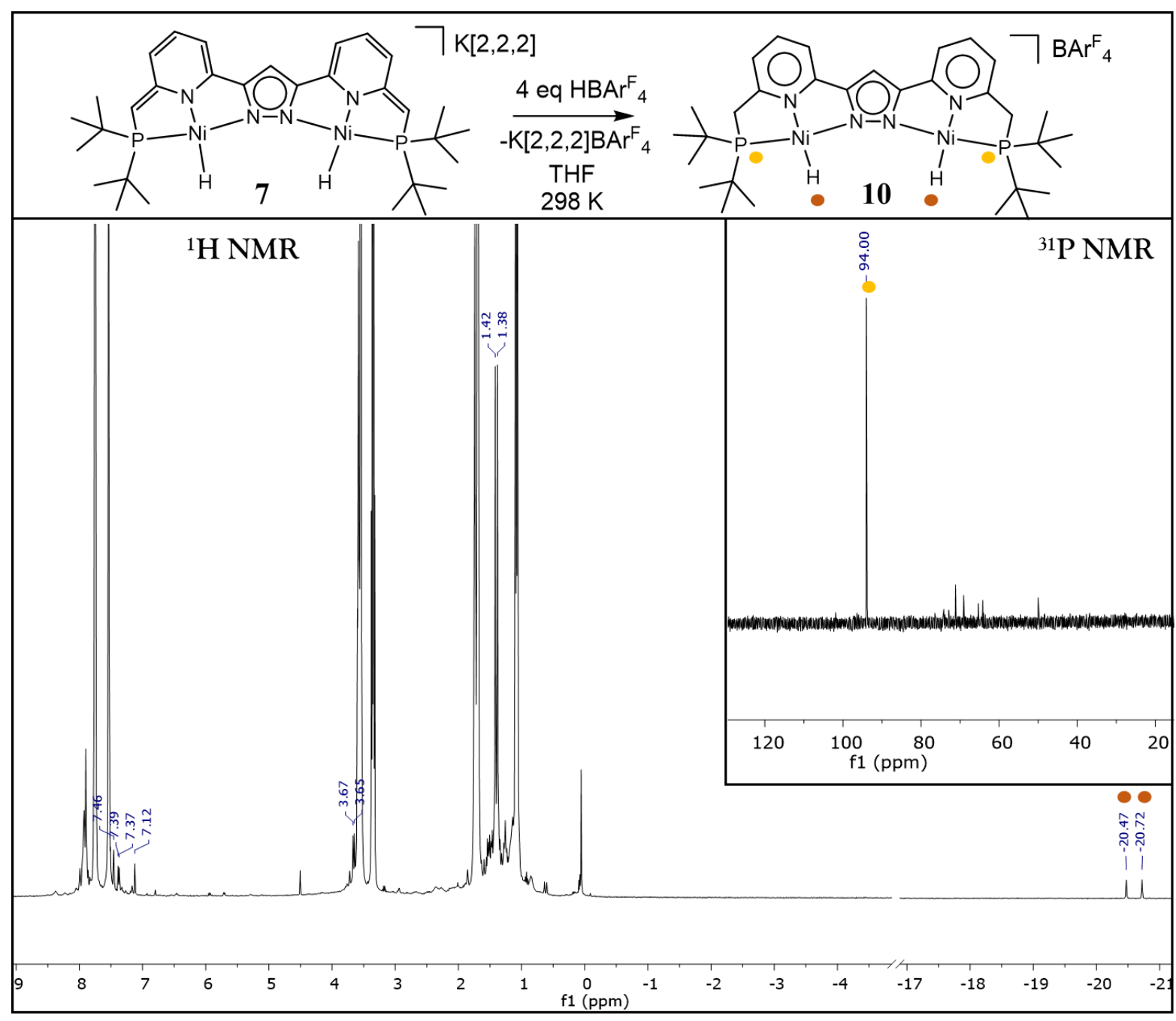

Figure 4.44. ${ }^{1} \mathrm{H}$ and ${ }^{31} \mathrm{P}$ NMR (on the right) spectra of complex $\mathbf{1 0}$ in THF-d ${ }^{8}$ at $328 \mathrm{~K}$.

\subsection{Splitting of $\mathrm{H}_{2} / \mathrm{D}_{2}$}

The reaction of complex $\mathbf{7}$ with water gave complex $\mathbf{1 2}$ and the formation of intermediate $\mathbf{9}$ was proposed. It was not possible to determine how $\mathrm{H}_{2}$ formed and how the intermediate $\mathbf{9}$ further evolved. A proposed mechanism was depicted in Figure 4.29. In the following part, the addition of hydrogen to complex $\mathbf{1 2}$ was performed in order to test if the back reaction of complex $\mathbf{1 2}$ to complex 7 was possible.

Complex 12 was dissolved in THF- $\mathrm{d}^{8}$ and the resulting solution was transferred to a Young NMR tube. The Young tube was connected to the apparatus described in the experimental section. Then dry $\mathrm{H}_{2}$ was added to the sample. The addition of hydrogen in the NMR tube was immediately followed by a colour change from deep violet to deep brown. This colour was reminiscent from the colour of the intermediate $\mathbf{9}$. The colour changes likely indicated that a reaction occurred between complex $\mathbf{1 2}$ and hydrogen. 
The monitoring of such reaction was performed by NMR spectroscopy. In this regard, two important types of experiments have been carried out. The first experiment consisted in the addition of $\mathrm{H}_{2}$ to a deuterated mixture of complex $\mathbf{1 2}$ (the mixture of deuterated complexes was represented in Figure 4.38). A second type of experiment consisted in adding $\mathrm{D}_{2}$ to the nondeuterated complex 12. Both experiments were monitored by ${ }^{1} \mathrm{H}$ and ${ }^{31} \mathrm{P}$ NMR spectroscopy.

The monitoring of the reaction of deuterated 12 with $\mathrm{H}_{2}$ by ${ }^{1} \mathrm{H}$ NMR spectroscopy was represented in Figure 4.45. The ${ }^{1} \mathrm{H}$ NMR signals of complex 12 (the ${ }^{1} \mathrm{H}$ NMR signal of the $\mathrm{Ni}-\mathrm{OH}$ at $-1.8 \mathrm{ppm}$ in particular) slowly decreased over time and gave rise to new signals. After 16 hours, the upfield ${ }^{1} \mathrm{H}$ NMR signal of the hydride moiety of complex $\mathbf{1 2}$ was shifted downfield. A symmetric compound has formed over time as less ${ }^{1} \mathrm{H}$ NMR signals were observed in the region o. An intermediate involving two hydride signals of equal intensities formed after 3 hours. It reached a maximum concentration after 9 hours according to the intensity of the signals. The formation of HD has been observed during the reaction. 


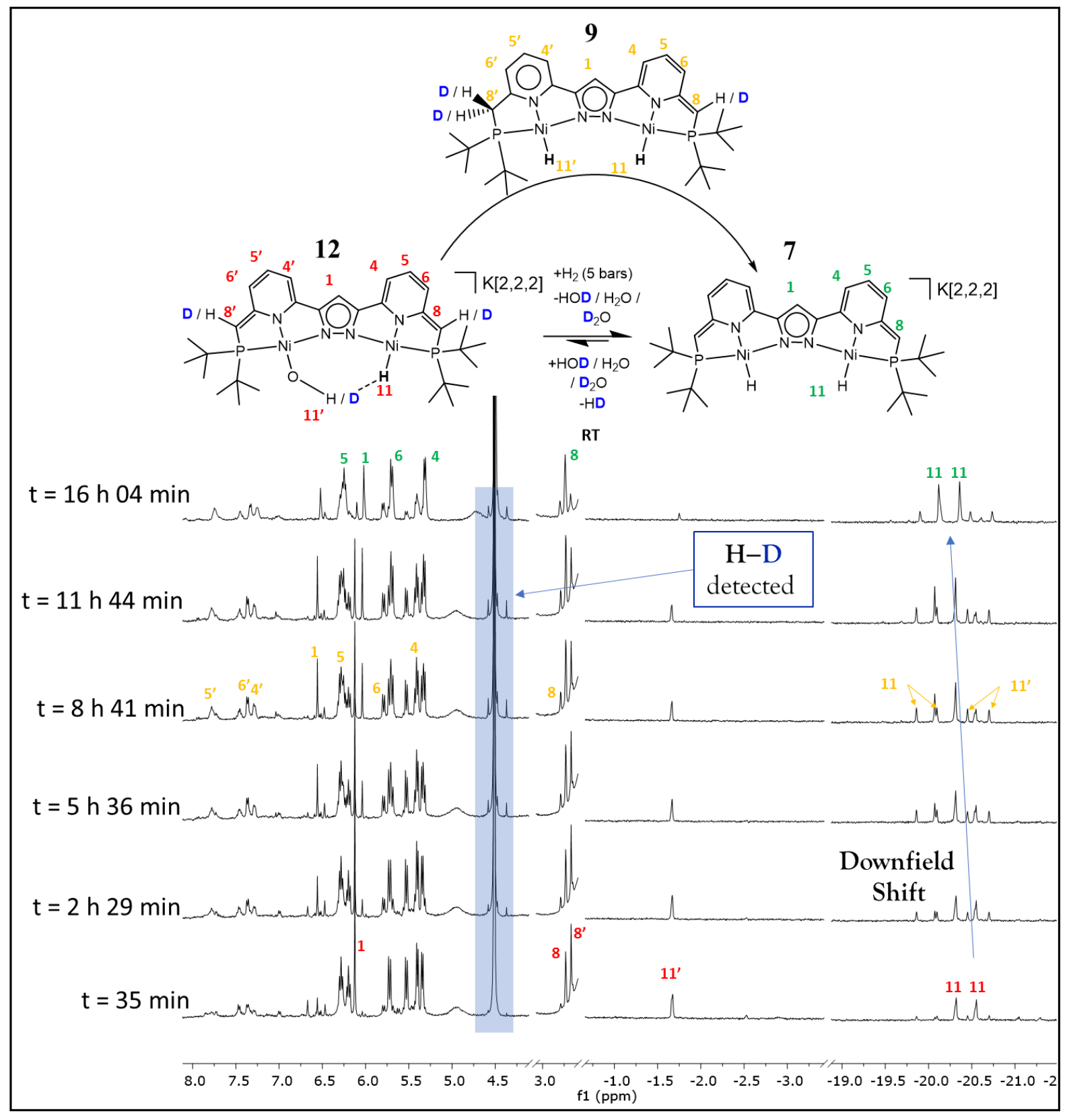

Figure 4.45. Monitoring the reaction of a mixture of deuterated complex 12 with $\mathrm{H}_{2}$ by ${ }^{1} \mathrm{H}$ NMR spectroscopy in THF-d ${ }^{8}$ at $298 \mathrm{~K}$.

The ${ }^{31} \mathrm{P}$ NMR of this reaction was shown in Figure 4.46. The mixture of deuterated complex 12 was indicated by the height coloured asterisks. It indicated the eight ${ }^{31} \mathrm{P} N \mathrm{NR}$ signals of the deuterated mixture of complex 12 (cf Figure 4.38 for the attribution of NMR signals). When $\mathrm{H}_{2}$ was added, the height ${ }^{31} \mathrm{P}$ NMR signals decreased proportionally to each other, which likely indicated a similar reactivity of the different deuterated complexes $\mathbf{1 2}$ toward $\mathrm{H}_{2}$.

The addition of $\mathrm{H}_{2}$ gave rise to new ${ }^{31} \mathrm{P}$ NMR signals. Three downfield ${ }^{31} \mathrm{P}$ NMR signals at 93.15 ppm, $92.89 \mathrm{ppm}$ and $92.62 \mathrm{ppm}$ were spaced by $0.26 \mathrm{ppm}(43 \mathrm{~Hz})$. Those signals proportionally increased with two upfield ${ }^{31} \mathrm{P}$ NMR signals at $83.86 \mathrm{ppm}$ and $83.65 \mathrm{ppm}$, both spaced by 0.21 ppm $(34 \mathrm{~Hz})$. Although the chemical shifts were different in the intermediate 9 of Figure 4.34 (for the three downfield signals respectively: $93.38 \mathrm{ppm}, 93.12 \mathrm{ppm}$ and $92.85 \mathrm{ppm}$ ) the same 
isotope shifts, and the same deuterated mixture was observed in this experiment. The origin of the chemical shift difference observed was likely related to the influence of the cryptand in solution.

Thus, it strongly suggested that complex $\mathbf{9}$ formed after the addition of $\mathrm{H}_{2}$ to complex 12. Two other ${ }^{31} \mathrm{P}$ NMR signals at $84.10 \mathrm{ppm}$ and $83.90 \mathrm{ppm}$ spaced by $0.21 \mathrm{ppm}(34 \mathrm{~Hz})$ were observed and were perfectly in line with the presence of a deuterated population of complex 7 . The data collected from the ${ }^{1} \mathrm{H}$ and ${ }^{31} \mathrm{P}$ NMR spectra and their comparison with the data from part 4.4, 4.5 and 4.6 of this chapter strongly suggested that the reaction of complex $\mathbf{1 2}$ with $\mathrm{H}_{2}$ led to the formation of complex $\mathbf{7}$ and $\mathbf{9}$. An equilibrium in solution between 12, 7 and $\mathbf{9}$ was likely occurring and suggested the reversible splitting of $\mathrm{H}_{2}$.

Because the synthesis of complex $\mathbf{1 2}$ was low yielding and time demanding, a large batch of that complex in order to develop an exhaustive kinetic study could not be prepared. 


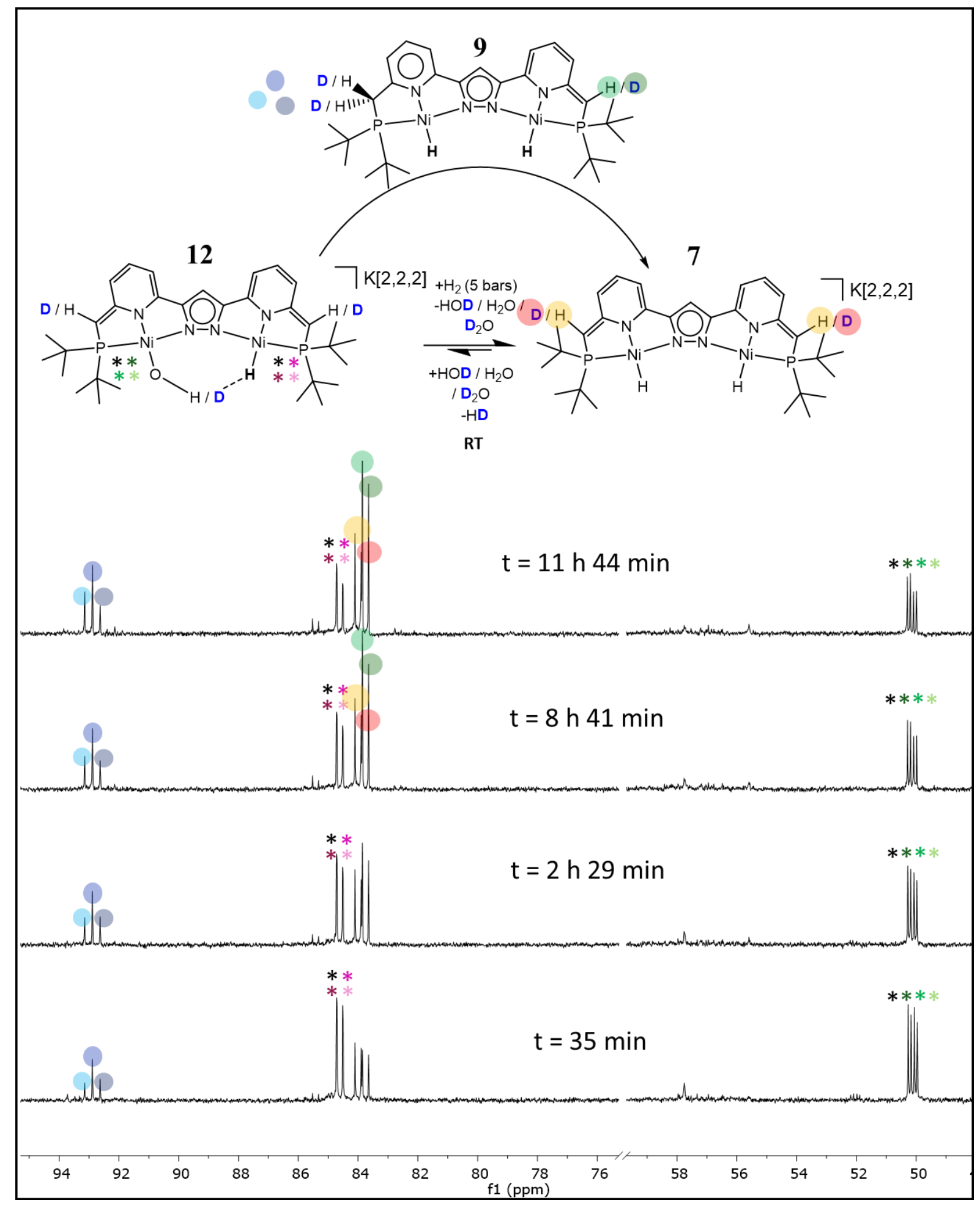

Figure 4.46. Monitoring the reaction of a mixture of deuterated complex 12 with $\mathrm{H}_{2}$ by ${ }^{31} \mathrm{P}$ NMR spectroscopy in THF-d ${ }^{8}$.

A second labelling experiment was performed: the addition of $\mathrm{D}_{2}$ to the non-deuterated complex 12. It was monitored by ${ }^{1} \mathrm{H}$ and ${ }^{31} \mathrm{P}$ NMR spectroscopy at $278 \mathrm{~K}$ and a pressure of 1 bar was used. ${ }^{1} \mathrm{H}$ NMR spectra of this reaction were represented in Figure 4.47. The upfield ${ }^{1} \mathrm{H}$ NMR signals of $\mathrm{Ni}-\mathrm{OH}\left(11^{\prime}\right)$ and $\mathrm{Ni}-\mathrm{H}$ (11) of complex 12 slowly deceased over time. The formation of new 
hydride resonances was observed but their signal intensities were very low. After 30 hours of reaction, almost no signal was detected in the upfield region. The formation of $\mathrm{HD}$ was observed at $4.5 \mathrm{ppm}$. It was interesting to observe that no $\mathrm{H}_{2}$ was detected. The ${ }^{1} \mathrm{H}$ NMR signals for the $\mathrm{CH}$ aliphatic groups almost disappeared. Thus, many ${ }^{1} \mathrm{H}$ NMR signals disappeared during the reaction with $\mathrm{D}_{2}$. However, the ${ }^{1} \mathrm{H}$ NMR signals in the aromatic region were very similar to those found for complex $\mathbf{9}$ and $\mathbf{7}$.

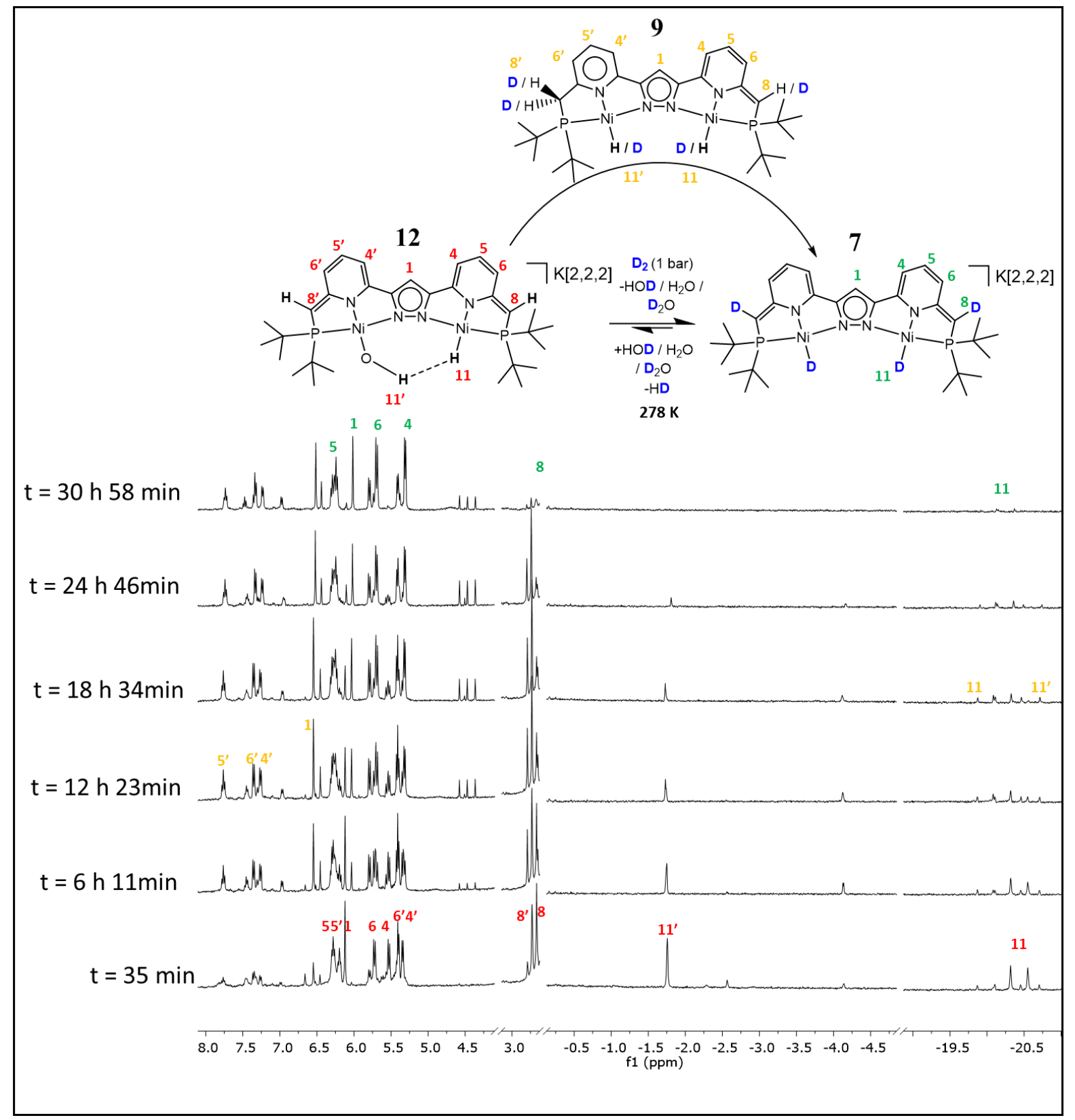

Figure 4.47. Monitoring the reaction of non-deuterated complex 12 with $\mathrm{D}_{2}$ by ${ }^{1} \mathrm{H}$ NMR spectroscopy in THF-d ${ }^{8}$ at $278 \mathrm{~K}$. 


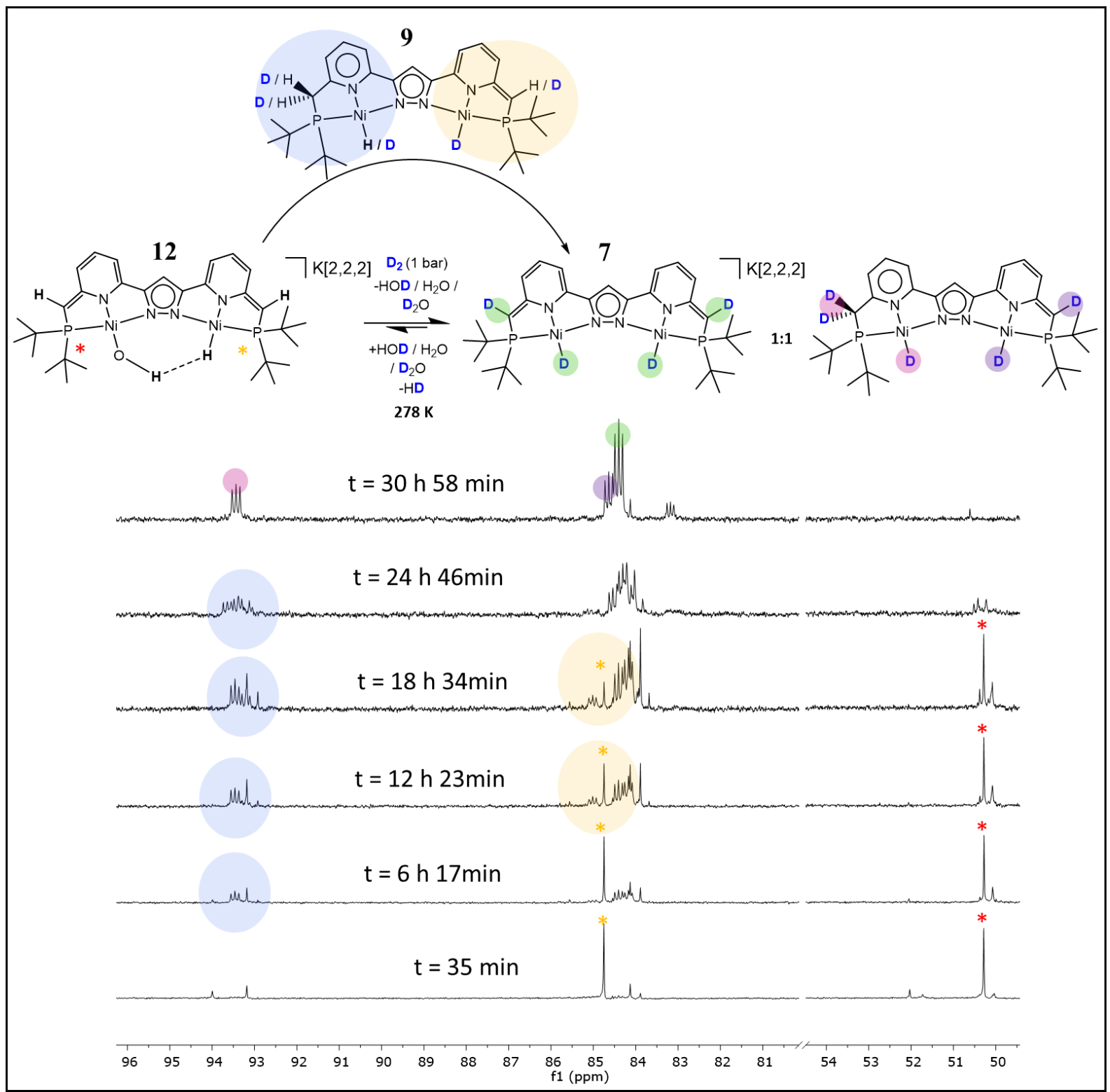

Figure 4.48. Monitoring the reaction of complex 12 with $\mathrm{D}_{2}$ by ${ }^{31} \mathrm{P}$ NMR spectroscopy in THF-d at $278 \mathrm{~K}$.

The ${ }^{31} \mathrm{P}$ NMR spectra of this reaction were presented in Figure 4.48. The presence of complex $\mathbf{1 2}$ was indicated by red and yellow asterisks over the corresponding ${ }^{31} \mathrm{P}$ NMR signals. Those ${ }^{31} \mathrm{P}$ NMR signals decreased over time. New ${ }^{31} \mathrm{P}$ NMR signals formed. Among these, it was possible to observe 1:1:1 multiplets likely indicating the formation of different $\mathrm{Ni}-\mathrm{D}$ moieties. It was in line with the disappearance of the $\mathrm{Ni}-\mathrm{H}$ signals seen in the ${ }^{1} \mathrm{H}$ NMR spectra in Figure 4.47. It was difficult to clearly identify the different complexes forming over time. However, the chemical shift of the new ${ }^{31} \mathrm{P}$ NMR signals were in the same region as the ${ }^{31} \mathrm{P}$ NMR signals of complexes 7 and 9. Thus, the collected data from the ${ }^{1} \mathrm{H}$ and ${ }^{31} \mathrm{P}$ NMR spectra suggested the formation of complex $\mathbf{9}$ and $\mathbf{7}$ when complex $\mathbf{1 2}$ was reacted with $\mathrm{D}_{2}$. 
A comparison of the ${ }^{31} \mathrm{P}$ NMR spectra depicted in Figure 4.46 and Figure 4.48 led to the observation that the multiplicity of the ${ }^{31} \mathrm{P}$ NMR signals were very different from one experiment in respect to the other. In the experiment with $\mathrm{H}_{2}$, NMR signals of the complex $\mathbf{9}$ and $\mathbf{7}$ rather implicated $\mathrm{Ni}-\mathrm{H}$, in line with the singlet observed by ${ }^{31} \mathrm{P}$ NMR spectroscopy (Figure 4.46) and the observation of upfield ${ }^{1} \mathrm{H}$ NMR signals (Figure 4.45). In the experiment with $\mathrm{D}_{2}$, NMR signals of the complex 9 and 7 rather implicated $\mathrm{Ni}-\mathrm{D}$, in line with the 1:1:1 triplet observed by ${ }^{31} \mathrm{P}$ NMR spectroscopy in Figure 4.48 and the absence of upfield ${ }^{1} \mathrm{H}$ NMR signals (Figure 4.47).

Those results suggested that the splitting of hydrogen was performed by the dinickel(II) complex 12. Indeed, when $\mathrm{H}_{2}$ was added to complex 12, Ni-H moieties formed. When $\mathrm{D}_{2}$ was added, $\mathrm{Ni}-\mathrm{D}$ moieties were observed. Thus, it was suggested that one hydrogen of $\mathrm{H}_{2}$ was transferred to the metal as a hydride ligand $\left(\mathrm{H}^{-}\right)$. The experimental data could not clearly indicate where the second hydrogen of $\mathrm{H}_{2}$ was transferred. DFT calculations are currently being performed (in the group of professor Mata) to evaluate the possibility that the $\mathrm{H}^{+}$is ending up at the deprotonated side arm.

\subsection{Discussion}

A discussion was necessary in order to highlight and summarize some features presented along this chapter. It was noteworthy, that the DHB presented in complex $\mathbf{1 2}$ was characterized in THF. The polarity of this solvent is known to disrupt this type of interaction. ${ }^{186}$ So far, DHB were usually characterized in dichloromethane. ${ }^{181} \mathrm{The}\left\{\mathrm{Ni}-\mathrm{H}^{\delta-} \ldots{ }^{\delta+} \mathrm{HO}-\mathrm{Ni}\right\}$ motif in complex $\mathbf{1 2}$ was not sterically constrained and space was available for the rotation of the $\mathrm{OH}$ unit around to the $\mathrm{Ni}-\mathrm{O}$ bond. However, the hydroxyl unit was oriented in a very specific position. The most stable conformation resulted when the hydrogen of the hydroxyl was pointing toward the hydride ligand, ${ }^{213}$ as evidenced by both experiment and DFT.

It was surprising that complex $\mathbf{1 2}$ was stable at $298 \mathrm{~K}$. DHB adducts are known to be difficult to isolate (cf part 4.1). In the dinickel complex synthesized by Manz, an asymmetric hydroxo hydrido complex was observed. However, it decomposed by elimination of $\mathrm{H}_{2}$. The DHB which was evidenced in complex 12 displayed short interatomic $\mathrm{H} \cdots \mathrm{H}$ distances $(<2.4 \AA)$. COSY and NOESY experiment demonstrated the attractive interaction of the two hydrogen atoms. Thus, the collected spectroscopic data strongly evidenced the DHB. A labelling experiment with $\mathrm{D}_{2} \mathrm{O}$ was monitored by ${ }^{31} \mathrm{P}$ NMR spectroscopy and it was suggested that the $H / D$ exchange in $\left\{\mathrm{Ni}-\mathrm{H}^{\delta-} \ldots{ }^{\delta+} \mathrm{DO}-\mathrm{Ni}\right\}$ was unlikely. Thus, it appeared that the combination of the two hydrogen atoms was not favourable. It was probably correlated to the stability of the complex $\mathbf{1 2}$ at $298 \mathrm{~K}$. 

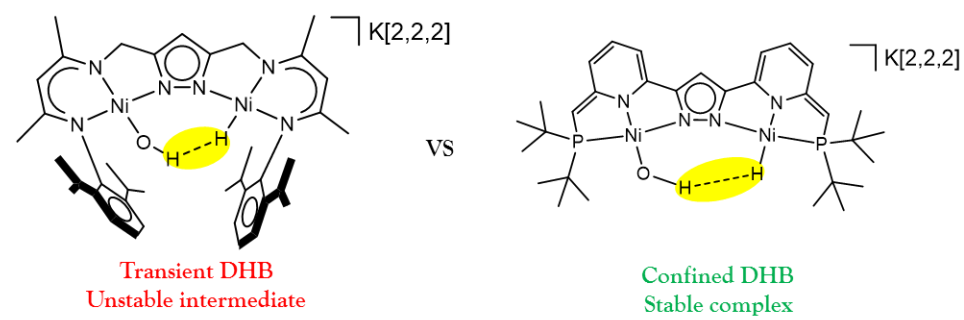

Figure 4.49. Two different pyrazolate based dinickel complexes developed in the Meyer group which possibly featured a DHB.

The mechanism for the formation of complex 7 was investigated. In presence of an excess of $\mathrm{HBAr}_{4}^{\mathrm{F}}$, the formation of complex $\mathbf{1 0}$ was observed. The $\mathrm{Ni}-\mathrm{H}$ moiety within complex $\mathbf{1 0}$ was stable in acidic media. It was assumed that complex $\mathbf{9}$ would quantitatively form and be stable if complex 7 was reacted with a stoichiometric amount of $\operatorname{HBAr}_{4}{ }_{4}$. If water was added to complex $7 \mathbf{B}$ at $258 \mathrm{~K}$, complex 9 formed. However, it was unstable at room temperature and spontaneously eliminate $\mathrm{H}_{2}$, whereby complex $\mathbf{1 2}$ formed. It suggested that the $\mathrm{HO}^{-}$unit had a dramatic influence on complex 9 within the subsequent elimination of $\mathrm{H}_{2}$. The role of $\mathrm{HO}^{-}$could not be spectroscopically evidenced and no other intermediates were clearly identified. DFT calculations to unravel details of this scenario are still ongoing (work in the group of professor Mata).

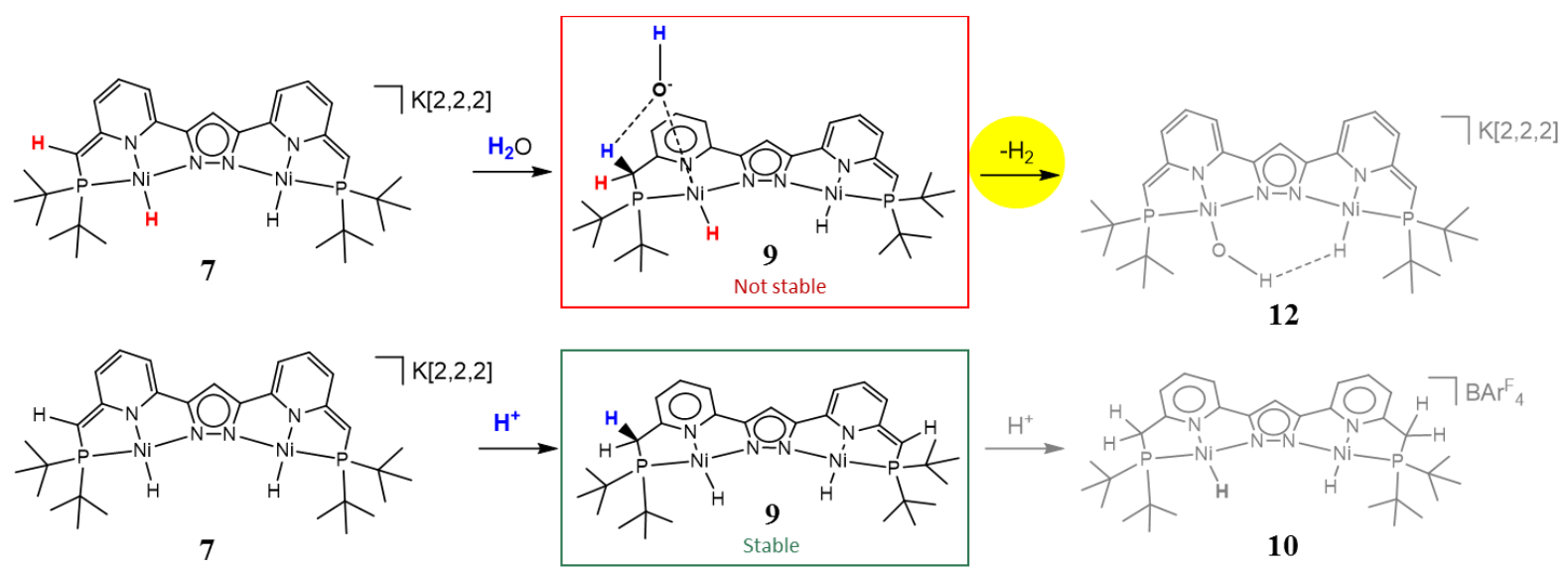

Figure 4.50. Mechanistic proposal for the reactivity of complex 7 in presence of $\mathrm{H} 2 \mathrm{O}$ (top) or with $\operatorname{HBAr}_{4}{ }_{4}$ (bottom).

\subsection{Conclusion}

The present chapter presented the hydrolysis of the dihydride complex $\mathbf{7}$ with water. The reaction gave an asymmetric hydroxido-hydrido complex $\mathbf{1 2}$ which involved a confined dihydrogen bond. The complex was stable at room temperature and differed by its stability from the related intermediate observed by Manz. A mechanistic investigation for the reaction of complex $\mathbf{7}$ to complex 12 was performed. An equilibrium was taking place between water and the deprotonated 
side arm. It was highlighted by labelling experiment with deuterated water. The first step of the hydrolysis could be identified at $248 \mathrm{~K}$. The deprotonation of water was followed by the protonation and the aromatization of one side arm in complex 7B. It gave rise to complex $\mathbf{9}$. Complex 9 spontaneously eliminated $\mathrm{H}_{2}$ at room temperature with the (re)dearomatization of the pyridine. It was not possible to identify further intermediates. Complex $\mathbf{1 2}$ was found to split $\mathrm{H}_{2} / \mathrm{D}_{2}$ by a possible cooperative effect of the ligand. Addition of $\mathrm{H}_{2}$ to this complex led back to the symmetric dihydride complex 7. Thus, a system where complex $\mathbf{7}$ and $\mathbf{1 2}$ are reversibly interconverted respectively by addition of $\mathrm{H}_{2} \mathrm{O}$ and $\mathrm{H}_{2}$ has been established. 


\section{Experimental Section}

\section{General Considerations}

All manipulations were performed under an anaerobic and anhydrous atmosphere of dry argon by using standard Schlenk techniques or in a glove box filled with nitrogen $\left(\mathrm{O}_{2}<0.5 \mathrm{ppm}, \mathrm{H}_{2} \mathrm{O}\right.$ $<0.5 \mathrm{ppm}$ ). Chemicals used were either present in the working group or were purchased from commercial sources, or their synthesis is described below. Glassware was dried at $120^{\circ} \mathrm{C}$ prior to use. THF, hexane were dried over sodium in the presence of benzophenone and were distilled prior to use. DCM and toluene were dried over molecular sieves using a Mbraun PLC. Deuterated solvents were purchased from Euriso-top and transferred directly in the glovebox. Storage of the solvent in the presence of $3 \AA$ activated molecular sieves over 3 days afforded the dry solvent. $D_{2(\mathrm{~g})}$ (Sigma Aldrich, 0.416 L, Quality 99.98\%) and $\mathrm{H}_{2(\mathrm{~g})}$ (Messer, 50 L, Quality 5.0) were dried with an adapted high-pressure apparatus as described below.

IR measurements were performed with a Cary 630 FTIR spectrometer with Dial Path Technology with solid samples and analyzed by FTIR MicroLab software.

Elemental analyses were performed by the analytical laboratory of the Institute of Inorganic Chemistry at Georg-August-University using an Elementar Vario EL III instrument.

Crystal data were collected on a STOE IPDS II diffractometer (graphite monochromated Mo-K $\alpha$ radiation, $\lambda=0.71073 \AA$ ) by use of $\omega$ scans at $-140{ }^{\circ} \mathrm{C}$. The structures were solved by direct methods (SHELXS-2013/14/17) and refined on $\mathrm{F}^{2}$ using all reflections with SHELXL2013/14/17. Non-hydrogen atoms were refined anisotropically. Most hydrogen atoms were placed in calculated positions and assigned to an isotropic displacement parameter of $1.2 \mathrm{U}_{\text {eq }}(\mathrm{C})$.

UV-vis spectra were recorded on Varian Cary 60 and Cary 5000 (UV-vis-NIR) spectrometers using quartz cuvettes $(\mathrm{d}=1 \mathrm{~cm})$ and handled with Agilent CaryUV win softwares.

NMR samples were prepared in normal $3 \mathrm{~mL}$ Young tubes equipped with teflon screwcaps under $\mathrm{N}_{2}$ atmosphere. All reactions of hydrogen splitting were monitored with high pressure tubes. NMR spectra were recorded on Bruker Avance 300, 400 or $500 \mathrm{MHz}$ spectrometers equipped with a liquid $\mathrm{N}_{2}$ evaporator. Spectra were recorded at $298 \mathrm{~K}$ if not stated otherwise. Chemical shifts are reported in parts per million relative to residual signal resonances $\left({ }^{1} \mathrm{H}\right)$ of the deuterated solvent reported in the literature. References for ${ }^{1} \mathrm{H}^{13} \mathrm{C}$ and ${ }^{31} \mathrm{P}$ NMR spectra are TMS, TMS and $\mathrm{H}_{3} \mathrm{PO}_{4}$ respectively. ${ }^{31} \mathrm{P}$ NMR spectra are decoupled from ${ }^{1} \mathrm{H}$ using Garp instead of Waltz16 decoupling pulse programm with an irradiation of the proton centred at $-7 \mathrm{ppm}$. Relaxation times are measured by standard inversion recovery sequence with a list of 10 delay times $(0.01 \mathrm{~s}$, $0.1 \mathrm{~s}, 0.3 \mathrm{~s}, 0.6 \mathrm{~s}, 1 \mathrm{~s}, 1.5 \mathrm{~s}, 2 \mathrm{~s}, 3 \mathrm{~s}, 5 \mathrm{~s}, 10 \mathrm{~s})$. All FIDs are processed with an exponential multiplication prior to Fourier transform. Spectra are baseline and phase corrected before they are fitted with the $T_{1} / T_{2}$ relaxation module of the TopSpin (version 3.2). Percent error was $<4 \%$.

Mass Spectra were recorded on a Brucker HCT Ultra. EI spectrograms with a Finnigan MAT 8200 . 


\section{Ligand Synthesis}

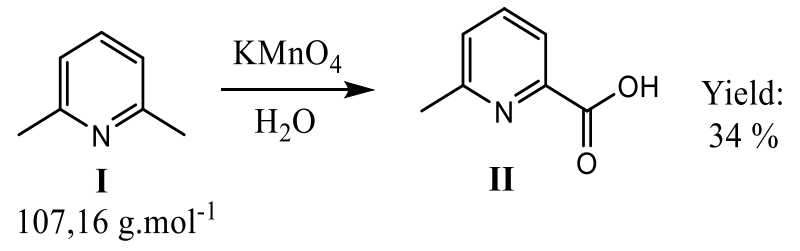

The synthesis was performed according to the literature procedure: ${ }^{214}$ 2,6-lutidine $(114 \mathrm{~g}, 1.06$ mol, 1.0 eq) was dissolved in $4 \mathrm{~L}$ of water and heated to $65^{\circ} \mathrm{C} . \mathrm{KMnO}_{4}(358 \mathrm{~g}, 2.26 \mathrm{~mol}, 2.0 \mathrm{eq})$ was added over 6 hours and the mixture was stirred overnight at $65^{\circ} \mathrm{C}$. The mixture was filtered with a Büchner apparatus and the $\mathrm{MnO}_{2}$ cake was washed with hot water $(1 \mathrm{~L})$. The solution was concentrated to $200 \mathrm{~mL}$ and it was acidified with a $6 \mathrm{M} \mathrm{HCl}$ aqueous solution. When $\mathrm{pH}$ approached 5 a white precipitate started to form, consistent with the protonation of the carboxylate moieties. After further acidification to $\mathrm{pH}=3,3$ toluene $(200 \mathrm{~mL})$ was added and a Dean Stark apparatus allowed to remove of water. $\mathrm{KCl}$ then precipitated. After complete water removal, the flask was disconnected from the apparatus and two portions of $100 \mathrm{~mL}$ boiling toluene were added (use appropriate safety conditions!) which the 6-methyl-2-picolinic acid dissolves. It was filtered with a Büchner apparatus and the $\mathrm{KCl}$ with the dipicolinic acid were removed this way. Cooling the filtrate lead to precipitation of the II and a complete evaporation of the volatile allowed the isolation of a pure white powder $(50 \mathrm{~g}, 0.36 \mathrm{~mol})$.

${ }^{1} \mathrm{H}$ NMR (300 MHz, CDCl $): 10.21(\mathrm{br}, 1 \mathrm{H}) 8.03\left(\mathrm{~d},{ }^{3} \mathrm{~J}=7.62 \mathrm{~Hz}, 1 \mathrm{H}\right) 7.83\left(\mathrm{t},{ }^{3} \mathrm{~J}=7.62 \mathrm{~Hz}, 1 \mathrm{H}\right)$ $7.43\left(\mathrm{~d},{ }^{3} \mathrm{~J}=7.62 \mathrm{~Hz}, 1 \mathrm{H}\right) 2.62(\mathrm{~s}, 3 \mathrm{H})$

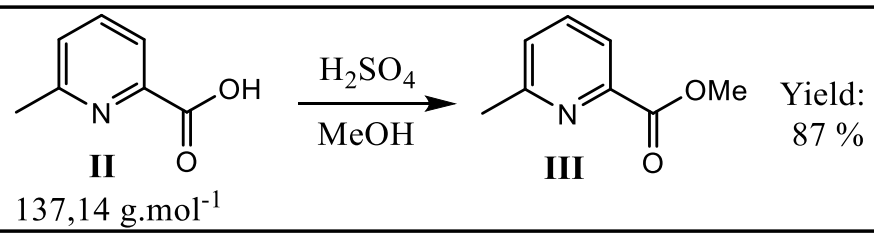

The synthesis was performed according to the literature procedure: ${ }^{50} 6$-methyl-2-picolinic acid (50 $\mathrm{g}, 0.36 \mathrm{~mol}$ ) was dissolved in $300 \mathrm{~mL} \mathrm{MeOH}$ (excess) and a dropwise addition of $50 \mathrm{~mL}$ of $\mathrm{H}_{2} \mathrm{SO}_{4}$ (95-98\%) at room temperature led to formation of a white precipitate that redissolved upon heating. The solution was refluxed 12 hours. The solution was diluted with cold water $(300 \mathrm{~mL})$ and it was neutralized with $\mathrm{K}_{2} \mathrm{CO}_{3}$. Water $(1 \mathrm{~L})$ was added and the mixture was transferred in a separatory funnel. The compound was extracted with three portions of $300 \mathrm{~mL}$ methylene chloride. The fractions were collected and dried over $\mathrm{MgSO}_{4}$. After filtration the solvent was removed to afford III as a pure light-yellow oil (47 g, $0.31 \mathrm{~mol})$.

${ }^{1} \mathrm{H}$ NMR $\left(300 \mathrm{MHz}, \mathrm{CDCl}_{3}\right): 7.94\left(\mathrm{~d},{ }^{3} \mathrm{~J}=7.62 \mathrm{~Hz}, 1 \mathrm{H}\right) 7.71\left(\mathrm{t},{ }^{3} \mathrm{~J}=7.62 \mathrm{~Hz}, 1 \mathrm{H}\right) 7.34\left(\mathrm{~d},{ }^{3} \mathrm{~J}=\right.$ $7.62 \mathrm{~Hz}, 1 \mathrm{H}) 3.99(\mathrm{~s}, 3 \mathrm{H}) 2.65(\mathrm{~s}, 3 \mathrm{H})$ 


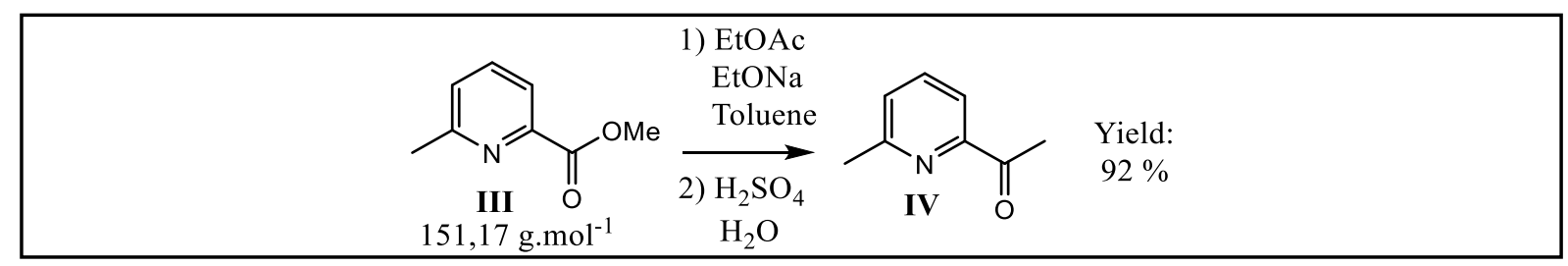

The synthesis was performed according to the literature procedure: ${ }^{50}$ the reaction was carried out under argon atmosphere. $\mathrm{NaOMe}(21.7 \mathrm{~g}, 0.32 \mathrm{~mol})$ was suspended in a mixture of dry EtOAc $(150 \mathrm{~mL})$ and toluene $(75 \mathrm{~mL})$ and the color of the mixture was orange. A dropwise addition of methyl-6-metyl-2-picolinate $(30 \mathrm{~g}, 0.20 \mathrm{~mol}$ ) during $90 \mathrm{~min}$ led to dissolution of $\mathrm{NaOMe}$ with a colour change to red. The solution was refluxed overnight. An orange precipitate formed, and the solution was yellow-orange. The toluene was removed under vacuum and then $68.5 \mathrm{~mL}$ of $\mathrm{H}_{2} \mathrm{SO}_{4}(95-98 \%$ ) diluted in $335 \mathrm{~mL}$ of distilled water was added directly to the orange residue. Gas started to form consistent with the elimination of $\mathrm{CO}_{2}$ from the reaction. The reaction mixture was refluxed for 12 hours. Then it was poured onto ice $(200 \mathrm{~mL})$ and neutralized with $\mathrm{K}_{2} \mathrm{CO}_{3}$. After addition of water $(300 \mathrm{~mL})$, the compound was extracted with three portions of methylene chloride $(200 \mathrm{~mL})$. The organic layers were combined and dried over $\mathrm{MgSO}_{4}$. After filtration, the solvent was removed, and IV was obtained as a brown pure oil ( $24 \mathrm{~g}, 0.18)$.

${ }^{1} \mathrm{H}$ NMR $\left(300 \mathrm{MHz}, \mathrm{CDCl}_{3}\right): 7.83\left(\mathrm{~d},{ }^{3} \mathrm{~J}=7.72 \mathrm{~Hz}, 1 \mathrm{H}\right) 7.69\left(\mathrm{t},{ }^{3} \mathrm{~J}=7.72 \mathrm{~Hz}, 1 \mathrm{H}\right) 7.31\left(\mathrm{~d},{ }^{3} \mathrm{~J}=\right.$ $7.72 \mathrm{~Hz}, 1 \mathrm{H}) 2.71(\mathrm{~s}, 3 \mathrm{H}) 2.61(\mathrm{~s}, 3 \mathrm{H})$

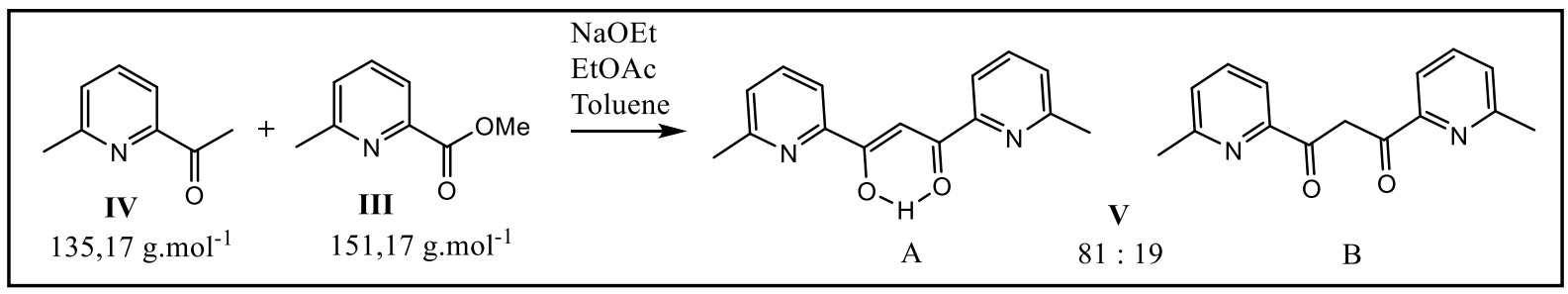

The synthesis was performed according to the literature procedure: ${ }^{50}$ the reaction was carried out under argon atmosphere. $\mathrm{NaOMe}(16 \mathrm{~g}, 0.24 \mathrm{~mol})$ was suspended in $200 \mathrm{~mL}$ dry toluene and the ester III ( $25.8 \mathrm{~g}, 0.17 \mathrm{~mol})$ was added to the suspension via syringe. The solution was orange and a slow dropwise addition at room temperature of the IV (24 g, $0.17 \mathrm{~mol})$ diluted in dry toluene $(200 \mathrm{~mL})$ changed the colour of the solution to dark orange-red. After addition it was stirred for 12 hours at room temperature and then 18 hours at $70^{\circ} \mathrm{C}$. The toluene was then removed under vacuum and $200 \mathrm{~mL}$ of an aqueous $\mathrm{CH}_{3} \mathrm{COOH}$ solution $\left(100 \mathrm{~mL} \mathrm{CH}_{3} \mathrm{COOH}\right.$ (99.5\%) in $100 \mathrm{~mL} \mathrm{H}_{2} \mathrm{O}$ ) was added. A light orange precipitate formed. It was filtered with a Büchner apparatus, washed with portions of water and dried under vacuum overnight. NMR of the $\mathbf{V}$ was recorded before the next reaction step.

${ }^{1} \mathrm{H}$ NMR (300 MHz, CDCl $)$ ): For the enol A: $16.02\left(\mathrm{br}, 1 \mathrm{H}_{\mathrm{A}}\right) 7.95\left(\mathrm{br}, 1 \mathrm{H}_{\mathrm{A}}\right) 7.73\left(\mathrm{~d},{ }^{3} \mathrm{~J}=7.75\right.$ $\left.\mathrm{Hz}, 2 \mathrm{H}_{\mathrm{A}}\right) 7.73\left(\mathrm{t},{ }^{3} \mathrm{~J}=7.75 \mathrm{~Hz}, 2 \mathrm{H}_{\mathrm{A}}\right) 7.29\left(\mathrm{~d},{ }^{3} \mathrm{~J}=7.75 \mathrm{~Hz}, 2 \mathrm{H}_{\mathrm{A}}\right) 2.66\left(\mathrm{~d}, 6 \mathrm{H}_{\mathrm{A}}\right)$. For the diketone B: $7.87\left(\mathrm{~d},{ }^{3} \mathrm{~J}=7.72 \mathrm{~Hz}, 2 \mathrm{H}_{\mathrm{B}}\right) 7.68\left(\mathrm{t},{ }^{3} \mathrm{~J}=7.72 \mathrm{~Hz}, 2 \mathrm{H}_{\mathrm{B}}\right) 7.24\left(\mathrm{~d},{ }^{3} \mathrm{~J}=7.72 \mathrm{~Hz}, 2 \mathrm{H}_{\mathrm{B}}\right) 4.83\left(\mathrm{~s}, 2 \mathrm{H}_{\mathrm{B}}\right)$ $2.40\left(\mathrm{~s}, 6 \mathrm{H}_{\mathrm{B}}\right)$ 


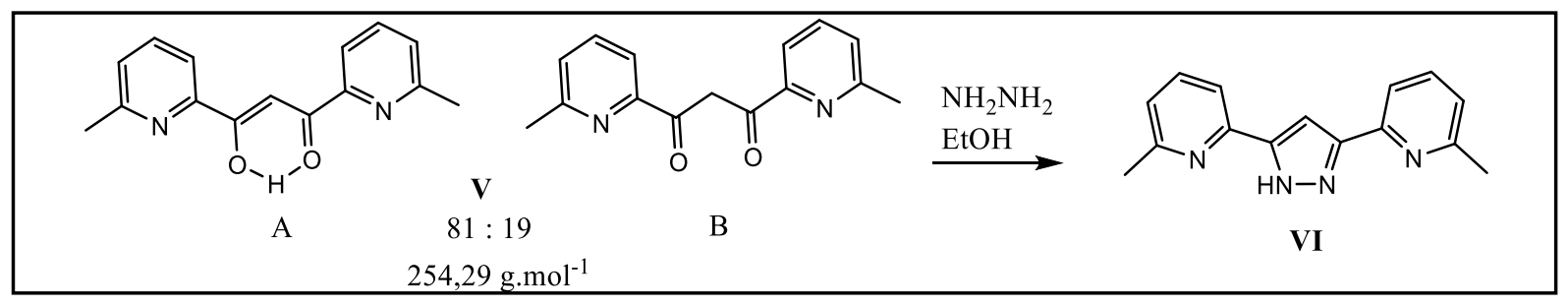

The synthesis was performed according to the literature procedure: ${ }^{50}$ the residue of the previous reaction was suspended in $\mathrm{EtOH}$ and $65 \% \mathrm{NH}_{2} \mathrm{NH}_{2}(25 \mathrm{~mL}, 3 \mathrm{eq})$ was added whereby the solution turned deep red. After $15 \mathrm{~min}$ a precipitate was formed. The mixture was refluxed for 24 hours. The solvent was removed under vacuum and the residue was suspended in water. After Büchner filtration VI was obtained as a white powder $(30.16 \mathrm{~g}, 0.12 \mathrm{~mol})$ that slowly becomes yellowish over days.

${ }^{1} \mathrm{H}$ NMR $\left(300 \mathrm{MHz}, \mathrm{CDCl}_{3}\right):\{10.0-8.00\}(\mathrm{br}, 1 \mathrm{H}) 7.68\left(\mathrm{~d},{ }^{3} \mathrm{~J}=7.57 \mathrm{~Hz}, 2 \mathrm{H}\right) 7.62\left(\mathrm{t},{ }^{3} \mathrm{~J}=7.57\right.$ $\mathrm{Hz}, 2 \mathrm{H}) 7.35(\mathrm{~s}, 1 \mathrm{H}) 7.08\left(\mathrm{~d},{ }^{3} \mathrm{~J}=7.57 \mathrm{~Hz}, 2 \mathrm{H}\right) 2.60(\mathrm{~s}, 6 \mathrm{H})$

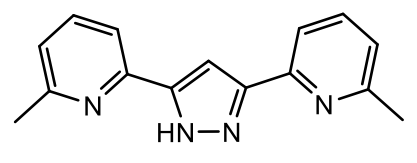

VI

$250,30 \mathrm{~g} \cdot \mathrm{mol}^{-1}$
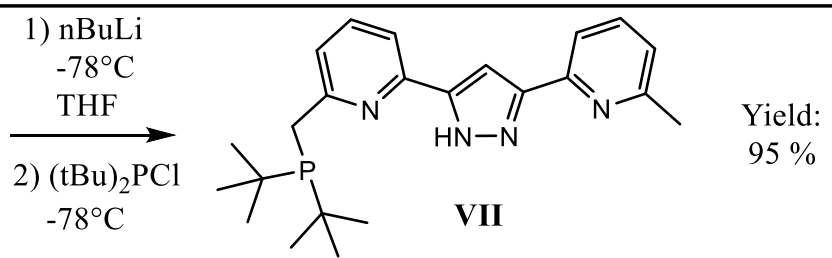

The synthesis was performed according to literature procedure: ${ }^{14}$ the reaction was carried out under argon atmosphere. Compound VI $(2 \mathrm{~g}, 8.0 \mathrm{mmol})$ was dissolved in $20 \mathrm{~mL}$ dry THF. Then nBuLi $2.5 \mathrm{M}$ in hexanes $(8 \mathrm{~mL}, 2.5 \mathrm{eq})$ was dropwise added with a plastic syringe to the lightyellow solution at $-78^{\circ} \mathrm{C}$. After the addition of 1.0 equivalent of $\mathrm{nBuLi}$, the solution turned dark deep red. The other 1.5 equivalent of nBuLi were added faster. When the addition was finished, the solution was allowed to warm up to room temperature whereby a red and white precipitate formed. After 2 hours stirring, the mixture was cooled down again to $-78^{\circ} \mathrm{C}$ and $(\mathrm{tBu})_{2} \mathrm{PCl}(1464$ $\mathrm{mg}, 1 \mathrm{eq}$ ) was added in one portion with a syringe. The precipitate previously formed dissolved, giving a dark red solution. After the solution was allowed to return to ambient temperature, another light red precipitate formed in the following 20 minutes. It was stirred for two hours and then, the reaction was quenched with degassed water $(20 \mathrm{~mL})$ whereby the dark deep red coulour turned yellowish-orange. The mixture was transferred in a dropping funnel that was conditioned with argon in order to separate the two layers. The $20 \mathrm{~mL}$ organic phase was recovered in a 250 mL Schlenk and the solvent was removed under vacuum where the VII was obtained as an oxygen sensitive fluffy light-yellow powder $(4.089 \mathrm{~g}, 7.59 \mathrm{mmol})$.

${ }^{1} \mathrm{H}$ NMR (300 MHz, CO(CD $\left.)_{2}\right): 12.73(\mathrm{br}, 1 \mathrm{H})\left\{7.83\right.$ - 7.68\} (br, 4H) 7.43 (s, 1H) $7.22\left(\mathrm{~d},{ }^{3} \mathrm{~J}=\right.$ $7.38 \mathrm{~Hz}, 1 \mathrm{H}) 7.01\left(\mathrm{~d},{ }^{3} \mathrm{~J}=7.17 \mathrm{~Hz}, 1 \mathrm{H}\right) 3.14\left(\mathrm{~d},{ }^{3} \mathrm{~J}=2.90 \mathrm{~Hz}, 2 \mathrm{H}\right) 2.54(\mathrm{~s}, 3 \mathrm{H}) 1.18\left(\mathrm{~d},{ }^{3} \mathrm{~J}_{\mathrm{H}-\mathrm{P}}=\right.$ $10.74 \mathrm{~Hz}, 18 \mathrm{H})$

${ }^{31}$ P NMR (121 MHz, CO(CD $\left.)_{2}\right): 36.21(\mathrm{br})$ 


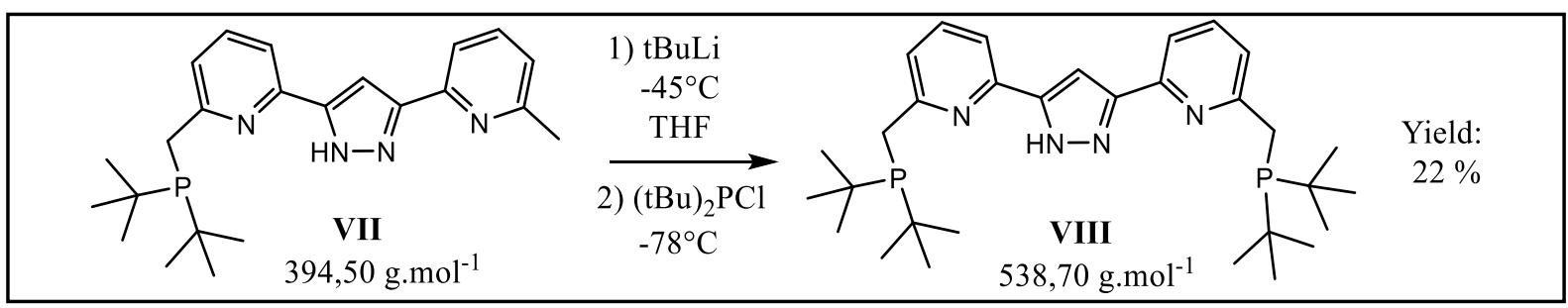

The synthesis was performed according to literature procedure: ${ }^{114}$ The reaction was carried out under argon atmosphere. Compound VII (4089 mg, $7.59 \mathrm{mmol}$ ) was dissolved in $20 \mathrm{~mL}$ dry THF. tBuLi $1.9 \mathrm{M}$ in pentane $(10 \mathrm{~mL}, 2.5 \mathrm{eq})$ were slowly added with a $24 \mathrm{~mL}$ plastic syringe at $-45^{\circ} \mathrm{C}$. After 1 eq tBuLi was added, the light-yellow solution turned dark deep red. After the rest of $\mathrm{tBuLi}$ was added, it was stirred at $-45^{\circ} \mathrm{C}$ for 6 hours and 12 additional hours at ambient temperature, whereby a red precipitate slowly formed. $(\mathrm{tBu})_{2} \mathrm{PCl}(1.390 \mathrm{~g}, 1 \mathrm{eq})$ was added at $-78^{\circ} \mathrm{C}$ in one portion with a plastic syringe and the red precipitate dissolved. The dark deep red solution was allowed to warm up to room temperature and was stirred for 12 hours. After that it was quenched with degassed water, whereby the dark deep red colour turned to yellowish-orange. The mixture was transferred in a dropping funnel that was used to separate the two layers. The organic phase was recovered in a Schlenk and the volatile were removed under vacuum. An orange-red solid was obtained and was dried under vacuum for 6 hours. The orange solid was transferred in the glovebox. The solid was washed with two portions of $20 \mathrm{~mL}$ of $\operatorname{dry~}^{2} \mathrm{t}_{2} \mathrm{O}$ and VIII was finally filtrated and isolated as a pure white powder (900 mg, $1.66 \mathrm{mmol}$ ).

${ }^{1} \mathrm{H}$ NMR (300 MHz, CDCl $): 11.07(\mathrm{br}, 1 \mathrm{H}) 7.81\left(\mathrm{~d},{ }^{3} \mathrm{~J}=7.81 \mathrm{~Hz}, 1 \mathrm{H}\right) 7.64\left(\mathrm{t},{ }^{3} \mathrm{~J}=7.81 \mathrm{~Hz}, 2 \mathrm{H}\right)$ $7.46\left(\mathrm{~d},{ }^{3} \mathrm{~J}=7.81 \mathrm{~Hz}, 1 \mathrm{H}\right) 7.39\left(\mathrm{~d},{ }^{3} \mathrm{~J}=7.81 \mathrm{~Hz}, 1 \mathrm{H}\right) 7.33\left(\mathrm{~d},{ }^{3} \mathrm{~J}=7.81 \mathrm{~Hz}, 1 \mathrm{H}\right) 7.31(\mathrm{~s}, 1 \mathrm{H}) 3.15$ $\left(\mathrm{d},{ }^{2} \mathrm{~J}_{\mathrm{H}-\mathrm{P}}=3.28 \mathrm{~Hz}, 2 \mathrm{H}\right) 3.08\left(\mathrm{~d},{ }^{2} \mathrm{~J}_{\mathrm{H}-\mathrm{P}}=3.28 \mathrm{~Hz}, 2 \mathrm{H}\right) 1.19\left(\mathrm{~d},{ }^{3} \mathrm{~J}_{\mathrm{H}-\mathrm{P}}=11.02 \mathrm{~Hz}, 36 \mathrm{H}\right)$.

${ }^{31} \mathrm{P}$ NMR (121 MHz, $\left.\mathrm{CDCl}_{3}\right): 37.77$ (s) 36.60 (s).

ATR-IR (solid) v $\left(\mathrm{cm}^{-1}\right): 3440(\mathrm{~N}-\mathrm{H}), 1567(\mathrm{C}=\mathrm{N}), 1551(\mathrm{C}=\mathrm{N})$.

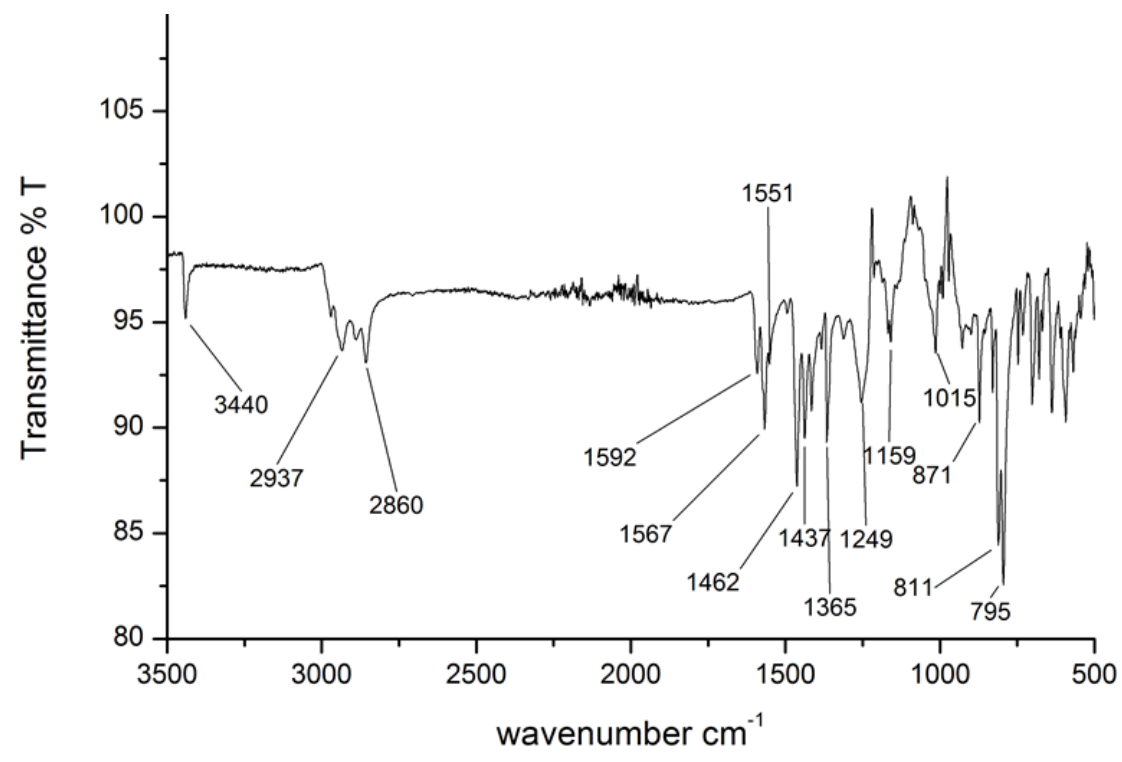

ES1. ATR-IR (solid) of the ligand VIII. 


\section{Complex synthesis}

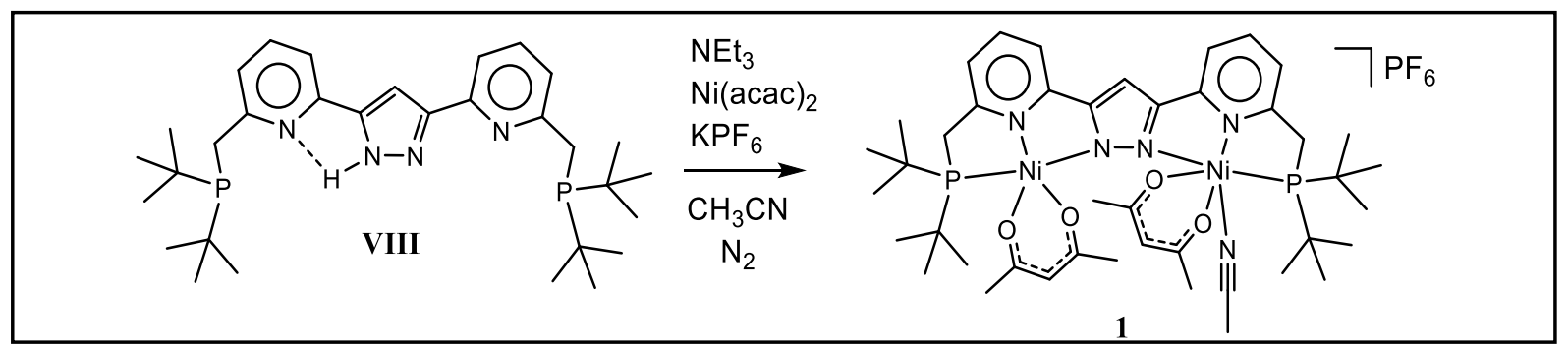

$\left[\mathrm{LH}_{2} \mathrm{Ni}_{2}(\mathrm{acac})_{2}\left(\mathrm{CH}_{3} \mathrm{CN}\right)\right] \mathrm{PF}_{6}(\mathbf{1})$ : The ligand VIII (100 mg, $\left.0.186 \mathrm{mmol}, 1.0 \mathrm{eq}\right)$ was suspended in $1 \mathrm{~mL}$ dry acetonitrile, and the subsequent addition of a solution of $\mathrm{Ni}(\mathrm{acac})_{2}(100,1 \mathrm{mg}, 0.389$ mmol, $2.1 \mathrm{eq})$ in acetonitrile lead to complete dissolution of the ligand powder with a colour change to green. After addition of triethylamine ( $3 \mathrm{eq}$ ), the colour of the solution became deeper green. $\mathrm{KPF}_{6}(102.5 \mathrm{mg}, 0.557 \mathrm{mmol}, 3 \mathrm{eq})$ was added and the solution was stirred for several hours. It was filtered and set to diffusion with diethyl ether whereby the complex $\mathbf{1}$ crystallized after several weeks as deep green oxygen sensitive crystals suitable for X-ray diffraction analysis. The yield was not determined.

Elemental formula: $\mathrm{C}_{41} \mathrm{H}_{61} \mathrm{~F}_{6} \mathrm{~N}_{4} \mathrm{Ni}_{2} \mathrm{O}_{4} \mathrm{P}_{3}$

Molecular weight: $998.26 \mathrm{~g} \cdot \mathrm{mol}^{-1}$

${ }^{1} \mathrm{H}$ NMR (400 MHz, CD $\mathrm{Cl}_{2}, 298$ K): 57.29 (br, 2H, CH-Py) 47.58 (br, 2H, CH-Py) 46.33 (br, 1H, CH-Pz) 38.40 (br, 4H, CH$\left.{ }_{2}\right) 13.13$ (br s, 36H, CH${ }_{3}{ }^{\circ} \mathrm{Bu}$ ) 11.87(br, 2H, CH-Py) 2.15 (br, 12H, $\left.\mathrm{CH}_{3}\right)-28.99$ (br, 2H, CH).

${ }^{31}$ P NMR (162 MHz, $\left.\mathrm{CD}_{2} \mathrm{Cl}_{2}, 233 \mathrm{~K}\right): 93.12,-144\left(\mathrm{PF}_{6}\right)$.

${ }^{13} \mathrm{C}$ NMR (125 MHz, CD $\mathrm{Cl}_{2}, 298$ K): 629.94 (br) 608.98 (br) 596.42 (br) 191.47 (br) 154.69 (br) 150.49 (br) 149.57 (br) 148.27 (br) 130.64 (br) 129.26 (br) 126.91 (br) 65.66 (br) 15.62 (br) 14.63 (br) 3.09 (br) 3.02 (br) -173.86 (br).

MS-ESI (+), MeCN) $m / z: 851.3:\left[\mathrm{LH}_{2} \mathrm{Ni}_{2}(\mathrm{acac})_{2}\right]^{+}$

IR $(\mathrm{ATR}) v\left(\mathrm{~cm}^{-1}\right): 1600(\mathrm{C}=\mathrm{O}), 1590,1571(\mathrm{C}=\mathrm{N})(\mathrm{cf}$ Figure 3.10).

UV/Vis VT: $\lambda_{\max }(\mathrm{nm}) 313,262$.

Elemental Analysis (\%): Found: C, 35.30; H, 4.79; N, 4.04. 


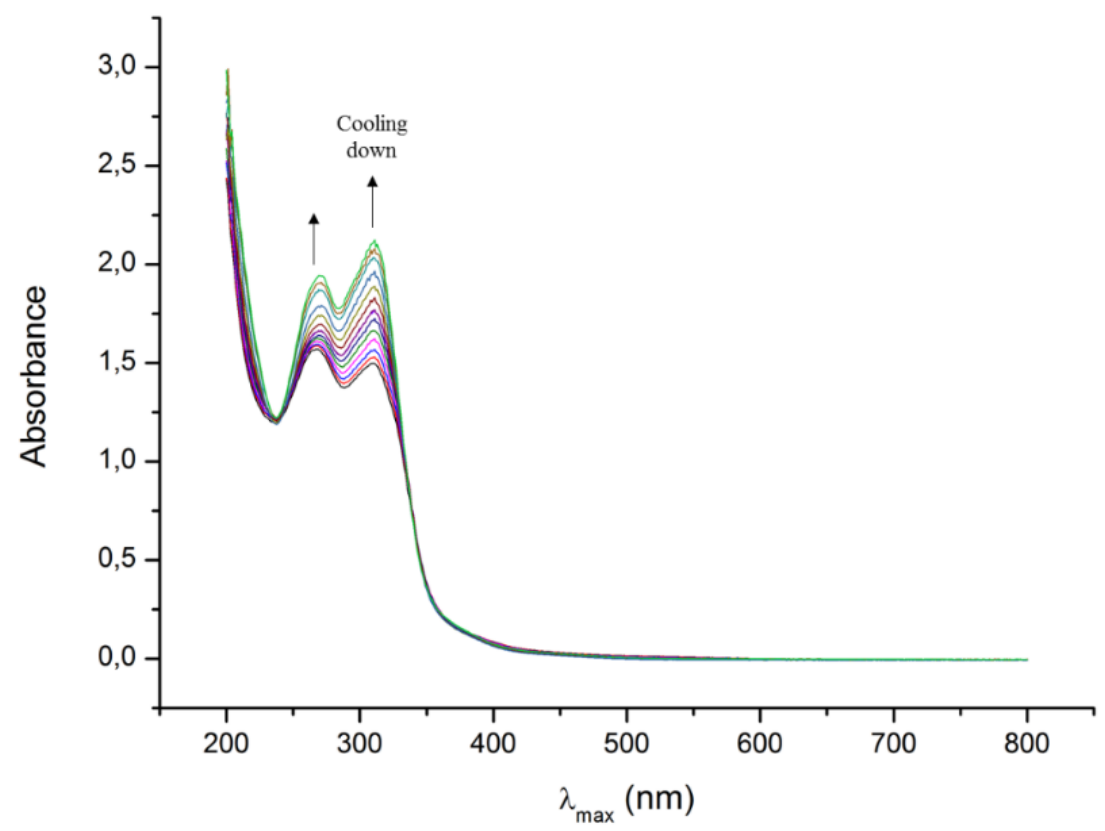

ES2. VT UV-Vis of complex 1 in $\mathrm{CH}_{3} \mathrm{CN}$ from 238 to $338 \mathrm{~K}$.

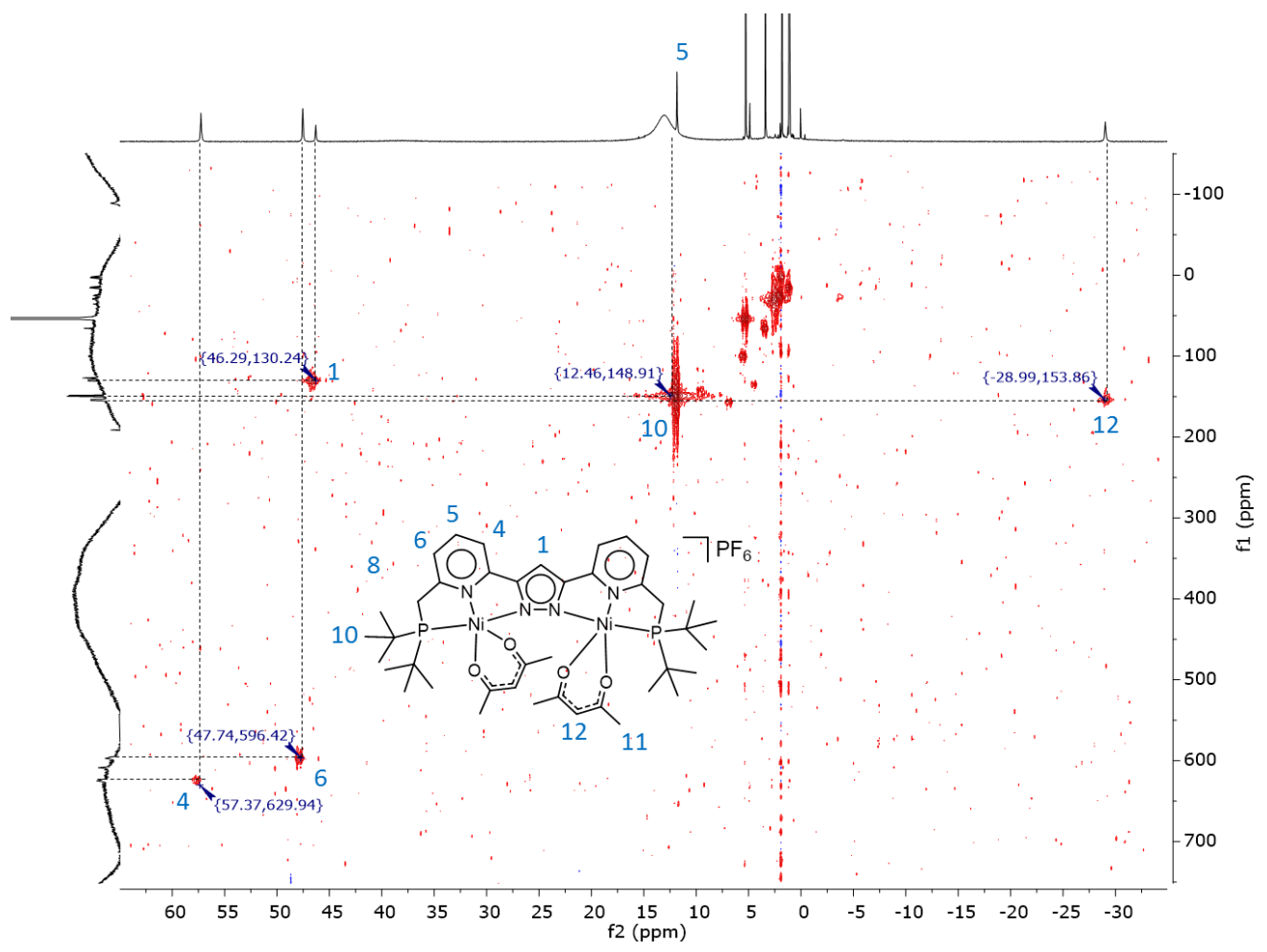

ES3. ${ }^{1} \mathrm{H} /{ }^{13} \mathrm{C}$ HSQC of complex $\mathbf{1}$ at $348 \mathrm{~K}$ in $\mathrm{CD}_{3} \mathrm{CN}, 500 \mathrm{MHz}$. 


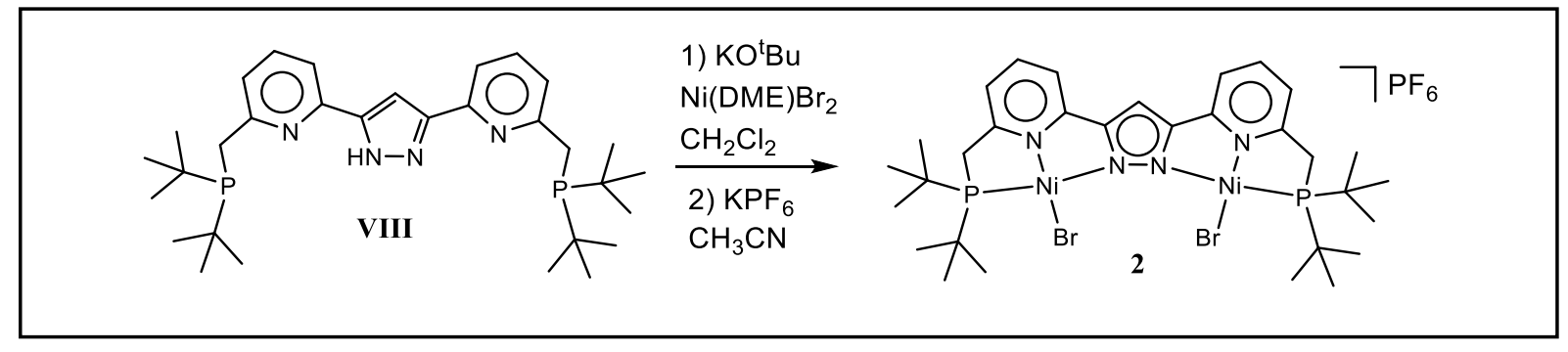

[ $\left.\mathrm{LH}_{2} \mathrm{Ni}_{2} \mathrm{Br}_{2}\right] \mathrm{PF}_{6}$ (2): The ligand VIII (400 mg, $0.74 \mathrm{mmol}, 1.0$ equiv) was dissolved in $10 \mathrm{~mL}$ dry dichloromethane. The suspension was stirred until the white powder was completely dissolved. To the resulting light yellow solution was added $\mathrm{Ni}(\mathrm{DME}) \mathrm{Br}_{2}$ (688 mg, $2.2 \mathrm{mmol}, 3.0$ equiv) in one portion. A color change via light red to deep red in the following minutes was observed. $\mathrm{KO} \mathrm{B}^{\mathrm{Bu}}(83.4 \mathrm{mg}, 0.71 \mathrm{mmol}, 0.95$ equiv) was then added and the solution was stirred for 12 hours. The volatiles were removed and the residue was redissolved in dry acetonitrile to which $\mathrm{KPF}_{6}$ (684.3 mg, $3.7 \mathrm{mmol}, 5$ equiv) was added and stirred for further 12 hours. The precipitate was filtered through a glas fiber filter and removed while the deep red solution was set to vapor diffusion with dry pentane. Black red crystals were obtained after three days in $84 \%$ yield.

Elemental formula: $\mathrm{C}_{31} \mathrm{H}_{47} \mathrm{Br}_{2} \mathrm{~F}_{6} \mathrm{~N}_{4} \mathrm{Ni}_{2} \mathrm{P}_{3}$

Molecular weight: 959.85 g.mol ${ }^{-1}$

${ }^{1} \mathrm{H}$ NMR (400 MHz, $\mathrm{CD}_{2} \mathrm{Cl}_{2}$ ), 8.18 (br d, 2 H, CH-Py) 7.97 (t, 2 H, CH-Py) 7.86 (br d, 2 H, CHPy) 7.75 (s, $1 \mathrm{H}, \mathrm{CH}-\mathrm{Pz}) 3.54$ (br, $\left.4 \mathrm{H}, \mathrm{CH}_{2}\right) 1.74$ (br s, $36 \mathrm{H}, \mathrm{CH}_{3}{ }^{\mathrm{t}} \mathrm{Bu}$ ).

${ }^{13} \mathrm{C}$ NMR (126 MHz, CD $\left.\mathrm{Cl}_{2}, 268 \mathrm{~K}\right), 160.43$ (br) 155.87 (br) 149.71 (br) 141.48 (br) 123.77 (br) 120.78 (br) 100.91 (br) 38.89 (br) 33.53 (br) 29.89 (br).

${ }^{31}$ P NMR (162 MHz, $\left.\mathrm{CD}_{2} \mathrm{Cl}_{2}, 218 \mathrm{~K}\right) 76.90$ (br s), -144 (hept).

MS-ESI (+), MeCN) $m / z: 815:\left[\mathrm{LH}_{2} \mathrm{Ni}_{2} \mathrm{Br}_{2}\right]^{+}$

IR (ATR) v/ $\mathrm{cm}^{-1}: 1610,1560$ (cf Figure 3.10).

UV/Vis: $\lambda_{\max }(\mathrm{nm}) 282,329,495$.

Elemental Analysis (\%) : Calculated for $\mathrm{C}_{31} \mathrm{H}_{49} \mathrm{Br}_{2} \mathrm{~F}_{6} \mathrm{~N}_{4} \mathrm{Ni}_{2} \mathrm{OP}_{3}$ (including 1. $\mathrm{H}_{2} \mathrm{O}$ ): $\mathrm{C}, 37.90 ; \mathrm{H}$, 5.29; N, 5.76 Found: C, 38.08; H, 5.05; N, 5.77. 


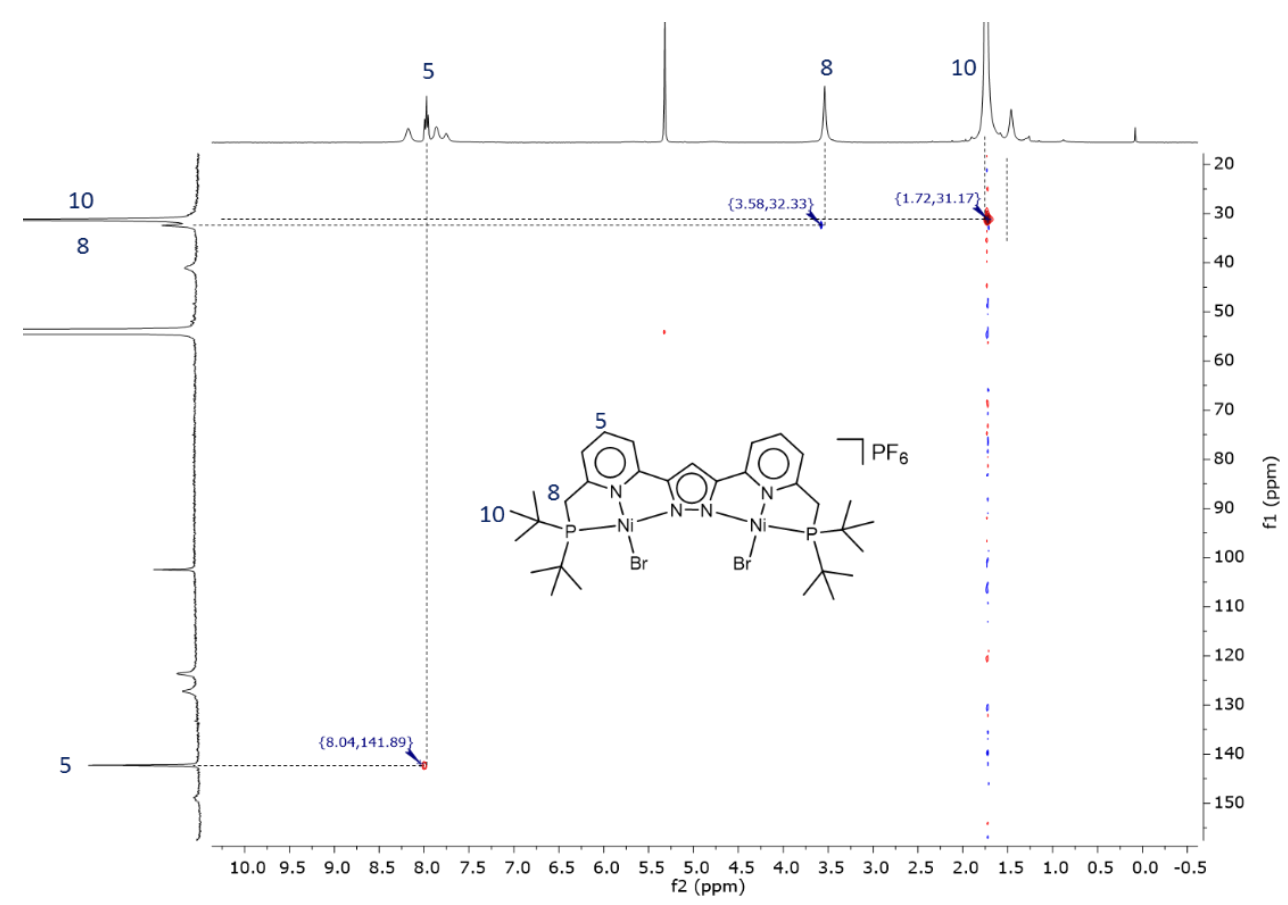

ES4. ${ }^{1} \mathrm{H} /{ }^{13} \mathrm{C}$ HSQC of complex 2 at $248 \mathrm{~K}$ in $\mathrm{CD}_{2} \mathrm{Cl}_{2}, 500 \mathrm{MHz}$.

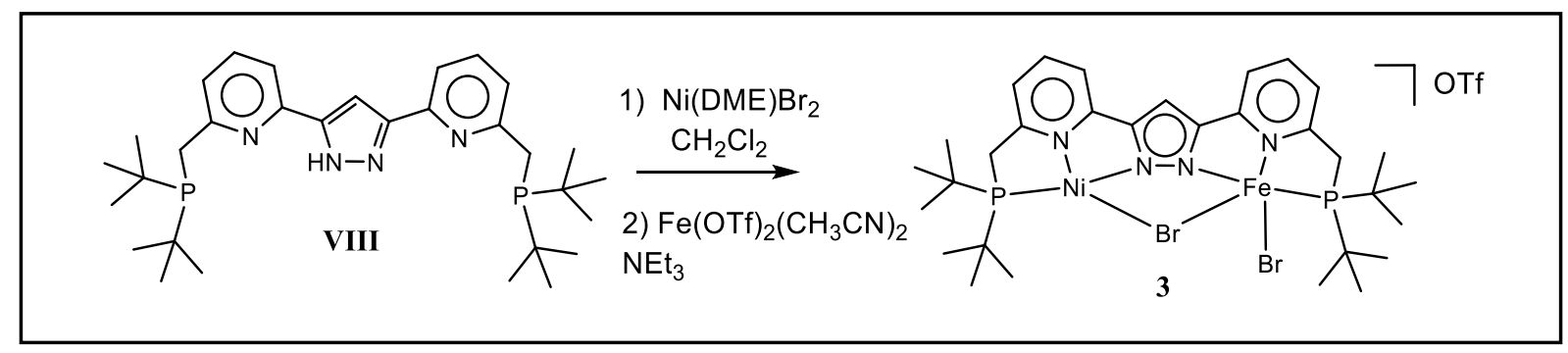

[ $\left.\mathrm{LH}_{2} \mathrm{Ni}(\boldsymbol{\mu}-\mathrm{Br}) \mathrm{FeBr}\right] \mathrm{OTf}(\mathbf{3})$ : The ligand VIII $(100 \mathrm{mg}, 0.186 \mathrm{mmol}, 1.0$ equiv) was dissolved in 1 $\mathrm{mL}$ dry methylene chloride and one equivalent of $\mathrm{Ni}(\mathrm{DME}) \mathrm{Br}_{2}(57.3 \mathrm{mg}, 0.186 \mathrm{mmol}, 1.0$ equi) was slowly added. The suspension turned red in the following minutes. After one hour stirring, one equivalent of $\mathrm{Fe}(\mathrm{OTf})_{2}\left(\mathrm{CH}_{3} \mathrm{CN}\right)_{2}\left(81.1 \mathrm{mg}, 0.186 \mathrm{mmol}, 1.0\right.$ equiv) dissolved in $\mathrm{CH}_{3} \mathrm{CN}$ was added, the solution turned brown and triethylamine was added. A yellow precipitate of $\left(\mathrm{Et}_{3} \mathrm{NH}\right)(\mathrm{OTf})$ formed. It was filtrated, and the remaining solution was set to vapor diffusion with diethyl ether. Complex $\mathbf{4}$ was obtained as black single crystals suitable for X-ray diffraction. The yield was not determined.

Elemental formula: $\mathrm{C}_{32} \mathrm{H}_{47} \mathrm{Br}_{2} \mathrm{~F}_{3} \mathrm{FeN}_{4} \mathrm{NiO}_{3} \mathrm{P}_{2} \mathrm{~S}$

Molecular weight: $961.10 \mathrm{~g} \cdot \mathrm{mol}^{-1}$

${ }^{1} \mathrm{H}$ NMR (400 MHz, $\mathrm{CD}_{2} \mathrm{Cl}_{2}$ ): 66.76 (br) 62.74 (br) 36.55 (br) 13.19 (br) 10.39 (br) 3.39 (br) 2.58 (br) -2.57 (br) -4.40 (br) -8.55 (br).

MS-ESI (+), MeCN) $m / z: 809.03:\left[\mathrm{LH}_{2} \mathrm{Ni}(\mu-\mathrm{Br}) \mathrm{FeBr}\right]^{+}$

UV/Vis VT: $\lambda_{\max }(\mathrm{nm}) 318,265$ 


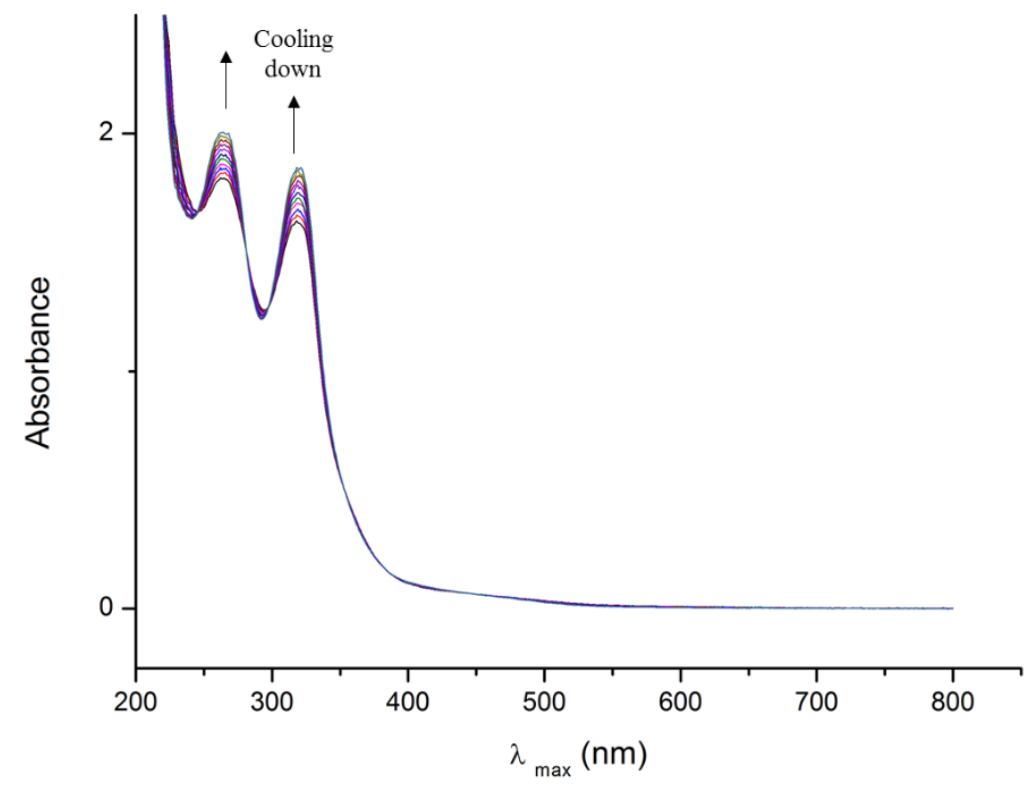

ES5. VT UV-Vis of complex 3 in $\mathrm{CH}_{3} \mathrm{CN}$ from 238 to $338 \mathrm{~K}$.

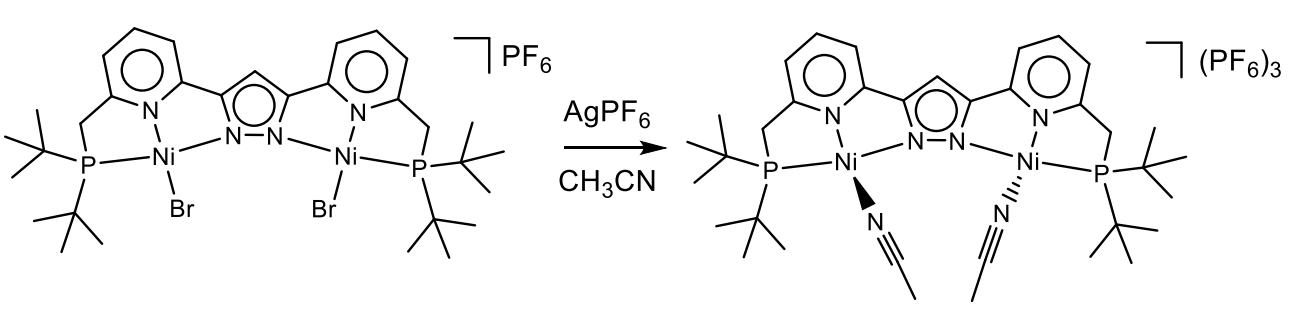

$\left[\mathrm{LH}_{2} \mathrm{Ni}_{2}\left(\mathrm{CH}_{3} \mathrm{CN}\right)_{2}\right]\left(\mathrm{PF}_{6}\right)_{3}(\mathbf{4}):\left[\mathrm{LNi}_{2} \mathrm{Br}_{2}\right] \mathrm{PF}_{6}(100 \mathrm{mg}, 0.104 \mathrm{mmol}, 1$ equiv) was dissolved in $\mathrm{CH}_{3} \mathrm{CN}$. After addition of $\mathrm{AgPF}_{6}(55 \mathrm{mg}, 0.218 \mathrm{mmol}, 2.1$ equiv), the red solution immediately turned into light orange and a precipitate of $\mathrm{AgBr}$ formed. The mixture was stirred for $30 \mathrm{~min}$ and was filtrated. The filtrate was set to vapor diffusion with $\mathrm{Et}_{2} \mathrm{O}$, whereby suitable crystals for X-ray diffraction were obtained. The yield was not determined.

Elemental formula: $\mathrm{C}_{35} \mathrm{H}_{53} \mathrm{~F}_{18} \mathrm{~N}_{6} \mathrm{Ni}_{2} \mathrm{P}_{5}$

Molecular weight: 1172.08 g.mol ${ }^{-1}$

${ }^{1} \mathrm{H}$ NMR (500 MHz, CD 3 CN, 348 K): 9.62 (br, 2H) 9.41 (br, 3H) 8.47 (br, 2H) 5.20 (br, 4H, $\left.\mathrm{CH}_{2}\right) 1.98$ (br, 36H, tBu).

${ }^{13} \mathrm{C}$ NMR (126 MHz, CD ${ }_{3} \mathrm{CN}, 348$ K): 171.71 (br) 157.00 (br) 142.86 (br) 141.52 (br) 138.30 (br) 132.56 (br) 108.76 (br) 52.89 (br) 33.79 (br) 22.31 (br).

${ }^{31} \mathrm{P}$ NMR (205 MHz, CD 3 CN, 348/248 K): -144 $\left(\mathrm{PF}_{6}\right)$

$\mathrm{UV} / \mathrm{Vis} \mathrm{VT}: \boldsymbol{\lambda}_{\max }(\mathrm{nm}) 447,325,274$. 


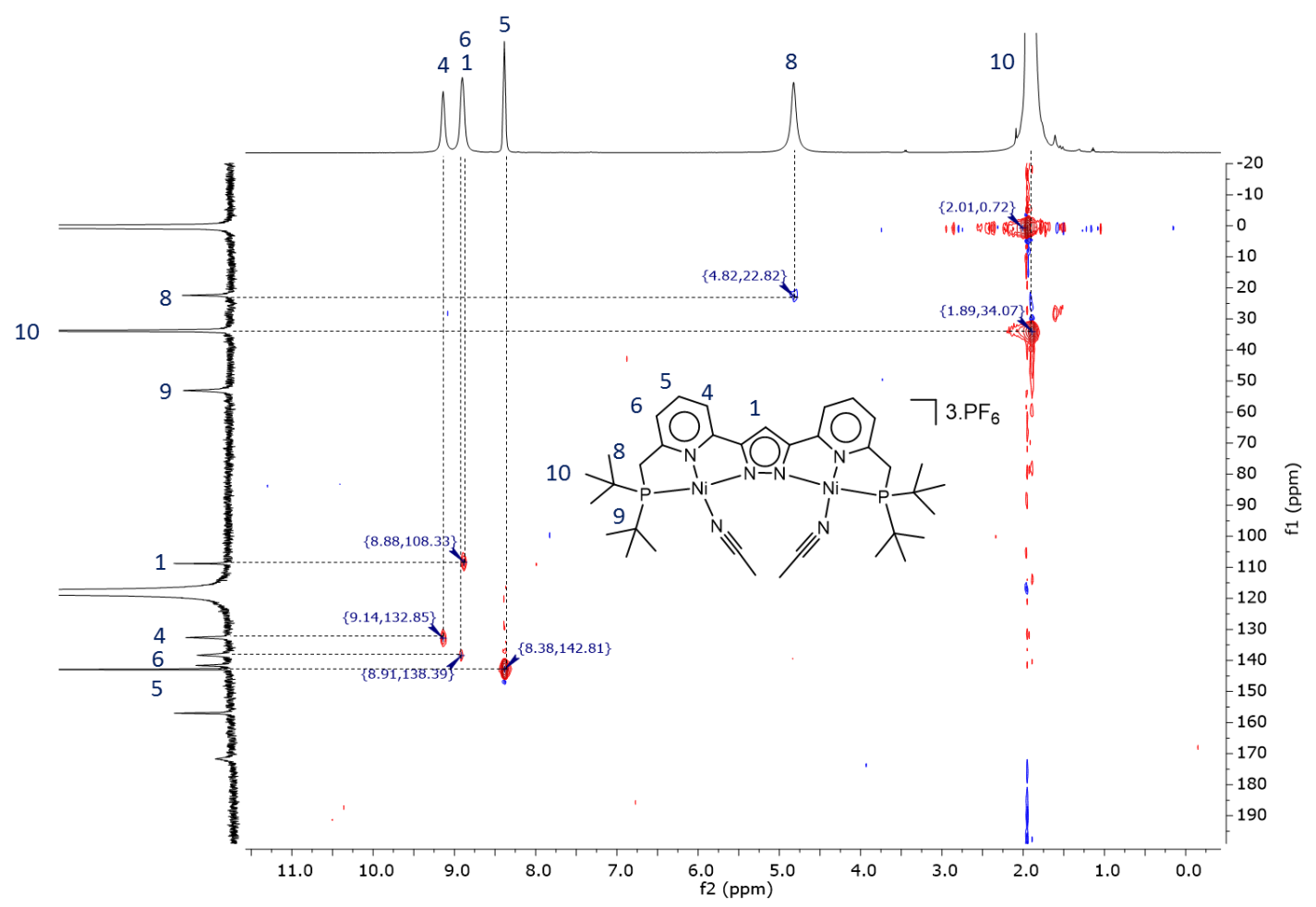

ES6. ${ }^{1} \mathrm{H} /{ }^{13} \mathrm{C}$ HSQC of complex 4 at $348 \mathrm{~K}$ in $\mathrm{CD}_{3} \mathrm{CN}, 500 \mathrm{MHz}$.

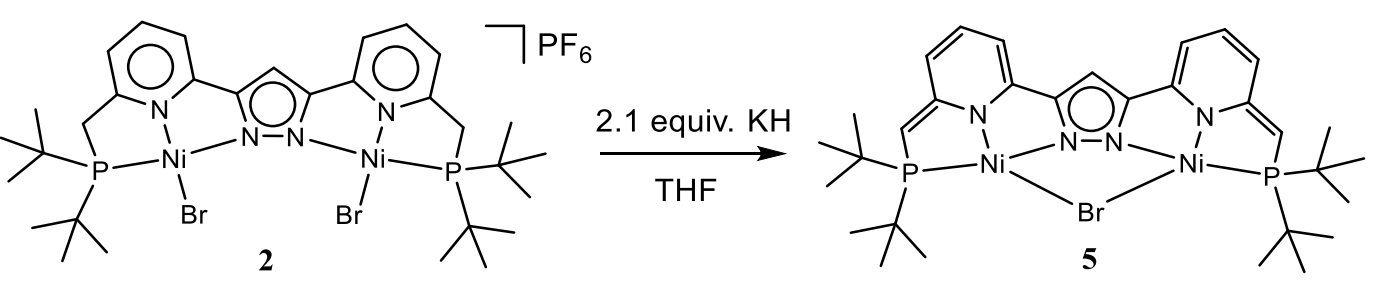

[ $\left.\mathrm{LNi}_{2}(\mathbf{\mu}-\mathrm{Br})\right](\mathbf{5})$ : Complex 2 (100 mg, $0.104 \mathrm{mmol}, 1$ equiv) was suspended in THF. KH (8.7 mg, $0.218 \mathrm{mmol}, 2.1$ equiv) was added and the mixture was vigorously stirred for 12 hours. A slow bubbling and colour change from red to deep violet evidenced the deprotonation. The solution was filtrated, and the volatiles were removed under vacuum affording the doubly dearomatized complex $\mathbf{5}$. The yield was not determined.

${ }^{1} \mathrm{H}$ NMR $\left(500 \mathrm{MHz}\right.$, THF-d $\left.{ }^{8}\right): 6.36\left(\mathrm{ddd},{ }^{3} \mathrm{~J}=8.92 \mathrm{~Hz},{ }^{3} \mathrm{~J}=6.56 \mathrm{~Hz},{ }^{5} \mathrm{~J}_{\mathrm{P}-\mathrm{H}}=2.27 \mathrm{~Hz}, 2 \mathrm{H}\right) 6.06(\mathrm{~s}$, $1 \mathrm{H}) 5.87\left(\mathrm{~d},{ }^{3} \mathrm{~J}=8.92,2 \mathrm{H}\right) 5.48\left(\mathrm{~d},{ }^{3} \mathrm{~J}=6.56,2 \mathrm{H}\right) 2.83\left(\mathrm{~d},{ }^{3} \mathrm{~J}=3.04 \mathrm{~Hz}, 2 \mathrm{H}\right) 1.46\left(\mathrm{~d},{ }^{3} \mathrm{~J}=13.78\right.$ $\mathrm{Hz}, 36 \mathrm{H})$.

${ }^{13} \mathrm{C}$ NMR (126 MHz, THF-d $\left.{ }^{8}\right): 171.54\left(\mathrm{~d},{ }^{2} \mathrm{~J}=16.95 \mathrm{~Hz}\right) 152.43\left(\mathrm{~d},{ }^{5} \mathrm{~J}=2.67 \mathrm{~Hz}\right) 152.18\left(\mathrm{~d},{ }^{4} \mathrm{~J}=\right.$ $2.67 \mathrm{~Hz}) 133.30$ (s) $115.07\left(\mathrm{~d},{ }^{3} \mathrm{~J}=17.23 \mathrm{~Hz}\right) 99.60$ (s) 95.67 (s) 59.52 (d, $\left.{ }^{1} \mathrm{~J}=61.34 \mathrm{~Hz}\right) 36.46$ $\left(\mathrm{d},{ }^{1} \mathrm{~J}=23.47 \mathrm{~Hz}\right) 29.19\left(\mathrm{~d},{ }^{2} \mathrm{~J}=3.99 \mathrm{~Hz}\right)$

${ }^{31}$ P NMR (202 MHz, THF-d $\left.{ }^{8}\right): 68.06$. 


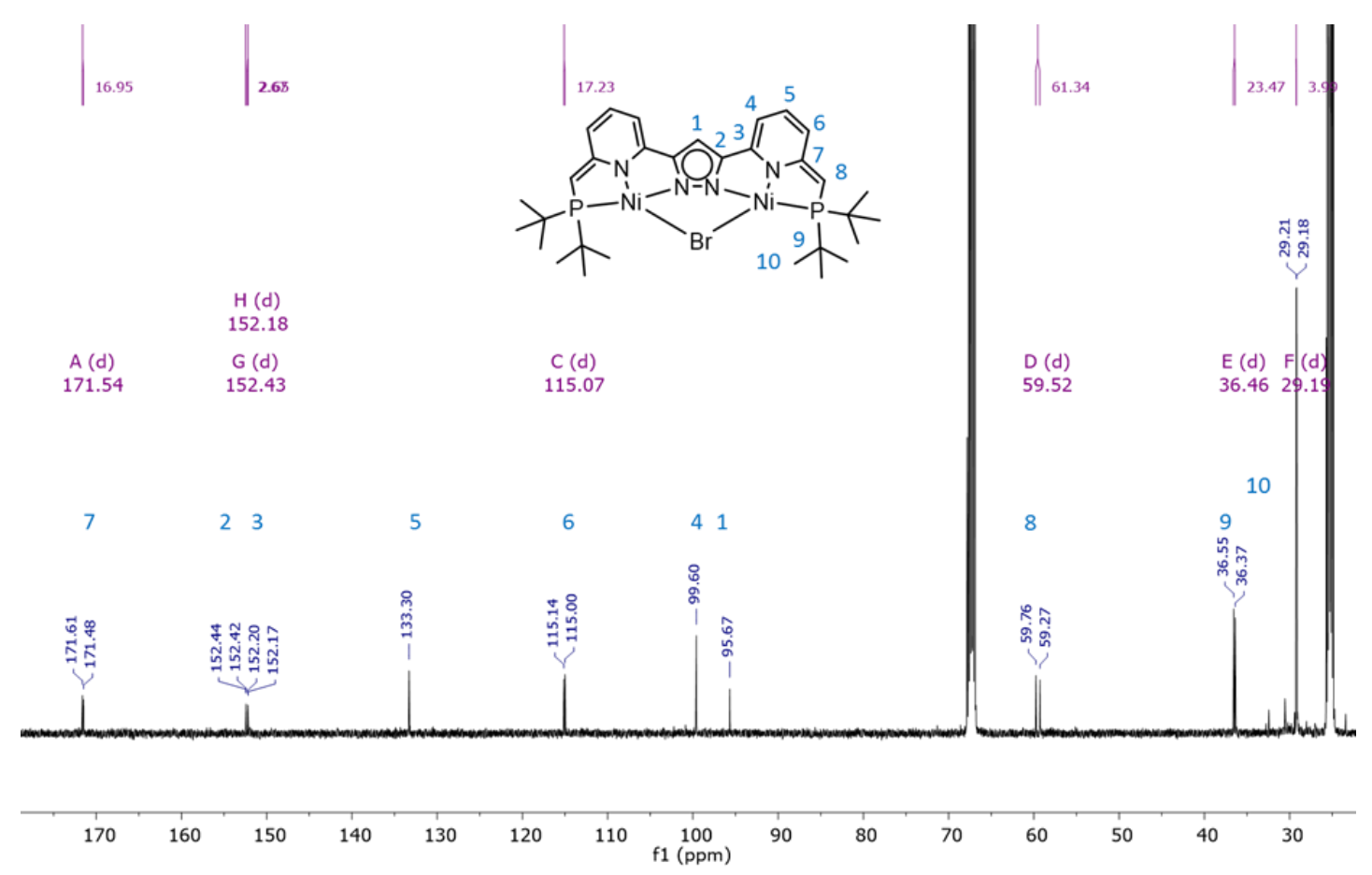

ES7. ${ }^{13} \mathrm{C}$ NMR of complex $\mathbf{5}$ in THF-d ${ }^{8}(126 \mathrm{Mhz})$.

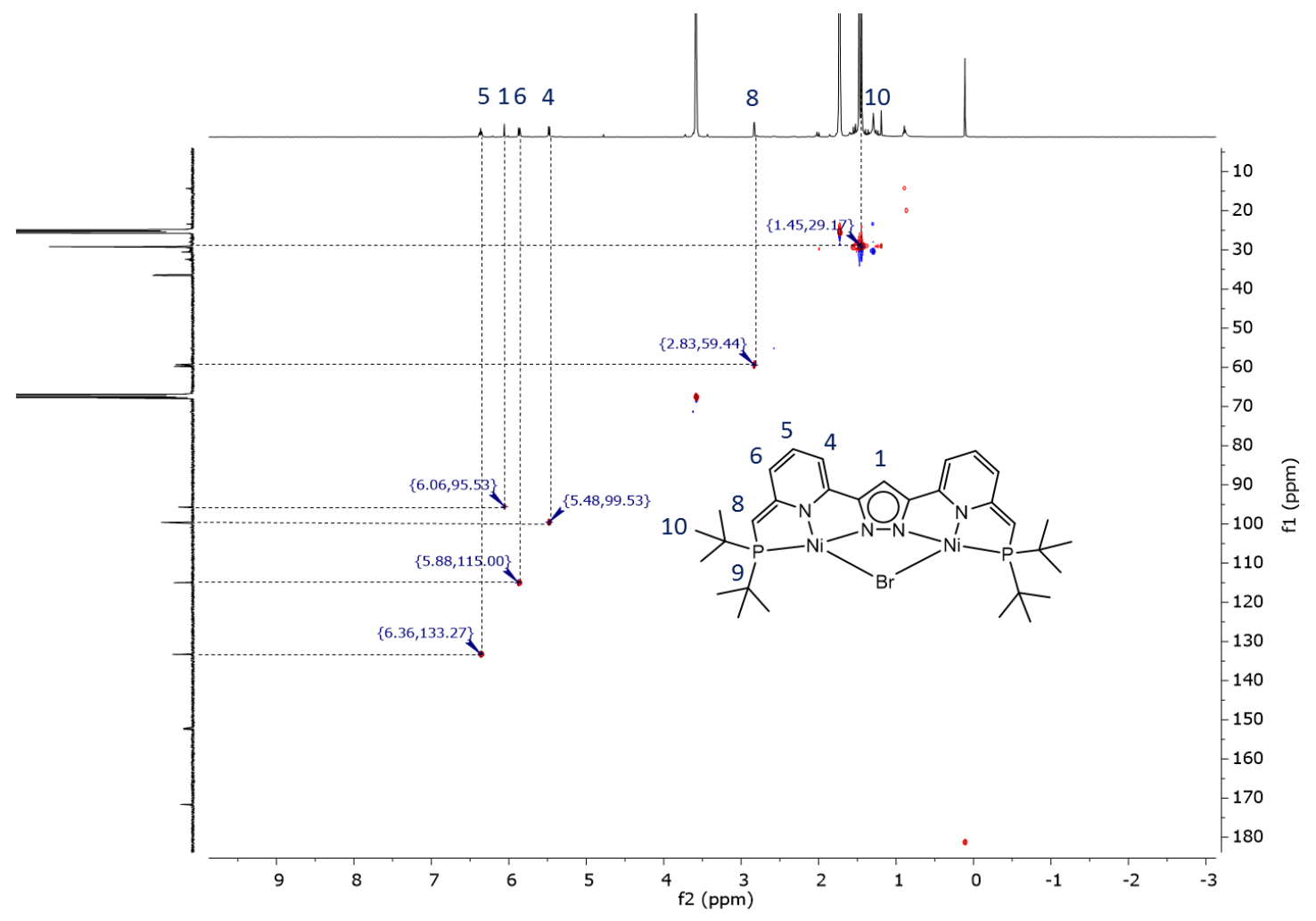

ES8. ${ }^{1} \mathrm{H} /{ }^{13} \mathrm{C}$ HSQC of complex 5 in THF-d ${ }^{8}(500 \mathrm{Mhz})$. 


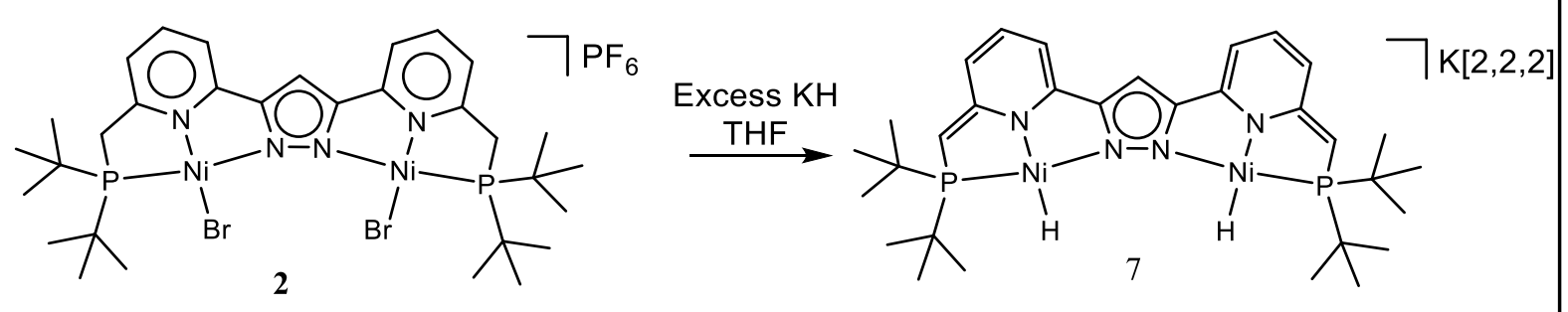

$\mathrm{K}[2,2,2]\left[\mathrm{LNi}_{2}(\mathrm{H})_{2}\right]$ (7): Dark-red crystals of $\left[\mathrm{LNi}_{2} \mathrm{Br}_{2}\right] \mathrm{PF}_{6}(100 \mathrm{mg}, 0.10 \mathrm{mmol}, 1$ equiv) were suspended in $5 \mathrm{~mL}$ of THF. One portion of $\mathrm{KH}$ ( $40 \mathrm{mg}, 1.00 \mathrm{mmol}, 10$ equiv) was added to this suspension resulting in a slight bubbling. After ca 18 hours of stirring, the suspension had turned into a violet solution indicating the formation of the doubly deprotonated complex 2 . Another portion of $\mathrm{KH}$ ( $40 \mathrm{mg}, 1.00 \mathrm{mmol}, 10$ equiv) was added and the solution was further stirred for 48 hours. The precipitate resulting from the mixture was separated via filtration and Cryptant [2,2,2] (38.4 $\mathrm{mg}, 0.10 \mathrm{mmol}, 1$ equiv) was added to the solution. A colour change from violet to Bordeaux was observed. After 6 hours, the solution was set to liquid diffusion with hexane at $-35^{\circ} \mathrm{C}$. Black violet crystals of complex 7 suitable for X-ray diffraction were obtained in the following 12 hours.

Elemental formula: $\mathrm{C}_{49} \mathrm{H}_{83} \mathrm{KN}_{6} \mathrm{Ni}_{2} \mathrm{O}_{6} \mathrm{P}_{2}$

Molecular weight: 1070.67 g.mol ${ }^{-1}$

${ }^{1} \mathrm{H}$ NMR $\left.\left(400 \mathrm{MHz}, \mathrm{THF}^{\mathrm{d}}\right)^{2}\right) 6.28\left(\mathrm{ddd},{ }^{3} \mathrm{~J}=6.80 \mathrm{~Hz},{ }^{3} \mathrm{~J}=8.50 \mathrm{~Hz},{ }^{5} \mathrm{~J}_{\mathrm{P}-\mathrm{H}}=1.80 \mathrm{~Hz}, 2 \mathrm{H}\right), 6.05(\mathrm{~s}$, 1H) $5.73\left(\mathrm{~d},{ }^{3} \mathrm{~J}=8.50 \mathrm{~Hz}, 2 \mathrm{H}\right) 5.35\left(\mathrm{~d},{ }^{3} \mathrm{~J}=6.80 \mathrm{~Hz}, 2 \mathrm{H}\right) 3.57(\mathrm{bs}, 12 \mathrm{H}) 3.50(\mathrm{~m}, 12 \mathrm{H}) 2.77(\mathrm{~d}$, $\left.{ }^{4} \mathrm{~J}_{\mathrm{H} \cdot \mathrm{H}}=2.29 \mathrm{~Hz}, 2 \mathrm{H}\right) 2.49(\mathrm{~m}, 12 \mathrm{H}) 1.34\left(\mathrm{~d},{ }^{3} \mathrm{~J}=12.90 \mathrm{~Hz}, 36 \mathrm{H}\right)-20.19\left(\mathrm{dd},{ }^{2} \mathrm{~J}_{\mathrm{P} \cdot \mathrm{H}}=95.7 \mathrm{~Hz},{ }^{4} \mathrm{~J}_{\mathrm{H} \cdot \mathrm{H}}\right.$ $=2.29 \mathrm{~Hz}, 2 \mathrm{H})$.

${ }^{13} \mathrm{C}$ NMR (126 MHz, THF-d $\left.{ }^{8}\right) 168.34\left(\mathrm{~d},{ }^{2} \mathrm{~J}=18.95 \mathrm{~Hz}\right) 156.84\left(\mathrm{~d},{ }^{4} \mathrm{~J}=3.14 \mathrm{~Hz}\right) 152.46\left(\mathrm{~d},{ }^{3} \mathrm{~J}=\right.$ $3.02 \mathrm{~Hz}) 133.12\left(\mathrm{~d},{ }^{4} \mathrm{~J}=1.93 \mathrm{~Hz}\right) 110.94\left(\mathrm{~d},{ }^{3} \mathrm{~J}=16 \mathrm{~Hz}\right) 95.48$ (s) 94.36 (s) $59.03\left(\mathrm{~d},{ }^{1} \mathrm{~J}=52.20 \mathrm{~Hz}\right)$ $34.70\left(\mathrm{~d},{ }^{1} \mathrm{~J}=25.53 \mathrm{~Hz}\right) 30.24\left(\mathrm{~d},{ }^{2} \mathrm{~J}=4.86 \mathrm{~Hz}\right)$.

${ }^{31}$ P NMR (162 MHz, THF-d $\left.{ }^{8}\right) 84.11(\mathrm{~s})$.

MS-ESI (-), THF) $m / z: 655:\left[\operatorname{LNi}_{2}(\mathrm{H})_{2}\right]^{-}$

IR (ATR) $v / \mathrm{cm}^{-1}: 1847(\mathrm{Ni}-\mathrm{H})$ (cf Figure 3.10).

Elemental Analysis (\%): Calculated for $\mathrm{C}_{53} \mathrm{H}_{90} \mathrm{KN}_{6} \mathrm{Ni}_{2} \mathrm{O}_{7} \mathrm{P}_{2}$ (including 1.THF) C, 55.75; $\mathrm{H}$, 7.95; N, 7.36 Found: C, 55.55; H, 8.00; N, 7.26. 


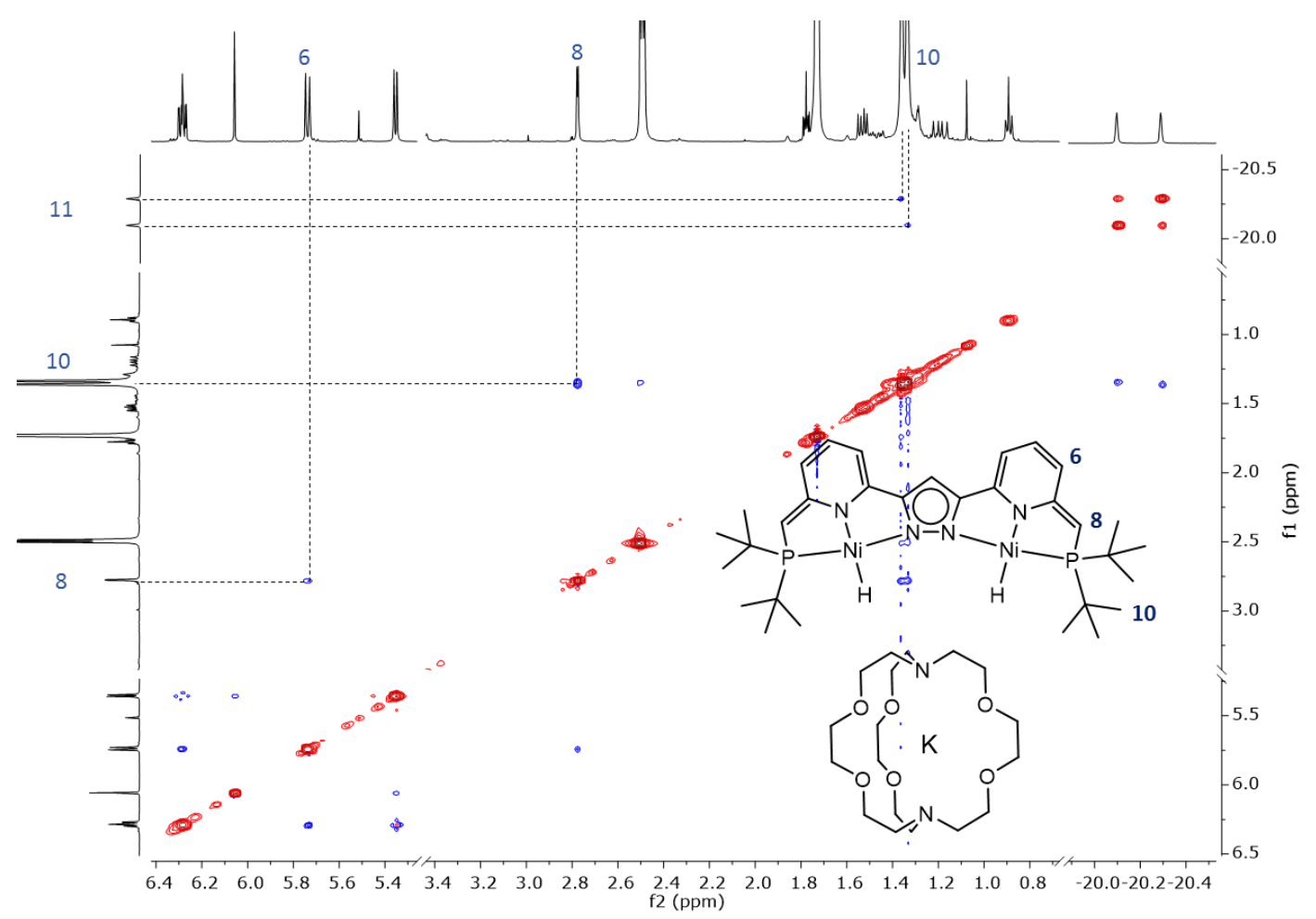

ES9. NOESY of complex 7 in THF-d ${ }^{8}(500 \mathrm{MHz})$.
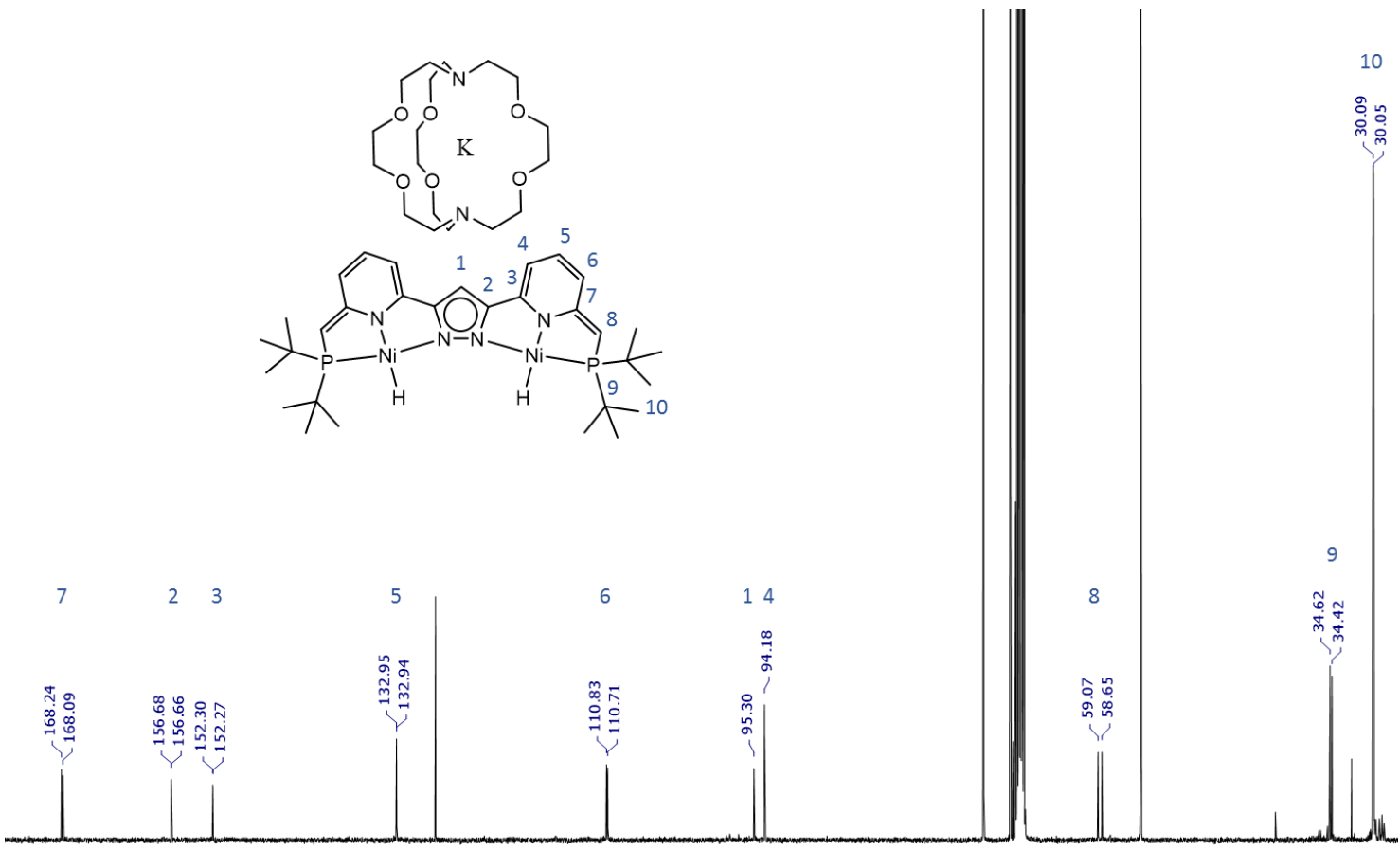

$\begin{array}{lllllllllllllllllllllllllllllllllllllllll}170 & 165 & 160 & 155 & 150 & 145 & 140 & 135 & 130 & 125 & 120 & 115 & 110 & 105 & 100 & 95 & 90 & 85 & 80 & 75 & 70 & 65 & 60 & 55 & 50 & 45 & 40 & 35 & 30\end{array}$

ES10. ${ }^{13} \mathrm{C}$ NMR of complex 7 in $\mathrm{THF}^{8}{ }^{8}(126 \mathrm{MHz})$. 


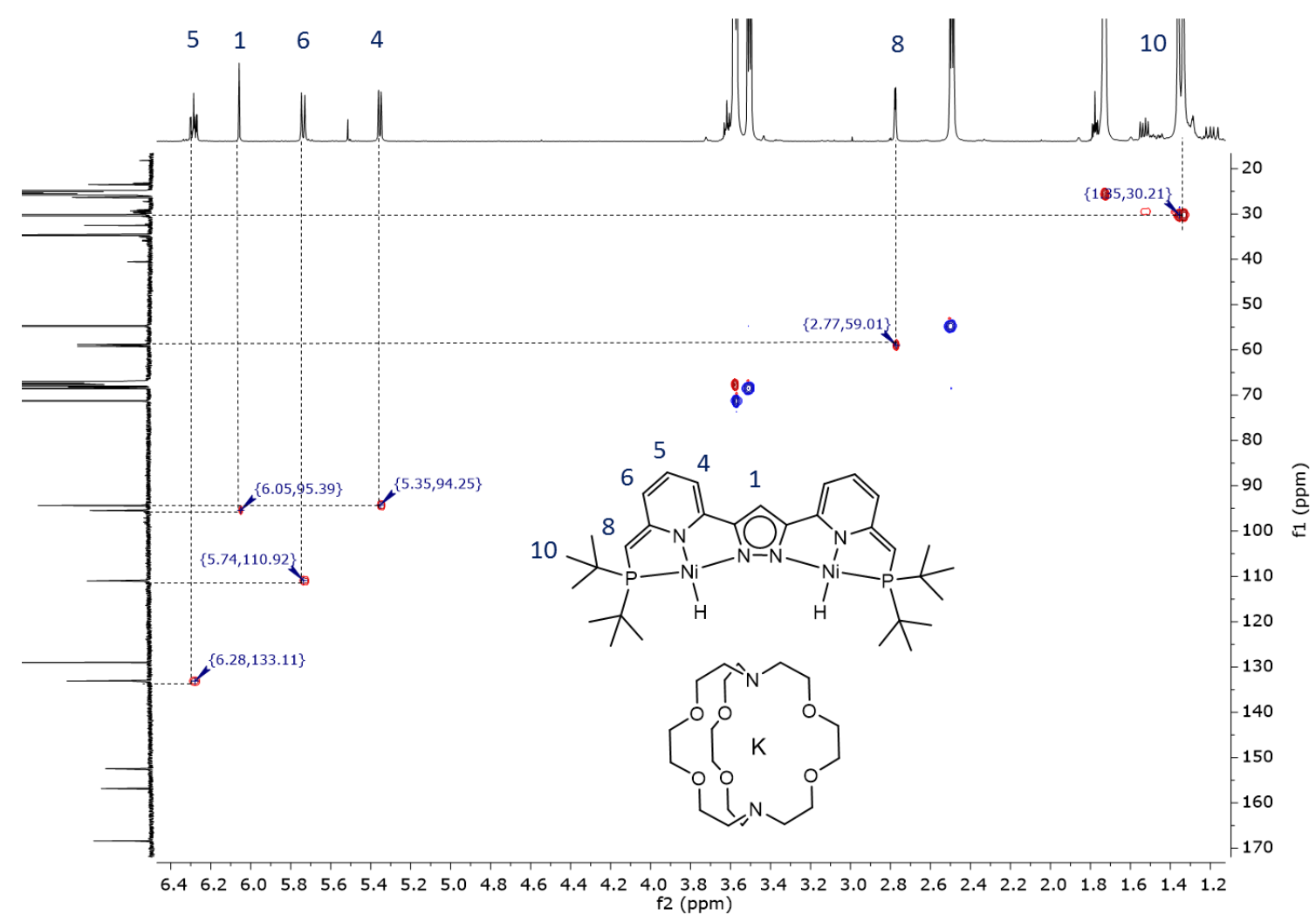

ES11. $\left({ }^{1} \mathrm{H} /{ }^{13} \mathrm{C}\right) \mathrm{HSQC}$ of complex 7 in THF-d $\mathrm{d}^{8}(500 \mathrm{MHz})$

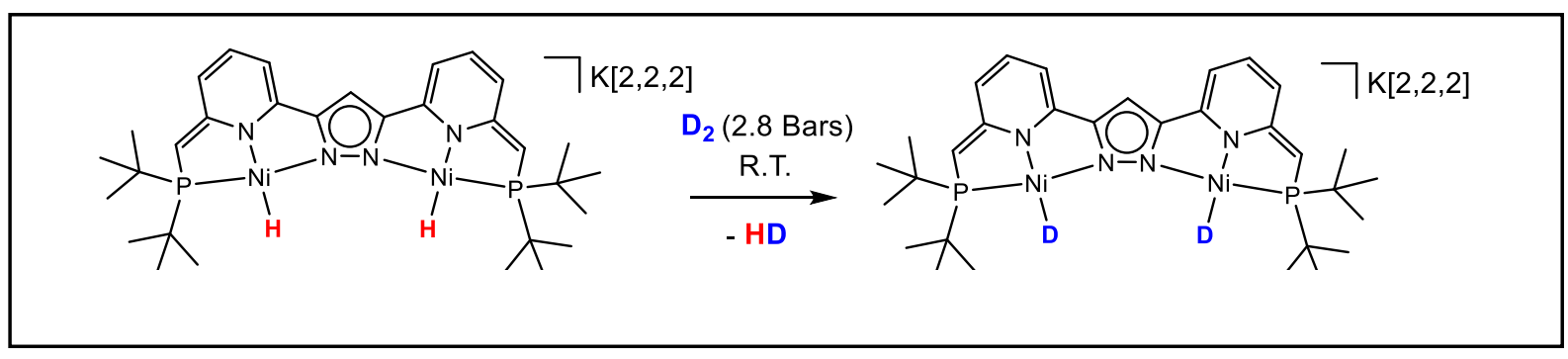

$\mathrm{K}[2,2,2]\left[\mathrm{LNi}_{2}(\mathrm{D})_{2}\right](7): \mathrm{K}[2,2,2]\left[\mathrm{LNi}_{2}(\mathrm{H})_{2}\right](6 \mathrm{mg}, 0.0056 \mathrm{mmol}, 1$ equiv) was dissolved in THF$\mathrm{d}^{8}(0.5 \mathrm{~mL})$ and was transferred in a high pressure Young NMR tube. The tube was degassed by pump freeze thaw cycles. Then, $2.5 \mathrm{~mL}, 2.8$ bar of dried $\mathrm{D}_{2}$ was added. Considering $\mathrm{D}_{2}$ as an ideal gas, calculation with the ideal gas law gave $0.308 \mathrm{mmol}$, which corresponded to 55 equivalents, thus granting a situation where $\mathrm{D}_{2}$ is in excess. $\mathrm{D}_{2}$ was dried under liquid nitrogen according to the set up described below. 


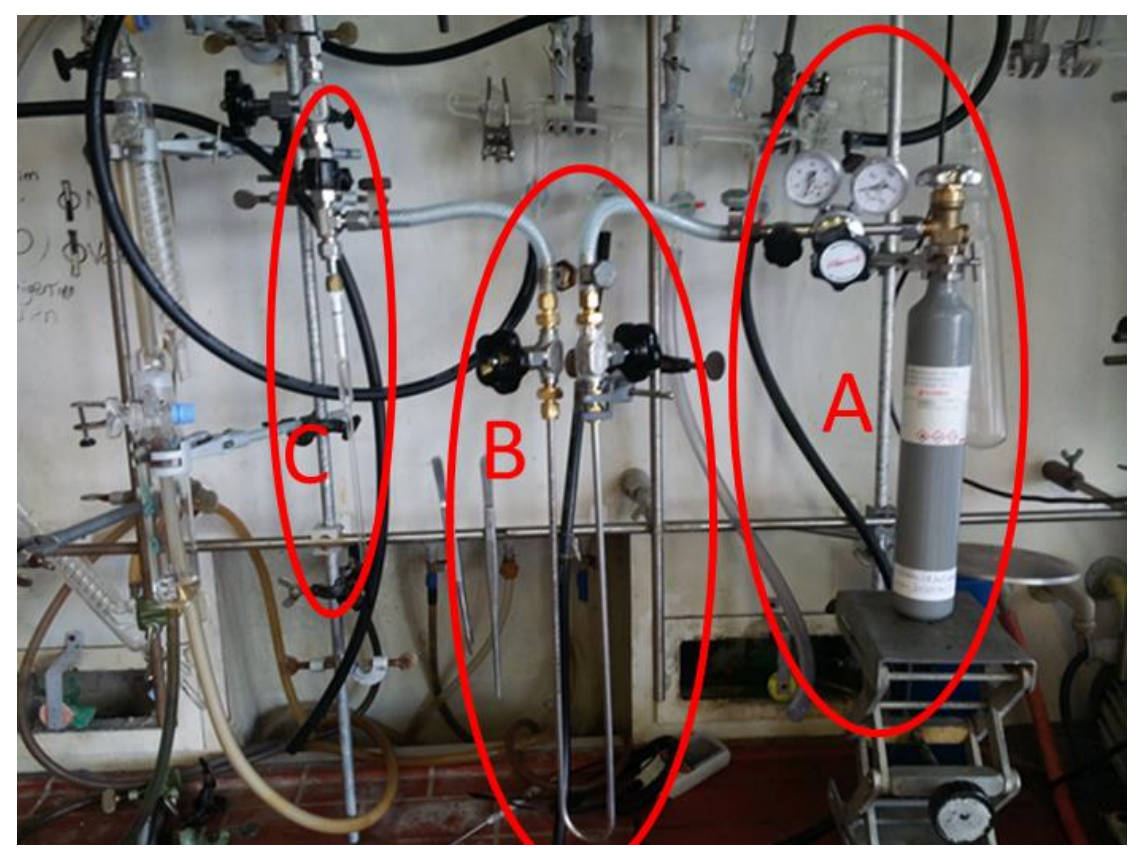

ES12. Red circle $A=$ The $D_{2}$ flask (99.98 \%) with a $180 \mathrm{~mm}$ stainless steel pressure regulator. Red circle $B=$ The " $U$ " pipe in stainless steel with two (in and out) independent manually operating valves. Red circle $C=$ The interface for $D_{2}$ addition screwed to the high-pressure NMR tube and inter-connected with the Schlenk line by a manually operating valve. All A, B and C parts are tightly connected with high pressure plastic pipes. The apparatus is assumed to support at least 15 bars.

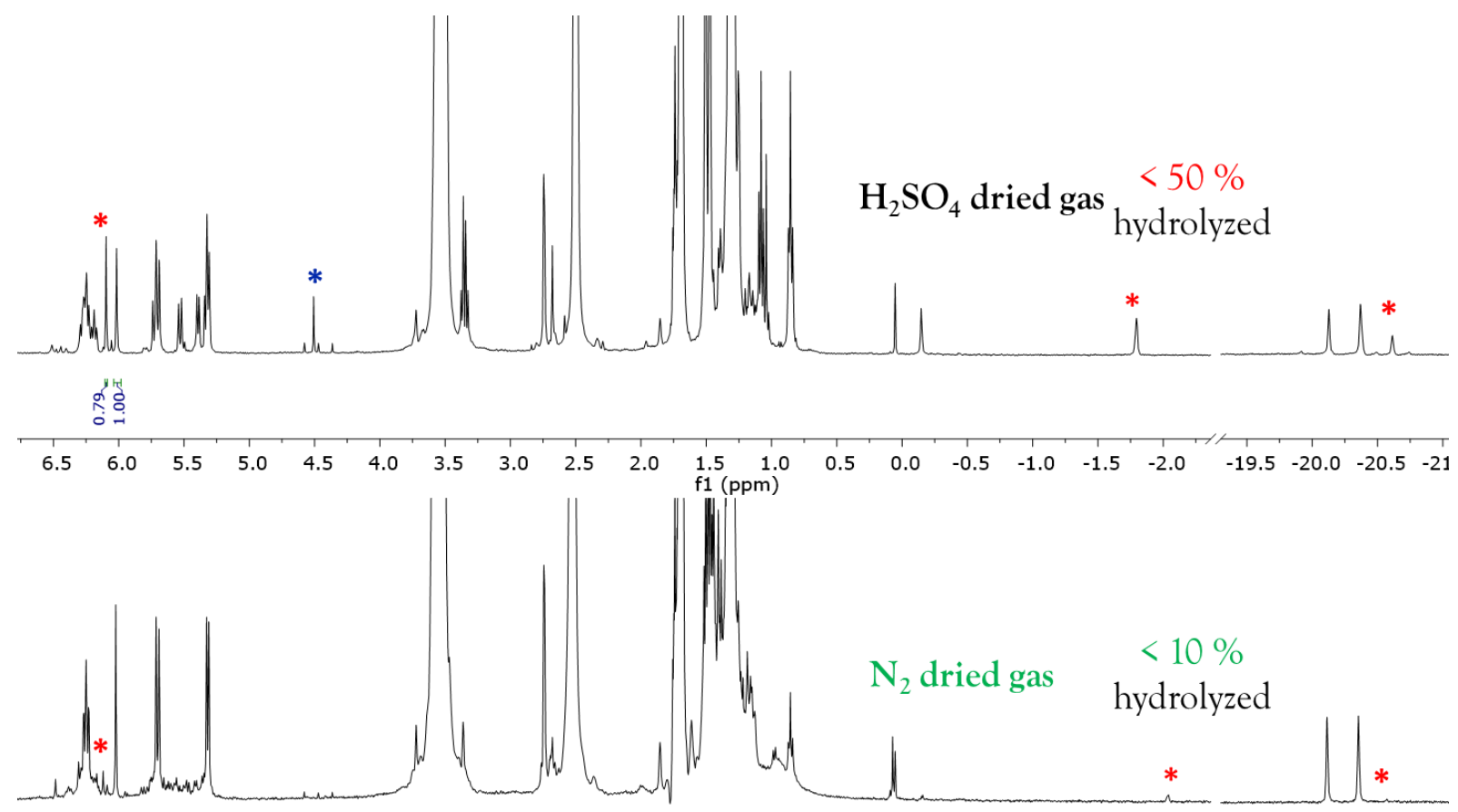

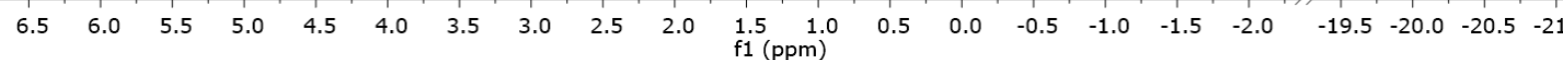

ES13. Different results are obtained after addition of $D_{2}$ according to the drying method used. Using liquid nitrogen to dry $D_{2}$ under pressure with the above-mentioned apparatus was the most adapted method to be employed as the lowest amount of hydrolysed compound $\left({ }^{*}\right)$ was obtained (bottom spectrum). 


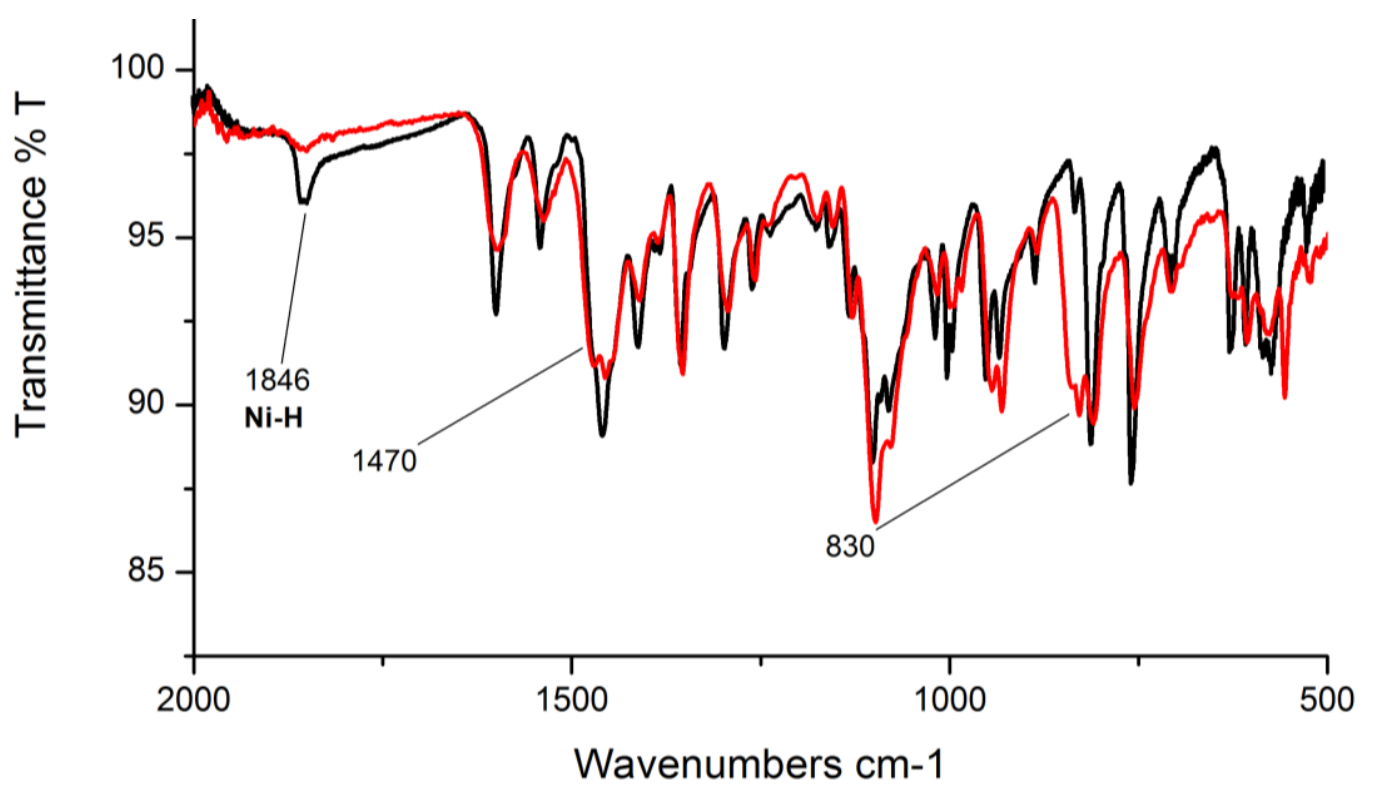

ES14. ATR-IR spectrum of $\mathrm{K}[2,2,2]\left[\mathrm{LNi}_{2}(\mathrm{H})_{2}\right]$ in black and $\mathrm{K}[2,2,2]\left[\mathrm{LNi}_{2}(\mathrm{D})_{2}\right]$ in red. The theoretical value of the Ni-D stretching frequency was calculated according to the equation involving the reduced mass; $\left(v_{\mathrm{Ni}-\mathrm{D}}=v_{\mathrm{Ni}-\mathrm{H}} \sqrt{\frac{\mu_{\mathrm{NiH}}}{\mu_{\mathrm{Ni}-\mathrm{D}}}}=1313 \mathrm{~cm}^{-1}\right)$

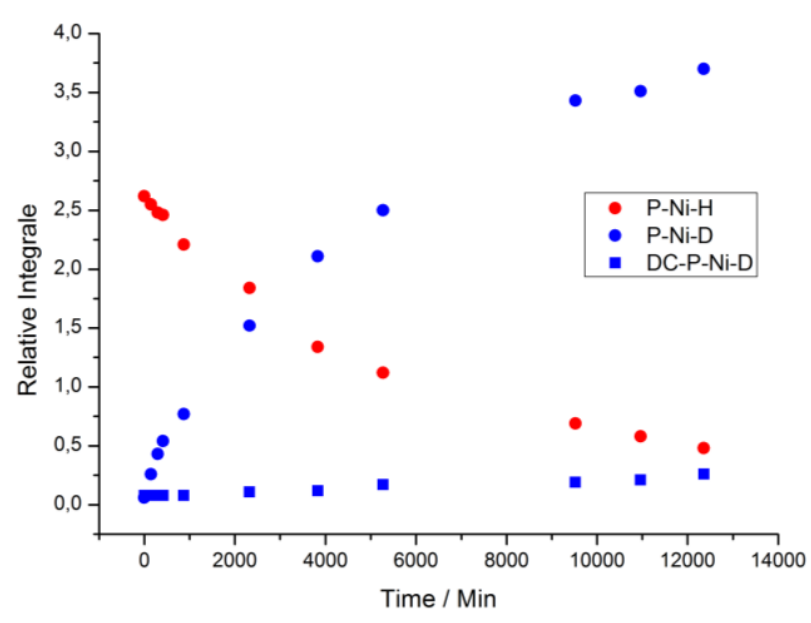

Left Plot: $H / D$ exchange over time. The integrals are studied with ${ }^{31} P$ NMR. The $\mathrm{PF}_{6}$ residual peak at -144 ppm is integrated to 1 as internal reference. The red circles represent the integral of the ${ }^{31} P$ NMR of the P-Ni-H fragment (systematically integrated in a range of $84.07-83.99 \mathrm{ppm})$. The blue circles represent the integral of the ${ }^{31} P$ NMR triplet signal of P-Ni-D (84.44 - 84.21 ppm) and the blue squares represent the integral of the ${ }^{31} P$ NMR signal for DC-P-Ni-D.

ES15. Integrals of the phosphorus signals of $\mathrm{K}[2,2,2]\left[\mathrm{LNi}_{2}(\mathrm{H})_{2}\right]$ plotted against the time during the reaction with $\mathrm{D}_{2}$. 


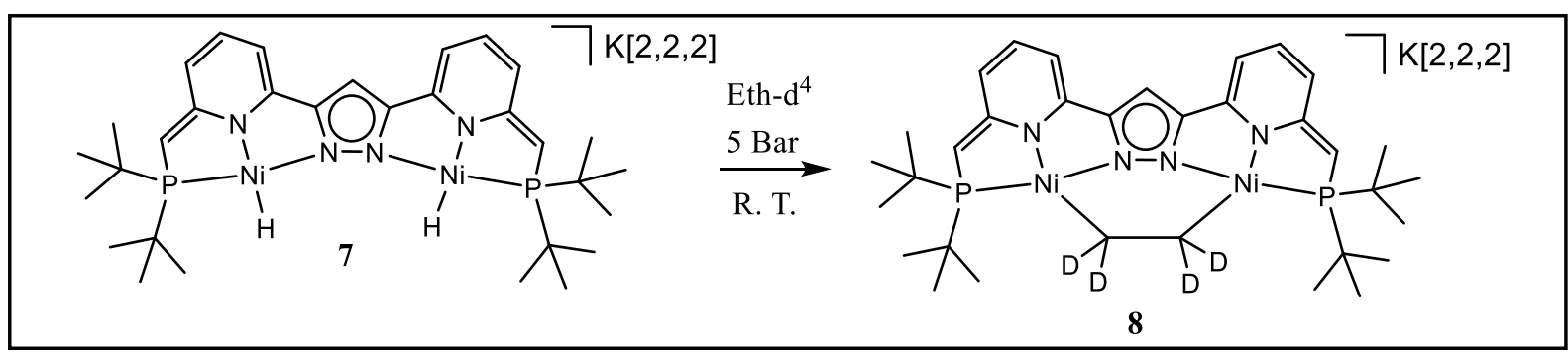

$\mathrm{K}[2,2,2]\left[\mathrm{LNi}\left(\mu-\mathrm{C}_{2} \mathrm{H}_{4}\right) \mathrm{Ni}\right](\mathbf{8}): \mathrm{K}[2,2,2]\left[\mathrm{LNi}_{2}(\mathrm{H})_{2}\right](7)(6 \mathrm{mg}, 0.0056 \mathrm{mmol}, 1$ equiv) was dissolved in THF- $\mathrm{d}^{8}(0.5 \mathrm{~mL})$ and was transferred in a high pressure Young NMR tube. The tube was degassed by pump freeze thaw cycles. Then, $2.5 \mathrm{~mL}$, 5 bars of dried ethylene was added. Ethylene was dried according to the same procedure as for $\mathrm{D}_{2} / \mathrm{H}_{2}$ except the cooling trap was filled with $\mathrm{EtOH}$ and cooled until freezing point. After heating the sample at $308 \mathrm{~K}$ overnight, complex 8 was crystalized by diffusion in dry hexanes.

Elemental formula: $\mathrm{C}_{51} \mathrm{H}_{81} \mathrm{D}_{4} \mathrm{KN}_{6} \mathrm{Ni}_{2} \mathrm{O}_{6} \mathrm{P}_{2}$

Molecular weight: 1100.73 g. $\mathrm{mol}^{-1}$

${ }^{1} \mathrm{H}$ NMR $\left(500 \mathrm{MHz}, \mathrm{THF}^{8}{ }^{8}\right): 6.31\left(\mathrm{dd},{ }^{3} \mathrm{~J}=6.7 \mathrm{~Hz},{ }^{3} \mathrm{~J}=8.6 \mathrm{~Hz}, 2 \mathrm{H}\right), 6.19(\mathrm{~s}, 1 \mathrm{H}) 5.77\left(\mathrm{~d},{ }^{3} \mathrm{~J}=\right.$ $8.6 \mathrm{~Hz}, 2 \mathrm{H}) 5.45\left(\mathrm{~d},{ }^{3} \mathrm{~J}=6.7 \mathrm{~Hz}, 2 \mathrm{H}\right) 3.60(\mathrm{bs}, 12 \mathrm{H}) 3.51(\mathrm{bs}, 12 \mathrm{H}) 2.82(\mathrm{~s}, 2 \mathrm{H}) 2.50(\mathrm{~m}, 12 \mathrm{H})$ $1.37\left(\mathrm{~d},{ }^{3} \mathrm{~J}=12.3 \mathrm{~Hz}, 36 \mathrm{H}\right)$

${ }^{2} \mathrm{H}$ NMR (76.6 MHz, THF-d $\left.{ }^{8}\right): 0.89(\mathrm{br})$

${ }^{13} \mathrm{C}$ NMR (126 MHz, THF-d $\left.{ }^{8}\right): 168.35(\mathrm{~d}, 18.63 \mathrm{~Hz}) 154.93(\mathrm{~d}, 2.72 \mathrm{~Hz}) 149.54(\mathrm{~d}, 3.33 \mathrm{~Hz})$ $132.73(\mathrm{~s}) 111.23(\mathrm{~d}, 14.45 \mathrm{~Hz}) 95.04(\mathrm{~s}) 93.67$ (s) $59.16(\mathrm{~d}, 54.88 \mathrm{~Hz})$

${ }^{31}$ P NMR (202 MHz, THF d $): 56.97$

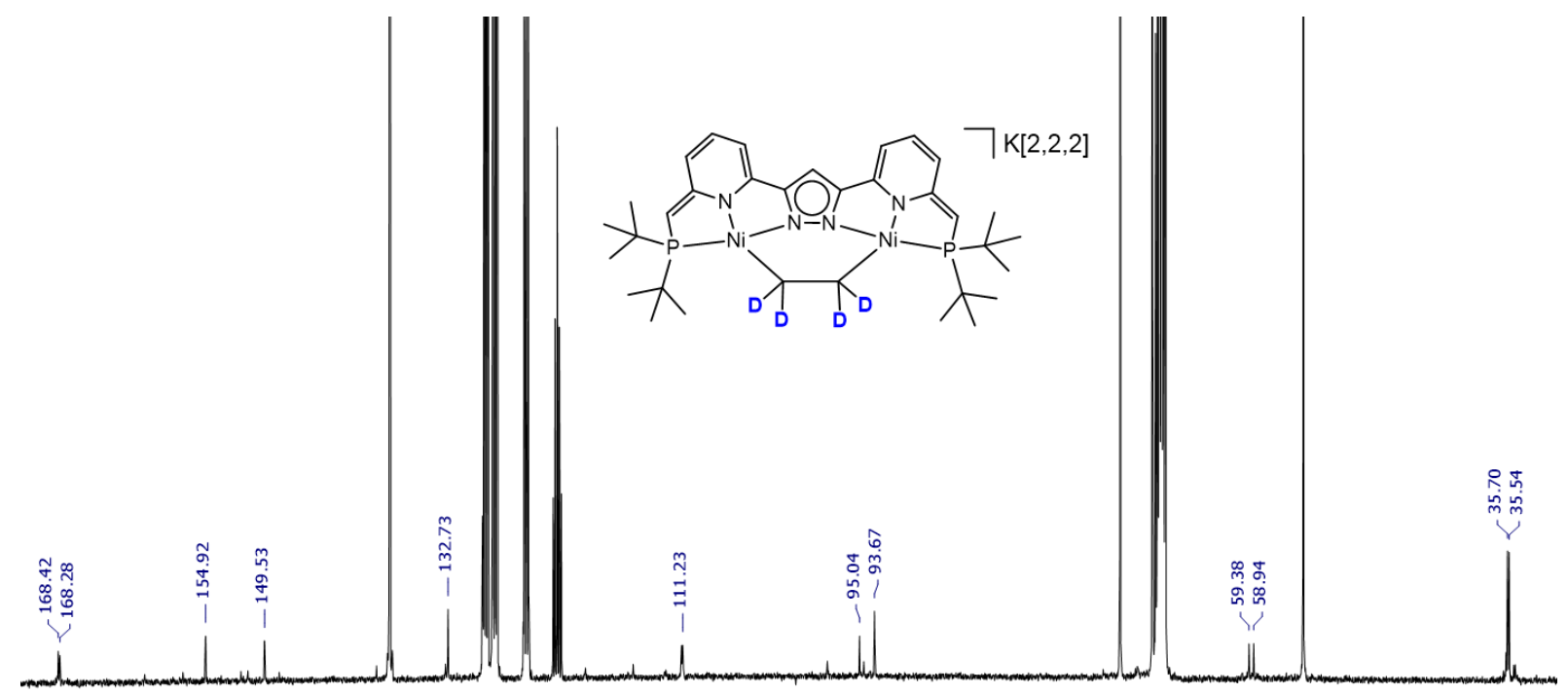

$\begin{array}{lllllllllllllllllllllllllllll}170 & 165 & 160 & 155 & 150 & 145 & 140 & 135 & 130 & 125 & 120 & 115 & 110 & \begin{array}{c}105 \\ \mathrm{f} 1(\mathrm{ppm})\end{array} & 100 & 95 & 85 & 80 & 75 & 70 & 65 & 60 & 55 & 50 & 45 & 40 & 35\end{array}$

ES16. ${ }^{13} \mathrm{C}$ NMR spectrum of complex 8 in THF-d ${ }^{8}(125 \mathrm{MHz})$ 


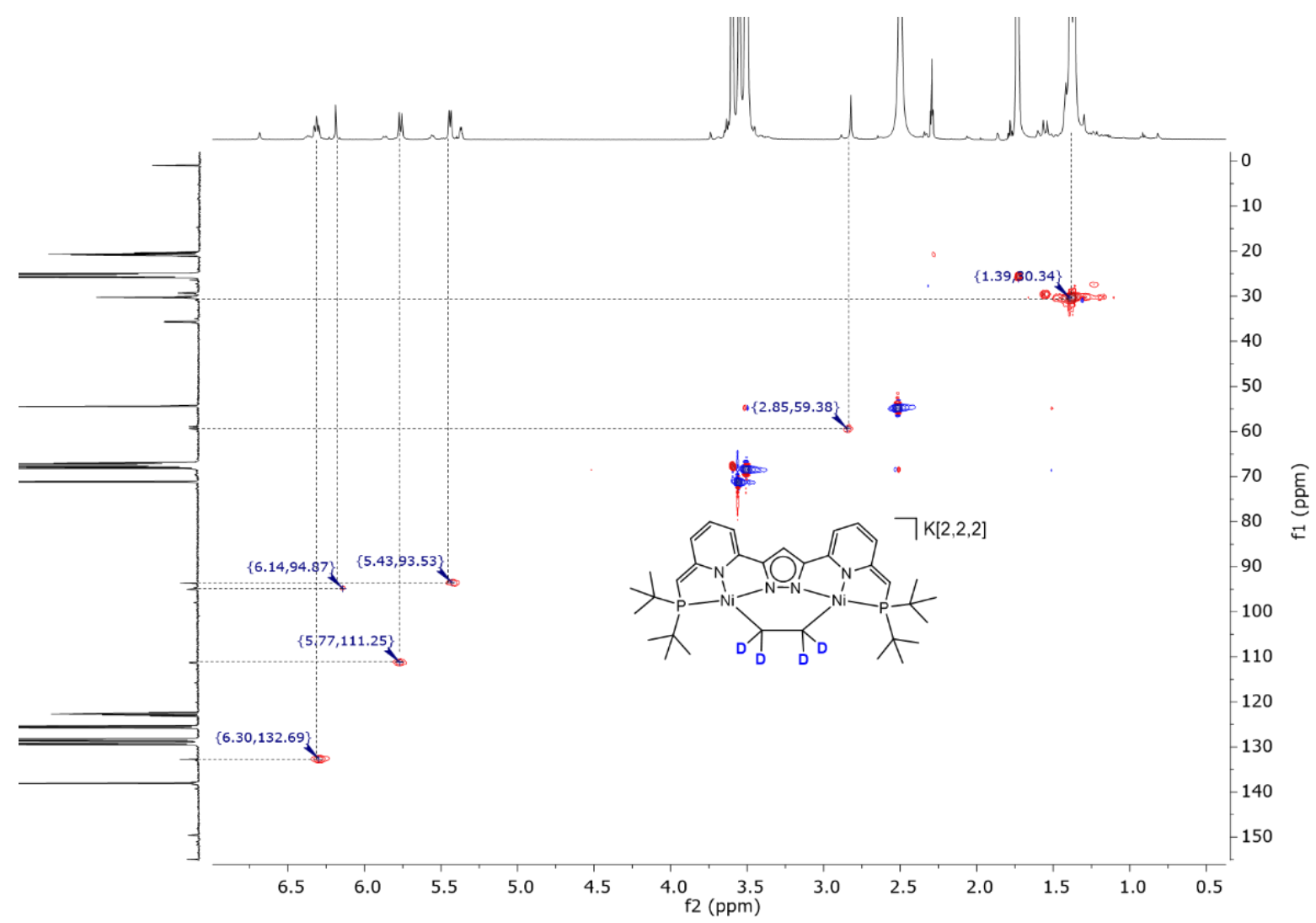

ES17. HSQC spectrum of complex 8 in THF-d ${ }^{8}$

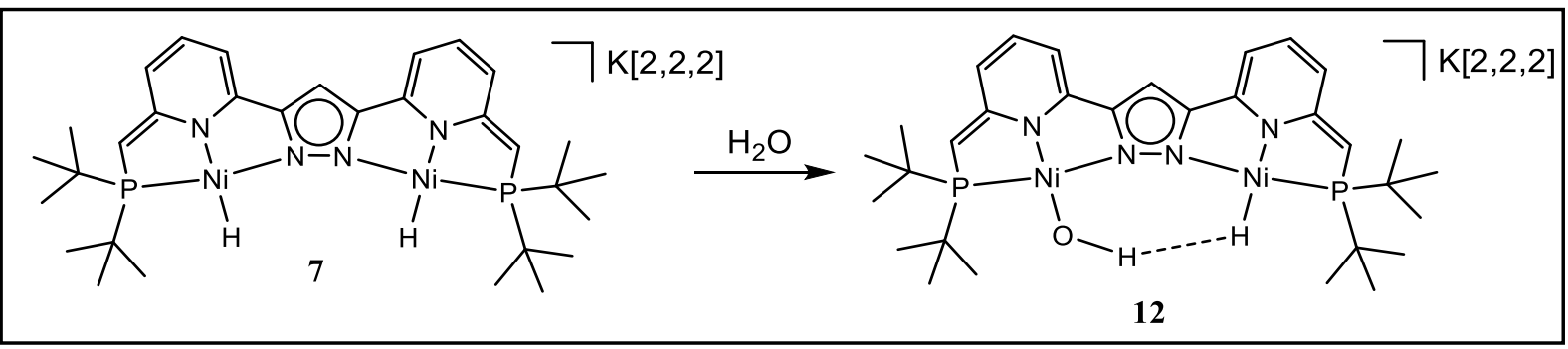

$\mathrm{K}[2,2,2][\mathrm{LNi}(\mathrm{OH}) \mathrm{Ni}(\mathrm{H})](\mathbf{1 2}): \mathrm{K}[2,2,2]\left[\mathrm{LNi}_{2}(\mathrm{H})_{2}\right](\mathbf{7})(10 \mathrm{mg}, 0.0093 \mathrm{mmol}, 1$ equiv), was dissolved in $0.5 \mathrm{~mL}$ of THF- $\mathrm{d}^{8}$ and the solution was transferred in a Young NMR tube. Then a $0.1 \mathrm{M}$ solution of $\mathrm{H}_{2} \mathrm{O}$ in THF-d ${ }^{8}$ was added at room temperature with a Hamilton syringe (94 $\mu L, 1$ eq). The NMR Young tube was closed and shaken. After NMR characterization, it was possible to crystalize complex 12 by liquid diffusion with hexanes at $-35^{\circ} \mathrm{C}$.

Elemental formula: $\mathrm{C}_{49} \mathrm{H}_{83} \mathrm{KN}_{6} \mathrm{Ni}_{2} \mathrm{O}_{7} \mathrm{P}_{2}$

Molecular weight: $1086.67 \mathrm{~g} \cdot \mathrm{mol}^{-1}$

${ }^{1} \mathrm{H}$ NMR $\left(500 \mathrm{MHz}, \mathrm{THF}^{8} \mathrm{~d}^{8}\right) 6.31\left(\mathrm{ddd},{ }^{3} \mathrm{~J}=6.63 \mathrm{~Hz},{ }^{3} \mathrm{~J}=8.71 \mathrm{~Hz},{ }^{5} \mathrm{~J}_{\mathrm{P}-\mathrm{H}}=1.90 \mathrm{~Hz}, 1 \mathrm{H}\right) 6.22(\mathrm{ddd}$, $\left.{ }^{5} \mathrm{~J}_{\mathrm{P} \cdot \mathrm{H}}=1.90 \mathrm{~Hz},{ }^{3} \mathrm{~J}=6.68 \mathrm{~Hz},{ }^{3} \mathrm{~J}=8.60 \mathrm{~Hz}, 1 \mathrm{H}\right) 6.13(\mathrm{~s}, 1 \mathrm{H}) 5.76\left(\mathrm{~d},{ }^{3} \mathrm{~J}=8.71 \mathrm{~Hz}, 2 \mathrm{H}\right) 5.56\left(\mathrm{~d},{ }^{3} \mathrm{~J}\right.$ $=8.75 \mathrm{~Hz}, 2 \mathrm{H}) 5.42\left(\mathrm{~d},{ }^{3} \mathrm{~J}=6.66 \mathrm{~Hz}, 2 \mathrm{H}\right) 5.76\left(\mathrm{dd},{ }^{3} \mathrm{~J}=6.57 \mathrm{~Hz},{ }^{4} \mathrm{~J}=0.82 \mathrm{~Hz}, 2 \mathrm{H}\right) 3.60(\mathrm{bs}, 12$ H) $3.55(\mathrm{~m}, 12 \mathrm{H}) 2.77\left(\mathrm{~d},{ }^{4} \mathrm{~J}_{\mathrm{H}-\mathrm{H}}=2.29 \mathrm{~Hz}, 1 \mathrm{H}\right) 2.70(\mathrm{~s}, 1 \mathrm{H}) 2.55(\mathrm{~m}, 12 \mathrm{H}) 1.52\left(\mathrm{~d},{ }^{3} \mathrm{~J}=12.90\right.$ $\mathrm{Hz}, 18 \mathrm{H}) 1.33\left(\mathrm{~d},{ }^{3} \mathrm{~J}=12.90 \mathrm{~Hz}, 18 \mathrm{H}\right)-1.78(\mathrm{~s}, 1 \mathrm{H})-20.46\left(\mathrm{dd},{ }^{2} \mathrm{~J}_{\mathrm{P} \cdot \mathrm{H}}=93.11 \mathrm{~Hz},{ }^{4} \mathrm{~J}_{\mathrm{H} \cdot \mathrm{H}}=2.29 \mathrm{~Hz}\right.$, $1 \mathrm{H})$. 
${ }^{13} \mathrm{C}$ NMR (126 MHz, THF-d $\left.{ }^{8}\right) 170.05\left(\mathrm{~d},{ }^{2} \mathrm{~J}=18.34 \mathrm{~Hz}\right) 168.32\left(\mathrm{~d},{ }^{2} \mathrm{~J}=18.81 \mathrm{~Hz}\right) 157.49\left(\mathrm{~d},{ }^{3} \mathrm{~J}=\right.$ $3.50 \mathrm{~Hz}) 154.42\left(\mathrm{~d},{ }^{3} \mathrm{~J}=2.98 \mathrm{~Hz}\right) 150.18\left(\mathrm{~d},{ }^{3} \mathrm{~J}=2.93 \mathrm{~Hz}\right) 150.00\left(\mathrm{~d},{ }^{3} \mathrm{~J}=2.64 \mathrm{~Hz}\right) 133.25\left(\mathrm{~d},{ }^{4} \mathrm{~J}=\right.$ $2.04 \mathrm{~Hz}) 132.03\left(\mathrm{~d},{ }^{4} \mathrm{~J}=1.93 \mathrm{~Hz}\right) 111.25\left(\mathrm{~d},{ }^{3} \mathrm{~J}=16 \mathrm{~Hz}\right) 110.51\left(\mathrm{~d},{ }^{3} \mathrm{~J}=16 \mathrm{~Hz}\right) 95.26(\mathrm{~s}) 95.19(\mathrm{~s})$ $94.28(\mathrm{~s}) 58.83\left(\mathrm{~d},{ }^{1} \mathrm{~J}=53.00 \mathrm{~Hz}\right) 57.57\left(\mathrm{~d},{ }^{1} \mathrm{~J}=56.65 \mathrm{~Hz}\right) 35.72\left(\mathrm{~d},{ }^{1} \mathrm{~J}=21.00 \mathrm{~Hz}\right) 34.76\left(\mathrm{~d},{ }^{1} \mathrm{~J}=\right.$ $26.32 \mathrm{~Hz}) 30.00\left(\mathrm{~d},{ }^{2} \mathrm{~J}=4.54 \mathrm{~Hz}\right) 29.47\left(\mathrm{~d},{ }^{2} \mathrm{~J}=3.95 \mathrm{~Hz}\right)$.

${ }^{31}$ P NMR (202 MHz, THF d $\left.{ }^{8}\right) 85.16,50.56$.

MS-ESI (-), THF m/z: 669.2: [LNi(OH)Ni(H)]-

IR (ATR) v/ $\mathrm{cm}^{-1}: 3458(\mathrm{NiO}-\mathrm{H}) 1854(\mathrm{Ni}-\mathrm{H})$.
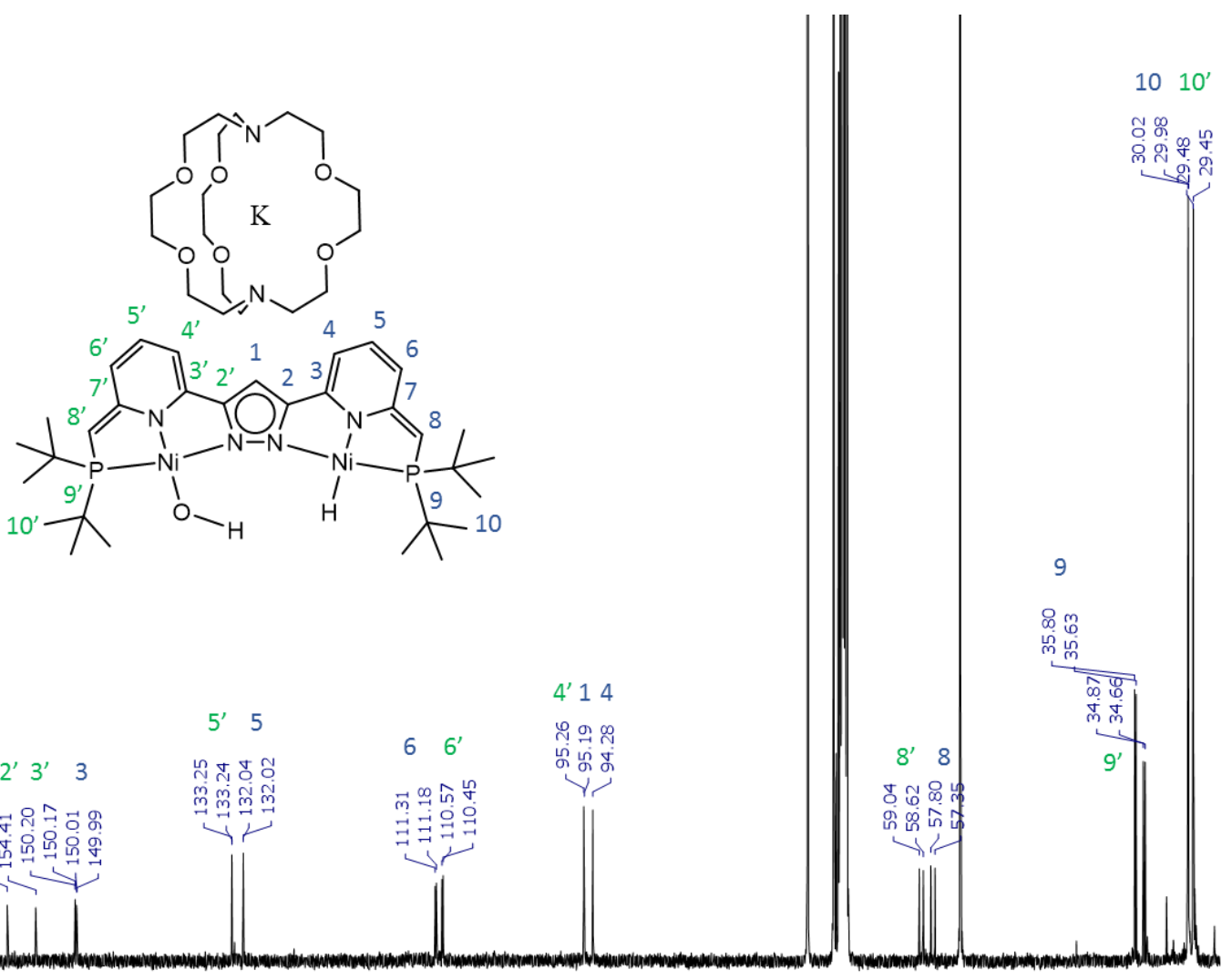

170
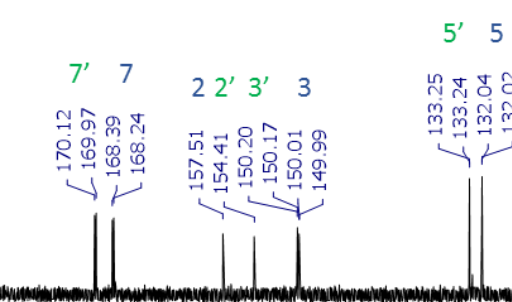

ํํㄴㅎํㅇ

m m m

$\stackrel{m}{\stackrel{m}{m} \underset{-}{m}}$

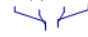




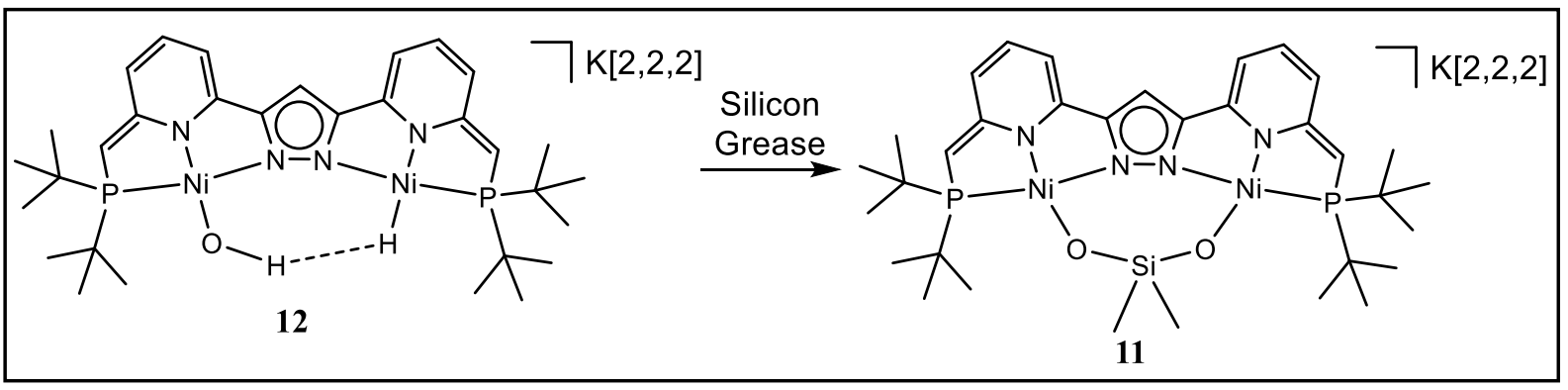

$\mathrm{K}[2,2,2]\left[\mathrm{LNi}\left(\mu-\mathrm{O}_{2} \mathrm{SiMe}_{2}\right) \mathrm{Ni}\right](\mathbf{1 1}): \mathrm{K}[2,2,2][\mathrm{LNi}(\mathrm{OH}) \mathrm{Ni}(\mathrm{H})]$ quantitatively reacted with traces of grease in the NMR sample. Once the compound formed it crystallized from liquid diffusion with hexanes at $-35^{\circ} \mathrm{C}$.

Elemental formula: $\mathrm{C}_{51} \mathrm{H}_{89} \mathrm{KN}_{6} \mathrm{Ni}_{2} \mathrm{O}_{9} \mathrm{P}_{2} \mathrm{Si}$

Molecular weight: $1176.82 \mathrm{~g} \cdot \mathrm{mol}^{-1}$

${ }^{1} \mathrm{H}$ NMR $\left(500 \mathrm{MHz}, \mathrm{THF}^{8}{ }^{8}\right): 6.27\left(\mathrm{ddd},{ }^{5} \mathrm{~J}_{\mathrm{P}-\mathrm{H}}=1.15 \mathrm{~Hz},{ }^{3} \mathrm{~J}=6.62 \mathrm{~Hz},{ }^{3} \mathrm{~J}=8.64 \mathrm{~Hz}, 2 \mathrm{H}\right), 6.17(\mathrm{~s}$, 1H) $5.60\left(\mathrm{~d},{ }^{3} \mathrm{~J}=8.64 \mathrm{~Hz}, 2 \mathrm{H}\right) 5.42\left(\mathrm{~d},{ }^{3} \mathrm{~J}=6.62 \mathrm{~Hz}, 2 \mathrm{H}\right) 3.61(\mathrm{bs}, 12 \mathrm{H}) 3.56(\mathrm{~m}, 12 \mathrm{H}) 2.65(\mathrm{~s}$, 2H) $2.56(\mathrm{~m}, 12 \mathrm{H}) 1.51\left(\mathrm{~d},{ }^{3} \mathrm{~J}=13.11 \mathrm{~Hz}, 36 \mathrm{H}\right) 0.06(\mathrm{br}, 6 \mathrm{H})$.

${ }^{13} \mathrm{C}$ NMR (126 MHz, THF-d $\left.{ }^{8}\right): 170.44\left(\mathrm{~d},{ }^{2} \mathrm{~J}=17.96 \mathrm{~Hz}\right) 154.87\left(\mathrm{~d},{ }^{3} \mathrm{~J}=2.34 \mathrm{~Hz}\right) 151.01\left(\mathrm{~d},{ }^{4} \mathrm{~J}=\right.$ $2.36 \mathrm{~Hz}) 132.79$ (s) $111.71\left(\mathrm{~d},{ }^{3} \mathrm{~J}=16.38 \mathrm{~Hz}\right) 97.03$ (s) 95.40 (s) $58.38\left(\mathrm{~d},{ }^{1} \mathrm{~J}=57.93 \mathrm{~Hz}\right) 36.24$ $\left(\mathrm{d},{ }^{1} \mathrm{~J}=21.70 \mathrm{~Hz}\right) 29.38\left(\mathrm{~d},{ }^{2} \mathrm{~J}=3.33 \mathrm{~Hz}\right) 5.43(\mathrm{br})$.

${ }^{31}$ P NMR: $\left(202 \mathrm{MHz}, \mathrm{THF}^{8}{ }^{8}\right): 49.52$.
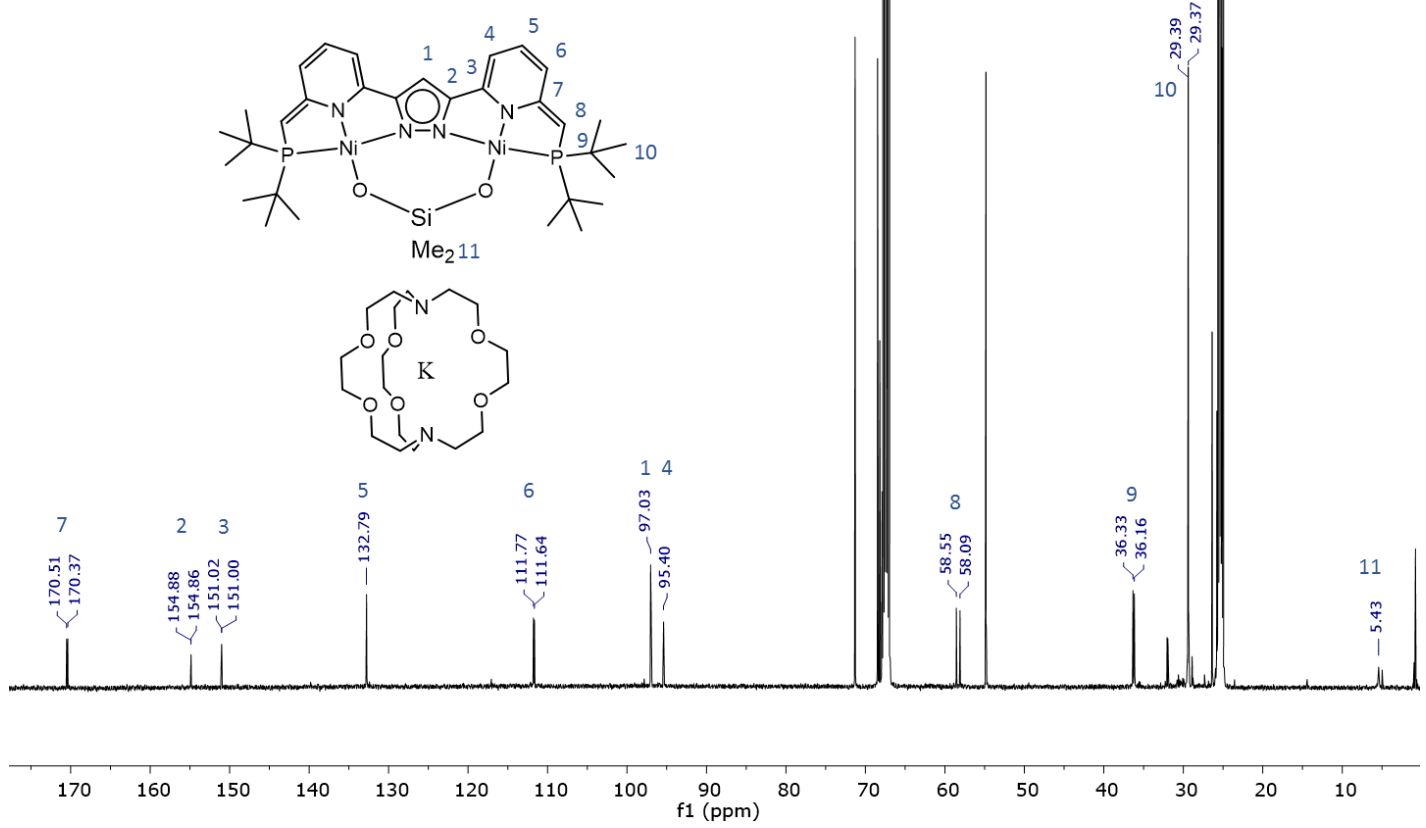

ES19. ${ }^{13} \mathrm{C}$ NMR of complex 11 in THF-d ${ }^{8}(126 \mathrm{MHz})$ 


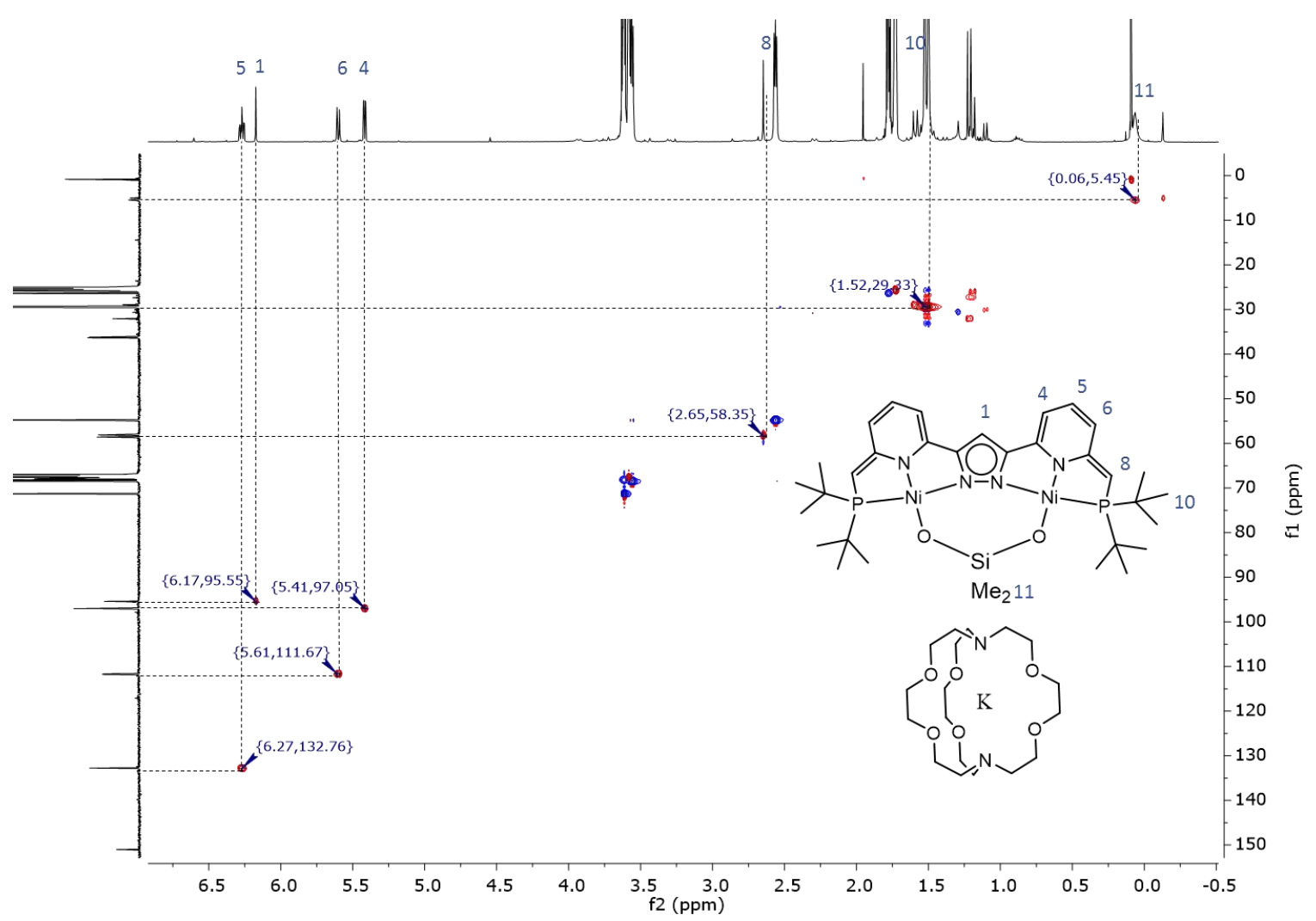

ES20. ${ }^{13} \mathrm{C}$ NMR of complex 11 in THF-d ${ }^{8}(126 \mathrm{MHz})$ 


\begin{tabular}{|c|c|c|c|}
\hline Compound & Complex 1 & Complex 2 & Complex 3 \\
\hline empirical formula & $\mathrm{C}_{47} \mathrm{H}_{74} \mathrm{~F}_{6} \mathrm{~N}_{5} \mathrm{Ni}_{2} \mathrm{O}_{5} \mathrm{P}_{3}$ & $\mathrm{C}_{31} \mathrm{H}_{47} \mathrm{Br}_{2} \mathrm{~F}_{6} \mathrm{~N}_{4} \mathrm{Ni}_{2} \mathrm{O}_{0.50} \mathrm{P}_{3}$ & $\mathrm{C}_{34} \mathrm{H}_{50} \mathrm{Br}_{2} \mathrm{~F}_{3} \mathrm{FeN}_{5} \mathrm{NiO}_{3} \mathrm{P}_{2} \mathrm{~S}$ \\
\hline formula weight & 1113.44 & 967.87 & 1002.17 \\
\hline $\mathrm{T} / \mathrm{K}$ & $133(2)$ & $133(2)$ & $133(2)$ \\
\hline crystal size $/ \mathrm{mm}^{3}$ & $0.50 \times 0.50 \times 0.37$ & $0.50 \times 0.20 \times 0.16$ & $0.34 \times 0.17 \times 0.15$ \\
\hline crystal system & Triclinic & Triclinic & Monoclinic \\
\hline space group & $\mathrm{P}-1$ & $\mathrm{P}-1$ & $\mathrm{P} 2_{1} / n$ \\
\hline $\mathrm{a} / \AA$ & $14.7480(4)$ & $8.5808(4)$ & $13.7239(4)$ \\
\hline $\mathrm{b} / \AA$ & $15.9613(5)$ & $15.0453(8)$ & $13.3856(3)$ \\
\hline$c / \AA$ & $24.8003(7)$ & $15.4575(8)$ & $23.3029(7)$ \\
\hline $\mathrm{a} /{ }^{\circ}$ & $81.127(2)$ & $104.084(4)$ & 90 \\
\hline $\mathrm{b} /{ }^{\circ}$ & $73.850(2)$ & $96.041(4)$ & $102.793(2)$ \\
\hline $\mathrm{g} /{ }^{\circ}$ & $79.470(2)$ & $95.082(4)$ & 90 \\
\hline$V / \AA^{3}$ & $5480.0(3)$ & 1911.30(17) & $4174.5(2)$ \\
\hline $\mathrm{Z}$ & 4 & 2 & 4 \\
\hline$\rho / \mathrm{g} \mathrm{cm}^{-3}$ & 1.350 & 1.682 & 1.595 \\
\hline$F(000)$ & 2344 & 980 & 2040 \\
\hline$\mu / \mathrm{mm}^{-1}$ & 0.841 & 3.261 & 2.892 \\
\hline $\mathrm{T}_{\min } \mid \mathrm{T}_{\max }$ & $0.6762 \mid 0.7817$ & $0.2828 \mid 0.6716$ & \begin{tabular}{ll|l}
0.5112 & 0.6701 \\
\end{tabular} \\
\hline$\Theta$-range /。 & 1.305 to 25.675 & 1.370 to 26.810 & 1.586 to 25.664 \\
\hline hkl-range & $-17 \leq \mathrm{h} \leq 17$ & $-9 \leq h \leq 10$ & $-16 \leq \mathrm{h} \leq 16$ \\
\hline & $-19 \leq \mathrm{k} \leq 19$ & $-19 \leq \mathrm{k} \leq 19$ & $-16 \leq \mathrm{k} \leq 16$ \\
\hline & $-30 \leq 1 \leq 30$ & $-19 \leq 1 \leq 19$ & $-27 \leq 1 \leq 28$ \\
\hline measured refl. & 68257 & 24621 & 46717 \\
\hline unique refl. $/ \mathrm{R}_{\text {int }}$ & $20663[0.0494]$ & 8089 [0.0578] & $7876[0.0596]$ \\
\hline completeness to $\Theta / \%$ & 100.0 & 99.9 & 100.0 \\
\hline \begin{tabular}{l|l|l} 
data & res. I param.
\end{tabular} & $20663|554| 1481$ & $8089|134| 476$ & $7876|0| 482$ \\
\hline goodness-of-fit $\left(\mathrm{F}^{2}\right)$ & 0.941 & 1.040 & 1.096 \\
\hline $\mathrm{R} 1, \omega \mathrm{R} 2(\mathrm{I}>2 \sigma(\mathrm{I}))$ & $0.0374,0.0814$ & $0.0390,0.0966$ & $0.0545,0.1232$ \\
\hline $\mathrm{R} 1, \omega \mathrm{R} 2$ (all data) & $0.0586,0.0865$ & $0.0484,0.1006$ & $0.0740,0.1306$ \\
\hline resid. el. dens. / e $\AA^{-3}$ & \begin{tabular}{l|l|l|}
0.624 & -0.248 \\
\end{tabular} & $\begin{array}{l}0.966 \mid-0.741 \\
\end{array}$ & $0.944 \mid-1592$ \\
\hline
\end{tabular}




\begin{tabular}{|c|c|c|c|}
\hline Compound & Complex 4 & Complex 7 & Complex 8 \\
\hline empirical formula & $\mathrm{C}_{43} \mathrm{H}_{65} \mathrm{~F}_{18} \mathrm{~N}_{10} \mathrm{Ni}_{2} \mathrm{P}_{5}$ & $\mathrm{C}_{65} \mathrm{H}_{115} \mathrm{KN}_{6} \mathrm{Ni}_{2} \mathrm{O}_{10} \mathrm{P}_{2}$ & $\mathrm{C}_{67} \mathrm{H}_{117} \mathrm{KN}_{6} \mathrm{Ni}_{2} \mathrm{O}_{10} \mathrm{P}_{2}$ \\
\hline formula weight & 1336.32 & 1359.08 & 1385.12 \\
\hline $\mathrm{T} / \mathrm{K}$ & $133(2)$ & $133(2)$ & $133(2)$ \\
\hline crystal size $/ \mathrm{mm}^{3}$ & $0.50 \times 0.44 \times 0.42$ & $0.50 \times 0.17 \times 0.16$ & $0.50 \times 0.39 \times 0.27$ \\
\hline crystal system & Monoclinic & Monoclinic & Monoclinic \\
\hline space group & $\mathrm{Cc}$ & $P 2_{1}$ & $P 2_{1} / \mathrm{c}$ \\
\hline $\mathrm{a} / \AA$ & $25.8764(14)$ & $12.5395(4)$ & $14.3241(2)$ \\
\hline $\mathrm{b} / \AA$ & $12.8728(3)$ & $20.1995(4)$ & $20.0420(2)$ \\
\hline$c / \AA$ & $21.6592(10)$ & $14.5653(4)$ & $24.9119(4)$ \\
\hline$\alpha /{ }^{\circ}$ & 90 & 90 & 90 \\
\hline$\beta /{ }^{\circ}$ & $125.024(3)$ & 105.149 & $94.0630(10)$ \\
\hline$\gamma /{ }^{\circ}$ & 90 & 90 & 90 \\
\hline $\mathrm{V} / \AA^{3}$ & $5908.2(5)$ & $3561.06(17)$ & 7133.82 \\
\hline $\mathrm{Z}$ & 4 & 2 & 4 \\
\hline$\rho / \mathrm{g} \mathrm{cm}^{-3}$ & 1.502 & 1.267 & 1.290 \\
\hline$F(000)$ & 2744 & 1464 & 2984 \\
\hline$\mu / \mathrm{mm}^{-1}$ & 0.870 & 0.689 & 0.689 \\
\hline \begin{tabular}{l|l}
$T_{\min }$ & $T_{\max }$ \\
\end{tabular} & $0.6292 \mid 0.7585$ & $0.6131 \mid 0.8638$ & $0.7067 \mid 0.9070$ \\
\hline$\Theta$-range / 0 & 1.851 to 27.243 & 1.448 to 26.747 & 1.305 to 25.646 \\
\hline \multirow[t]{3}{*}{ hkl-range } & $-33 \leq h \leq 33$ & $-15 \leq \mathrm{h} \leq 15$ & $-17 \leq \mathrm{h} \leq 17$ \\
\hline & $-16 \leq \mathrm{k} \leq 16$ & $-24 \leq \mathrm{k} \leq 25$ & $-22 \leq \mathrm{k} \leq 24$ \\
\hline & $-27 \leq 1 \leq 27$ & $-18 \leq 1 \leq 18$ & $-30 \leq 1 \leq 30$ \\
\hline measured refl. & 58337 & 41145 & 70880 \\
\hline unique refl. $/ \mathrm{R}_{\text {int }}$ & 58337 & 14411 [0.0569] & $13449[0.0258]$ \\
\hline completeness to $\Theta / \%$ & 100.0 & 100.0 & 100.0 \\
\hline \begin{tabular}{l|l} 
data & res. I param.
\end{tabular} & $58337|191| 786$ & \begin{tabular}{ll|l|l|}
$14411 \mid$ & 151 \\
\end{tabular} & $13449|535| 963$ \\
\hline goodness-of-fit $\left(\mathrm{F}^{2}\right)$ & 1.001 & 1.004 & 1.037 \\
\hline $\mathrm{R} 1, \omega \mathrm{R} 2(\mathrm{I}>2 \sigma(\mathrm{I}))$ & $0.0515,0.1337$ & $0.0464,0.1104$ & $0.0424,0.1104$ \\
\hline $\mathrm{R} 1, \omega \mathrm{R} 2$ (all data) & $0.0564,0.1365$ & $0.0539,0.1136$ & $0.0487,0.1162$ \\
\hline resid. el. dens. / e $\AA^{-3}$ & $0.629 \mid-0.276$ & $0.870 \mid-0.295$ & $0.904-0.602$ \\
\hline
\end{tabular}




\begin{tabular}{|c|c|c|}
\hline Compound & Complex 12 & Complex 11 \\
\hline empirical formula & $\mathrm{C}_{57} \mathrm{H}_{97} \mathrm{KN}_{6} \mathrm{Ni}_{2} \mathrm{O}_{9} \mathrm{P}_{2}$ & $\mathrm{C}_{51} \mathrm{H}_{89} \mathrm{KN}_{6} \mathrm{Ni}_{2} \mathrm{O}_{9} \mathrm{P}_{2} \mathrm{Si}$ \\
\hline formula weight & 1228.86 & 1176.83 \\
\hline $\mathrm{T} / \mathrm{K}$ & $133(2)$ & $133(2)$ \\
\hline crystal size $/ \mathrm{mm}^{3}$ & $0.50 \times 0.41 \times 0.13$ & $0.50 \times 0.16 \times 0.14$ \\
\hline crystal system & Triclinic & Monoclinic \\
\hline space group & $P-1$ & $P 2_{1}$ \\
\hline $\mathrm{a} / \AA$ & $14.4647(6)$ & $15.4775(5)$ \\
\hline $\mathrm{b} / \AA$ & 15.1701(6) & 15.8381(3) \\
\hline$c / \AA$ & $15.4097(8)$ & $15.6062(5)$ \\
\hline$\alpha /{ }^{\circ}$ & $108.659(4)$ & 90 \\
\hline$\beta /{ }^{\circ}$ & 90.903(4) & $111.958(2)$ \\
\hline$\gamma /{ }^{\circ}$ & $91.822(3)$ & 90 \\
\hline$V / \AA^{3}$ & $3200.8(3)$ & $3548.09(18)$ \\
\hline $\mathrm{Z}$ & 2 & 2 \\
\hline$\rho / \mathrm{g} \mathrm{cm}^{-3}$ & 1.275 & 1.102 \\
\hline$F(000)$ & 1316 & 1256 \\
\hline$\mu / \mathrm{mm}^{-1}$ & 0.758 & 0.697 \\
\hline \begin{tabular}{l|l}
$T_{\min }$ & $T_{\max }$ \\
\end{tabular} & $0.6022 \mid 0.8535$ & \begin{tabular}{ll|l|}
0.8398 & 0.9418 \\
\end{tabular} \\
\hline$\Theta$-range / 0 & 1.395 to 26.926 & 1.407 to 26.936 \\
\hline \multirow[t]{3}{*}{ hkl-range } & $-18 \leq \mathrm{h} \leq 18$ & $-19 \leq \mathrm{h} \leq 19$ \\
\hline & $-19 \leq \mathrm{k} \leq 19$ & $-17 \leq \mathrm{k} \leq 20$ \\
\hline & $-19 \leq 1 \leq 19$ & $-19 \leq 1 \leq 19$ \\
\hline measured refl. & 44106 & 44308 \\
\hline unique refl. $/ \mathrm{R}_{\text {int }}$ & $13622[0.0424]$ & $14046[0.0429]$ \\
\hline completeness to $\Theta / \%$ & 100.0 & 100.0 \\
\hline data | res. I param. & $13622|365| 812$ & $14046|3| 669$ \\
\hline goodness-of-fit $\left(\mathrm{F}^{2}\right)$ & 1.027 & 1.007 \\
\hline $\mathrm{R} 1, \omega \mathrm{R} 2(\mathrm{I}>2 \sigma(\mathrm{I}))$ & $0.0437,0.1020$ & $0.0333,0.0661$ \\
\hline $\mathrm{R} 1, \omega \mathrm{R} 2$ (all data) & $0.0745,0.1185$ & $0.0489,0.0713$ \\
\hline resid. el. dens. / e $\AA^{-3}$ & $0.540 \mid-0.352$ & $\begin{array}{l}0.0257 \mid-0.197 \\
\end{array}$ \\
\hline
\end{tabular}




\section{Bibliographie}

${ }^{1}$ J. Klingele, S. Dechert, F. Meyer, Coord. Chem. Rev. 2009, 253, 2698-2741

${ }^{2}$ K. Dalle, F. Meyer, Eur. J. Inorg. Chem. 2015, 3391-3406

${ }^{3}$ J. R. Khusnutdinova, D. Milstein, Angew. Chem. Int. Ed, 2015, 54, 12236-12273

${ }^{4}$ G. Wilke, Angew. Chem. In. Ed. 1988, 27, 185-206

${ }^{5}$ Z. Wang, Comprehensive Organic Name Reactions and Reagents, 2010, 554, 2454-2457

${ }^{6}$ M. Che, Catalysis Today 2013, 218-219, 162-171

${ }^{7}$ V. P. Ananikov, ACS Catal. 2015, 5, 1964-1971

${ }^{8}$ K. Ziegler, E Holzkamp, H. Breil, H. Martin, Angew. Chem. 1955, 67, 541-547

${ }^{9}$ S. Z. Tasker, E. A. Standley, T. F. Jamison, Nature 2014, 509, 299-309

${ }^{10}$ C. J. Moulton, R. S. Shaw, J. Chem. Soc. Dalton Trans. 1976, 0, 1020-1024

${ }^{11}$ J. P. Birk, M. Ronan, I. Bennett, C. Kinney, J. Chem. Educ. 1991, 68, 48-54

${ }^{12}$ G. Maki, J. Chem. Phys. 1958, 29, 1129-1138

${ }^{13}$ G. J. Bullen, R. Mason, P. Pauling, Nature 1961, 189, 291-292

${ }^{14}$ J. P. Fackler, M .L. Mittleman, H. Weigolda, G. M.Barrow, J. Phys. Chem. 1968, 72, 46314636

${ }^{15}$ J. P. Fackler, F. A. Cotton, J. Am. Chem. Soc. 1961, 83, 3775-3778

${ }^{16}$ A. Döhring, R. Goddard, P. W. Jolly, C. Krüger, V. R. Polyakov, Inorg. Chem. 1997, 36, 177 183

${ }^{17}$ F. A. Cotton, J. J. Fackler, Inorg. Chem. 1966, 5, 1200-1207

${ }^{18}$ E. S. Akturk, S. J. Scappaticci, R. N. Seals, M. P. Marshak, Inorg. Chem. 2017, 56, $11466-$ 11469

${ }^{19}$ E. Uhlig, Coord. Chem. Rev. 1973, 10, 227-264

${ }^{20}$ R. G. Hayter, F. S. Humiec, J. Am. Chem. Soc. 1962, 84, 2004-2005

${ }^{21}$ D. L. M. Goodgame, M. Goodgame, F. A. Cotton, J. Am. Chem. Soc., 1961, 83, 4161-4167

${ }^{22}$ R. G. Hayter, and F. S. Humiec, Inorg. Chem. 1965, 4, 1701-1706

${ }^{23}$ Y. Casta, Ph.D. Thesis, University of Neuchâtel, 2008

${ }^{24}$ R. G. Hayter, Inorg. Chem. 1963, 2, 932-935

${ }^{25}$ P. L. Orioli, C. A. Ghilardi, J. Chem. Soc. (A) 1970, 0, 1511-1516

${ }^{26}$ C. Lescot, S. Savourey, P. Thuéry, G. Lefèvre, J. C. Berthet, . T. Cantat, C. R. Chimie 2016, $19,57-70$

${ }^{27}$ D. Benito-Garagorri, K. Mereiter, K. Kirchner, Eur. J. Inorg. Chem 2006, 4374-4379

${ }^{28}$ S. Zhang, R. Pattacini, P. Braunstein, Organometallics 2010, 29, 6660-6667

${ }^{29}$ R. Morassi, I. Bertini, L. Sacconi, Coord. Chem. Rev. 1973, 11, 343-402

${ }^{30}$ L. Sacconi, I. Bertini, J. Am. Chem. Soc. 1968, 90, 5443-5446

${ }^{31}$ I. A. Alfurayj, V. G. Young, M. P. Jensen, Inorg. Chem. 2016, 55, 1469-1479

${ }^{32}$ L. Sacconi, Coord. Chem. Rev. 1972, 8, 351-367

${ }^{33}$ E. K. Barefield, D. H. Bush, S. M. Nelson, Q. Rev. Chem. Soc. 1968, 22, 457-498

${ }^{34}$ S. M. Nelson, W. S. J. Kelly, Chem. Commun. 1968, 436-437

${ }^{35}$ W. S. J. Kelly, G. H. Ford, S. M. Nelson, J. Chem. Soc. (A) 1971, 0, 388-396

${ }^{36}$ H. Ma, J. L. Petersen, V. G. Young, G. T. Yee, M. P. Jensen, J. Am. Chem. Soc. 2011, 133, 5644-5647 
${ }^{37}$ L. Sacconi, M. Morassi, J. Chem. Soc. (A) 1968, 0, 2997-3002

${ }^{38}$ P. Kar, M. Yoshida, Y. Shigeta, A. Usui, A. Kobayashi, T. Minamidate, N. Matsunaga, M. Kato, Angew. Chem. Int. Ed. 2017, 56, 2345-2349

${ }^{39}$ H. Brandenburg, J. Krahmer, K Fischer, B. Schwager, B. Flöser, C. Näther, F. Tuczek. Eur. J. Inorg. Chem. 2018, 576-585

${ }^{40}$ A. J. Bridgeman, Dalton Trans. 2008, 0, 1989-1992.

${ }^{41}$ M. Dommaschk, F. Gutzeit, S. Boretius, R. Haag, R. Herges, Chem. Commun. 2014, 50, $12476-12478$

${ }^{42}$ D. Achey, G. J. Meyer, Inorg. Chem. 2013, 52, 9574-9582

${ }^{43}$ S. Venkataramani,U. Jana, M. Dommaschk,F. D. Sönnichsen,F. Tuczek, R. Herges, Science 2011, 331, 445-448

${ }^{44}$ F. Gutzeit, M. Dommaschk, N. Levin, A. Buchholz, E. Schaub, W. Plass, C. Näther, R. Herges, Inorg. Chem. 2019, DOI: 10.1021/acs.inorgchem.9b00348

${ }^{45}$ G. Leibeling, S. Demeshko, S. Dechert, F. Meyer, Angew. Chem. Int. Ed. 2005, 44, 7111-7114

${ }^{46}$ F. Meyer, P. Kircher, H. Pritzkow, Chem. Commun. 2003, 774-775

${ }^{47}$ S. Demeshko, G. Leibeling, S. Dechert, F. Meyer, Dalton Trans. 2006, 3458-3465

${ }^{48}$ G. Noël, J. C. Röder, S. Dechert, H. Pritzkow, L. Bolk, S. Mecking, F. Meyer, Adv. Synth. Catal. 2006, 348, 887-897

${ }^{49}$ A. Sachse, S. Demeshko, S. Dechert, V. Daebel, A. Lange, F. Meyer, Dalton Trans. 2010, 39, 3903-3914

${ }^{50}$ J. Pons, X. Lopez, E. Benet, J. Casabo, Polyhedron 1990, 9, 2839-2845

${ }^{51}$ T. Simler, L. Karmazin, C. Bailly, P. Braunstein, Organometallics 2016, 35, 903-912

${ }^{52}$ A. Gers-Barlag, Ph.D Thesis, University of Göttingen, 2016, 3, 64

${ }^{53}$ K. Dalle, Ph.D. Thesis, University of Göttingen, 2014, 2, 26

${ }^{54}$ W. Lin, S. R. Wilson, G. S. Girolami, Inorg. Chem. 1997, 36, 2662-2669

${ }^{55}$ G. A. Bain, J. F. Berry, J. Chem. Educ., 2008, 85, 532

${ }^{56}$ P. Roquette, A. Maronna, M. Reinmuth, E. Kaifer, M. Enders, H. J. Himmel, Inorg. Chem. 2011, 50, 1942-1955

${ }^{57}$ A. C. Sander, Ph.D Thesis, University of Göttingen, 2015, 3, 63-65

${ }^{58}$ A. Gers-Barlag, Ph.D Thesis, University of Göttingen, 2016, 3, 94-99

${ }^{59}$ G. R. Fulmer, A. J. M. Miller, N. H. Sherden, H. E. Gottlieb, A. Nudelman, B. M. Stoltz, J.

E. Bercaw, K. I. Goldberg, Organometallics 2010, 29, 2176-2179

${ }^{60}$ J. Novotný, D. Přichystal, M. Sojka, S. Komorovsky, M. Necas, R. Marek, Inorg. Chem. 2018, 57, 641-652

${ }^{61}$ L. Jeremias, J. Novotný, M. Repisky, S. Komorovsky, R. Marek, Inorg. Chem. 2018, 57, 87488759

${ }^{62}$ J. Novotný, M. Sojka, S. Komorovsky, M. Nečas, R. Marek, J. Am. Chem. Soc. 2016, 138, $8432-8445$

${ }^{63}$ M. Damjanovic, K. Katoh, M. Yamashita, M. Enders, J. Am. Chem. Soc. 2013, 135, 1434914358

${ }^{64}$ T. Morita, M. Damjanović, K. Katoh, Y. Kitagawa, N. Yasuda, Y. Lan, W. Wernsdorfer, B. K. B., M. Enders, M. Yamashita, J. Am. Chem. Soc. 2018, 140, 2995-3007

${ }^{65}$ C. Belle, C. Bougault, M. T. Averbuch, A. Durif, J. L. Pierre, J. M. Latour, L. L. Pape, J. Am. Chem. Soc. 2001, 123, 8053-8066.

${ }^{66}$ R. Knoor, H. Hauer, A. Weiss, H. Polzer, F. Ruf, P. Löw, P. Dvortsák, P. Böhrer, Inorg. Chem. $2007,46,8379-8390$ 
${ }^{67}$ M. Ubbink, J. A. R., Worrall, G. W. Canters, E. J. J. Groenen, M. Huber, Annu. Rev. Biophys. Biomol. Struct. 2002, 31, 393-422

${ }^{68}$ H. Grützmacher, Angew. Chem. 2008, 120, 1838-1842

${ }^{69}$ G. van Koten, D. Milstein, Top. Organometallic Chem., 2013, 40, 1-356

${ }^{70}$ E. Peris, R. H. Crabtree, Chem. Soc. Rev. 2018, 47, 1959-1968

${ }^{71}$ M. Ulman, R. H. Grubbs, J. Org. Chem. 1999, 64, 7202-7207

${ }^{72}$ W. L. McClennan, S. A. Rufh, J. A. M. Lummiss, D. E. Fogg, J. Am. Chem. Soc. 2016, 138, $14668-14677$

${ }^{73}$ J. I. van der Vlugt, J. N. H. Reek, Angew. Chem. Int. Ed. 2009, 48, 8832-8846

${ }^{74}$ B. A. Jazdzewski, W. B. Tolman, Coord. Chem. Rev. 2000, 200-202, 633-685

${ }^{75}$ K. Wang, E. I. Stiefel, Science 2001, 291, 106-109

${ }^{76}$ W. H. Harman, J. C. Peters, J. Am. Chem. Soc. 2012, 134, 5080-5082

${ }^{77}$ I. Bertini, P. Dapporto, G. Fallani, L. Sacconi, Inorg. Chem. 1971, 10, 1703-1707

${ }^{78}$ J. Zhang, G. Leitus, Y. B. David, D. Milstein, J. Am. Chem. Soc. 2005, 127, 10840-10841

${ }^{79}$ N. A. Espinosa-Jalapa, A. Kumar, G. Leitus, Y. Diskin-Posner, D. Milstein, J. Am. Chem. Soc. 2017, 139, 11722-11725

${ }^{80}$ J. Zhang, M. Gandelman, D. Herrman, G. Leitus, L. J. W. Shimon, Y. Ben-David, D.

Milstein, Inorg. Chim. Acta. 2009, 359, 1955-1960

${ }^{81}$ D. Srimani, A. Mukherjee, A. F. G. Goldberg, G. Leitus, Y. Diskin-Posner, L. J. W. Shimon, Y. B. David, D. Milstein, Angew. Chem. Int. Ed. 2015, 54, 12357-12360

${ }^{82}$ C. P. Yap, Y. Y. Chong, T. S. Chwee, W. Y. Fan, Dalton Trans. 2018, 47, 8483-8488

${ }^{83}$ E. Kinoshita, K. Arashiba, S. Kuriyama, A. Eizawa, K. Nakajima, Y. Nishibayashi, Eur. J. Inorg. Chem. 2015, 1789-1794

${ }^{84}$ M. Feller, Y. Diskin-Posner, L. J. W. Shimon, E. Ben-Ari, D. Milstein, Organometallics 2012, 31, 4083-4101

${ }^{85}$ M. G. Mazzotta, K. R. Pichaandi, P. E. Fanwick, M. M. Abu-Omar, Angew. Chem. Int. Ed. 2014, 53, 8320-8322

${ }^{86}$ D. Vuzman, E. Poverenov, L. J. W. Shimon, Y. Diskin-Posner, D. Milstein, Organometallics 2008, 27, 2627-2634

${ }^{87}$ M. Vogt, O. R. Wheelaghan, M. A. Iron, G. Leitus, Y. D. Posner, L. J. W. Shimon, Y. B. David, D. Milstein, Organometallics 2013, 32, 300-308

${ }^{88}$ E. Khaskin, M. A. Iron, L. J. W. Shimon, J. Zhang, D. Milstein, J. Am. Chem. Soc. 2010, 132, 8542

${ }^{89}$ S. W. Kohl, L. Weiner, L. Schwartzburd, L. Konstantinovski, L. J. W. Shimon, Y. Ben-David, M. A. Iron, D. Milstein, Science 2009, 324, 74-77

${ }^{90}$ E. Balaraman, E. Khaskin, G. Leitus, D. Milstein, Nat. Chem. 2013, 5, 122-125

${ }^{91}$ C. Gunanathan, Y. Ben-David, D. Milstein, Science 2007, 317, 790-792

92 J. Zhang, G. Leitus, Y. Ben-David, D. Milstein, Angew. Chem. Int. Ed. 2006, 45, 1113-1115

${ }^{93}$ A. Anaby, B. Butschke, Y. Ben-David, L. J. W. Shimon, G. Leitus, M. Feller, D. Milstein, Organometallics 2014, 33, 3716-3726

${ }^{94}$ A. Mukherjee, D. Srimani, Y. Ben-David, D. Milstein, Chem. Cat. Chem. 2017, 9, 559-563

${ }^{95}$ E. Balaraman, C. Gunanathan, J. Zhang, L. J. W. Shimon, D. Milstein, Nat. Chem. 2011, 3, 609-614

${ }^{96}$ C. A. Huff, J. W. Kampf, M. S. Sanford, Organometallics, 2012, 31, 4643-4645

${ }^{97}$ C. Gunanathan, D. Milstein, Acc. Chem. Res. 2011, 44, 588-602

${ }^{98}$ B. Gnanaprakasam, J. Zhang, D. Milstein, Angew. Chem. Int. Ed. 2010, 49, 1468-1471 
${ }^{99}$ S. Qu, Y. Dang, C. Song, M. Wen, K.-W. Huang, Z.-X. Wang, J. Am. Chem. Soc. 2014, 136, 4974-4991.

${ }^{100}$ G. A. Filonenko, E. Cosimi, L. Lefort, M. P. Conley, C. Copéret, M. Lutz, E. J. M. Hensen,

E. A. Pidko, ACS Catal. 2014, 4, 2667-2671.

${ }^{101}$ E. Stepowska, H. Jiang, D. Song, Chem. Commun. 2010, 46, 556.

${ }^{102}$ C. Gunanathan, B. Gnanaprakasam, M. A. Iron, L. J. W. Shimon, D. Milstein, J. Am. Chem. Soc. 2010, 132, 14763-14765.

${ }^{103}$ O. Rivada-Wheelaghan, A. Dauth, G. Leitus, Y. Diskin-Posner, D. Milstein, Inorg. Chem. $2015,54,4526-4538$.

${ }^{104}$ J. I. Van Der Vlugt, M. Lutz, E. A. Pidko, D. Vogt, A. L. Spek, Dalton Trans. 2009, 10161023

${ }^{105}$ D. Oren, Y. Diskin-Posner, L. Avram, M. Feller, D. Milstein, Organometallics 2018, 37, $2217-2221$

${ }^{106}$ D. J. Darensbourg, M. Y . Darensbourg, L. Y. Gob, M. Ludvig, P. Wiegreffe, J. Am. Chem.

Soc. $1987,109,7539-7540$

${ }^{107}$ M. F. Laird, M. Pink, N. P. Tsvetkov, H. Fan, K. G. Caulton, Dalton Trans. 2009, 1283-1285

${ }^{108}$ S. Chakraborty, J. Zhang, J. A. Krause, H. Guan, J. Am. Chem. Soc. 2010, 132, 8872-8873

${ }^{109}$ F. Huang, C. Zhang, J. Jiang, Z. X. Wang, H. Guan, Inorg. Chem. 2011, 50, 3816-3825

${ }^{110}$ F. Schneck, J. Ahrens, M. Finger, A. C. Stückl, C. Würtele, D. Schwarzer, S. Schneider, Nature Commun. 2018, 9, 1161

${ }^{111}$ Q. Liu, L. Wu, R. Jackstell, M. Beller, Nature Commun. 2015, 6, 5933

${ }^{112}$ F. Juliá-Hernández, T. Moragas, J. Cornella, R. Martin, Nature 2017, 545, 84-89

${ }^{113}$ C. Yoo, Y Lee, Chem. Sci. 2017, 8, 600-605

${ }^{114}$ S. Samanta, S. Demesko, S. Dechert, F. Meyer, Angew. Chem. Int. Ed. 2015, 54, 583-587

115 A. Gers-Barlag, P. Goursot, S. Dechert, F. Meyer, Mansucript submitted.

${ }^{116}$ K. Sone, Y. Fukuda, Rev. Inorg. Chem. 1990, 11, 123-154

${ }^{117}$ C. T. Chen, S. Y. Liao, K. J. Lin, C. H. Chen, T. Y. J. Lin, Inorg. Chem., 1999, 38, 2734-2741

${ }^{118}$ L. Yang, D. R. Powell, R. P. Houser, Dalton Trans., 2007, 955-964

${ }^{119}$ R. D. Cortright; R. R. Davda; J. A. Dumesic, Nature 2002, 418, 964-967.

${ }^{120}$ D. A. J. Rand, R. M. Dell, Hydrogen Energy: Challenges and Prospects, RSCpublishing, 2007

${ }^{121}$ R. M. Navarro, M. A. Penã, J. L. G. Fierro, Chem. Rev. 2007, 107, 3952-3991

${ }^{122}$ U. Eberle, B. Müller, R. V. Helmolt, Energy Environ. Sci. 2012, 5, 8780-8798

${ }^{123}$ Fuel Cells Bull. 2018, 8, 6-7

${ }^{124}$ T. R. Burton, S. Surmeli, Nat. Law Rev. 2018, November 5.

125 J. Eppinger, K. W. Huang, ACS Energy Lett. 2017, 2, 188-195

${ }^{126}$ K. Sordakis, C. Tang, L. K. Vogt, H. Junge, . P. J. Dyson, M. Beller, G. Laurenczy, Chem. Rev. 2017, 118, 372-433

${ }^{127}$ E. A. Bielinski, P. O. Lagaditis, Y. Zhang, B. Q. Mercado, C. Würtele, W. H. Bernskoetter, N. Hazari, S. Schneider, J. Am. Chem. Soc. 2014, 136, 10234-10237

${ }^{128}$ R. Langer, Y. Diskin-Posner, G. Leitus, L. J. W. Shimon, Y. Ben-David, D. Milstein, Angew. Chem. Int. Ed. 2011, 50, 9948-9952

${ }^{129}$ F. Bertini, M. Glatz, N. Gorgas, B. Stoger, M. Peruzzini, L. F. Veiros, K. Kirchner, L.

Gonsalvi, Chem. Sci. 2017, 8, 5024-5029

${ }^{130}$ F. Bertini, N. Gorgas, B. Stöger, M. Peruzzini, L. F. Veiros, K. Kirchner, L. Gonsalvi, ACS Catal. 2016, 6, 2889-2893

${ }^{131}$ K. M. Waldie, A. L. Ostericher, M. H. Reineke, A. F. Sasayama, C. P. Kubiak, ACS Catal $2018,8,1313-1324$ 
132 J. Eppinger, K. W. Huang, ACS Energy Lett. 2017, 2, 188-195

${ }^{133}$ S. Kosourov, M. Jokel, E. M. Aro, Y. Allahverdiyeva, Energy. Environ. Sci. 2018, 11, 1431 1436

${ }^{134}$ M. Stephenson, L. H. Stickland, Biochem. J. 1931, 25, 205-214.

${ }^{135}$ P. M. Vignais, B. Billoud, Chem. Rev. 2007, 107, 4206-4272

${ }^{136}$ C. Greening, A. Biswas, C. R. Carere, C. J. Jackson, M. C. Taylor, M. B. Stott, G. M. Cook, S. E. Morales, Int. Soc. Micro. Eco. 2016, 10, 761-777

${ }^{137}$ W. Lubitz, B. Tumas, Chem. Rev. 2007, 107, 3900-3903

${ }^{138}$ A. Volbeda, M. H. Charon, C. Piras, E. C. Hatchikiran, M. Frey, J. C. Fontecilla-Camps, Nature, 1995, 373, 580-587

${ }^{139}$ W. Lubitz, E. J. Reijerse, J. Messinger, Energy. Env. Sci. 2008, 1, 15-31.

${ }^{140}$ J. W. Slater, S. C. Marguet, H. A. Monaco, H. S. Shafaat, J. Am. Chem. Soc. 2018, 140, 10250-10262

${ }^{141}$ D. Schilter, J. M. Camara, M. T. Huynh, S. Hammes-Schiffer, T. B. Rauchfuss, Chem. Rev. 2016, 116, 8693-8749

${ }^{142}$ A. D. Wilson, R. H. Newell, M. J. McNevin, J. T. Muckerman, M. R. Dubois, D. L. Dubois, J. Am Chem. Soc. 2006, 128, 358-366.

${ }^{143}$ J. Y. Yang, R. M. Bullock, W. J. Shaw, B. Twamley, K. Fraze, M. R. DuBois, D. L. DuBois, J. Am. Chem. Soc. 2009, 131, 5935-5945.

${ }^{144}$ R. Tatematsu; T. Inomata; T. Ozawa; H. Masuda, Angew. Chem. Int. Ed. 2016, 55, 1-5.

${ }^{145}$ L. Gan, T. L. Groy, P. Tarakeshwar, S. K. S. Mazinani, J. Shearer, V. Mujica, A. K. A. Jones, J. Am. Chem. Soc. 2015, 137, 1109-1115.

${ }^{146}$ H. Ogata, T. Krämer, H. Wang, D. Schilter, V. Pelmenschikov, M. Gastel, F. Neese, T. B. Rauchfuss, L. B. Gee, A. D. Scott, Y. Yoda, Y. Tanaka, W. Lubitz, S. P. Cramer, Nature Comm. 2015, https://doi.org/10.1038/ncomms8890

${ }^{147}$ B. E. Barton, C. M. Whaley, T. B. Rauchfuss, D. L. Gray, J. Am. Chem. Soc. 2009, 131, $6942-$ 6943.

${ }^{148}$ B. E. Barton, T. B. Rauchfuss, J. Am. Chem. Soc. 2010, 132, 14877-14885

${ }^{149}$ S. Ogo1, K. Ichikawa, T. Kishima, T. Matsumoto, H. Nakai, K. Kusaka, T. Ohhara, Science 2013, 339, 682-684

${ }^{150}$ D. Brazzolotto, M. Gennari, N. Queyriaux, T. R. Simmons, J. Pécaut, S. Demeshko, F. Meyer, M. Orio, V. Artero, C. Duboc, Nat. Chem. 2016, 8, 1054-1060.

${ }^{151}$ N. A. Eberhardt, H. Guan, Chem. Rev. 2016, 116, 8373-8426

${ }^{152}$ B. Desguin, T. Zhang, P. Soumillion, P. Hols, J. Hu, R. P. Hausinger, Science 2015, 349, 6669

${ }^{153}$ T. Xua, M. D. Wodricha, R. Scopellitia, C. Corminboeuf, X. Hua, PNAS 2017, 114, $1242-$ 1245

${ }^{154}$ D. H. Manz, P. C. Duan, S. Dechert, S. Demeshko, R. Oswald, M. John, R. A. Mata, F. Meyer J. Am. Chem. Soc. 2017, 139, 16720-16731

${ }^{155}$ P. C. Duan, D. H. Manz, S. Dechert, S. Demeshko, F. Meyer J. Am. Chem. Soc. 2018, 140, 4929-4939

${ }^{156}$ E. Ferretti, S. Dechert, S. Demeshko, M. C. Holthausen, F. Meyer, Angew. Chem. Int. Ed. 2019, 58, 1705-1709

${ }^{157}$ Manuscript in preparation

${ }^{158}$ J. I. Vlugt, E. A. Pidko, D. Vogt, M. Lutz, A. L. Spek, Inorg. Chem. 2009, 48, 7513-7515

${ }^{159}$ G. J. Kubas, Science 2006, 314, 1096-1097 
${ }^{160}$ T. He, N. P. Tsvetkov, J. G. Andino, X. Gao, B. C. Fullmer, K. G. Caulton, J. Am. Chem. Soc. 2010, 132, 910-911.

${ }^{161}$ S. Chen, R. Rousseau, S. Raugei, M. Dupuis, D. L. DuBois, R. M. Bullock, Organometallics 2011, 30, 6108-6118

${ }^{162}$ P. A. Dub, J. C. Gordon, Nature Rev. Chem. 2018, https://doi.org/10.1038/s41570-0180049-z

${ }^{163}$ W. H. Harman, J. C. Peters J. Am. Chem. Soc. 2012, 134, 5080-5082

${ }^{164}$ T. P. Lin, J. C. Peters, J. Am. Chem. Soc. 2014, 136, 13672-13683

${ }^{165}$ D. Manz, Ph.D. Thesis, University of Göttingen, 2016

${ }^{166}$ C. Yoo, Y. Lee, Angew. Chem. Int. Ed. 2017, 56, 9502-9506

${ }^{167}$ F. Speiser, P. Braunstein, L. Saussine, Acc. Chem. Res. 2005, 38, 784-793

${ }^{168}$ K. V. Vasudevan, B. L. Scott, S. K. Hanson, Eur. J. Inorg. Chem. 2012, 4898-4906

${ }^{169}$ P. L. Theofanis, W. A. Goddard, Organometallics 2011, 30, 4941-4948

${ }^{170}$ D. L. Thorn, R. Hoffmann, J. Am. Chem. Soc. 1978,100, 2079-2090

${ }^{171}$ E. F. Lutz, J. Chem. Edu. 1986, 63, 605-607

172 J. Echeverria, Cryst. Growth Des. 2017, 17, 2097-2103.

${ }^{173}$ I. Alkorta, I. Rozas, J. Elguero, Chem Soc. Rev. 1998, 27, 163-170.

${ }^{174}$ R. H. Crabtree, O. Eisenstein, G. Sini, E. Peris, J. Organo. chem. 1998, 576, 7-11.

${ }^{175}$ Q. Liu, R. Hoffmann, J. Am. Chem. Soc. 1995, 117, 10108-10112.

${ }^{176}$ T. B. Richardson, S. Gala, R. H. Crabtree, J. Am. Chem. Soc. 1995, 117, 12875-12876.

${ }^{177}$ R. H. Crabtree, P. E. M. Siegbahn, O. Eisenstein, A. L. Rheingold, T. F. Koetzle Acc. Chem. Res. 1996, 29, 348-354

${ }^{178}$ R. Custelcean, J. E. Jackson, Chem. Rev. 2001, 101, 1963-1980.

${ }^{179}$ S. J. Grabowski, W. A. Sokalski, J. Leszczynski, J. Phys. Chem. A 2005, 109, 4331-4341.

${ }^{180}$ I. Alkorta, J. Elguero, S. J. Grabowski, J. Phys. Chem. A 2008, 112, 2721-2727.

${ }^{181}$ N. V. Belkova, L. M. Epstein, O. A. Filippov, E. S. Shubina, Chem. Rev. 2016, 116, 8545-8587.

${ }^{182}$ D. Milstein, J. C. Calabrese, I. D. Williams, J. Am. Chem. Soc. 1986, 108, 6387-6389.

${ }^{183}$ R. C. Stevens, R. Bau, D. Milstein, O. Blum, T. Koetzle, J. Chem. Soc. Dalton Trans., 1990,0, 1429-1432.

${ }^{184}$ A. J. Edwards, S. Elipe, M. A. Esteruelas, F. J. Lahoz, L. A. Oro, C. Valero, Organometallics 1997, 16, 3828-3836.

${ }^{185}$ L. S. Van Der Stays, J. Eckert, O. Eisenstein, J. H. Hall, J. C. Huffman, S. A. Jackson, T. F. Koetzle, G. J. Kubas, P. J. Vergamini, K. G. Caulton, J. Am. Chem. Soc. 1990, 112, 4831-4841.

${ }^{186}$ M. L. Buil, M. A. Esteruelas, E. Oñate, N. Ruiz, Organometallics 1998, 17, 3346-3355.

${ }^{187}$ A. J. Lough, S. Park, R. Ramachandran, R. H. Morris, J. Am. Chem. Soc. 1994, 116, 83568357.

188 J. A. Ayllon, C. Gervaux, S. Sabo-Etienne, B. Chaudret, Organometallics 1997, 16, 2000-2002.

${ }^{189}$ H. S. Chu, C. P. Lau, K. Y. Wong, W. T. Wong, Organometallics 1998,17, 2768-2777.

${ }^{190}$ W. K. Fung, X. Huang, M. L. Man, S. M. Ng, M. Y. Hung, Z. Lin, C. P. Lau, J. Am. Chem. Soc. 2003, 125, 11539-11544.

${ }^{191}$ P. A. Dub, N. J. Henson, R. L. Martin, J. C. Gordon, J. Am. Chem. Soc. 2014, 136, 3505-3521.

192 S. J. Robinson Connelly, D. M. Heinekey, Chem. Commun. 2017, 53, 669-676.

${ }^{193}$ R. M. Bullock, A. M. Appel, M. L. Helm, Chem. Commun. 2014, 50, 3125-3143.

${ }^{194}$ E. B. Hulley, M. L. Helm, R. M. Bullock, Chem. Sci. 2014, 5, 4729-4741. 
195 T. Liu, X. Wang, C. Hoffmann, D. L. DuBois, R. M. Bullock, Angew. Chem. Int. Ed. 2014, 53, $5300-5304$.

${ }^{196}$ T. Liu, M. R. DuBois, D. L. DuBois, R. M. Bullock, Energy. Env. Sci. 2014, 7, 3630-3639.

${ }^{197}$ S. Zhang, A. M. Appel, R. M. Bullock, J. Am. Chem. Soc. 2017, 139, 7376-7387.

${ }^{198}$ H. Li, M. B. Hall, ACS Catal. 2015, 5, 1895-1913

${ }^{199}$ A. Friedrich, M. Drees, J. Schmedt auf der Günne, S. Schneider, J. Am Chem. Soc. 2009, 131, $17552-17553$.

${ }^{200}$ G. J. Kubas, Chem. Rev. 2007, 107, 4152-4205.

${ }^{201}$ R. H. Morris, Chem. Rev. 2016, 116, 8588-8654.

${ }^{202}$ R. H. Crabtree, Chem. Rev. 2016, 116, 8750-8769.

${ }^{203}$ M. A. Esteruelas, A. M. López, M. Oliván, Chem. Rev. 2016, 116, 8770-8847.

${ }^{204}$ S. J. Connelly, A. C. Zimmerman, W. Kaminsky, D. M. Heinekey, Chem. Eur. J. 2012, 18, $15932-15934$.

${ }^{205}$ W. H. Harman, T. P. Lin, J. C. Peters, Angew. Chem. Int. Ed. 2014, 53, 1081-1086.

${ }^{206}$ C. Tsay; J. C. Peters, Chem. Sci., 2012, 3, 1313-1318.

${ }^{207}$ B. J. Boro, E. N. Duesler, K. I. Goldberg, R. A. Kemp, Inorg. Chem. 2009, 48, 5081-5087.

${ }^{208}$ V. A. Levina, A. Rossin, N. V. Belkova, M. R. Chierotti, L. M. Epstein, O. A. Filippov, R. Gobetto, L. Gonsalvi, A. Llledòs, E. S. Shubina, F. Zanobini, M. Peruzzini, Angew. Chem. Int. Ed. 2011, 123, 1403-1406.

${ }^{209}$ Z. Yu, J. M. Wittbrodt, A. Xia, M. J. Heeg, H. B. Schlegel, C. H. Winter; Organometallics 2001,17, 4301-4303.

${ }^{210}$ P. Duan, Ph.D. Thesis, University of Göttingen, 2018

${ }^{211}$ V. I. Bakhmutov, Practical NMR Relxation for Chemists, John Wiley $\mathcal{E}$ Sons, 2004, 8, 108

${ }^{212}$ T. A. Luther, D. M. Heinekey, Inorg. Chem. 1998, 37, 127-132

${ }^{213}$ P. Goursot, A. Römer, J. Ustenko, S. Samanta, M. John, S. Dechert, R. A. Mata, F. Meyer, Manuscript in preparation.

${ }^{214}$ B.B Corson, E. Depp, G. Black, J. Org. Chem. 1949, 14, 14-21. 


\section{List of Abreviations}

\begin{tabular}{ll}
$\AA$ & Angström $(0.1$ nanometer $)$ \\
acac & acetylacetonato \\
{$\left[\mathrm{BAr}_{4}{ }_{4}\right]^{-}$} & {$\left[\left(3,5-\left(\mathrm{CF}_{3}\right)_{2} \mathrm{C}_{6} \mathrm{H}_{3}\right)_{4} \mathrm{~B}\right]^{-}$} \\
atm & atmosphere \\
d & chemical shift $(\mathrm{NMR})$ \\
DHB & dihydrogen bond \\
d & doublet (NMR) \\
EA & elemental analysis \\
$\varepsilon$ & extinction coefficient \\
eq & equivalent \\
ESI & electron spray ionisation \\
h & hour \\
hs & high spin \\
IR & infrared \\
$J$ & NMR coupling constant \\
KOBu & potassium tert-butoxide \\
ls & low spin \\
min & minute \\
m & multiplet (NMR) \\
NMR & nuclear magnetic resonance \\
NOESY & nuclear Overhauser and exchange spectroscopy \\
ppm & parts per million \\
RT & room temperature \\
s & singlet (NMR) \\
THF & tetrahydrofuran \\
{$[2,2,2]$} & Cryptand 222 or 4,7,13,16,21,24-hexaoxa-1,10- \\
& diazabicyclo[8.8.8]hexacosane \\
\hline &
\end{tabular}




\title{
List of scientific contributions
}

\author{
Manuscripts in preparation
}

P. Goursot, A. Römer, J. Ustenko, S. Samanta, M. John, S. Dechert, R. A. Mata, F. Meyer, Intramolecular frustrated dihydrogen bond in a Two-In-One pincer dinickel complex and water assisted reversible splitting of $\mathrm{H}_{2}$.

A. Gers-Barlag, P. Goursot, S. Dechert, F. Meyer, Sequential double dearomatization at a Rh(I) dinuclear complex.

\section{Presentation at conferences}

P. Goursot, "Activation of $\mathrm{H}_{2} \mathrm{O}$ by metal-ligand cooperation. A Ni-O-H ${ }^{\delta^{+}} \ldots . . \delta^{-} \mathrm{H}-\mathrm{Ni}$ motif that allows $\mathrm{H}_{2}$ Splitting”, Göttinger Chemie-Forum, Göttingen, Germany, 31 May 2018.

P. Goursot, "Mechanistic investigation of cooperative $\mathrm{H}_{2}$ activation by dinuclear nickel complexes of the "Two-In-One" pincer scaffold", ICOMC 2018, Florence, Italy, 15-20 July 2018.

\section{Posters}

P. Goursot, S. Samanta, S. Demeshko, S. Dechert, F. Meyer, "Cooperative substrate activation by dinuclear nickel complexes of the "Two-In-One" pincer scaffold", Göttinger Chemie-Forum, Göttingen, Germany, 22 June 2017.

P. Goursot, S. Samanta, S. Demeshko, S. Dechert, F. Meyer, "Cooperative substrate activation by dinuclear nickel complexes of the "Two-In-One" pincer scaffold", EICC-4, Copenhagen, Denmark, 2-5 July 2017.

P. Goursot, S. Samanta, S. Demeshko, S. Dechert, F. Meyer, "Cooperative substrate activation by dinuclear nickel complexes of the "Two-In-One" pincer scaffold", AgiChem, Göttingen, Germany, 6-9 August 2017.

P. Goursot, S. Samanta, S. Demeshko, S. Dechert, F. Meyer, "Cooperative Substrate Activation by Dinuclear Nickel Complexes of the "Two-In-One” Pincer Scaffold”, ICASEC, Göttingen, Germany, 24-29 September 2017.

P. Goursot, A. Römer, J. Ustenko, S. Samanta, M. John, S. Dechert, R. A. Mata, F. Meyer, "Mechanistic investigation of cooperative $\mathrm{H}_{2}$ activation by dinuclear nickel complexes of the “Two-In-One” pincer scaffold”, ICOMC 2018, Florence, Italy, 15-20 July 2018.

P. Goursot, A. Römer, J. Ustenko, S. Samanta, M. John, S. Dechert, R. A. Mata, F. Meyer, "Mechanistic investigation of cooperative $\mathrm{H}_{2}$ activation by dinuclear nickel complexes of the “Two-In-One” pincer scaffold”, NiKas 2018, Göttingen, Germany, 19-20 September 2018. 
Summerschool

ICASEC, Göttingen, Germany, 24-29 September 2017. 